An Analysis of Methods and

Models for Assessing the

Direct and Indirect Economic

Impacts of $\mathrm{CO}_{2}$-Induced

Environmental Changes in the

Agricultural Sector of the

U.S. Economy

\title{
August 1982
}

Prepared for the U.S. Department of Energy under Contract DE-AC06-76RLO 1830

Pacific Northwest Laboratory Operated for the U.S. Department of Energy by Battelle Memorial Institute 


\title{
DISCLAIMER
}

This report was prepared as an account of work sponsored by an agency of the United States Government. Neither the United States Government nor any agency thereof, nor any of their employees, makes any warranty, express or implied, or assumes any legal liability or responsibility for the accuracy, completeness, or usefulness of any information, apparatus, product, or process disclosed, or represents that its use would not infringe privately owned rights. Reference herein to any specific commercial product, process, or service by trade name, trademark, manufacturer, or otherwise, does not necessarily constitute or imply its endorsement, recommeridation, or favoring by the United States Government or any agency thereof. The views and opinions of authors expressed herein do not necessarily state or reflect those of the United States Government or any agency thereof.

\author{
PACIFIC NORTHWEST L.ABORATORY \\ operated by \\ BATTELLE \\ for the \\ UNITED STATES DEPARTMENT OF ENERGY \\ under Contract DE-AC06-76RLO 1830
}

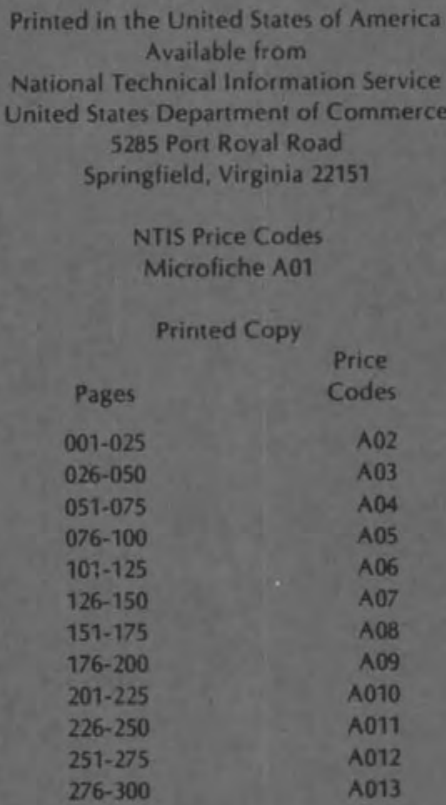


AN ANALYSIS OF METHODS AND MODELS FOR ASSESSING THE DIRECT AND INDIRECT ECONOMIC IMPACTS OF $\mathrm{CO}_{2}-$ INDUCED ENV IRONMENTAL CHANGES IN THE AGRICULTURAL SECTOR OF THE U.S. ECONOMY
J. M. Callaway
F. J. Cronin
J. W. Currie
J. Tawil

August 1982

Prepared for the U.S. Department of Energy under Contract DE-ACO6-76RLO 1830

Pac if ic Northwest Laboratory Richland, Washington 99352 
. 


\section{SUMMARY}

This research was conducted by the Pacific Northwest Laboratory (PNL) for the Division of Carbon Dioxide Research within the Office of Basic Energy Sciences, U.S. Department of Energy (DOE). The overall purpose of th is research was to assist DOE in developing methods for assessing the direct and indirect economic impacts due to the effects of increases in the ambient concentration of $\mathrm{CO}_{2}$ on agricultural production. Specifically, the primary objectives were:

- To determine what economic methods and models have been developed or need to be developed to assess the direct and indirect economic impacts of $\mathrm{CO}_{2}$-induced environmental changes in the agricultural sector of the U.S. economy.

- To provide information and recommendations which will enhance DOE's efforts to assess the direct and indirect economic impacts associated with $\mathrm{CO}_{2}$ buildup, not only as they relate to the agricultural sector, but to other sectors of the U.S. economy as wel1.

Several tasks were required to accomplish these objectives. First, a comprehensive literature search was undertaken to determine what types of models and methods have been developed, which could be effectively used to conduct assessments of the direct and indirect economic impacts of $\mathrm{CO}_{2}$ buildup. Specific attention was focused upon: (1) mode is and methods for assessing the physical impacts of $\mathrm{CO}_{2}$-induced environmental changes on crop yields, (2) national and multi-regional agricultural sector models, and (3) macroeconomic models of the U.S. economy. The second task of this research involved a thorough investigation of the research efforts being conducted by other public and private sector organizations in order to determine how more recent analytical methods being developed outside of DOE could be effectively integrated into a more comprehensive analys is of the direct economic impacts of $\mathrm{CO}_{2}$ buildup. The third and final task of this research involved 
synthesizing the information gathered in the first two tasks into a systematic framework for assessing the direct and indirect economic impacts of $\mathrm{CO}_{2}$-induced environmental changes originating in the agricultural sector of the U.S. economy.

The major findings of this research are listed below.

- It is anticipated that the direct economic impacts of $\mathrm{CO}_{2}$ on the agricultural sector and the indirect economic impacts caused by spillover effects from agriculture to other sectors of the economy will be pervasive; however, the direction and magnitude of these impacts on producers and consumers cannot be determined a prior $i$.

- Existing agricultural sector models can be effectively linked to macroeconomic models of the U.S. economy to assess the potential scope and magnitude of the economic impacts associated with $\mathrm{CO}_{2}$-induced environmental changes that originate in the agricultural sector.

- Because of the time frame involved, it is highly unlikely that any type of economic analys is will be able to predict these impacts with any reliable degree of confidence at this time; however, existing models and methods can be used at this time to assess the sensitivity of the economy to both sudden and gradual changes in the ambient concentration of $\mathrm{CO}_{2}$. This type of analys is is important because it will point out potential trouble spots in the agricultural sector and elsewhere in the economy which can then be assessed more closely using subjective judgments about the potential of the economy to adjust to these problems in the long-run. 


\section{CONTENTS}

SUMMAR

1.0 INTRODUCTION

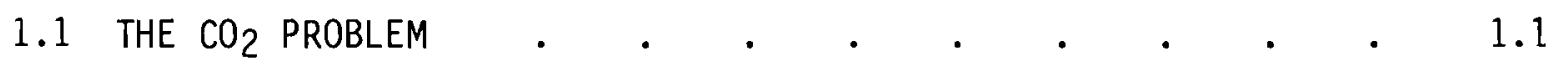

1.2 LEGISLATIVE AND ADMINISTRATIVE BACKGROUND $\quad$ • $\quad$ • $\quad$ • $\quad 1.2$

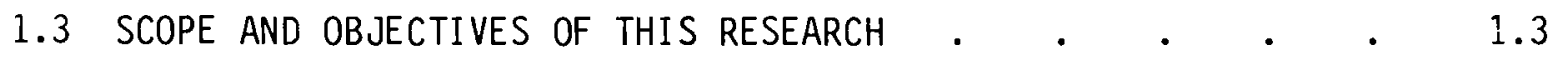

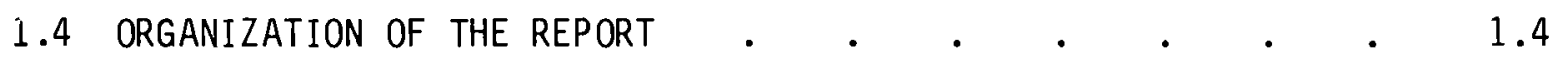

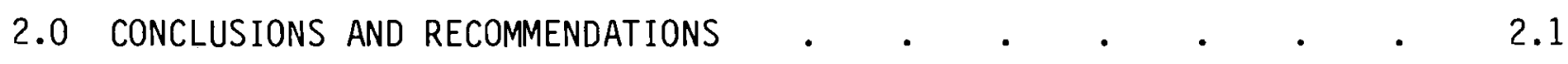

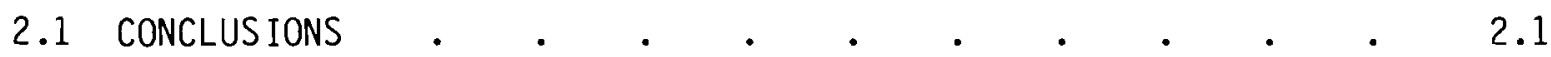

2.1.1 Identification of Physical Impacts of $\mathrm{CO}_{2}$
Buildup on Crop Yields.

2.1.2 Identification of Economic Potential Impacts Due to $\mathrm{CO}_{2}$-Induced Changes in the Environment $. \quad . \quad . \quad 2.2$

2.1.3 Methods for Assessing the Impacts of $\mathrm{CO}_{2}$ on Climate $. \quad . \quad . \quad . \quad . \quad . \quad . \quad . \quad . \quad 2.6$

2.1.4 Methods for Assessing the Impacts of $\mathrm{CO}_{2}-$ Induced Environmental Changes on Crop

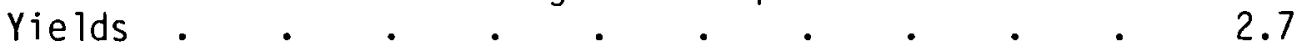

2.1.5 Methods for Assessing the Direct Economic Impacts of $\mathrm{CO}_{2}$-Induced Changes in the

Environment . $. \quad . \quad . \quad . \quad . \quad . \quad . \quad . \quad 2.10$

2.1.6 Methods for Assessing the Indirect Economic

Impacts of $\mathrm{CO}_{2}$-Induced Changes in the

Environment . . . . . . . . . . . 2.13

2.1.7 Strategies for Assessing the Economic Impacts
of $\mathrm{CO}_{2}$-Induced Changes in the Environment $\quad . \quad 2.16$

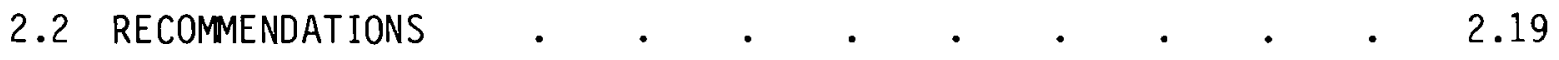

2.2.1 Research Scope . . . . . . . . . . 2.19 
2.2.2 Development of Climate Models . . . . . 2.20

2.2.3 Selection of Methods for Assessing the Impacts

of $\mathrm{CO}_{2}$-Induced Environmental Changes on

Crop Yields . . . . . . . . . . . . 2.20

2.2.4 Selection of Economic Models for Conducting
Impact Assessments $. \quad . \quad . \quad . \quad . \quad 2.21$

2.2.5 Modifications to Existing Models . . . . . 2.22

3.0 $\mathrm{CO}_{2}$ AND THE ECONOMICS OF ENVIRONMENTAL CHANGE IN

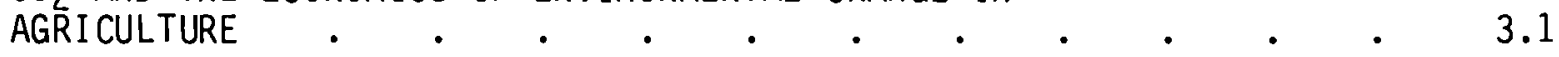

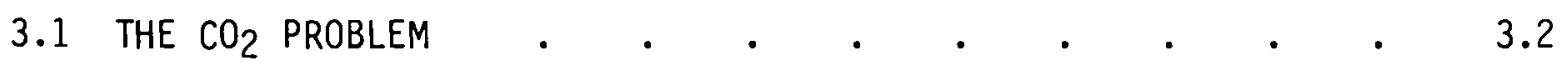

3.2 ENVIRONMENTAL EFFECTS OF $\mathrm{CO}_{2}$ ON CROP YIELDS $\quad . \quad$ • $\quad 3.6$

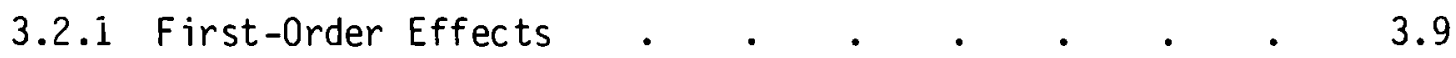

3.2.2 Second-Order Effects . $\quad . \quad$. $\quad . \quad$. $\quad . \quad 3.14$

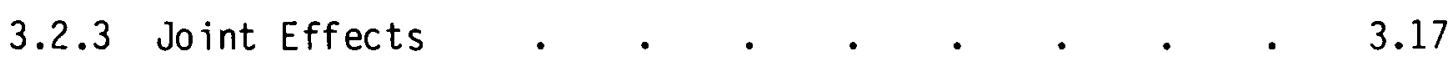

3.3 DIRECT ECONOMIC IMPACTS OF $\mathrm{CO}_{2}$-INDUCED ENVIRONMENTAL

3.3.1 Resource Allocation by the Firm . . . . . 3.20

3.3.2 Market Supp Ty and Demand . . . . . . . 3.31

\begin{tabular}{l}
3.4 INDIRECT IMPACTS OF $\mathrm{CO}_{2}$-INDUCED ENVIRONMENTAL \\
CHANGES \\
\hline
\end{tabular}

3.4.1 Indirect Impacts on Factor Markets . . . . . 3.43

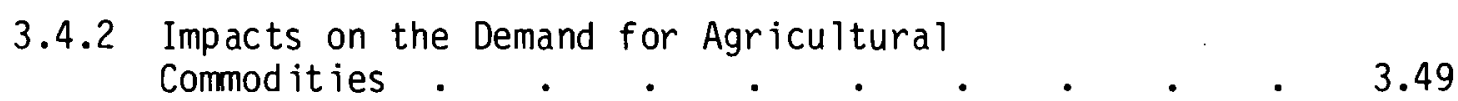

3.5 SUMMARY OF DIRECT AND INDIRECT ECONOMIC IMPACTS $\quad . \quad$ : $\quad 3.54$

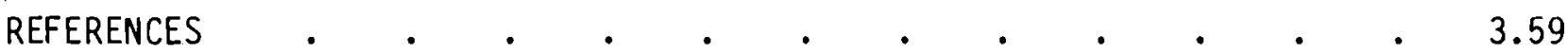

\begin{tabular}{l}
4.0 VALUATION OF INFORMATION RELATED TO UNCERTAIN ENVIRONMENTAL \\
CHANGES \\
\hline
\end{tabular} 
4.1 RESPONSES OF AGRICULTURAL FIRMS TO ENVIRONMENTAL

UNCERTAINTY $\cdot \quad \cdot \quad \cdot \quad \cdot \quad \cdot \quad \cdot \quad \cdot \quad \cdot \quad \cdot \quad \cdot 4.3$

4.1 .1 Assumptions . . . . . . . . . . . . 4.4

4.1.2 Behavior of the Firm in the Face of Environmental
Uncertainty. . . . . . . . . . 4.5

4.2 THE DEMAND FOR CLIMATE RELATEd INFORMATION • • • • • 4.17

4.2.1 Bayesian Approach . . . . . . . . 4.18

4.2.2 Risk Aversion and the Value of Information. . . 4.27

4.2.3 Market Failure and the Value of Information . 4.31

REFERENCES

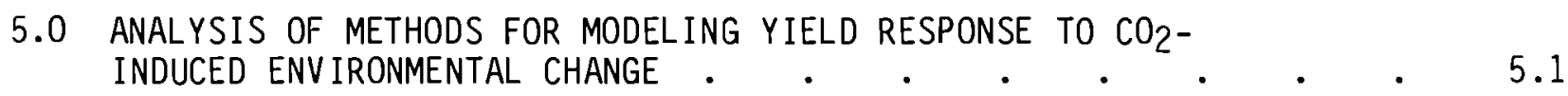

5.1 METHODOLOGICAL ISSUES IN FORECASTING $\mathrm{CO}_{2}$-INDUCED

CROP YIELD CHANGES $\cdot$.

5.1.1 Climate Forecasts and Yield Prediction . $\quad$. 5.2

5.1.2 The Independence of Environmental Effects on
Crop Production . . . . . . . . . 5.8

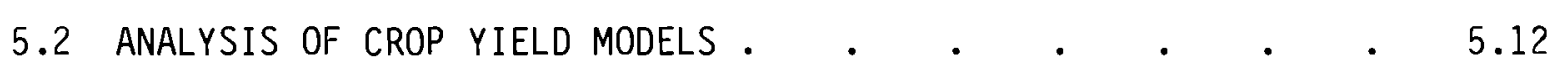

5.2.1 Panel Survey Approach . . . . . . . 5.13

5.2 .2 Statistical Yield Models . . . . . . 5.20

5.2 .3 Simulation Models . $. \quad . \quad . \quad . \quad . \quad . \quad 5.35$

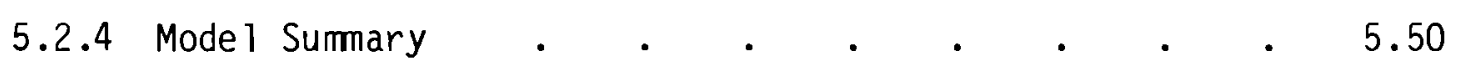

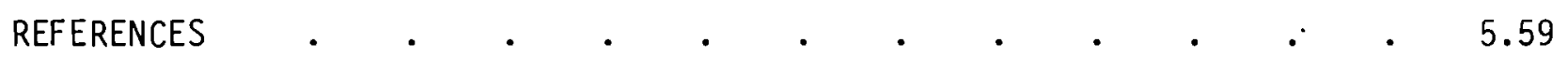

6.0 ANALYSIS OF METHODS AND MODELS FOR ASSESSING THE DIRECT

ECONOMIC IMPACTS OF $\mathrm{CO}_{2}$-INDUCED ENVIRONMENTAL CHANGES

ON THE AGRICULTURAL SECTOR OF THE U.S. ECONOMY

6.1 METHODOLOGICAL ISSUES IN ASSESSING THE DIRECT ECONOMIC
IMPACTS OF $\mathrm{CO}_{2}$-INDUCED ENVIRONMENTAL CHANGES.$\cdot$.
. 
6.1.1 Alternative Strategies for Direct Impact

6.1.2 Dynamic Adjustments in Economic Ana lysis . . 6.10

6.1.3 Modeling the Impact of Climate Variability on the Agricultural Sector . . . . . . 6.11

6.1.4 Regional and Commodity Disaggregation in Agricultural Sector Models . . . . . . 6.16

6.1.5 Linkages to the Rest of the World . . . . 6.19

6.1.6 Data Needs and Availability. . . . . . 6.23

6.1.7 Statistical Accuracy and Stability of Agricultural Sector Models . . . . . . 6.24

6.2 ANALYSIS OF AGRICULTURAL SECTOR MODELS . . . . . . 6.26

6.2.1 Econometric Models of the Agricultural Sector . . 6.28

6.2.2 Normative Models of the U.S. Agricultural Sector. . . . . . . . . . 6.46

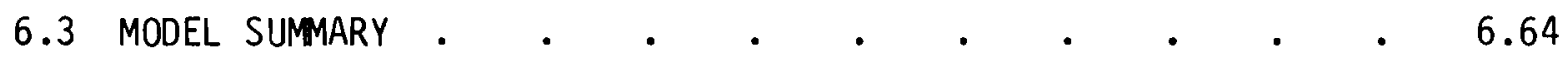

6.3.1 Research Strategies . . . . . . . 6.65

6.3 .2 Scope of Impacts . . . . . . . . . 6.67

6.3 .3 Recommendations . . . . . . . . 6.70

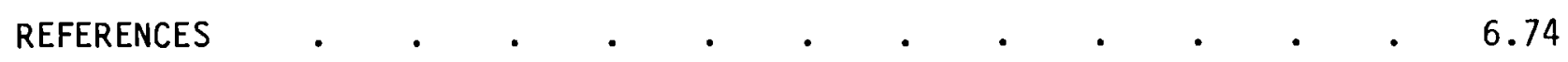

7.0 ANALYSIS OF METHODS AND MODELS FOR ASSESSING THE INDIRECT

IMPACTS OF $\mathrm{CO}_{2}$-INDUCED ENVIRONMENTAL CHANGES ON OTHER

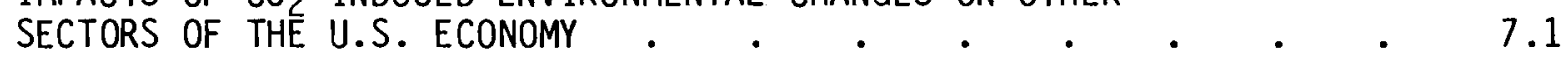

\begin{tabular}{l}
7.1 ANALYSIS OF ALTERNATIVE METHODS FOR MODELING THE \\
NATIONAL ECONOMY \\
\hline
\end{tabular}

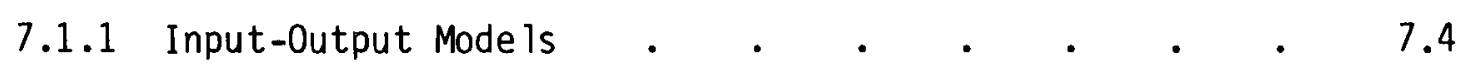

7.1.2 Input-Output Based Econometric Models . . . . 7.13 
7.1.3 Normative General Equilibrium Models • . . 7.22

7.1.4 Regional Econometric Models . . . . . . . 7.31

7.2 INDIRECT IMPACTS ON THE ALLOCATION OF PRIMARY

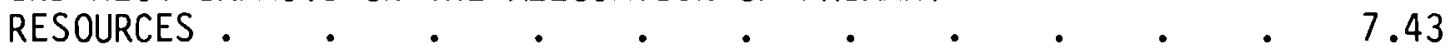

7.2.1 Resource Based Input-Output Models . . . . 7.44

7.2.2 Normative Models . . . . . . . . . 7.52

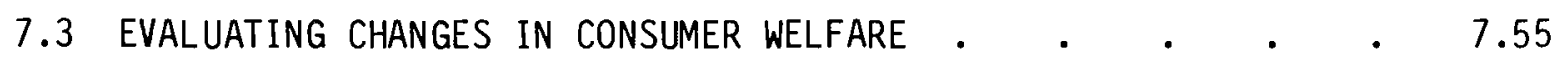

7.3.1 Measures of Consumer Welfare . . . . . $\quad$. 7.56

7.3.2 Estimating Changes in Consumer Welfare $. \quad . \quad . \quad 7.59$

7.3.3 Applied Analys is of a Consumer Welfare Changes . . 7.64

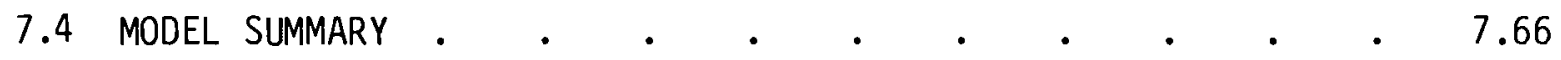

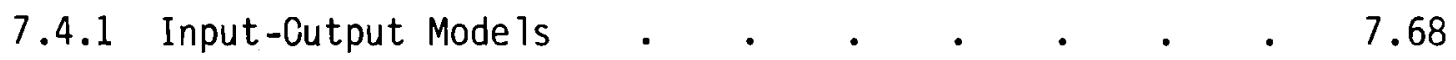

7.4.2 Input-Output Based Econometric Models . $\quad$. 7.70

7.4.3 Normative General Equilibrium Models . . . . 7.70

7.4.4 Multi-Regional Econometric Models . $\quad$. $\quad$. $\quad 7.71$

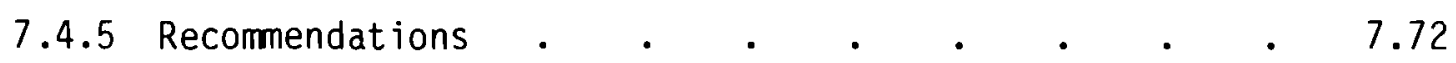

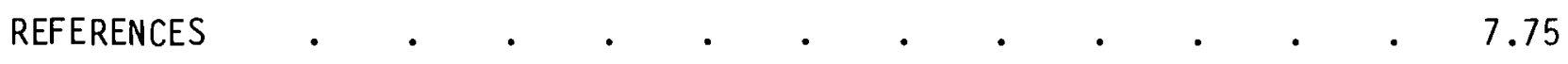




\section{FIGURES}

3.1 Path Diagram of Environment-Crop Interactions $. \quad . \quad$. $\quad 3.8$

3.2 The Firm's Demand for a Variable Input . . . . . . . 3.27

3.3 The Determination of the Optimal Output of the Firm . . . 3.29

3.4 Determination of Market Price and Quantity Equilibrium . . . $\quad 3.34$

3.5 Determination of Input Price and Quantity Equilibrium for 3.46

4.1 Flow Diagram of Decision-Making Process Under Uncertainty $\quad$ • $\quad 4.7$

4.2 Relationship Between Cost and Time Remaining to Complete

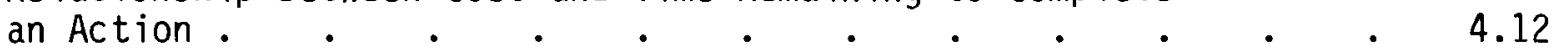

5.1 Schematic Diagram of the Climate Response Model . $\quad$ • . $\quad$. 5.16

5.2 A Systems Dynamics Conceptualization of the Nitrogen and Carbyohydrate Materials Balance in Cotton $. \quad . \quad . \quad$. 5.37

6.1 Hypothetical Effect of $\mathrm{CO}_{2}$ on International Trade $\quad . \quad$. $\quad$ - 6.21

6.2 A Schematic Diagram of the CARD Recursive (CR) Model . . . $\quad 6.53$

6.3 Illustration of Producer and Consumer Surplus . $\quad$. $\quad$. $\quad 6.56$

6.4 Linearization of Willingness to Pay Functions $\quad . \quad$ e $\quad$ • $\quad$ e $\quad 6.57$

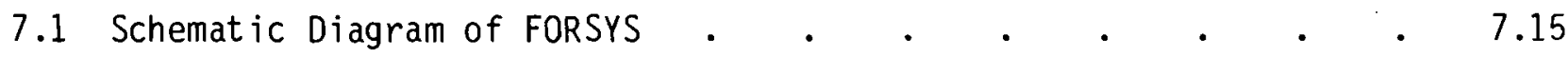

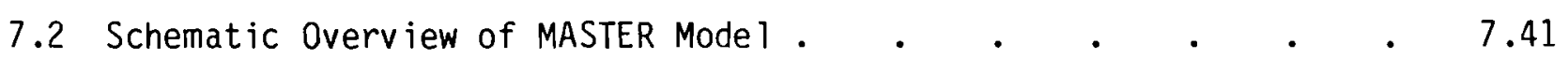




\section{TABLES}

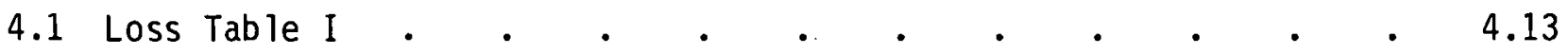

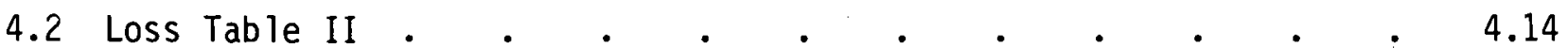

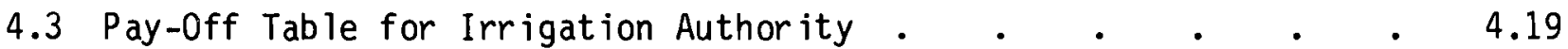

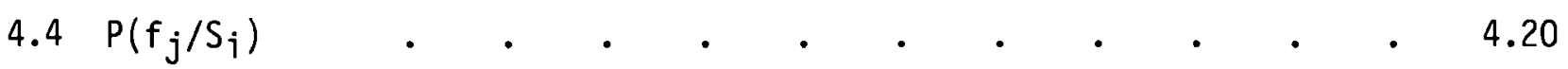

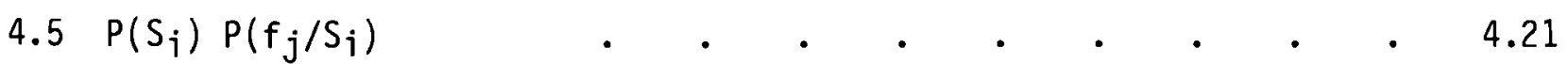

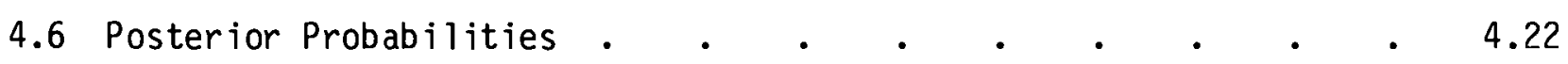

4.7 Expected Pay-Offs With and Without Climate Forecast . . . 4.22

5.1 Regression Coefficients for Multiple Regression Analys is $\quad 5.26$

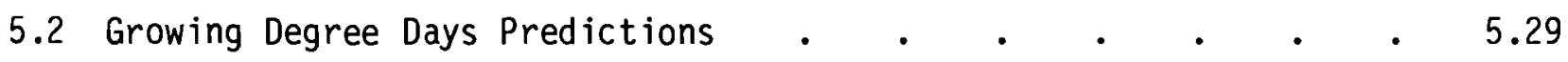

5.3 Some Currently Active Crop Modeling Efforts . . . . . $\quad$. 5.40

5.4 Sumary of Crop Yield Model Characteristics . $\quad$. $\quad$. $\quad$. $\quad 5.52$

5.5 Meteorological Data Mismatches Between General Circulation

6.i Agricultural Sector Models Reviewed for this Report . . . $\quad 6.29$

6.2 Summary of Agricultural Sector Model Characteristics . $\quad$ • $\quad 6.64$

6.3 Sumary of Structural Features and Recommended Changes to
AGGSIM and CARD Tatonnement Models . . . . . . . 6.72

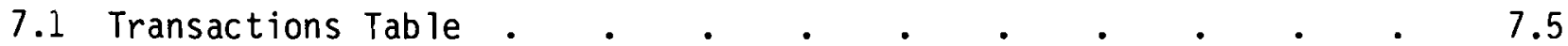

7.2 Total Demand and Supply Multipliers for Selected Industries

7.3 Summary of Structural Features of Models for Determining the
Ind irect Impacts of $\mathrm{CO}_{2}$-Induced Environmental Changes

7.4 Summary of Selected Structural Features of FORSYS, MASTER and the Proposed Normative General Equilibrium Model (NGE), Including Recommended Changes to FORSY . . . . . . 7.73 



\subsection{INTRODUCTION}

This research was performed by the P.acific Northwest Laboratory (PNL) for the Division of Carbon Dioxide Research within the Office of Basic Energy Sciences of the U.S. Department of Energy (DOE). It was undertaken primarily to assist DOE in developing a capability to assess the economic impacts associated with the buildup of $\mathrm{CO}_{2}$ in the earth's atmosphere.

\section{$1.1 \quad$ THE $\mathrm{CO}_{2}$ PROBLEM}

It has been estimated that, prior to the industrial revolution, the concentration of $\mathrm{CO}_{2}$ in the earth's atmosphere was about 290 parts per million by volume (ppmv). By 1980, the ambient concentration of $\mathrm{CO}_{2}$ had risen to around $338 \mathrm{ppmv}$ and is currently increasing at a rate of about $1.5 \mathrm{ppmv} / \mathrm{yr}$. While there is still much to be learned about the global carbon cycle which regulates ambient $\mathrm{CO}_{2}$, there is almost universal agreement within the scientific community that the buildup of $\mathrm{CO}_{2}$ in the earth's atmosphere has largely been due to the burning of fossil fuels. Estimates of the future rate of growth of ambient $\mathrm{CO}_{2}$ vary widely; however, the broad consensus is that it will take around 50 to 100 years for the concentration of $\mathrm{CO}_{2}$ in the atmosphere to reach a level twice that of the pre-industrial period.

As that happens, there is wide agreement that the earth's surface will become gradually warmer due to the green-house effect and that temperature increases will generally be greater as one moves away from the equator. Beyond that, very little is known. Potential effects on climate in the 21 st century include the creation of drought-prone regions in North America and Central Asia, melting of the West Antarctic ice sheet with a subsequent rise in world-wide sea levels of about 5 meters, and the opening of the Northwest passage due to the melting of the Arctic Ocean. All of these events have the potential to impact heavily and unevenly on the social, economic and political structures of many different nations. 


\subsection{LEGISLATIVE AND ADMINISTRATIVE BACKGROUND}

Partly in response to concerns raised about these potential problems, Congress passed, and the President signed into law, P.L. 95-367, the National Climate Program Act. This Act established a national program designed to "assist the nation and the world to understand and respond to natural and man-induced climate processes and their implications." The first two program elements identified in the Act are:

- assessments of the effect of climate on the national environment, agricultural production, energy supply and demand, land and water resources, transportation, human health and national security;

- basic and applied research to improve the understanding of climate processes, national and man-induced, and the social, economic, and political implications of climate change.

In order to further these objectives, the Secretary of Commerce was charged with the responsibility for implementing a National Climate Program and coordinating the climate-related research of other executive departments and agencies. The Act also mandated the development of a 5-year plan as a basis for achieving the objectives set forth in the Act.

The National Climate Program Five-Year Plant (NOAA 1980) defines six "Principle Thrusts" to which the highest research priorities have been assigned. Research on carbon dioxide, the environment and society constitutes one of these principle thrusts, with the lead responsibility for conducting research in this area belonging to the DOE. Within that department, this function has been assigned to the Division of Carbon Dioxide Research in the Office of Basic Energy Sciences. The research reported in this document was undertaken as a part of this Division's Carbon Dioxide Research Program. It represents a first step in examining alternative methods for quantifying the economic impacts associated with future increases in the concentration of $\mathrm{CO}_{2}$ in the earth's atmosphere. 


\subsection{SCOPE AND OBJECTIVES OF THIS RESEARCH}

This report focuses on the development of methods for assessing the possible direct and indirect economic impacts of $\mathrm{CO}_{2}$-induced changes in the natural and physical environment which could affect the production of agricultural commodities. Its scope does not directly embrace the development of methods for examining the economic impacts of $\mathrm{CO}_{2}$ buildup that might originate in other sectors of the economy. This does not imply that the direct and indirect economic impacts which could originate in other sectors of the economy will be unimportant. Indeed, $\mathrm{CO}_{2}$-induced changes in the terrestrial biosphere have the potential to directly affect not only the technological relationships which govern the production of goods and services by a great many producing sectors within the economy, but also the preferences of consumers for different kinds of $c l$ imate and climate-related amenities. However, the most immediate impacts are likely to occur in those industries which are most closely tied through the natural and physical environments to atmospheric $\mathrm{CO}_{2}$, namely: the agricultural, forestries and fisheries sectors.

While the agricultural sector is by far the largesi of these three, it accounts for only about. $3 \%$ of total gross national product, annually. However, the importance of agriculture in assessing the economic impacts of $\mathrm{CO}_{2}$ buildup does not lie in its size, but rather in the great strategic importance of this sector in the U.S. and world food situation. Between 1970 and 1980, U.S. agricultural exports averaged about $22 \%$ of the total value of all goods and services exported from the U.S. The importance of the U.S. agricultural sector in world food markets is further illustrated by the fact that during this same period corn and soybean exports from the U.S. both constituted about 75\% of total exports in their respective world markets. The agricultural sector is also important because many of the direct economic impacts which could originate in that sector would then be transmitted through the interindustry linkage structure of the national economy to other sectors in the form of indirect impacts. Industries that could be affected by these impacts include not only those which supply the agricultural sector with goods and services or which purchase agricultural products for further processing and/or resale, but also a host of other industries which are linked either 
vertically or horizontally to the suppliers of agricultural impacts and purchasers of agricultural products.

In that framework, the objectives of this report are:

- To determine what economic methods and models have been developed or need to be developed in order to assess the possible direct and indirect economic impacts of $\mathrm{CO}_{2}$-induced environmental changes in the agricultural sector of the U.S. economy.

- To provide information and recommendations which will enhance DOE's efforts to assess the direct and indirect economic impacts that may be associated with $\mathrm{CO}_{2}$ buildup, not only as they relate to the agricultural sector, but to other sectors of the U.S. economy, as well.

\subsection{ORGANIZATION OF THE REPORT}

This report is divided into seven chapters. Following the Introduction, Chapter 2.0 contains the conclusions and recommendations which are an outgrowth of this research. The conclusions and recommendations contained in this section focus on the following general issues:

- Assessments of the potential of existing agronomic and economic models to provide quantitative answers about the direct and indirect economic impacts associated with $\mathrm{CO}_{2}$ buildup.

- Identification of future research priorities in this area.

Chapter 3.0 has three basic objectives. The first is to provide the unfamiliar reader with a brief introduction about the causes and potential consequences of $\mathrm{CO}_{2}$ buildup on the terrestrial biosphere. The second objective is to identify the various environmental pathways through which $\mathrm{CO}_{2}$ might be expected to impact directly on the production of agricultural commodities by producers in this sector. The third objective is provide the reader with a conceptual framework for understanding the economic relationships through which $\mathrm{CO}_{2}$-induced environmental changes could be transmitted first through the agricultural sector and then into the U.S. economy as a whole. 
There is currently a great deal of uncertainty about the scope and timing of possible $\mathrm{CO}_{2}$-induced changes in the environment and about the economic impacts that could occur as a result of these changes. In that context, Chapter 4.0 is intended to address the question of how research conducted in both of these areas may benefit firms in the private sector by allowing them to adjust to $\mathrm{CO}_{2}$-induced environmental changes before they occur. Based on a discussion of the factors which will influence the behavior of firms under conditions of long-range climatic uncertainty, an effort is made to show how it is possible to value the output of climate research activities in economic terms and to arrange research priorities consistent with the present value of the information that is provided about the economic consequences of $\mathrm{CO}_{2}$ buildup.

Chapters 3.0 and 4.0 are intended to provide a framework for describing the kinds of environmental and economic impacts that might occur as a result of $\mathrm{CO}_{2}$ buildup and the role which economic impact analysis can play in private and public decision-making in the face of uncertainty. Chapter 5.0 initiates the formal analysis within the report of specific models and methods that can be used to assess the direct and indirect economic impacts due to $\mathrm{CO}_{2}$-induced environmental changes within the agricultural sector. The focus of this chapter is on the evaluation of different methods for quantifying the first- and second-order effects of $\mathrm{CO}_{2}$ buildup on the yields of commercial crops. The methods examined in this chapter include:

- panel survey methods;

- statistical crop yield models; and

- crop simulation models.

Chapters 6.0 and 7.0 are devoted to a discussion of the way in which different types of economic models can be used to provide quantitative estimates of the direct economic impacts of $\mathrm{CO}_{2}$-induced environmental changes on the agricultural sector and the indirect impacts of these changes on other sectors of the economy. Chapter 6.0 focuses on models that can be employed in the direct impact analys is phase of this research, namely: econometric and mathematical programing models of the agricultural sector. Chapter 7.0 then examines the way in which the information that is generated 
by these types of models can be used in conjunction with different kinds of macro-models of the U.S. economy to quantify the indirect impacts that will flow from agriculture into other sectors of the economy. Models evaluated in th is section include:

- input-output models;

- input-output based econometric models;

- normative general equilibrium models; and

- multi-regional econometric models.

Finally, Chapter 7.0 examines the way in which these models can be used to provide more detailed insights about the effects of $\mathrm{CO}_{2}$-induced environmental changes in the agricultural sector on the competition for water and land resources and on consumer welfare. 


\subsection{CONCLUSIONS AND RECOMMENDATIONS}

The purpose of this research was to assist DOE in developing methods for assessing the direct and indirect economic impacts due to the effects of increases in the ambient concentration of $\mathrm{CO}_{2}$ on agricultural production. Specifically, the primary objectives were:

- To determine what economic methods and models have been developed or need to be developed to assess the direct and indirect economic impacts of $\mathrm{CO}_{2}$-induced environmental changes in the agricultural sector of the U.S. economy.

- To provide information and recommendations which will enhance DOE's efforts to assess the direct and indirect economic impacts associated with $\mathrm{CO}_{2}$ buildup, not only as they relate to the agricultural sector, but to other sectors of the U.S. economy as well.

\subsection{CONCLUSIONS}

The conclusions contained in this chapter of the report generally fall into three separate categories. The first set of conclusions relates to the identification of the different types of impacts that might occur as a result of $\mathrm{CO}_{2}$-induced environmental changes in the agricultural sector. The second set of conclusions relates to the way in which these impacts can be systematically evaluated using various types of physical and economic models. The final set of conclusions relates to different approaches that can be taken in using these models to provide information about the magnitude of these impacts which will be valuable from a policy perspective.

\subsubsection{Identification of Physical Impacts of $\mathrm{CO}_{2}$ Buildup on Crop Yields}

The agricultural sector is particularly vulnerable to changes in the ambient concentration of $\mathrm{CO}_{2}$ because of the direct ties between many of the constituents of the biosphere and the technology associated with agricultural production. Based on a review of the relevant literature, it is concluded 
that the phenomenon of $\mathrm{CO}_{2}$ buildup has the potential to affect the yields of commercially grown crops in four different ways. These impacts can be classified in terms of first-and second-order effects.

First-Order Effects

First-order effects include:

- The impact of higher ambient $\mathrm{CO}_{2}$ concentrations on crop yields due to increased dry matter production.

- The impact of $\mathrm{CO}_{2}$ buildup on crop yields due to changes in climate. Second-Order Effects

Second-order effects include:

- The impact of $\mathrm{CO}_{2}$ buildup on crop-weed competition due to increased dry matter production by weeds and plants.

- The impact of higher ambient $\mathrm{CO}_{2}$ concentrations on crop yields due to climatically-induced changes in the abundance and geographic distribution of weeds, insects and plant diseases. Joint Effects

In most cases, first- and second-order effects on crop yields can be expected to occur simultaneously. Thus, information about the relationship between individual effects and crop yields will not be sufficient for predicting how all of these effects will interact jointly to change the potential yields of many different crops. At present, the nature of these interactions are not well understood. Until they are, it will not be possible to make accurate statements about the joint impacts of given $\mathrm{CO}_{2}$-induced environmental changes on crop yields.

\subsubsection{Identification of Potential Economic Impacts Due to $\mathrm{CO}_{2}$-Induced Changes in the Environment}

At the current time, there is insufficient information about the first-and second-order effects of $\mathrm{CO}_{2}$-buildup to predict the nature and magnitude of the economic impacts that would occur as a result of an hypothesized change in the ambient concentration of $\mathrm{CO}_{2} \cdot$ Nevertheless, 
analytical models of firm and market behavior can be used to describe, in a general way, the process through which changes in the states of the physical and natural environments work their way from the agricultural sector into the economy as a whole. The information provided by this type of analysis will not reveal answers about the specific types of economic impacts that can be expected as a result of $\mathrm{CO}_{2}$ buildup. However, it can shed light on the potential scope of these impacts, and is helpful in that regard if it can sharpen the focus of future economic research in this area.

Based on this type of analys is it is concluded that the potential economic impacts of $\mathrm{CO}_{2}$ buildup on the agricultural sector can be divided into direct and indirect economic impacts.

\section{Direct Impacts}

The direct economic impacts of $\mathrm{CO}_{2}$-induced environmental change will originate at the level of the individual producer. Potential impacts include:

- Changes in the physical yields of commercially grown crops.

- Changes in the mix of variable inputs (i.e., fertilizer, irrigation water, pesticides) and fixed inputs (i.e., land, machinery, physical structures) used to produce different agricultural commodities.

- Changes in producer profits due to the above two factors.

If the production of a large enough number of producers is affected in some way by $\mathrm{CO}_{2}$ buildup, then market-level impacts will occur. In that case, potential direct economic impacts will include:

- Changes in both the amount of physical output that is produced and the prices which producers receive for their products in those markets where a sizable number of firms are directly affected by $\mathrm{CO}_{2}$-induced environmental changes.

- Changes in the output levels and prices within other agricultural markets in which the production of agricultural comodities is not directly affected by $\mathrm{CO}_{2}$-induced changes in the environment. These spillover effects may occur due to interdependencies in both the supply and demand for different agricultural comodities. 
- Changes in the aggregate profits earned by agricultural producers in those agricultural commodity markets which are directly affected by $\mathrm{CO}_{2}$-induced environmental changes as well as those in which firms are indirectly affected by spillovers from other agricultural markets.

In some cases, analytical models of economic systems can be manipulated to determine the direction of the impact which a change in an exogenous variable, like the ambient concentration of $\mathrm{CO}_{2}$, will have upon the endogenous variables in the model, such as physical outputs, prices and profits. This was attempted. Based on the results of this analysis, it can be concluded that neither the direction nor the magnitude of the impacts of $\mathrm{CO}_{2}$-induced environmental changes on agricultural output, prices and profit levels can be determined a priori without specific information regarding: 1) the technical relationships governing the impact of changes in the state of the environment on commodity production, and 2) the effect which a change in the price of one agricultural commodity will have upon the individual prices of other agricultural commodities. Thus, even if the impacts of $\mathrm{CO}_{2}$ buildup on the environment were known with certainty--which they are not--it would still not be possible to predict the economic impacts associated with hypothesized changes in the state of the environment. To do so requires models which can first translate the impacts of $\mathrm{CO}_{2}$ on the environment into changes in the yields of specific crops and then translate the effect of changes in output in individual agricultural markets into changes in output, price and profit levels within the web of markets that define the agricultural sector as a whole.

\section{Indirect Impacts}

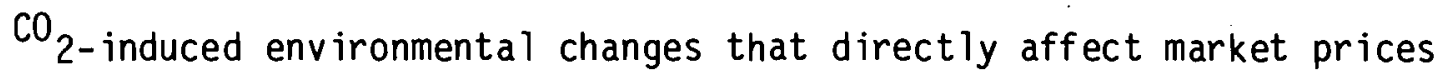
and output levels in the agricultural sector may be transmitted through the interindustry linkage structure of the national economy to other sectors in the form of:

- Changes in the prices, output levels and profits in markets which supply the agricultural sector with land, labor, capital and other inputs required to produce agricultural comodities. 
- Changes in the prices, output levels and profits in markets which directly purchase agricultural commodities for further processing and/or resale.

- Changes in the expenditure patterns of consumers for a great many goods due, directly, to changes in the price of food and, indirectly, to changes in the price of other goods and services which are produced by industries that are connected to the agricultural sector through forward and backward linkages.

- Changes in the prices, output levels and profits in markets that are connected either horizontally or vertically to industries that supply inputs to, or purchase the outputs of, the agricultural sector.

Impacts from Foreign Trade

$\mathrm{CO}_{2}$ buildup is a global phenomenon. Therefore, the same general kinds of direct and indirect economic impacts that could occur in the U.S. could also occur simultaneously in many other nations--although the magnitude and direction of these changes will almost certainly be different from country to country. Potential impacts on U.S. agriculture due to $\mathrm{CO}_{2}$-induced environmental changes that will affect agricultural production in other nations include:

- Changes in the international trade prices of world food commodities.

- Changes in the competitive position of U.S. agriculture in world food markets.

It is concluded, therefore, that the indirect impacts due to $\mathrm{CO}_{2}$-induced environmental changes in agricultural production have.the potential to be extremely pervasive. However, because $\mathrm{CO}_{2}$-induced price changes in one market or sector will simultaneously affect the equilibrium prices and output levels in many other sectors, it is very difficult to make a priori statements about either the magnitude or direction of these impacts on the economic well being of producers and consumers in the economy as a whole. The same general conclusions also apply in the context of international trade. 


\subsubsection{Methods for Assessing the Impacts of $\mathrm{CO}_{2}$ on Climate}

This report did not examine in detail, the different types of $c l$ imate models that can be used to provide information about the impacts of increases in the ambient concentration of $\mathrm{CO}_{2}$ on climate. However, an effort was made to evaluate the consistency between the meteorological information provided by general circulation models (GCMS) and the information requirements of models used to evaluate the impact of $\mathrm{CO}_{2}$-induced climate change on crop yields. In general, it was concluded that, while GCMs can provide the necessary type of meteorological information required to drive different types of crop yield models, improvements need to be made in the following areas before GCMs will be fully compatible with crop yield models:

- Geographic Features--GCMs predict climate change over an artificial geographic domain, whereas the effects of climatic change on crop yields must be consistent with specific regions that are used in forecasting the effects of $\mathrm{CO}_{2}$-induced climate change in the agricultura? secto".

- Model Vali-ation--Because GCMs simulate climate change over an idealized geographic domain, they cannot be validated statistically against existing meteorological data bases; consequently, neither the accuracy of these models nor the yield forecats produced in conijunction with crop yield models can be deternined statistically.

- Regional Delineation--The geographic resolution of crop yield models can be made compatible with the geographic resolution of GCMs; however, the accuracy of the meteorological information supplied by GCMs at the current level of resolution (a $5^{\circ}$ square grid) is considered to be poor.

- Frequency--The frequency of the meteorological information required by crop yield models ranges from annual averages to daily values, whereas state-of-the-art GCMs can, at best, provide meteorological incorntion on approximately a monthly basis with questionable accuracy. 


\subsubsection{Methods for Assessing the Impacts of $\mathrm{CO}_{2}$-Induced Environmental Changes on Crop Yields}

Three different methods for quantifying the impacts of $\mathrm{CO}_{2}$-induced environmental changes on crop yields were examined. The methods chosen were selected based on their ability to provide crop yield forecasts for a number of different crops in a multi-regional setting. These methods included:

- Panel Survey Approach--which is a method that involves the statistical manipulation of expert opinions about future climatological changes and crop response to meteorological conditions in order to arrive at projected estimates of crop yields.

- Statistical Yield Models--which employ parametric statistical methods, such as multivariate regression analysis, to estimate the parameters of yield response functions using historical and/or experimental data on crop yields and meteorological variables.

- Simulation Models--which are mathematical representations of the physical, chemical and physiological mechanisms underlying plant growth processes specific to individual crops, and are therefore generalizable to any set of environmental conditions that might be encountered.

Major conclusions regarding the value of these methods for predicting how $\mathrm{CO}_{2}$-induced changes in the environment will affect crop yields are as follows:

\section{Panel Survey Method}

The primary advantages of this approach are its efficiency and flexibility. The efficiency of this method refers to the fact that it can be utilized in a fairly rigorous statistical framework to simulate the potential impacts of many different types of $c l$ imatic changes on region-specific crop yields, given a relatively small amount of hard information about future cl imate changes. The approach is flexible in that it can be adapted for use with existing climate and agricultural sector models without having to modify 
the form in which climate information is provided by GCMs or the region-crop coverage contained within agricultural sector models. The disadvantages associated with this approach are, first, that it relies on subjective estimates of the relationship between changes in meteorological variables and crop yields; second, that the number of meteorological variables used in the analys is must be kept fairly small (i.e., annual growing season temperature and precipitation, by region); and third, that it would be difficult to extend this type of analys is to an assessment of joint first-and second-order environmental effects on crop yields.

\section{Statistical Crop Yield Models}

While this report did not identify all of the statistical crop yield models that have been constructed in the last 20 or so years, the results of a limited search of the literature suggest that the region-crop coverage of existing models is not compatible with that contained in multi-regional agricultural sector models. Therefore, if this approach is used to assess the impact of $\mathrm{CO}_{2}$-induced environmental changes on crop yields, these discrepancies will have to be identified and eliminated by estimating new models. The primary advantage of pursuing this approach is that individual models can be estimated relatively quickly from existing data bases, with a demonstrated degree of accuracy which can be measured in statistical terms. In addition, the information requirements of these models can be met satisfactorily by existing GCMs, provided that simple procedures are developed to eliminate disparities between the regional delineations used in the two types of models.

There are, however, three primary drawbacks associated with using these types of models to simulate the effects of $\mathrm{CO}_{2}$ buildup on crop yieilds. The first two of these are related to the fact that, because the parameters of these models are derived empirically, the results obtained are usually specific to the data base used in estimating the model. Thus, it will probably be necessary to use different regional models for each crop, although newer developments in the field suggest that these models can be made more genera 1. In addition, the accuracy of the yield forecasts produced by these models under simulated conditions tends to decrease as the values assigned to 
the meteorological variables depart from the observed values used to estimate model parameters. The seriousness of this problem will depend on the relative severity of expected $\mathrm{CO}_{2}$-induced climatic changes on a region-by-region basis. A final drawback associated with statistical crop yield models is that they can only be used to assess the impact of $\mathrm{CO}_{2}$-induced climate change on crop yields and not the full range of first- and second-order environmental effects.

\section{Crop Simulation Models}

The theory that underlies the structural features of these models suggest that they are the best-suited of all the methods for simulating the whole plant response to a wide variety of first-and second-order $\mathrm{CO}_{2}$-induced environmental changes, both singly and jointly. In contrast to statistical crop yield models, crop simulation models are designed specifically to forecast yield changes over a wide range of environmental conditions. This not only eliminates the need for individual regional models, but should also lead to greater accuracy in simulating the impacts of $\mathrm{CO}_{2}$-induced climate change on crop yields than is possible with statistical crop yield models. A final comparative advantage of this method over others surveyed is that it can be adapted to simulating the effects of non-climatic environmental changes on crop yields.

Unfortunately, the realities of crop simulation models fall somewhat short of their theoretical advantages. While the crop coverage of these models is fairly extensive, the accuracy of individual models varies tremendously. In some cases, statistical crop yield models are substantially more accurate than crop simulation models. A second problem is the amount of research effort required to improve the accuracy of existing models or to construct new models. Whereas it might take a single researcher a month or two to collect the necessary data and estimate the parameters of a statistical crop yield model, it could easily take a team of scientists and support staff two or three years to develop a crop simulation model from the ground up. $A$ final set of problems relates to data mismatches between crop simulation models and both climate and agricultural sector models. Crop simulation models are designed to provide field-specific yield forecasts usirig daily 
values for solar radiation precipitation and temperature. GCMs cannot provide meteorological information at this level of spatial and temporal resolution nor can agricultural sector models accept yield information at the field level. Therefore, before crop simulation models can be used effectively in conjunction with climate and agricultural sector models systematic methods will have to be derived for resolving these inconsistencies through appropriate data aggregation/disaggregation techniques.

\subsubsection{Methods for Assessing the Direct Economic Impacts of $\mathrm{CO}_{2}$-Induced Changes in the Environment}

This report examined three different types of agricultural sector models that can be used to assess the direct economic impacts of $\mathrm{CO}_{2}$-induced environmental changes within the agricultural sector. These types of models inc luded:

- Positive (Econometric) Models--which employ advanced multivariate regression methods (e.g., econometrics) to estimate the parameters of commodity-specific supply and demand equations using historical data.

- Normative (Mathematical Programing) Models--which employ numerical optimization methods to allocate available resources such as 1 and, fertilizer and irrigation water among competing agricultural production activities within the agricultural sector in a manner that 1) satisfies the objective function of the model (e.g., maximize net returns or minimize production loads) and 2) is consistent with a set of technological relationships relating resource use to output. Normative agricultural sector models differ from the ir positive counterparts conceptually in that they attempt to describe how economic agents should behave in the aggregate if they wish to achieve certain objectives, whereas positive models attempt to predict the actual behavior of economic agents.

- Agricultural Trade Models--which usually consist of a series of riational or multi-national agricultural sector models linked together through the exchange of agricultural commodities in order 
to satisfy commodity and country specific demands for these goods. Agricultural trade models can be designed around either an econometric or mathematical programing framework.

Major conclusions regarding the value of these models to assess the direct economic impacts of $\mathrm{CO}_{2}$-induced environmental changes in the agricultural sector are as follows:

\section{Econometric Models}

Existing multi-regional models of the agricultural sector can be used to assess the impacts of $\mathrm{CO}_{2}$-induced yield changes on commodity prices, production, agricultural land use and producer profits on a regional basis, over time. These models can be modified to incorporate the effect of changes in climate on crop yields directly within the yield equations contained in the model. This would partially alleviate the need to generate yield estimates from separate crop yield models. The primary weakness of econometric models in the current context is that their ability to accurately forecast the impacts of $\mathrm{CO}_{2}$ buildup on agricultural production over very long periods of time is highly questionable. There are two reasons for this. First, these models cannot predict future changes in technology and consumer preferences, nor the impact of these changes on the way in which producers and consumers will adjust to $\mathrm{CO}_{2}$ buildup. Secondly, because these models are empirical in nature, they tend to emphasize short-run adjustments on the part of producers to changes in the environment, as opposed to the kind of long-run adjustments that can be expected to occur in response to $\mathrm{CO}_{2}$ buildup. This is a serious prohlem because long-run adjustments are less costly to society than short-run adjustments. This is due to the fact that land, labor and capital are freer to move to alternative economic opportunities in the long-run than in the short-run. For both of these reasons, econometric models will probably overstate potential social costs and understate potential social benefits connected with $\mathrm{CO}_{2}$ buildup. It is also likely that econometric models will tend to understate regional shifts in the distribution of crop production and farm income which might also occur in response to changes in the ambient concentration of $\mathrm{CO}_{2}$. 


\section{Normat ive Mode ls}

Normative models of the agricultural sector can also be used to assess the impacts of $\mathrm{CO}_{2}$ buildup on commodity prices, production, agricultural 1 and use and profits on a regional basis. While normative models are generally static in character, adaptive recursive versions discussed in Chapter 7 can be used to simulate the impacts of $\mathrm{CO}_{2}$-induced environmental changes over time, as well. Specific advantages that normative agricultural sector models have over econometric models are that they contain a larger number of regions, they provide more information about input usage, specifically, fertilizer, water and soil loss, and that the normative character of the information obtained from these models more nearly represents the outcome of long-run adjustment processes than do the results provided by econometric models. However, normative models are no more capable of predicting changes in technology and consumer preferences than are econometric models, so that the accuracy of long-range economic forecasts produced by these models will also be questionable. In addition, normative models do not usually contain yield equations; therefore, the effects of $\mathrm{CO}_{2}$-induced climate changes on crop yields cannot be simulated directly within the structure of the model as would be the case with an econometric model. A final drawback of normative models is the relatively high cost of conducting simulation runs compared to econometric models.

\section{Agricultural Trade Models}

Neither econometric nor normative models of the agricultural sector are structured to examine the effects of $\mathrm{CO}_{2}$-induced environmental changes in other nations upon world agricultural trade. These impacts can be assessed by using existing multi-country agricultural trade models or by linking together agricultural sector models for many different nations through a general equilibrium framework. To utilize the former type of model in an economic assessment of the impacts of $\mathrm{CO}_{2}$ buildup, crop-country specific yield equations will have to be estimated and integrated within the structural equations. Methods for linking a large number of individual country models in a world trade framework are currently under development by the Institute for 
International Applied Systems Analysis. However, it is not known what modifications would be required, if any, to this system in order to make it compatible with the demands of a $\mathrm{CO}_{2}$ economic impact assessment since the project is not well advanced and the date for its completion is unclear.

\subsubsection{Methods for Assessing the Ind irect Economic Impacts of $\mathrm{CO}_{2}$-Induced Changes in the Environment}

Two different approaches can be used to assess the indirect economic impacts of $\mathrm{CO}_{2}$ buildup. The first approach would involve embedding a very detailed agricultural sector model directly within the structure of a highly disaggregated general equilibrium model of the U.S. economy. The second approach would involve first simulating the effect of the $\mathrm{CO}_{2}$-induced environmental changes on the agricultural sector and then inserting the relevant commodity output and price information that is obtained from the agricultural sector model into a linked macro-model of the U.S. economy in the form of output and price constraints. It is concluded that the second of these two methods--the so-called linked model approach-- is the best method for assessing the indirect economic impacts of $\mathrm{CO}_{2}$ buildup. This approach is favored for two reasons:

- The region-crop detail contained in existing multi-regional agricultural sector models is far greater than that which could reasonably be built into an embedded model of the agricultural sector.

- The benefits of embedding a very detailed model of the agricultural sector into a general equilibrium model are not yet clear, while the cost of doing so is, relatively speaking, quite large. Under these conditions, it is preferable to wait until the benefits of building a more sophisticated model are readily apparent.

The different types of macroeconomic models examined in this report included:

- Input-0utput Models--which divide the economy into a large number of disaggregated industries and then establish the magnitude of the flows of goods and services between industries based upon a matrix 
of technical coefficients describing the raw materials which each industry in the economy requires from every other industry in order to produce a unit of output. By manipulating the matrix of technical coefficients through elementary methods of matrix algebra, it is possible to use an input-output model to determine how a change in the final demand for the output of one or more industries affects the gross output of other industries.

Input-Output Based Econometric Models--which combine the industry accounting system used in conventional input-output models with statistically estimated demand functions for final goods and services and primary inputs to production in a manner that enables these models to better simulate the substitutions that consumers and producers are likely to make in response to changes in economic conditions for final goods and services and primary inputs to production, such as energy, labor and capital.

- Normative General Equilibrium Models--which are basically non-linear programing models of the economy containing essentially the same structural components as input-output based econometric models, but which provide normative solutions for the endogenous variables within the model as opposed to positive solutions.

- Multi-Regional Econometric Models--which are designed to show how changes in the non-local demand for regional goods and services affect industry output and employment, along with income and population growth, in many different regions of the U.S., usually of the country or standard metropolitan statistical region (SMSA) level. Input-output methods were rejected as inappropriate because prices play no role in determining the allocation of resources to different industries within these models, making it impossible to determine how changes in the prices of agricultural commodities that might be caused indirectly by $\mathrm{CO}_{2}$ buildup will affect gross income in other sectors of the economy. Conclusions regarding the suitability of the remaining three types of models for assessing the indirect economic impacts associated with $\mathrm{CO}_{2}$-induced environmental changes in the agricultural sector are as follows: 


\section{Input-Output Based Econometric Models}

These types of models represent the best available alternative for obtaining quantitative estimates of the indirect impacts associated with $\mathrm{CO}_{2}$-induced environmental changes in the agricultural sector, over time. Methods for using these models in conjunction with agricultural sector models have already been developed in a different research context. The incorporation of a system of econometrically estimated consumer demand functions also makes it possible to use these models to provide theoretically "exact" measures of changes in consumer welfare due to $\mathrm{CO}_{2}$-induced changes in the prices of many different goods and services. However, existing models cannot be used effectively to investigate impacts on the interindustry distribution of water and 1 and resources, although several potential alternatives exist for partially remedying this deficiency. As a forecasting instrument, input-output models are designed to adjust product prices in response to cost-based changes in industry productivity that are projected to occur over time. Thus, these models are capable of at least partially duplicating long-run adjustment processes that may occur in response to $\mathrm{CO}_{2}$ buildup. However, future productivity changes are modeled on the basis of past trends over the last thirty or so years. In that regard, these models are no more capable of predicting future changes in technology and consumer tastes than any of the other models examined in this report.

\section{Normative General Equilibrium Models}

These models are potentially of great benefit because they provide competitive, general equilibrium solutions that would be consistent with an optimal allocation of resources with in the macroeconomy in response to $\mathrm{CO}_{2}$-induced environmental changes in the agricultural sector. As such, a model of this type is better suited than an input-output based econometric model for showing the full capacity of the economy to adjust to these changes in the environment, given current technology and preferences. In addition, it is possible to incorporate into these models the same, or even a greater level of detail, regarding interindustry transactions. Finally, these models are explicitly resource based and consequently can be used to examine the impact 
of $\mathrm{CO}_{2}$-induced environmental changes within the agricultural sector on the competition for both 1 and and water resources. The major drawback to the use of such a model in future assessments is that it would be necessary to build a prototype from the ground up, since no actual model of the U.S. macroeconomy currently exists. However, the structural features of normative general equilibrium models have been developed and the results obtained from small scale models are encouraging. It is concluded that a larger model of the U.S. economy would represent a highly complementary approach to the use of input-output based econometric models.

\section{Multi-Regional Econometric Models}

These models represent the only available means for providing quantitative answers about the magnitude and scope of the possible impacts of $\mathrm{CO}_{2}$ buildup on regional econometric activity. While these models are not a substitute for either input-output based econometric models or normative general equilibrium models, they can be used in conjunction with either of the two, along with the information supplied by a multi-regional econometric model, to assess the possible impacts of $\mathrm{CO}_{2}$ indirect environmental changes on industry output and employment income and population at the county or SMSA level. This type of model would be particularly valuable in showing how possible long-run changes in the spatial distribution of agricultural production might affect rural economics which could be especially vulnerable to these types of distributional impacts. The major weakness of these models lies in their empirical nature: because model parameters are estimated on the basis of recent historical data these models may provide misleading answers about the ability of individual regions to adapt, structurally, to the impacts of $\mathrm{CO}_{2}$ buildup.

\subsubsection{Strategies for Assessing the Economic Impacts of $\mathrm{CO}_{2}$-Induced Changes in the Environment}

There are essentially two reasons for developing a capability to predict the long-range effects of $\mathrm{CO}_{2}$ buildup on the environment and the impacts of these changes on the economy. The most obvious reason is to be able to determine the net benefits that will accrue to society as a result of instituting alternative policies to check the current buildup of $\mathrm{CO}_{2}$ in the 
atmosphere. This is consistent with the regulatory power of the public sector that is traditionally invoked whenever private markets do not provide appropriate incentives for firms to consider the impacts of their production decisions on society. The second reason is to provide economic agents in the private sector with information that will enable them to mitigate the adverse impacts and take advantage of the favorable impacts of future climate changes, before they occur. Making this information available to the private sector is a legitimate public purpose, provided that firms lack the appropriate economic incentives to produce this information efficiently.

There are two basic obstacles that stand in the way of providing accurate forecasts about the potential economic impacts of $\mathrm{CO}_{2}$ buildup. The first of these is that state-of-the-art climate models cannot be empirically validated. Thus, the accuracy of the forecasts provided by these models cannot be determined statistically in the same manner that this can be done with the forecasts generated by statistical yield models and crop simulation model. The second obstacle is that economic models cannot, in general, predict future changes in technology and consumer tastes, nor the impact of these changes on the way in which producers and consumers adjust to $\mathrm{CO}_{2}$ buildup. Since some of these changes in technology and consumer preferences will foreseeably represent adaptive responses to $\mathrm{CO}_{2}$ buildup, the accuracy of the long-range forecasts produced by both agricultural sector models and macroeconomic models will be extremely difficult to defend. This will be true regardless of the progress that is made in climate modeling, since the problem lies not in the accuracy of the input data that is used to simulate the inpacts of $\mathrm{CO}_{2}$ buildup on crop yieids, but rather in the inability of science to predict either the practical results of human ingenuity (technological changes) or changes in human values (tastes).

Consequently, if economic assessments are to be performed, a way of using economic models to shed light on the potential impacts of $\mathrm{CO}_{2}$ buildup must be found as a substitute for trying to make precise predictions of future economic conditions 50 to 100 years or more from the present. It is concluded that there are at least two possible alternatives available for doing this, each of which can be viewed as a different research, or forecasting, strategy. 


\section{Instantaneous Adjustment Strategy}

This strategy attempts to answer the question: what would happen if $\mathrm{CO}_{2}$-induced environmental changes occurred tomorrow, rather than in 50 to 100 years? The rationale underlying this strategy is that, by imposing this kind of a sudden, exogenous shock on the agricultural sector, one can examine how that sector would respond given existing technology and consumer preferences and under existing short-run technological and institutional constraints. The results obtained from this procedure would represent a "worst case" impact scenario. In the long-run, much less costly adjustments to $\mathrm{CO}_{2}$ buildup can be expected, hoth because of technological changes within the agricultural sector and due to the greater mobility of land, labor and capital given a long enough period of time to optimally adjust to these changes. Such an approach can be beneficial because:

- It will provide a lower bound on net societal benefits and/or an upper bound on the costs of $\mathrm{CO}_{2}$ buildup.

- It can point to potential trouble spots in the agricultural sector which can then be assessed more closely in terms of subjective judgments about potential future problems in these areas.

- It can provide information about the types of environmental changes which are likely to cause the most or least severe problems, and additional research can be focused on assessing the probabilities of these events actually occurring.

\section{Dynamic Adjustment Strategy}

The basic problem with the static approach to simulating the economic impact of $\mathrm{CO}_{2}$ buildup is that it is not very realistic. The effects of $\mathrm{CO}_{2}$ buildup on crop yields will occur gradually over time, not suddenly. While the static strategy may be useful as a possible indicator of the severity of the impacts associated with $\mathrm{CO}_{2}$ buildup, it does not provide any insight about the way in which the economy might adjust, differentially, to $\mathrm{CO}_{2}$ buildup over time. In order to do this, dynamic models can be used to examine how today's economy will respond to gradual changes in the state of the environment. In that way, a better understanding can be gained about the 
timing and sequence of economic impacts. Again, this approach does not imply that these impacts will actually occur, since the adjustments that the economy will be able to make to changes in the environment will invariably be consistent with the much greater mobility of resources in the long-run. Nevertheless, this approach will make it possible to examine the temporal aspect of resource mobility in an economic setting of partial, but not full adjustment.

An important feature of both these strategies is that they can be used immediately to analyze the sensitivity of the agricultural and nonagricultural sectors of the economy to a range of hypothetical $\mathrm{CO}_{2}$-induced changes in the environment, rather than waiting for more accurate climate scenarios. This is a valuable type of exercise because it can be used to provide information on a current basis about the types of $c l$ imatic and other environmental changes which appear most (or least) likely to create potentially severe economic impacts. This information can, in turn, be used to refocus research upon specific problems in specific regions in order to provide more accurate information about the possible economic consequences of $\mathrm{CO}_{2}$ buildup.

\subsection{RECOMMENDATIONS}

Based on the conclusions of this report, the following recommendations are provided as a basis for developing a methodology to assess the direct and indirect economic impacts of $\mathrm{CO}_{2}$-induced environmental changes originating in the agricultural sector of the U.S. economy.

\subsubsection{Research Scope}

It is recomended that current research efforts in developing a methodology for assessing the direct and indirect impacts due to $\mathrm{CO}_{2}$-induced environmental changes in the agricultural sector be focused in the following directions:

- Use currently existing models to assess the sensitivity of both the agricultural and nonagricultural sectors of the economy to a wide range of simulated changes in crop yields. 
- Assess the ability of the economy to adjust to both sudden and gradual changes in regional crop yields using positive as well as normative models of the agricultural sector and the U.S. macroeconomy.

- Incorporate more specific information about the expected first-and second-order effects of $\mathrm{CO}_{2}$ buildup on the environment into the above analysis as this information becomes available through on-going research on climate, and plant responses to changed environmental conditions.

\subsubsection{Development of Climate Models}

Much can be learned fairly quickly about the sensitivity of the U.S. economy to both sudden and gradual $\mathrm{CO}_{2}$-induced climate changes using existing information. However, long-range research goals that should be pursued in the field of climate modeling in order to achieve more accurate forecasts include:

- The development of GCMs that can simulate climatic changes over the actual physical geography of existing regions and which can be validated using empirical data on current climate from these regions.

- Improving the accuracy of GCM-based climate forecasts at existing levels of spatial ( $5^{\circ}$ square grid) and seasonal (23-30 days) resolution.

- The development of a capability to provide climate forecasts in a stochastic framework.

\subsubsection{Selection of Methods for Assessing the Impacts of $\mathrm{CO}_{2}$-Induced} Environmental Changes on Crop Yields

There is a considerable amount of disparity between the ability of existing methods to simulate the impacts of $\mathrm{CO}_{2}$-induced climate change on crop yields and the remaining types of first- and second-order environmental affects. Therefore, it is recommended that the development of a capability to simulate these impacts consist of the following stages: 
- For the immediate future, it is recommended that the influence of variations in meteorological variables on crop yields should be incorporated directly within the framework of a multi-regional econometric agricultural sector model by re-estimating the yield equations of the model using readily available historical data bases created by the U.S. Department of Agriculture.

- In the meantime, efforts should be undertaken to fund research related to improving the generality of statistical crop yield models and to substitute these new yield equations for those previously estimated in the initial research/simulation stage.

- At the same time, long-term research should be focused on developing a capability to ultimately replace these submodels with a system of crop simulation models to predict the full range of first-and second-order environmental effects on crop yields for all crops contained within the agricultural sector model used in the analysis. Elements of this research would include improving the coverage and statistical accuracy of these models, with particular emphasis upon modeling the effect of $\mathrm{CO}_{2}$ on the production of dry matter by commercially grown plants and weeds and on the interactions between crop development and pest abundance using experimental data obtained from growth chamber experiments.

\subsubsection{Selection of Economic Models for Conducting Impact Assessments}

In order to assess the economic impacts associated with $\mathrm{CO}_{2}$-induced environmental changes in the agricultural sector in a cost-effective manner, it is recommended that an existing multi-regional agricultural sector model be linked together in a quasi-general equilibrium framework until the benefits of constructing a single large general equilibrium model of the economy have been established. Consistent with this recommendation, the following models have been selected as the best available candidates for assessing the economic impacts of $\mathrm{CO}_{2}$ buildup: 
Agricultural Sector Models

- AGSIM--a multi-regional econometric model containing 51 region-crop combinations (plus livestock), versions of which are now held by the Environmental Protection Agency (EPA) and Texas A\&M University.

- ISU/NRE National Regional Linear Programming Model (CARD CT) -. a large multi-regional, price endogenous mathematical programing model containing more than 300 region-crop combinations (plus livestock), developed jointly by the U.S Department of Agriculture and the Center for Agricultural and Rural Development at Iowa State University.

Agricultural Trade Models

- Grain, 0ilseed and Livestock Model (GOL)--a multi-commodity, multi-country world agricultural model featuring 28 different country groups, 12 major commodities linked together in a price-endogenous spatial equilibrium framework, developed by the U.S. Department of Agriculture.

Macro- and Multi-Regional Econometrics Models

- Battelle Forecasting System (FORSYS)--an input-output based econometric model of the U.S. economy containing 107 production sectors and 54 disaggregated demand sectors, developed by Battelle Memorial Institute.

- Metropolitan and State Regions Model (MASTER)--an SMSA-based multi-regional econometric model developed by the Pacific Northwest Laboratory for the Department of Energy which is capable of forecasting activity levels, employment and income for 13 industries, plus regional employment and population change.

- Normative General Equilibrium Models (NGE)-- while no operational NGE model of the U.S. economy is currently available, the feasibility of building such a model should be explored further.

\subsubsection{Modifications to Existing Models}

In order to adapt existing models to the unique demands of assessing the direct and indirect economic impacts due to $\mathrm{CO}_{2}$-induced environmental 
changes originating in the agricultural sector, the following technical modifications are recommended:

Mod if ications to AGSIM

- The yield equations within the AGSIM model should be re-estimated using meteorological data in order to make the effect of weather on yields endogenous to the model.

- The acreage response equations within AGSIM should be re-estimated to include the effect of climate-induced risk on planting decisions. Modifications to GOL

- Yield equations for each country-crop combination included in the model should be estimated and integrated within the structure of the mode 1 .

Mod if ications to FORSYS

- Primary resources in this model should be expanded to include both water and 1 and resources; however, alternative methods for doing this must be first examined more carefully in light of possible data limitations. 


\section{$3.0{ }_{2}$ AND THE ECONOMICS OF ENVIRONMENTAL CHANGE IN AGRICULTURE}

The primary objectives of this chapter are two-fold. The first is to identify, in general terms, the various ways in which $\mathrm{CO}_{2}$-induced changes in both the physical and natural environment may affect the yields of commercial crops. The second objective is to systematically outline the cause and effect relationships by means of which these environmental changes might affect the various allocation decisions made by producers and consumers both in the agricultural sector and the U.S. economy as a whole. In pursuing these objectives no attempt will be made to speculate about the way in which $\mathrm{CO}_{2}$-induced environmental changes will actually affect either crop yields or the behavior of economic agents. Rather, the central purpose that underlines both of these objectives is to provide a framework for identifying the mechanisms through which changes in the atmospheric concentration of $\mathrm{CO}_{2}$ will be transmitted from the environment to the agricultural sector and from there into other sectors of the U.S. economy.

This chapter is divided into four main sections. The first of these is a fairly general introduction to the nature of the $\mathrm{CO}_{2}$ problem which outlines the main points of consensus within the scientific community regarding the magnitude and climatic impacts of further increases in the concentration of $\mathrm{CO}_{2}$ in the atmosphere. Readers who are already familiar with this background information may wish to skip to the next section. Section 3.2 identifies the pathways through which $\mathrm{CO}_{2}$-induced changes in the environment can be expected to influence agricultural production. The next section makes use of a series of analytical models to show how these changes in the environment will in theory affect not on $1 y$ the production decisions of individual producers, but also the determination of equilibrium prices and output levels in many different agricultural commodity markets. Section 3.4 examines the mechanisms by which the indirect impacts of $\mathrm{CO}_{2}$-induced environmental changes will be transmitted to other sectors of the economy through forward and backward linkages. Finally, Section 3.5 contains a nontechnical sumary of the theoretical development outlined in the two previous sections. 


\subsection{THE $\mathrm{CO}_{2}$ PROBLEM}

Carbon dioxide is a natural constituent of the earth's atmosphere. Concentrations of $\mathrm{CO}_{2}$ in the atmosphere are regulated by the carbon cycle, a process which describes the exchange of carbon between the atmosphere, the terrestrial biosphere and the oceans. Prior to the industrial revolution (c. 1800), it appears that the natural sources and sinks of $\mathrm{CO}_{2}$ were in a near equilibrium, or steady-state condition. It has been estimated that, during the early part of the nineteenth century, the concentration of $\mathrm{CO}_{2}$ in the earth's atmosphere was about 290 parts per million by volume (ppmv) (Siegenthaler and Oeschger 1978), although some reports have suggested concentrations as $10 \mathrm{w}$ as 268 to $270 \mathrm{ppmv}$ (Stuiver 1978; Wilson 1978). Until 1958, ambient $\mathrm{CO}_{2}$ was not measured on a regular basis. However in that year, Keeling and his associates began monitoring $\mathrm{CO}_{2}$ at two locations, Mauna Loa, Hawaii and the South Pole (Keeling et al. 1976). These measurements show that the concentration of $\mathrm{CO}_{2}$ in the atmosphere has increased continually from 315 ppmv in 1958 to about 340 ppmv in 1980, and is currently rising at a constant rate of about 1.5 ppmv per year.

There is almost universal agreement within the scientific community that the sharp rise in the concentration of $\mathrm{CO}_{2}$ in the atmosphere that has taken place in the last 100 to 150 years has been largely due to the accelerated burning of fossil fuels, although other anthropogenic sources of carbon cannot be overlooked (Sagan et a1. 1979). It has been estimated that, during this period, about 150 to 170 billion metric tons (BMT) of fossil fuel carbon were released into the atmosphere, while the amount of carbon present in the atmosphere rose from about 620 BMT (292 ppmv) to 709 BMT (334 ppmv) (National Research Council 1979). In other words, the increase in the amount of carbon in the atmosphere that has taken place since the early part of the nineteenth century represents about one-half of the carbon released by fossil fuel burning. This trend appears to be continuing. Currently, fossil fuel carbon discharges are running about 5 BMT per year, while the corresponding increase in the amount of atmospheric carbon is about 2 to 3 BMT per year (U.S. Department of Energy 1980). 
Projections of future $\mathrm{CO}_{2}$ concentrations in the atmosphere vary widely, depending upon the assumptions that are made about (1) the magnitude of carbon fluxes and the disposition of sources and sinks in the global carbon cycle, (2) the rate at which fossil fuels will continue to be burned, and (3) the distribution of this consumption across different types of fossil fuel (Chen et al. 1980). At present, there is a considerable gap in the sophistication of the models used to make projections of atmospheric $\mathrm{CO}_{2}$ (Broecker et al. 1980). However, the state-of-the-art appears to be such that the current range of disagreement between these models is not very great under the most plausible fossil fuel use scenarios (Council on Environmental Quality 1981). Recent projections published by the Council on Environmental Quality (CEQ) using a fairly simple model suggest that it will take between 44 and 194 years for the atmospheric concentration of $\mathrm{CO}_{2}$ to reach a magnitude double that of pre-industrial estimates, depending on the assumed rate of growth of fossil fue 1 use. At current rates, this doubling would occur in slightly less than 70 years, according to CEQ est imates.

With a few exceptions (Idso 1980; Newe 11 and Dopplick 1979) there seems to be little disagreement within the scientific community that substantial increases in the concentration of $\mathrm{CO}_{2}$ in the atmosphere will result in a rise in global mean temperatures. This warming process, sometimes referred to as the greenhouse effect, is fairly simple to explain. The earth is generally thought to remain in a state of thermal equilibrium by radiating back into space an amount of energy equal to the energy absorbed from the sun plus an additional, but very smal1, amount of energy which is produced through fossil fuel combustion. The short wave solar radiation that passes through the atmosphere to the earth is relatively unaffected by the presence of $\mathrm{CO}_{2}$. However, the radiation released from the colder earth is in the longer, infrared wave lengths, and it is this energy which is partially absorbed by $\mathrm{CO}_{2}$. Other things being equal, an increase in the infrared capacity of the atmosphere will cause the temperature of the earth's surface and the adjacent atmosphere to increase until the emission of radiation from the earth again equals the amount of solar energy that has been absorbed. Hansen et al. (1981) have compared the change in temperature due to the greenhouse effect to 
depth of water in a leaky bucket with a constant inflow rate. If the holes in the bucket are made smaller, then both the depth and the pressure of the water in it will increase until the rate of outflow equals the rate of inflow.

While the radiative effects of increasing the concentration of $\mathrm{CO}_{2}$ in the earth's atmosphere are well understood, determining the specific impacts of the greenhouse effect on the global climate system turns to to be a very complicated task. Efforts to explain what a rise in global mean temperatures will mean in terms of the magnitude and geographic distribution of temperature, precipitation and evaporation, and cloud cover have proceeded along two different lines. The first type of approach involves reconstructing past climates during periods when the earth was generally warmer than it is today. Periods that have been singled out for analysis include those in the recent past, between 1925 and 1974 (Wigley et al. 1980) and 1935 to 1950 (Ratcliffe et al. 1978; Flohn 1980), the early Middle Ages from about 900 to 1200 (Lamb 1977 , 1979; Barry et al. 1977), and the Holocene warm period which peaked around 6000 years ago (Flohn 1980; Kellog 1978, 1979).

The second approach involves the simulation of climatic changes due to $\mathrm{CO}_{2}$ increases in the atmosphere through the use of mathematical models of varying degrees of sophistication. The three general types of climate models that have been used to do this are radiative convective models (Ramanathan and Coakley 1978), latitudinally varying energy balance models (Ramanathan et al. 1979) and global circulation models (GCMs) such as those developed by Manabe and Wetherald $(1975,1980,1981)$ at the National Oceanographic and Atmospheric Administration's (NOAA) geophysical Fluid Dynamics Laboratory. The comparative strengths and weaknesses of these three types of models have recently been reviewed by Watts (1980). Differences between them tend to relate to (1) the number of spatial dimensions along which the climate system is described, (2) the scope of feedback effects included in each type of model, and (3) whether or not the climate system is treated in dynamic terms. of the three types of models, GCMs are clearly the most sophisticated. They can simulate climatic changes on a three-dimensional basis; they incorporate a much wider range of feedback mechanisms than do the others; and they are 
dynamic, capable in some cases of predicting seasonal variations in the climate (Wetherald and Manabe 1981).

Despite the comparative advantages which GCMs have over other types of climate models, their ability to accurately predict the climatic impacts associated with a doubling or a tripling of the concentration of $\mathrm{CO}_{2}$ in the atmosphere remains much in doubt. According to one review of the predictive capabilities of state-of-the-art GCMs, "shortcomings in the treatment of cloud, precipitation, evaporation, ground hydrology, boundary-layer turbulent transport phenomena, orographic effects, wave-energy absorption and reflection in the high atmosphere, as well as truncation errors arising from lack of sufficient resolution combine to produce arge inaccuracies." (National Research Council 1979). Based on these discrepancies, the report concluded: "we do not consider the existing models to be at all reliable in their predictions of regional climatic changes due to changes in $\mathrm{CO}_{2}$ concentration."

In the last several years, the $\mathrm{climate}$ forecasts generated by many GCM experiments have been reviewed by the National Academy of Sciences (1979) and the World Meteorological Organization (1979). While the conclusions of the two panels differed in some respects, the general points of agreement are as follows:

- Global warming is more likely to occur than global cooling; the range of increase in mean global temperatures that can reasonably be expected to occur lie between $+2^{\circ}$ to $+3.5^{\circ} \mathrm{C}$. (GCMs are not designed to predict the time span over which these changes will occur.)

- On average, global warning would appear to intensify the hydrologic cycle by increasing evaporation from the ocean, cloud cover and precipitation.

- The process of heat exchange between the atmosphere and ocean is poorly understood; if the capacity of the deeper oceans to absorb heat is greater than is currently assumed, then the equilibrium warming of the earth's surface and atmosphere in response to elevated $\mathrm{CO}_{2}$ concentrations may occur more slowly than current models have predicted. 
- Global warming will be accompanied by shifts in the geographic and seasonal distributions of temperature, precipitation, evaporation and soil moisture; however, existing models cannot at this time provide reliable predictions of these changes at the regional leve 1 .

Conclusions as general and as guarded as those outlined above do not provide a very solid basis for forecasting the impacts of a $\mathrm{CO}_{2}$-induced climate change on crop yields. Under these types of conditions it might be tempting for scientists to argue that until atmospheric scientists can develop more accurate models with greater geographic resolution, no efforts should be undertaken to try to gauge the effect of a $\mathrm{CO}_{2}$-induced climate change on crop yields. On the other hand, it can also be argued that a major purpose of the $\mathrm{CO}_{2}$ and $\mathrm{Cl}$ imate Research Program is to provide information to decisionmakers in both the public and private sectors that will permit them to take timely actions, either to mitigate the undesirable effects or take advantage of the favorable effects of a $\mathrm{CO}_{2}$-induced climate change. In that case, waiting for better information about future climatic changes may be very costly in terms of the opportunities that are missed to take action in the present based on existing information.

Based on this line of reasoning, a strong argument can be made for developing a range of regional climate scenarios that can, in turn, be used in conjunction with existing crop yield and economic models to try to determine the sensitivity of the agricultural system in the U.S. to a broad range of altered climate states. Such an undertaking would certainly not preclude investments in the technical development of climate models. However, it would unquestionably shed some light on the potential value of this type of research investment, particularly if the "best" and "worse" case economic impacts were not dramatically different.

\subsection{ENVIRONMENTAL EFFECTS OF $\mathrm{CO}_{2}$ ON CROP YIELDS}

The term environmental effects, as used in this report, refers to the broad range of impacts that changes in the atmospheric concentration of $\mathrm{CO}_{2}$ 
may have on the physical and natural environments in which agricultural production takes place. These effects include the direct effects of increases in ambient $\mathrm{CO}_{2}$ on the growth of plants and weeds as a result of increased photosynthetic activity. They also include the indirect effects of $\mathrm{CO}_{2}$ on crop production as a result of changes in meteorological conditions such as cloud cover, temperatures, and precipitation and evaporation. A fairly conventional way of classifying the impact of changes in $\mathrm{CO}_{2}$ on the environment is in terms of first-and second-order effects. First-order effects refer to those $\mathrm{CO}_{2}$-induced environmental changes which directly influence the physiological development of commercial crops. Second-order effects, on the other hand, are related to the impact of increases in ambient $\mathrm{CO}_{2}$ on plant pests, i.e., weeds, insects and diseases, which in turn will affect the conditions under which commercial crops are raised.

The relationship between first- and second-order effects is illustrated in Figure 3.1 by means of a simple path diagram. This diagram represents a much simplified model of agricultural production, focusing specifically on the relationship between the ambient concentration of $\mathrm{CO}_{2}\left(\mathrm{CO}_{2}\right)$, meteorological variables (M), pest abundance (P) and crop yields ( $Y$ ). Two types of first-order effects can be identified in the diagram. First of all, elevated concentrations of $\mathrm{CO}_{2}$ have the potential to increase the rate of dry matter production in commercial crops. This effect is illustrated by the diagonal path $\mathrm{CO}_{2} \rightarrow Y$. Second, increases in the atmospheric concentration of $\mathrm{CO}_{2}$ can be expected to induce climatic changes which will be reflected in changes in temperature, precipitation and cloud cover during the growing season. This effect is illustrated by the path $\mathrm{CO}_{2} \rightarrow M \rightarrow Y$. Second-order effects are somewhat more circuitous, but follow the same general pattern. Changes in ambient $\mathrm{CO}_{2}$ can also be expected to affect photosynthate production in weed pests which will, in turn, intensify the competition between weeds and commercial crops. This effect is indicated by the path $\mathrm{CO}_{2} \rightarrow \mathrm{P} \rightarrow \mathrm{Y}$. Finally, $\mathrm{CO}_{2}$-induced climatic changes have the potential to 


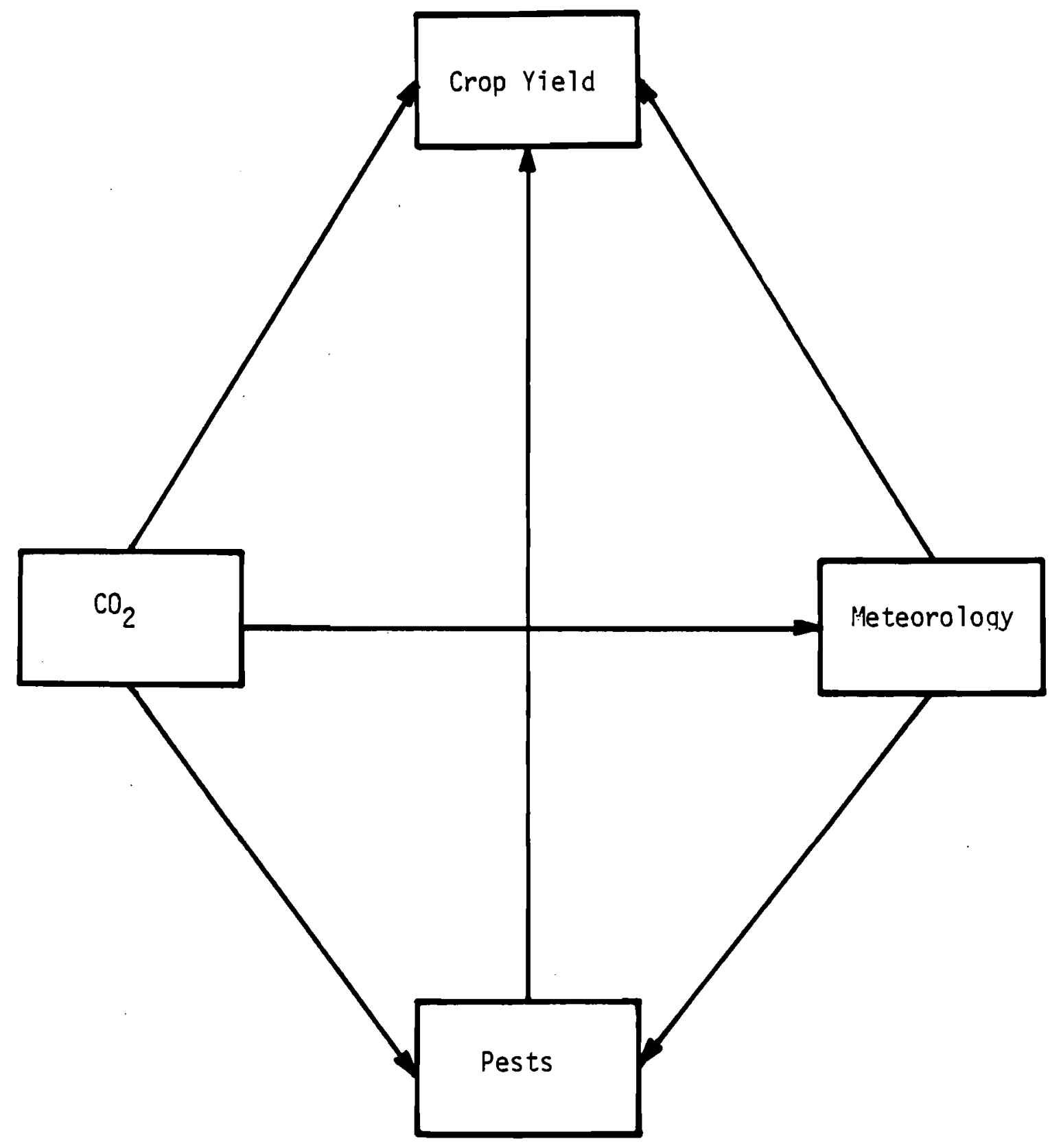

FIGURE 3.1. Path Diagram of Environment-Crop Interactions 
alter the geographic distribution and abundance of a wide variety of pests in ways that may substantially increase crop losses and the costs of pest control. This effect is shown by the path $\mathrm{CO}_{2}+M \rightarrow P \rightarrow Y$.

\subsubsection{First-Order Effects}

of the two types of first-order effects illustrated in Figure 3.1, the one that has received the most attention can be described by the path $\mathrm{CO}_{2} \rightarrow M \rightarrow Y$. While the effects of future increases in ambient $\mathrm{CO}_{2}$ on crop yields could be transmitted through changes in a wide range of climatic conditions, it seems likely that the most important impacts on agricultural production could occur as a direct result of $\mathrm{CO}_{2}$-induced changes in solar radiation, air temperature and precipitation and evaporation--all of which can be regarded as limiting factors on plant growth. Rather than speculate directly about the effects that changes in these variables will have upon the magnitude and geographic distribution of crop yields in the U.S., the purpose of describing them is to provide a very basic understanding of the linkages between crops and the environment.

Solar Radiation

Increases in the concentration of $\mathrm{CO}_{2}$ in the atmosphere have the potential to affect crop yields through changes in cloud cover which alter the amount of radiant energy that strikes the leaves of a plant. The primary effect of solar radiation on the physiological development of plants occurs as a result of photosynthesis. Photosynthesis is a process by means of which an illuminated green leaf converts $\mathrm{CO}_{2}$ into simple sugars. Photosynthetic rates of many commercial crops tend to increase at a constant or decreasing rate with the intensity of solar radiation up to a threshold level, beyond which additional light has little or no effect up on the production of carbohydrates (Gaastra 1959). The maximum light saturation point of most plants usually occurs at intensities below that of full sunlight at noon, although Baker and Hesketh (1967) noted increases in the photosynthetic activity of corn at even higher intensities. The supply of solar radiation available to plants during daylight hours depends not only on cloud cover, but also varies with latitude and the time of year. Yield reductions due to shading also vary widely from crop to crop and with the developmental stage in 
which shading occurs (Evans and Wardlow 1976; Fisher 1975). Thus, while $\mathrm{CO}_{2}$-induced changes in cloud cover may influence crop yields, the effects will be differential, subject to the location and type of crop grown and to the stage of crop growth during which changes in shading occur.

\section{Temperature}

$\mathrm{CO}_{2}$-induced variations in temperature could directly affect a number of the physiological growth processes of plants, including photosynthesis, respiration, soil water uptake, transpiration and vegetative growth. The observed relationship between temperature and growth is nonlinear. For most plants, the rate of growth of plant organs increases from a cardinal minimum temperature of $0^{\circ}$ to $10^{\circ} \mathrm{C}$ up to an optimum range of from $10^{\circ}$ to $35^{\circ} \mathrm{C}$ or more, over which increases in temperature have no affect on rates of growth, and then falls rapidly until a cardinal maximum temperature is reached, beyond which the plant suffers irreversible heat stress (Evans 1975). While the general shape of the temperature curve is remarkably similar for most plants, there is considerable variation in the values of the cardinal temperatures among different species. For example, the range of optimum growth for sugar beets occurs between approximately $20^{\circ}$ to $30^{\circ} \mathrm{C}$, while the corresponding optimum for corn extends from about $25^{\circ}$ to $38^{\circ} \mathrm{C}$ (Fick et al. 1975; de Wit et a 1. 1970). In addition, plant growth in response to increased temperatures will also be influenced by the previous temperatures to which the plant has been exposed, the stage of development during which the increase occurs (Ludlow and Wilson 1971), and perhaps most importantly by the crop water status of the plant (de Wit et al. 1978). Under very dry conditions, transpiration is reduced and this makes the plant more susceptible to the effects of heat stress. Finally, prolonged temperature increases will also affect the length of the growing season, although the increase in frost-free days that occurs as a result of this will depend upon the length of the growing season prior to increased temperatures as we 11 as the distribution of higher temperatures throughout the growing season. (Bollman and Hellyer 1974).

Precipitation and Evaporation

Only a fraction of the water that strikes the soil surface in the form of precipitation or through irrigation is made available to the root zone of a 
plant in the form of soil moisture. The water that is retained in the root zone can be taken up by the plant, along with soil nutrients, and evaporated through the stomata of the leaves by means of the process of transpiration. The water status of a plant during periods of active growth is determined by the balance between transpiration and water uptake from the soil. When soil moisture is not limiting, the rate of transpiration is positively influenced by the flux of radiant energy and temperature. However, when soil moisture is limiting, dehydration of plant leaves (loss of turgor) will cause the stomata to close and this, in turn, will reduce transpiration and photosynthetic activity. At the same time, a larger portion of the carbohydrate reserves in the plant will be made available for root growth to find more water and thus equalize the imbalance between the supply and demand for water. In the process, the vegetative development of the plant will slow down. If the period of stress is long enough, or is accompanied by very high temperatures, the plant will become irreversibly stressed and eventually die if additional soil moisture is not provided to the roots. While no plant is absolutely immune to water stress, the relationship between crop yields and limited soil moisture availability is much less straightforward. Factors that will affect the degree of yield reduction include not only the severity and duration of the water deficit, but also its timing (Salter and Goode 1967; Bauer 1972) the degree of adaptation to water stress exhibited by particular plant species (Boyer and McPherson 1975), the differential impact of the deficit on the part of the plant which is harvested (Fisher and Hagen 1965) and, as mentioned earlier, temperature.

The second type of first-order effect requiring attention involves the potential enhancement of crop yields due to $\mathrm{CO}_{2}$ fertilization. This phenomenon has attracted a great deal of recent attention in the $\mathrm{CO}_{2}$ and climate literature. However, the potential for increasing the dry matter production of commercial crops through $\mathrm{CO}_{2}$ fertilization is hardly a recent finding. More than 175 years ago de Saussure (1804) observed that peas exposed to an atmosphere of $8 \% \mathrm{CO}_{2}$ grew more vigorously than they did under ambient concentrations. Since that time a large volume of scientific literature has grown up around the topic of $\mathrm{CO}_{2}$ enrichment. A comprehensive review of plant responses to elevated $\mathrm{CO}_{2}$ concentrations by Strain (1977) 
uncovered near ly 600 studies reporting increases in photosynthetic rates, vegetative growth and water use efficiencies. In all, 31 different types of responses to increased $\mathrm{CO}_{2}$ were reported, nearly all of which suggest that increasing the concentration of $\mathrm{CO}_{2}$ in the atmosphere will have a positive effect on crop yields. The generally favorable results that have been observed in many past experiments have led Wittwer (1980) to conclude:

The low carbon dioxide concentrations ambient to plant foliage remain the single most important rate determinant for further increases in photosynthes is and crop production. In other words, the currently low level of atmospheric carbon dioxide may well be the most limiting factor in overall global agricultural productivity.

There is certainly room for cautious optimism regarding $\mathrm{CO}_{2}$ enrichment. However, considerably more empirical research needs to be done before it can be concluded that $\mathrm{CO}_{2}$ is indeed the most limiting factor in agricultural productivity. One of the most important questions that has to be resolved relates to the long-term nature of the effects of high $\mathrm{CO}_{2}$ concentrations on $\mathrm{plant}$ growth. Many of the results reported in the literature on $\mathrm{CO}_{2}$ enrichment are based on short-term experiments which do not necessarily provide a reliable basis for determining how plants will respond at different stages in their life cycles. For example, Aoki and Yabuki (1977) found that photosynthetic rates of cucumbers grown under high $\mathrm{CO}_{2}$ concentrations initially rose very rapidly, but then fell below the control rate after just 15 days. Longer-term experiments al so reveal substantial variations in $\mathrm{plant}$ response to high concentrations of $\mathrm{CO}_{2}$ across species (Mauney et al. 1978).

Many of these latter differences can be attributed to the type of carbon fixation pathway that is present in a particular plant species. Plants equipped with a three carbon pathway (C3 plants) exhibit a smaller stomatal response to elevated $\mathrm{CO}_{2}$ concentrations than do plants with a four carbon pathway ( $\mathrm{C} 4$ plants). The ability of $\mathrm{C} 4 \mathrm{plants}$ to close their stomata in the presence of high $\mathrm{CO}_{2}$ concentrations tends to offset the direct effect of $\mathrm{CO}_{2}$ on photosynthesis. While there is still a net gain in the production of carbohydrates by these plants, it is generally not as great as it is for C3 
plants whose stomata are not much affected by $\mathrm{CO}_{2} \cdot$ Other differences in plant response to elevated $\mathrm{CO}_{2}$ concentrations appear to be related to the stage of plant development and whether the plant is a determinant or indeterminant species (Kramer 1981). In general, the largest growth response to $\mathrm{CO}_{2}$ appears to occur in seedlings. It also appears that indeterminant plants, such as cotton and soybeans, respond more vigorously to increased $\mathrm{CO}_{2}$ than do determinant plants such as corn and sorghum.

A second issue that needs to be investigated more thoroughly is the relationship between $\mathrm{CO}_{2}$ and the allocation of $\mathrm{plant}$ reserves to different organs. Many studies have reported atypical morphological patterns as a direct result of exposing plants to high $\mathrm{CO}_{2}$ concentrations. These effects include the accumulation of starch in leaves and stems leading to abnormally thick leaves and greater stem elongation, an increase in branching of both woody and herbacious plants, increased tubor formation and an increase in root/shoot ratios (Kramer 1981; Strain 1978). All of these side effects are related in some way to significant increases in the supply of plant reserves due to the effect of high $\mathrm{CO}_{2}$ concentrations on photosynthesis. However, very dramatic increases in the production of dry matter by plants exposed to high $\mathrm{CO}_{2}$ concentrations by no means imply equally large or even proportional gains in yields. Until scientists find some way to relate changes in the concentration of ambient $\mathrm{CO}_{2}$ to the partitioning of dry matter by plants, the exact nature of the conditions under which crop yields will actually increase cannot be determined.

A final problem relates to observed increases in the water-use efficiency of plants subjected to high concentrations of $\mathrm{CO}_{2}$. When plants are exposed to high $\mathrm{CO}_{2}$ concentrations, transpiration tends to fall as a result of a decrease in the size of the stomatal aperture. At the same time, there is a net gain in photsynthetic activity. Thus, the plant should produce more carbohydrates using less water (Baker et al. 1981). This response has been observed in a number of crops including sorghum (Pallas 1965), corn (Goudrian and van Laar 1978) and wheat (Gifford 1979). Whether this phenomenom can be generalized to all crops is far from clear since there is, as yet, no generally accepted theory of stomatal behavior, nor a sufficiently broad set 
of observations that can be used to compare existing hypotheses. Moreover, the effect of heat stress upon plants in combination with elevated $\mathrm{CO}_{2}$ concentrations is not well documented. Thus, while increases in water-use efficiency may occur, the subsequent reduction in transpiration due to stomatal closure may make plants more vulnerable to heat stress under much warmer and drier conditions.

Overall, then, there is reason for a certain amount of caution in reaching conclusions about the direct effects of high ambient $\mathrm{CO}_{2}$ concentrations on crop yields. It is probably safe to say that the net effect will be positive. However, until a much larger number of long-term experiments, conducted under a variety of meteorological conditions, have been completed, it is impossible to make any reliable predictions about the global effects of anticipated increases in the atmospheric concentration of $\mathrm{CO}_{2}$.

\subsubsection{Second-Order Effects}

According to some estimates, the value of preharvest crop losses due to pest damage in the U.S. is currently running at about 30 to $35 \%$ of the total value of agricultural production (Pimental 1976). There is growing concern within the scientific community that a future $r$ ise in the ambient concentration of $\mathrm{CO}_{2}$ may substantially increase these losses through changes in the abundance and geographic distribution of pests. These changes could occur as a result of $\mathrm{CO}_{2}$-induced changes in agrometeorological conditions (represented by the path $\mathrm{CO}_{2} \rightarrow M+P \rightarrow Y$ in Figure 3.1) or as a direct result of the $\mathrm{CO}_{2}$ enrichment effect upon weed growth (described by the path $\mathrm{CO}_{2} \rightarrow P \rightarrow Y$ ). The former includes pest-specific meteorological effects on weeds, insects, and plant diseases.

\section{Weeds}

Weeds compete with commercially grown crops for solar radiation, soil mo isture, and plant nutrients. Changes in climate which alter the conditions under which commercial crops can be grown profitably can also be expected to affect both the abundance and geographic distribution of weed species. However, because weeds exhibit greater genetic diversity and, thus, are more adaptable to meteorological extremes than crops generally are, small changes 
in climate are likely to cause more dramatic changes in the density and distribution of weeds (Wittwer 1981). The impact of these changes on crop production will be most severe under conditions where soil moisture, temperature, or solar radiation become limiting factors upon crop growth. In these situations, the intensity of competition between crops and weeds for limited resources will be increased, and weed management costs to the farmer are likely to rise (Pimental 1980). Hotter, drier conditions could also increase the competitive edge of $\mathrm{C} 4$ weeds, many of which are particularly noxious, over $\mathrm{C} 3 \mathrm{plants}$ due to the higher water use efficiencies of the former (Black and Chen 1969). These same conditions might further aggravate the competition between weeds and $\mathrm{plants}$ by reducing the uptake of herbicides in weeds.

\section{Insects}

Insects, even more than weeds, are very climate sensitive. Small changes in temperature, in the range of $1^{\circ}$ to $3^{\circ} \mathrm{C}$, can dramatically affect the growth, mobility, and feeding rates of many insect species. According to Pimental, $\mathrm{CO}_{2}$-induced increases in the length of growing seasons in some agricultural regions could lead to the development of from one to three additional generations of insects, depending upon the species (Pimental and Pimental 1978). Just as climate can amplify the impact of insects, so too insects can amplify the effects of climate. For example, in regions that become hotter and drier, crop species that may already be near the limits of their natural range would become more vulnerable to insect damage. $\mathrm{CO}_{2}$-induced climate changes also have the potential to affect climatic release in insect populations. In both the Great Plains and the Canadian wheat belt, severe outbreaks of grasshoppers can be traced directly to a sequence of atypical weather patterns which, over a two- or three-year period, disrupt the natural mechanisms that ordinarily keep the population in check at or near the carrying capacity of the environment (Gage and Mukerji 1977). Insect plagues such as these represent just one example of the many ways in which changes in climate can upset the temporal synchronization between the life cycles of $\mathrm{plants}$ and insects. Changes in climate can also disrupt this synchronization 
through geographic shifts in crop production which expose crops to different insect species, by temperature and/or moisture induced shifts in the range of insect populations, or in combination.

\section{Plant Diseases}

While entomologists and plant pathologists have always been sensitive to climatic influences on the severity and geographic distribution of plant diseases, very little is known about the quantitative aspects of these impacts. On a purely conceptual basis, some of the different kinds of impacts that could be associated with a $\mathrm{CO}_{2}$-induced climate change include:

- the effect of changes in temperature, precipitation and humidity on the growth of various kinds of fungi and on the replication of viruses.

- the effect of altered wind patterns on the dispersal of airborne plant pathogens.

- the indirect effects of climatic change upon the abundance of disease vectors.

- the indirect effects of climatic change upon damage thresholds and the resistence of host plants to combat diseases.

An increase in the ambient concentration of $\mathrm{CO}_{2}$ has the same potential to boost the rate of photosynthetic production in weeds as it does in crops. Practically no research has been done to examine either the short-or long-term effects of high ambient $\mathrm{CO}_{2}$ concentrations on isolated weed species (Strain 1978). However, at least one study has focused on the effect of increases in the atmospheric concentration of $\mathrm{CO}_{2}$ upon the competition between weeds and plants with different carbon fixation pathways (Patterson and Flint 1980). The experimental evidence provided by this study generally supported the hypothes is that $\mathrm{C} 3$ weeds and plants grew more vigorously than C4 weeds and $\mathrm{plants}$ when exposed to high concentrations of ambient $\mathrm{CO}_{2}$. In particular, the study showed that under these conditions velvet leaf ( $C 3$ weed) was more competitive with corn ( $\mathrm{C} 4 \mathrm{crop}$ ) and conversely that soybeans ( $\mathrm{C} 3$ crop) were more competitive with itchgrass ( $C 4$ weed). However, these results 
are fragmentary, and before any general conclusions can be drawn about the direct effects of $\mathrm{CO}_{2}$ on crop-weed competition similar experiments will have to be repeated for a much greater number of crop-weed combinations, under a wide range of environmental conditions.

\subsubsection{Joint Effects}

The discussion of the environmental effects of projected increases in the atmospheric concentration of $\mathrm{CO}_{2}$ on crop yields has thus far dealt with first- and second-order impacts in relative isolation from one another, when in fact both will occur jointly. Consequently, any serious effort to predict the effects of changes in the atmospheric concentration of $\mathrm{CO}_{2}$ on crop yields must also take into account the joint nature of first- and second-order effects. This is implicit in the path diagram shown in Figure 3.1. Referring back to it will help to provide a better understanding of the conceptual issues that are involved in looking at the joint effects of both first-and second-order environmental changes on crop yields.

The relationships illustrated in Figure 3.1 can be written in the following functional form

$$
\begin{aligned}
& Y=f_{1}\left(\mathrm{CO}_{2}, M, P\right) \\
& M=f_{2}\left(\mathrm{CO}_{2}\right) \\
& P=f_{3}\left(M, \mathrm{CO}_{2}\right)
\end{aligned}
$$

where each of the f's simply denote that the magnitude of the left-hand side variable in each equation is some general function of the magnitude of the right-hand side variables. By making the appropriate substitutions, Equations (3.1a) can be condensed into a single yield function

$$
Y=f_{1}\left[\mathrm{CO}_{2}, f_{2}\left(\mathrm{CO}_{2}\right), \mathrm{f}_{3}\left(\mathrm{f}_{2}\left(\mathrm{CO}_{2}\right), \mathrm{CO}_{2}\right)\right]
$$

or more genera $11 y$ 


$$
Y=F\left(\mathrm{CO}_{2}\right)
$$

so that $Y$ is ult imately only a function of a single exogenous variable, $\mathrm{CO}_{2}$. Equations (3.2) represent a very superficial description of the relationship between the yield of a crop and the natural and physical environment. Nevertheless, they can still be used to illustrate that the ultimate effect of a change in the concentration of $\mathrm{CO}_{2}$ in the atmosphere on the yield of crop depends upon a fairly complicated causal chain.

Specifically, what is the effect of a very small increase in the concentration of $\mathrm{CO}_{2}$ on $\mathrm{Y}$ ? The answer to this question can be expressed as the first derivative of yields with respect to $\mathrm{CO}_{2}$ or

$$
\frac{d Y}{d \mathrm{CO}_{2}}=\frac{\mathrm{dF}\left(\mathrm{CO}_{2}\right)}{\mathrm{dCO}_{2}}
$$

which in turn can be written more specifically using (3.2b) as

$$
\frac{d Y}{d \mathrm{CO}_{2}}=\frac{\partial f_{1}}{\partial \mathrm{CO}_{2}}+\frac{\partial f_{1}}{\partial f_{2}} \frac{d f_{2}}{d \mathrm{CO}_{2}}+\frac{\partial f_{1}}{\partial f_{3}} \frac{\partial f_{3}}{\partial f_{2}} \frac{d f_{2}}{d \mathrm{CO}_{2}}+\frac{\partial f_{1}}{\partial f_{3}} \frac{\partial f_{3}}{\partial \mathrm{CO}_{2}}
$$

This rather formidable looking expression can easily be decomposed into the two different sets of first- and second-order effects on crop yields. The first term on the right-hand side of (3.3b) is the direct effect of $\mathrm{CO}_{2}$ on crop yield due to enhanced photosynthesis. The second set of terms represents the effect of a change in $\mathrm{CO}_{2}$ on crop yields due to the intervening effect of $\mathrm{CO}_{2}$ on $\mathrm{climate}$. The third and fourth set of terms represent second-order effects. The third is the effect of pests on yield due to $\mathrm{CO}_{2}$-induced changes in climate, while the fourth and final one expresses the specific effect of weeds on yield due to $\mathrm{CO}_{2}$ enrichment. Thus, each of the expressions in (3.3b) can be related directly back to a specific path in Fiqure 3.1 .

More importantly, it is clear from (3.3b) that the magnitude and even the direction of the impact of a small increase in $\mathrm{CO}_{2}$ on crop yield cannot be 
determined a prior $i$ without specific knowledge of all of the functional relationships originally expressed in Equation (3.1). Again, this does not mean that researchers must have perfect information about all of these relationships before they can elther forecast or construct scenarios based upon the effects of changes in $\mathrm{CO}_{2}$ on crop yields. However, it does suggest that efforts in either direction which proceed on the bas is of partial information about the relationships expressed in (3.3b) must do so with a clear understanding of the consequences of assigning too large or too small a weight to any of the unknown effects.

\subsection{DIRECT ECONOMIC IMPACTS OF CO 2 -INDUCED ENVIRONMENTAL CHANGES}

Direct economic impacts refer to the effects of $\mathrm{CO}_{2}$-induced environmental changes on the production of raw agricultural commodities which occur in one of two ways. First of all, $\mathrm{CO}_{2}$-1nduced changes in the environment have the potential to alter the total quantities of different agricultural commodities produced by a single firm. These output changes can occur either as a direct result of the effect of the environment upon crop yields, or as an indirect result of changes in the environment upon the mix of inputs used by the firm to produce different agricultural commodities. Either of these two types of changes will exert some influence on the cost of increasing the output of one or more agricultural commodities, causing the firm to produce these goods at different levels of output than it would otherwise have selected.

A second way that $\mathrm{CO}_{2}$-induced environmental changes could directly affect the production of agricultural commodities is through changes in the market prices of these goods. Most agricultural commodity markets are highly competitive: no single firm can significantly influence the prices that prevail in these markets. As such, the response of a single firm to $\mathrm{CO}_{2}$-induced changes in the environment will not affect the market prices of the commodities which it produces. However, wide-spread changes in the environment, which affect many different producers, will also influence the relationship between marginal cost and output embodied in the supply functions of a large number of agricultural goods. Consequently, $\mathrm{CO}_{2}$-induced shifts 
in the market supply function for any commodity will cause a change in the equilibrium price and level of output associated with that good. This will, in turn, affect the behavior of individual producers who would have to factor these different prices into their production decisions. In concert, the two types of direct impacts outlined above will generally involve changes in: (1) average yields, (2) utilization of inputs to production, including the distribution of cropland and (3) farm income.

The remainder of this secion will be used to develop a systematic framework designed to explain how these changes can occur, first at the level of the individual firm and then in terms of market behavior. The treatment of economic phenomena in Sections 3.3.1 to 3.4.2 is somewhat technical. Therefore, the reader who may not want to follow the mathematical development of economic relationships may wish to turn to Section 3.5 , which contains a more straightforward sumary of the analysis.

\subsubsection{Resource Allocation by the Firm}

The technology of a multi-output, multi-input firm, characterized by non-jointness in outputs and variable inputs (Lau 1978; Shumway et al. 1981) can be expressed by

$$
Q_{i}=f_{i}\left(x_{i 1}, \ldots, x_{i n}, z_{i 1}, \ldots, z_{i k}, \bar{E}\right) \quad \text { for all } i
$$

and

$$
\sum_{i=1}^{m} z_{i h}=\bar{z}_{h} \quad \text { for all } h
$$

where $i=1, \ldots, m$ denotes the different commodities produced by the firm; $j=1, \ldots, n$ and $h=1, \ldots, k$ denote the variable inputs and allocatable fixed factors used by the firm, respectively, and

$$
\begin{aligned}
Q_{i}= & \text { the quantity of the } i \text { th commodity produced by the firm } \\
x_{i j}= & \text { the quantity of the } j \text { th variable input used to produce the } \\
& i \text { th commodity }
\end{aligned}
$$


$z_{\text {in }}=$ the quantity of the hth allocatable fixed factor used to produce the ith commodity

$\bar{z}_{h}=$ the quantity of the hth fixed factor owned by the firm

$\bar{E}=a$ vector indicating the magnitudes of environmental variables

likely to be influenced by changes in the atmospheric concentration of $\mathrm{CO}_{2}$.

The function $f_{j}()$ is commonly referred to as a production function. It expresses the maximum output of the ith commodity that can be obtained by combining both variable inputs and allocatable fixed factors, given the state of the environment as characterized by $\bar{E}$. Production functions are typically assumed to be single-valued continuous functions with continuous, non-decreasing first-order partial derivatives in both $x_{i j}$ and $z_{i h}$. Second-order derivatives are traditionally restricted as follows:

$$
\frac{\partial^{2} Q_{i}}{\partial x_{i j}^{2}} \leq 0 \quad \frac{\partial^{2} Q_{i}}{\partial z_{i h}^{2}} \leq 0
$$

to ensure diminishing marginal productivity in both variable and fixed inputs. No restrictions are imposed, a priori, on the influence of environmental variables: changes in $\bar{E}$ will either increase or decrease output, depending upon the observed relationship between $Q_{j}$ and $\bar{E}$.

Equation (3.4b) represents a set of $k$ constraints on the technology of the firm due to the presence of allocatable fixed factors. Allocatable fixed factors are divisible in the sense that each unit of one of these inputs can be distinguished on the basis of the production process in which it is used. The most common type of allocatable fixed factor employed in agriculture is land. Other examples include family labor and the capital services associated with many different kinds of farm machinery and implements. Equation (3.4b) constrains the total amount of each allocatable fixed factor that can be used by the firm to the quantity which it owns in the current period. 
There are, literally, an infinite number of variable and fixed input combinations which can satisfy the relationships set forth in Equations (3.4). As such, these equations do not constitute a model of economic behavior, nor do they shed a great deal of light on the effect of $\mathrm{CO}_{2}$-induced changes in the vector $\bar{E}$ upon the behavior of the firm. In order to do either of these things, the technology of the firm must be set in the framework of a specific decision-making problem, such that choice of inputs and outputs is consistent with a particular objective of the firm.

A fundamental postulate of neoclassical production theory is that economic agents, in this case agricultural producers, behave rationally. This means that the decisions made within a firm can be viewed in terms of the pursuit of a specific economic objective. While many different objectives are consistent with economic rationality on the part of the firm, it will be assumed throughout the remainder of this report that the behavior of agricultural producers can be characterized specifically in terms of the objective of profit maximization. In the case of a multi-output, multi-input firm whose technology is characterized by (3.4), the resource allocation problem that is consistent with this objective can be formulated as

Maximize $\Pi=\sum_{i=1}^{m} P_{i} Q_{i}-\sum_{i=1}^{m} C_{i}^{*}\left(Q_{i}, r_{1}, \ldots, r_{n}, z_{i 1}, \ldots, z_{i k}, \bar{E}\right)-F C$

subject to

$$
\sum_{i=1}^{m} z_{i h}=\bar{z}_{h} \quad \text { for all } h
$$

where

$$
\begin{aligned}
\Pi & =\text { the profits of the firm } \\
P_{i} & =\text { the constant, per unit price of the ith commodity } \\
r_{j} & =\text { the constant, per unit price of the jth variable input } \\
F C & =\text { the fixed cost of the firm }
\end{aligned}
$$

The variable cost (VC) function 


$$
\sum_{i}^{m} c_{i}^{*}\left(Q_{i}, r_{1}, \ldots, r_{n}, z_{i 1}, \ldots, z_{i k}, \bar{E}\right)
$$

is obtained as the solution to the problem

$$
v C=\sum_{j=1}^{n} r_{j} \sum_{i=1}^{m} x_{i j}
$$

subject to the production functions in (3.4a). The additivity of the variable cost function is a direct result of non-jointness in outputs and variable inputs (Lau 1978). Consequently, each of the additive components in the variable cost function represents the minimum cost to the firm of producing $Q_{i}$ units of the ith commodity using both variable inputs purchased at constant unit prices $r_{1}, \ldots, r_{n}$ and an arbitrary vector of allocatable fixed factors $z_{i 1}, \ldots, z_{i k}$, given the state of the environment implied by $\bar{E}$.

The maximization problem expressed in Equation (3.5) is fairly general in the sense that it can be used to characterize the behavior of profit maximizing firms with a variety of different technologies, under a fairly broad range of economic conditions. However, there are several areas in which this model either imposes restrictions on the technology of the firm which may not always be justified, or is based on simplifying assumptions about the nature of the economic environment. While it is always difficult to strike a proper balance between the general and the particular in presenting an analytical model of this sort, a brief list of its shortcomings will provide a useful introduction to some of the problems encountered in constructing models for applied use.

Restrictions on Technology. Nonjointness in outputs and variable inputs is a useful assumption that makes it possible to isolate the effect of $\mathrm{CO}_{2}$-induced environmental changes on the production of different agricultural comodities. However, imposing this restriction rules out the existence of interdependencies between the production of commodities, a characieristic that is not uncomon in the agricultural sector. 
Intertemporal Problems. The characterization of technology and the structure of the profit maximization model in Equation (3.5) imply that all production decisions are made at a single point in time. In fact, agricultural production is by its very nature a dynamic process, such that the timing of production decisions throughout the growing season may have a profound impact on the profits of the firm.

Treatment of Output Prices. The model contains no distinction between the decisions which producers make based on the prices they expect to receive as opposed to the prices they actually receive when commodities are marketed. As such, it ignores decisions that are based on periodic revisions of price expectations by producers as the growing season progresses.

Treatment of Environmental Variables. The state of the environment does not remain constant over the growing season. At any given time, producers have expectations regarding future states of the environment, but they rarely know with absolute certainty how meteorological conditions will actually change in the future. The model assumes that producers have perfect information regarding future environmental states, while from a planning standpoint the vector $\bar{E}$ should be treated as random.

From (3.5) one can obtain the following first order conditions

$$
\begin{array}{ll}
\frac{\partial \Pi}{\partial Q_{i}}=P_{i}-\frac{\partial C_{i}^{*}}{\partial Q_{i}}=0 & \text { for all } i \\
\frac{\partial \Pi}{\partial Z_{i h}}=-\frac{\partial C_{i}^{*}}{\partial Z_{i h}}-\lambda_{h}=0 & \text { for all } i \text { and } h \\
\frac{\partial I}{\partial \lambda_{h}}=\bar{Z}_{h}-\sum_{i=1}^{m} Z_{i h}=0 & \text { for all } h
\end{array}
$$

These conditions constitute a set of decision rules governing the production of $Q_{i}$ and the use of allocatable fixed factors. The first condition requires the firm to increase its production of the ith commodity, until the 
marginal cost of the last unit produced is equal to its market price. The second condition requires the firm to allocate the hth allocatable fixed factor to the production of the ith commodity until the marginal cost of the last unit so employed is equal to the shadow price of that factor, $\lambda_{h}$. The shadow price of a fixed factor represents the additional revenue the firm would earn if it were able to obtain another unit of that input. As such, $\lambda_{h}$ must be non-negative or else the factor with which it is associated would not be used at all. By the same logic $\partial C_{i} / \partial Z_{i h}$ will be non-positive: increasing $Z_{i n}$ must reduce the variable cost of producing $Q_{i}$, otherwise none of the factor would be allocated to that commodity. The last condition simply states that the firm cannot allocate more units of the hth fixed factor than it currently has at its disposal.

An additional piece of information that can be extracted from the original problem is due to the derivative property of cost functions (McFadden 1978; Shephard 1953, 1970). By differentiating each of the variable cost functions in $(3.5 a)$ with respect to the vector of variable input prices $r_{1}$, $\ldots, r_{n}$ one obtains the output-compensated demand functions for the variable inputs used to produce the ith commodity (conditional in this case upon the arbitrary values of $Z_{i n}$ used to derive the minimum variable cost of producing each commodity). These demand functions can be written

$$
x_{i j}=x_{i j}\left(Q_{i}, r_{1}, \ldots, r_{n}, z_{i l}, \ldots, z_{i k}, \bar{E}\right) \quad \text { for } a 11 i \text { and } j
$$

The first order conditions in (3.6) can also be expressed in implicit functional form as

$$
\begin{aligned}
& P_{i}-P_{i}\left(Q_{i}, r_{1}, \ldots, r_{n}, z_{i 1}, \ldots, z_{i k}, \bar{E}\right)=0 \text { for all } i \\
& \lambda_{h}-\lambda_{i h}\left(Q_{i}, r_{1}, \ldots, r_{n}, z_{i 1}, \ldots, z_{i k}, \bar{E}\right)=0 \text { for all } i \text { and } h(3.7 c) \\
& \bar{z}_{h}-\sum_{i=1}^{m} z_{i h}=0 \quad \text { for a } 11 \mathrm{~h}
\end{aligned}
$$


In all, (3.7) contains $m(n+k+1)+k$ equations. Assuming that (3.7)

satisfies the "implicit function" rule, this system of equations can be solved to obtain the optimal values of the corresponding number of decision variables and shadow prices in the model. In general, the optimal values of the observable decision variables $Q_{j}, X_{i j}$, and $Z_{i n}$ can be expressed as functions of the exogenous variables in the model, $P_{i}, r_{j}, z_{h}$ and $\bar{E}$. These functions can be written in vector notation as

$$
\begin{array}{ll}
x_{i j}=X_{i j}^{\star}(P, r, \bar{Z}, \bar{E}) & \text { for all } i \text { and } j \\
Z_{i h}=z_{i h}^{\star}(P, r, \bar{Z}, \bar{E}) & \text { for all } i \text { and } h \\
Q_{i}=Q_{j}^{\star}(P, r, \bar{Z}, \bar{E}) & \text { for all } i
\end{array}
$$

where $P=\left(P_{1}, \ldots, P_{m}\right)$ and $r=\left(r_{1}, \ldots, r_{n}\right)$ are vectors of output and variable input prices, respectively, and $z=\left(z_{1}, \ldots, z_{k}\right)$ represents a vector of fixed factors.

Equations (3.8a) and (3.8b) represent the factor demand equations for the variable and allocatable fixed factors over which the firm has direct control. The relationship between $r_{j}$ and $x_{i j}$ expressed in the former is illustrated by the factor demand curve $D D^{\prime}$ in Figure 3.2. This curve is downward sloping consistent with diminishing marginal productivity of each variable input. Points along the curve represent the contribution of successive units of the input, $x_{i j}$, to the revenue of the firm obtained from the production of $Q_{i}$.

Changes in the price of an input cause the firm to adjust its output along the factor demand curve until the marginal revenue obtained from the use of the last unit of $x_{i j}$ is equal to its market price. This equilibrium occurs at point $M$ in Figure 3.2 where the firm employs $x_{i j}^{\star}$ units of the factor. The total willingness of the producer to pay for this amount of the input, rather than do without it, is given by the integral 


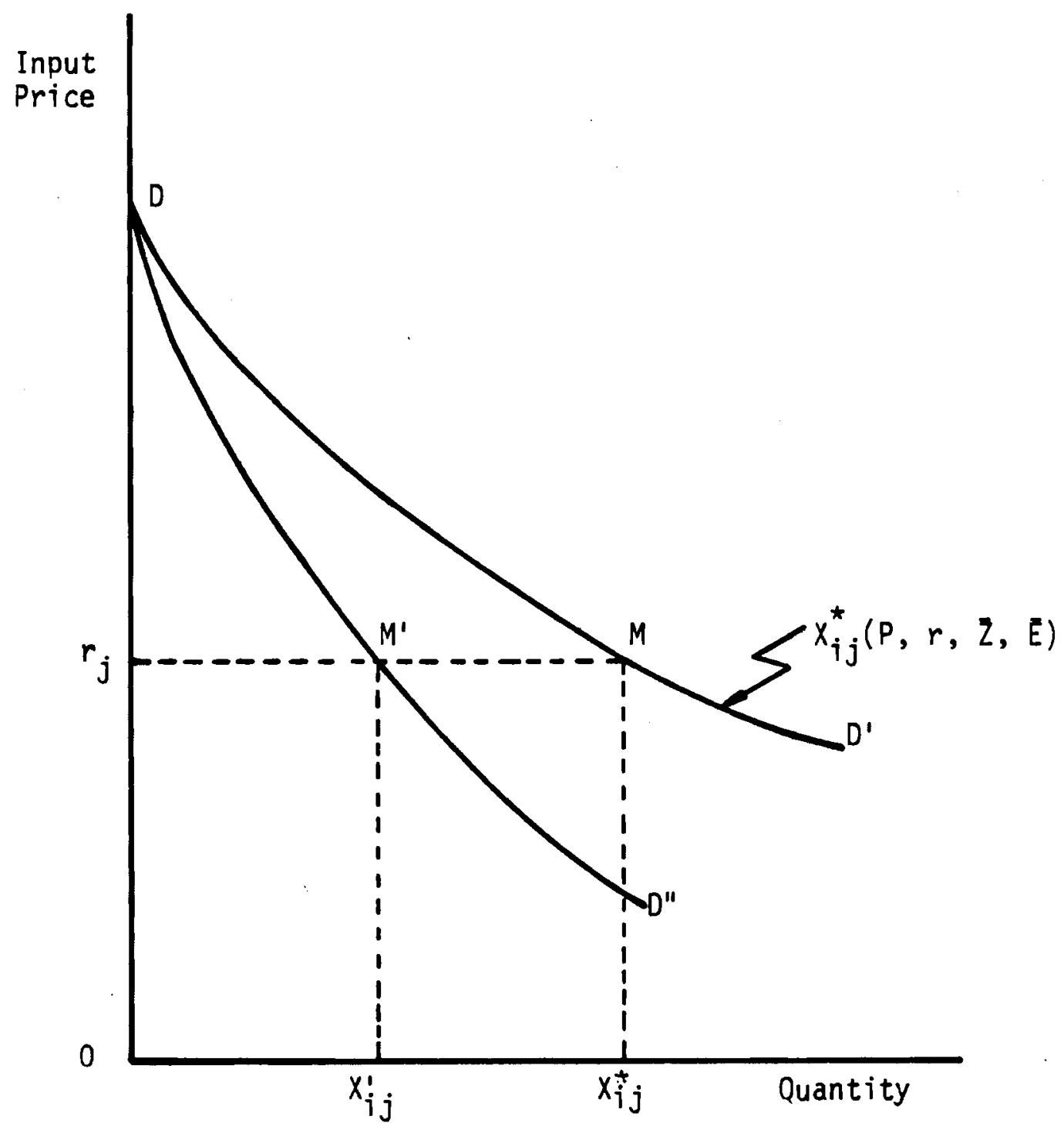

FIGURE 3.2. The Firm's Demand for a Variable Input

$$
\int_{0}^{x_{i j}^{*}} r_{j}\left(P, r, \bar{z}, \bar{E}, x_{i j}\right) d x_{i j}
$$

which is equal to the area $\mathrm{DMX}_{i j}^{*} 0$. The cost of purchasing this input is equal to $r_{j} \cdot x_{i j}^{*}$ which is represented by the area $r_{j} M X_{i j}^{*} 0$. The difference between the total willingness of the firm to pay for the input (rather than go without it) and its total cost, or the area $r_{j} D M$, represents a benefit that accrues 
to the firm as a result of being able to purchase $x_{i j}^{*}$ units of the input at $r_{j}$ dollars when it would have been willing to pay as much as D dollars to use a single unit of the input.

$\mathrm{CO}_{2}$-induced environmental changes will affect the demand for a variable input by shifting the factor demand curve to the right or left, depending on the sign of $\partial x_{i j} / \partial E$. One possible case is $i l l u s t r a t e d$ in Figure 3.2 by a shift in the factor demand to the position DD". In this situation, the firm now uses less of the variable input $\left(x_{i j}^{\prime}<x_{i j}^{*}\right)$. However, the effect that this change will have upon the production of the ith commodity cannot in general be derived from this model since changes in the environment are likely to cause producers to make adjustments in the usage of other variable inputs and allocatable fixed factors, as well. Nor as a general rule will it even be possible to make systematic statements about the direction of change implied by $\partial x_{i j} / \partial E$ without more explicit knowledge of the structure of the individual production functions and the values of both the parameters and the exogenous variables in the factor demand equations represented by (3.8a).

Turning next to (3.8b) which governs the optimal values of the allocatable components of each fixed factor, still less can be said about the influence of changes in $\bar{E}$ upon the individual $Z_{\text {in }}$. Here, the only refutable proposition that emerges regarding the effect of the environment on the allocatable fixed factors is that for each $\bar{z}_{h}$

$$
\sum_{i=1}^{m} \frac{\partial Z_{i h}^{\star}}{\partial E}=0
$$

In other words, the effect of changes in the environment upon the individual components of an allocatable fixed factor must balance out because the endowment of $\bar{Z}_{h}$ is, by definition, fixed. For example, an increase in $\mathrm{CO}_{2}$ which directly or indirectly causes the firm to increase the acreage it uses to produce one crop must lead to decreases in the amount of 1 and allocated to remaining crops. No further refutable propositions emerge from the model regarding the effect of changes in commodity or variable input prices, the 
endowment of fixed factors, or the state of the environment upon the individual $Z_{i h}$ in the model.

Equation (3.8c) represents the supply function of the firm. The relationship between $P_{i}$ and $Q_{j}$ embodied in (3.8c) is indicated by the supply curve OS in Figure 3.3. As drawn, $Q_{i}$ is always increasing in $P_{j}$ : an increase in the price of a commodity will always cause the firm to produce more of it, although the marginal cost of doing so, or $\partial \mathrm{P}_{j} / \partial Q_{j}$, will also increase. Typically, there is a range of output over which the marginal cost of producing $Q_{j}$ at first decreases. However, it can be shown that, under

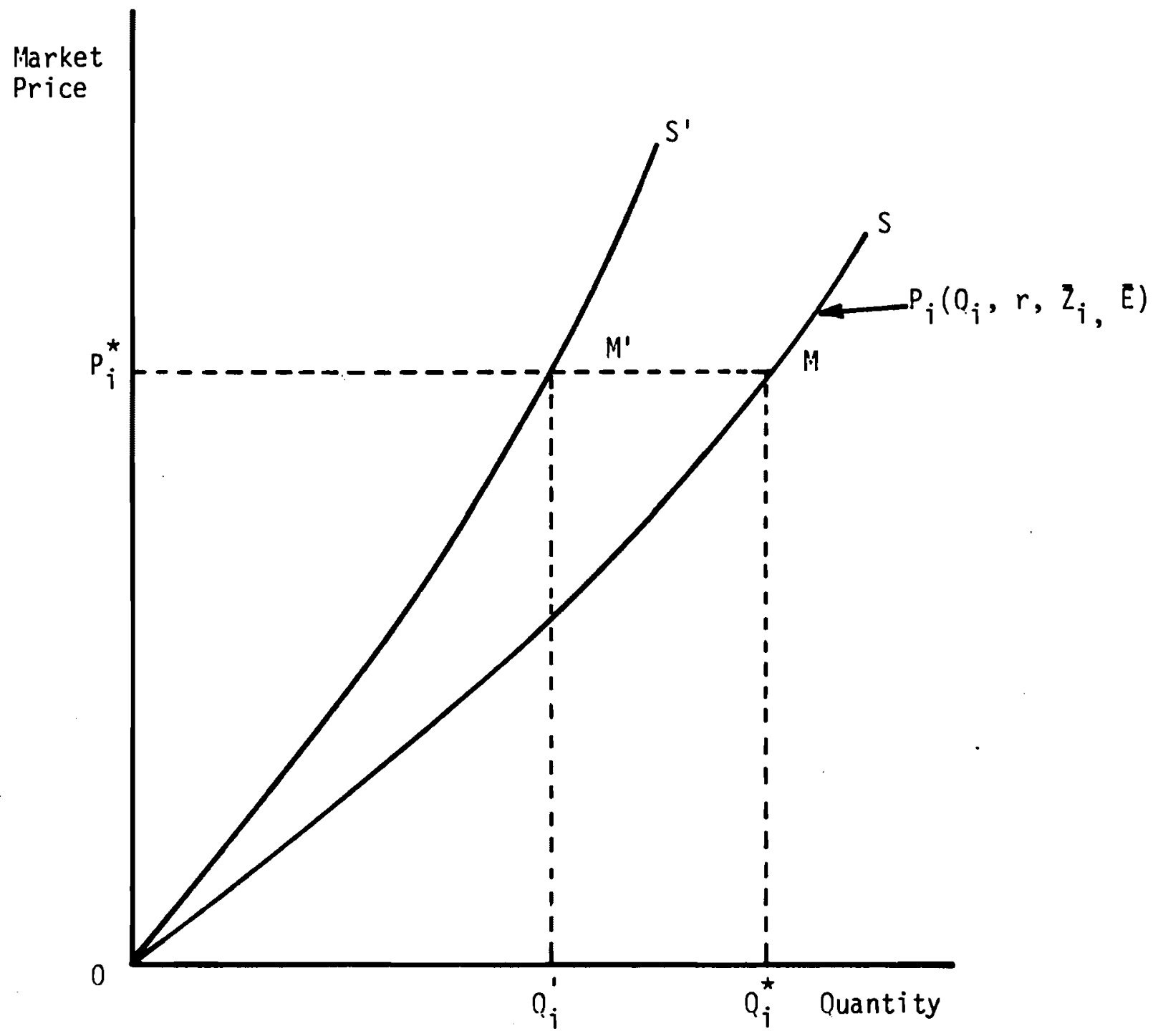

FIGURE 3.3. The Determination of the Optimal Output of the Firm 
competitive conditions, the firm will always select a level of production for which the marginal cost is non-decreasing. Changes in the price of output cause the firm to move along its supply curve according to (3.6a) until the marginal cost of the last unit of $Q_{j}$ produced equals the market price. This occurs at point $M$ on the supply curve, corresponding to the output level $Q_{j}^{\star}$. The revenues obtained from this level of production, or $P_{i}^{*} Q_{j}^{*}$, are represented by the area $P_{j}^{\star} M Q_{j}^{\star} 0$. The integral

$$
\int_{0}^{P_{i}^{*}} Q_{i}(P, r, \bar{Z}, \bar{E}) d P_{i}
$$

represented by the area above the supply curve and below the price line, or $P_{i}^{*} M O$, is the contribution of the $i_{i n}$ commodity to the profits of of the firm. The difference between these two areas represented by $O M_{i}^{*}$ is equal to the variable cost to the firm associated with producing the ith commodity. This is equivalent to the integral

$$
\int_{0}^{Q_{i}^{*}} P_{i}\left(Q_{i}, P, r, \bar{Z}, \bar{E}\right) d Q_{i}
$$

$\mathrm{CO}_{2}$-induced changes in $\bar{E}$ will have the effect of shifting the supply curve to the right or left depending on the direction of $\partial Q_{j} / \partial E$. For example, in a situation where $\mathrm{CO}_{2}$-induced environmental changes adversely impact output, the supply curve illustrated in Figure 3.3 would shift to the left to a position OS'. Assuming no change in price the firm would reduce its production to $Q_{j}^{\prime}$; the revenues it obtains from producing $Q_{j}^{\prime}$ would fall to $P_{j}^{*} M^{\prime} Q_{j}^{\prime} 0$ and $i$ ts variable profits (exclusive of fixed costs) would decrease to $O P_{i}^{*} M^{\prime} Q_{j}^{\prime}$. Exogenous changes in $\bar{E}$ would also affect the production of other commodities depending upon the relationship implied by $\partial Q_{j} / \partial E$ for the remaining goods.

The effect of $\mathrm{CO}_{2}$-induced changes in $\bar{E}$ on the commodity outputs of the firm cannot in general be determined by inspecting the relationship between 
$Q_{j}$ and $\bar{E}$ in the production functions of this firm. To see why it is generally not true that $\partial Q_{j} / \partial E=\partial f_{j} / \partial E$ one can substitute the functional relationships governing the optimal values of $X_{i j}$ and $z_{i h}$ from (3.8a) and $3.8 b$ ) into the production functions shown in (3.4a). The resulting supply function for $Q_{j}$ can then be expressed in vector notation

$$
Q_{i}=f_{i}\left(X_{i}^{*}(P, r, \bar{Z}, \bar{E}), z_{i}^{*}(P, r, \bar{Z}, \bar{E}), \bar{E}\right)
$$

where $x_{i}=\left(x_{i 1}, \ldots, x_{i n}\right)$ and $z_{i}=\left(z_{i 1}, \ldots, z_{i k}\right)$ are vectors representing the quantities of variable and fixed inputs used to produce the ith commodity. Taking the partial derivative of $Q_{j}$ with respect to $\bar{E}$ gives

$$
\frac{\partial Q_{i}}{\partial E}=\sum_{j=1}^{n} \frac{\partial f_{i}}{\partial x_{i j}^{\star}} \frac{\partial x_{i j}^{\star}}{\partial E}+\sum_{h=1}^{k} \frac{\partial f_{i}}{\partial Z_{i h}^{\star}} \frac{\partial Z_{i h}^{\star}}{\partial E}+\frac{\partial f_{i}}{\partial E}
$$

Due to the regularity conditions imposed upon the production function, the signs of the partial derivatives $\partial f_{\mathfrak{i}} / \partial x_{i j}$ and $\partial f_{\mathfrak{i}} / \partial Z_{i h}$ must be nonnegative since a firm will not choose to use an input that decreases its output. In addition, the sign of the partial derivative $\partial f_{j} / \partial E$ may also be known. However, the signs of the partial derivatives $\partial x_{i j} / \partial E$ and $\partial z_{i h} / \partial E$ cannot be determined a priori on the basis of theory unless all of the parameters of the production technology, as well as the values for the exogenous variables in the model, are somehow known in advance.

\subsubsection{Market Supply and Demand}

The results obtained so far relate only to the behavior of a single profit maximizing firm. Under competitive conditions, the output of each firm is assumed to be so small relative to the total output of all firms that changes in the level of output by any one firm will have only an insignificant 
effect on the corresponding market price. Therefore, in the previous section it was implicitly assumed that $\mathrm{CO}_{2}$-induced changes in the environment would have no direct effect on output prices through the behavior of the firm. At the market level, however, it is not unreasonable to expect that changes in the atmospheric concentration of $\mathrm{CO}_{2}$ will affect the production of a large number of firms producing many different kinds of agricultural commodities. In that case, one may also expect changes to occur in the equilibrium, market clearing prices and output levels in a number of different markets.

The mechanism by means of which market prices are determined can be examined by again appealing to a very simple analytical model. In this instance, two assumptions will be made to simplify the analysis. The first is that all of the commodity markets represented in the model are composed of profit maximizing firms, none of which can individually influence market prices in any substantial manner. The second assumption is that the collective actions of all producers will have no effect on input prices. This last assumption is very strong and represents a concession to partial equilibrium analysis, that cannot be justified on empirical grounds. However, it is traditionally employed in examining market price determination because it simplifies the analysis. It will later be modified in the subsequent section dealing with secondary impacts.

An aggregate model of multi-commodity market behavior consistent with these assumptions can be expressed in vector notation as

$$
\operatorname{Maximize} W=\oint_{0}^{Q^{\star}}\left\{\sum_{i=1}^{m} P_{i}(Q, \bar{H}) d Q_{i}\right\}-C^{\star}(Q, r, \bar{Z}, \bar{E})
$$

While certain features of (3.10) are recognizable from (3.5a), the objective function is quite a bit different. The term $W$ is shorthand for a specific measure of welfare that consists of the sum of consumer and producer surplus. Consumer surplus can be defined as the difference between the total amount of money consumers would be prepared to pay for a given quantity of some good and the amount they actually pay for it. Producer surplus is an 
analogous measure which represents the excess of total revenues obtained from the production and sale of a given quantity of some good over the total cost of supplying the good. As such, it is a measure of the profit obtained from a single good. The use of consumer and produce surplus as a measure of welfare was first proposed by Dupuit (1844) and later developed by Marshall (1890). Hotelling $(1932,1938)$ extended the concept and demonstrated that the sum of these two welfare measures was consistent with competitive market behavior on the part of individual producers.

These two measures are illustrated for a single good in Figure 3.4. Here the aggregate demand curve for the commodity $Q$ is indicated by DD', while the corresponding aggregate supply curve, obtained by summing the quantities of that good supplied by separate firms over all prices, is represented by the curve OS. The market supply-demand equilibrium occurs at the point of intersection between the aggregate supply and demand curves. At that point, $Q^{\star}$ is produced at price $P^{\star}$. Consumer surplus, as defined above, is represented by the area under the demand curve and above the market price, or $P^{\star} D M$. This is equivalent to the integral

$$
\int_{0}^{Q^{\star}} P(Q) d Q-P^{\star} Q^{\star}
$$

where $P(Q)$ represents the indirect demand function; the expression to the left of the minus sign is the total willingness to consumers to pay for $Q^{\star}$, or the area $D M Q^{\star} 0$; and $P^{\star} Q^{\star}$ is the total amount of money expended by consumers on $Q^{\star}$.

The total cost of all firms in the market is equal to the area under the supply curve and to the left of $Q^{\star}$, or the area $O M Q^{\star}$. This is equivalent to the integral

$$
C=\int_{0}^{Q^{\star}} C^{\prime}(Q) d Q
$$




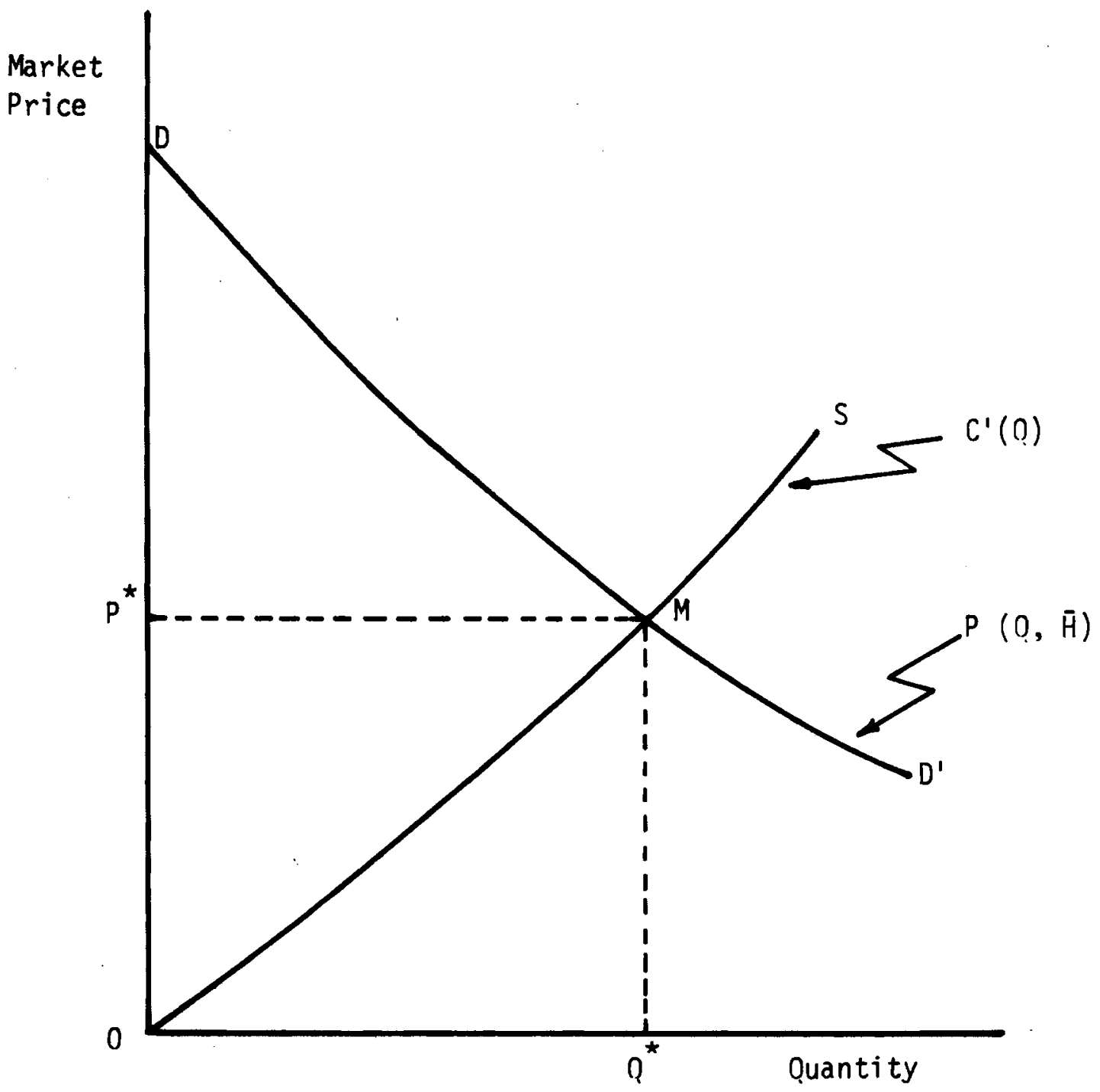

FIGURE 3.4. Determination of Market Price and Quantity Equilibrium

where $C^{\prime}(Q)$ represents the marginal cost, or inverse market supply. function. The area $\mathrm{P}^{\star} \mathrm{MO}$ is equal to producer surplus or the total profits earned in the market from the sale of $Q^{\star}$. This area is represented by the expression

$$
\Pi=\int_{0}^{P^{\star}} Q(P) d P=P^{\star} Q^{\star}-\int_{0}^{Q^{\star}} C^{\prime}(Q) d Q
$$


where $Q(P)$ is the market supply function. In that case one can make the appropriate substitutions to show that the welf are function

$$
W=\int_{0}^{Q^{\star}} P(Q) d Q-\int_{0}^{Q^{\star}} C^{\prime}(Q) d Q
$$

is equivalent to

$$
W=\int_{0}^{Q^{\star}} P(Q) d Q-P^{\star} 0^{\star}+\int_{0}^{P^{\star}} Q(P) d P
$$

which is the sum of producer and consumer surplus shown in Figure 3.4 by the area DMO.

Figure 3.4 represents a very simple case in that the area under the demand curve depends on the price of only one commodity. In general, of course, one would expect the aggregate quantity demanded of a good to be a function of its own price, the prices of other substitute or complementary goods, and a vector of demographic variables including per capita income. In this case, Hotelling (1932) has suggested that the appropriate analogue to the integral used in the preceding example, or

$$
\int_{0}^{Q^{\star}} P(Q) d Q
$$

should be the line integral which appears in the objective function of (3.10) as

$$
\oint_{0}^{Q^{\star}}\left\{\sum_{i=1}^{m} \cdot P_{i}(Q, \bar{H}) d Q_{i}\right\}
$$


where $P_{j}(Q, H)$ represents the indirect demand function for the ith commodity, $Q=\left(Q_{j}, \ldots, Q_{m}\right)$ is a vector of commodity demands and $\bar{H}$ is a vector of exogenous variables that is purposely left vague such that the indirect demand functions may characterize both the final demands of consumers and the intermediate, or derived demands for agricultural commodities from food processors, livestock feeding, exporters and other intermediate uses for these goods. The integration sign in (3.12) simply indicates that the total willingness to pay on the part of consumers for $Q^{*}$ is a line integral along some curve $F(0, Q)$ connecting the origin of $n$-space to the vector $Q$.

The indirect demand function is not familiar to many economists and requires further $\mathrm{clarification.} \mathrm{In} \mathrm{the} \mathrm{case} \mathrm{of} \mathrm{consumers,} \mathrm{the} \mathrm{direct} \mathrm{demand}$ functions associated with food $(i=1, \ldots, m)$ and other goods and services $(m+1, \ldots, t)$ are

$$
Q_{i}=Q_{i}\left(P_{1}, \ldots, P_{t}, \bar{M}\right) \quad \text { for all } i
$$

These demands are derived as the solution to the consumer decision problem

$$
\begin{aligned}
& \text { Maximize } U=U\left(Q_{1}, \ldots, Q_{t}\right) \\
& \text { subject to } \\
& \sum_{i=1}^{t} P_{i} Q_{i}=\bar{M}
\end{aligned}
$$

where $U$ is utility and $\bar{M}$ is consumer income. The indirect demand functions

$$
P_{i}=P_{i}\left(Q_{1}, \ldots, Q_{t}, \bar{M}\right) \quad \text { for all } i
$$

are obtained by inverting the system of direct demand equations, such that prices depend on consumption and income, whereas in the direct demand functions consumption depends on prices and income. 
In the case of the firm which uses agricultural commodities to produce other goods and services, the indirect demand function represents the first derivative of the profit function with respect to the demand for the commodity. Thus, if the profit function of the intermediate firm is

$$
I=P_{0} f_{0}(X, Q)-r X-P Q
$$

then the indirect demand (or marginal value product) function for $Q$ is obtained as

$$
\frac{\partial \Pi}{\partial Q}=P_{0} \frac{\partial f_{0}}{\partial Q}=P=P\left(Q, P_{0}, r\right)
$$

Thus, the characterization of the indirect demand functions in Equation (3.12) as

$$
P_{i}=P_{i}(Q, \bar{H})
$$

is a generalization for the aggregate indirect demand functions associated with both consumer and derived demands for agricultural commodities. In that framework, the vector $\bar{H}$ may include per capita income, the market prices of goods and services that are produced using agricultural commodities and the prices of the resources used to produce these commodities.

The second term in the objective function is the variable cost function for the hypothetical agricultural sector being modeled. It represents the minimum cost in all agricultural markets of producing a vector of commodity outputs $Q=\left(Q_{1}, \ldots, Q_{m}\right)$ using variable inputs purchased at fixed input prices $r=\left(r_{1}, \ldots, r_{n}\right)$ and fixed factors $z=\left(z_{1}, \ldots, z_{k}\right)$, given the state of the the environment as $\bar{E}$. The cost function expressed here is different from the cost function used in connection with the individual firm. In that case, it was possible to express the minimum cost of producing all goods as 
the sum of individual commodity cost functions (Hall 1973). However, each of the components of the total cost function in (3.5a) depended on an arbitrary vector of allocatable fixed inputs assigned to that commodity. The optimal allocation of fixed factors to the production of individual commodities was obtained by maximizing (3.5a) subject to a constraint on the total quantity of each fixed factor available. In this case, the vector $\bar{Z}$ which appears in the sectoral cost function represents the total quantity of each fixed factor, and not its allocatable components. Thus, the cost function for the individual firm is derived as

$$
C^{\star}(Q, R, \bar{Z}, \bar{E})=\operatorname{Minimize} C=\sum_{i=1}^{m} C_{i}^{*}\left(Q, r, Z_{i 1}, \ldots, Z_{i k}, \bar{E}\right)
$$

subject to

$$
\sum_{i=1}^{m} z_{i h}=\bar{z}_{h} \quad \text { for all } h
$$

Corresponding to this, the aggregate cost function for $a=(1, \ldots, b)$ producers could then be written as

$$
C^{*}(Q, r, \bar{Z}, \bar{E}) \approx \sum_{a=1}^{b} C_{a}^{\star}(Q, r, \bar{Z}, \bar{E})
$$

for which Equation (3.10) is an approximation.

In fact, no well-defined function such as Equation (3.10) wil.1 actually exist except under very special conditions. Nevertheless, it is extremely useful to employ this type of function in aggregate models because the first partial derivatives of the cost function with respect to each commodity output provide a functional representation of the marginal cost curves, illustrated in Figure 3.4, for each commodity market.

The multi-market, or sectoral, model described by (3.10) has a single set of first order conditions, namely 


$$
\frac{\partial W}{\partial Q_{i}}=P_{i}(Q, \bar{H})-\frac{\partial C^{\star}}{\partial Q_{j}}=0 \quad \text { for all } i
$$

which simply states that the maximum value of the sum of consumer and producer surplus will be obtained when the marginal cost of producing the last unit of the ith commodity is equal to the price at which it is demanded by consumers.

The first order conditions expressed above can be written in implicit functional form as

$$
P_{i}^{\star}(Q, \bar{H})-C_{i}(Q, r, \bar{Z}, \bar{E})=0 \quad \text { for all } i
$$

where $C_{j}($ ) represents the marginal cost of producing the $i$ th commodity. Solving this system of equations for $Q_{i}$ gives

$$
Q_{i}=Q_{i}^{\star}(\bar{H}, r, \bar{Z}, \bar{E}) \quad \text { for all } i
$$

These functions can then be substituted back into the indirect demand functions to obtain

$$
P_{i}=P_{i}^{\star}(\bar{H}, r, \bar{Z}, \bar{E}) \quad \text { for all } i
$$

Thus, exogenous changes in either the variable input prices, the quantities of fixed factors, the values of exogenous variables contained in the vector, $\bar{H}$, or the state of the environment have the potential to alter the equilibrium prices and quantities that prevail in all agricultural markets; even if these changes originate in only one or a small number of markets. This phenomenon occurs on the demand side because a change in the price of one good will cause consumers to change not only their consumption of that good, but al so the consumption of substitute and complimentary goods. These relationships will be dealt with more fully in the last section of this 
chapter. On the supply side, a change in the marginal cost of producing a single commodity can influence the marginal cost of producing other commodities through adjustments in allocatable fixed factors, such as land, which are used in conjunction with the production of more than a single good. This last feature of agricultural production constitutes a form of jointness in agricultural production (Shumway et al. 1981) which has important implications for modeling the effects of $\mathrm{CO}_{2}$-induced environmental changes in the agricultural sector. In particular, it suggests the need to conduct this analysis using models of the agricultural sector which integrate the behavior of consumers and producers in many different comodity markets, rather than relying on models which are oriented towards a single commodity.

$\mathrm{CO}_{2}$-induced changes in the equilibrium prices and levels of output in agricultural commodity markets will invariably be reflected in changes in the profits which producers earn in these markets. In order to determine the magnitude of this impact one can substitute the aggregate price and quantity relationships from (3.14) into the sectoral profit function

$$
\Pi=\sum_{i=1}^{m} P_{i} Q_{i}-C^{\star}(Q, r, \bar{Z}, \bar{E})
$$

which gives

$$
\Pi=\sum_{i=1}^{m} P_{i}^{\star}(\bar{H}, r, \bar{Z}, \bar{E}) Q_{i}^{\star}(\bar{H}, r, \bar{Z} \bar{E})-C^{\star}[Q(\bar{H}, r, \bar{Z}, \bar{E}), r, \bar{Z}, \bar{E}]
$$

The effect of a small change in $\bar{E}$ upon $\Pi$ can then be expressed by the partial derivative

$$
\frac{\partial \Pi}{\partial E}=\sum_{i=1}^{m}\left[\frac{\partial P_{i}^{*}}{\partial E} Q_{i}^{\star}+\frac{\partial Q_{i}^{*}}{\partial E}\left(P_{i}^{\star}-\frac{\partial C^{*}}{\partial Q_{i}^{*}}\right)\right]-\frac{\partial C^{*}}{\partial E}
$$


By using (3.13a) the expression inside the parentheses can be eliminated, giving as a result the much simpler expression

$$
\frac{\partial \pi}{\partial E}=\sum_{i=1}^{m} \frac{\partial P_{i}^{*}}{\partial E} Q_{i}^{\star}-\frac{\partial C^{*}}{\partial E}
$$

where

$$
\frac{\partial P_{i}^{\star}}{\partial E}=\sum_{i=1}^{m} \frac{\partial P_{i}^{\star}}{\partial Q_{1}^{\star}} \frac{\partial Q_{i}^{*}}{\partial E} \quad \text { for } i, 1=1, \ldots, m
$$

The first set of terms on the right-hand side of (3.15b) represents the effect of a change in $\bar{E}$ on the revenues that are obtained from the sale of agricultural commodities in all markets. In each market, the effect of a $\mathrm{CO}_{2}$-induced environmental change on revenue will depend upon the influence of changes in the state of the environment on the equilibrium price in that market, weighted by the level of commodity output. Other things being equal, then, the magnitude of the environmental impact on revenue in each market will depend on the level of output. However, the effect of changes in the state of the environment on each market price as shown in Equation (3.15c) is not direct. Rather, changes in $P_{i}^{\star}$ in one market will depend on changes on output in that market and other markets which, in turn, are caused by changes in the state of the environment. Thus, the effect of $\mathrm{CO}_{2}$ on output in one market will impact upon the determination of prices and revenues in the other markets to which it is linked indirectly through consumption and directly through joint production. The last term in Equation (3.15b) represents the effect of a change in the environment upon the cost of production in the entire agricultural sector. It is not divisible into separate market components because of the jointness in production due to allocatable fixed factors.

The existence of inter-market effects on revenue makes it impossible to make any refutable statements about the direction of the change in agricultural 
sector revenues that would occur as a result of specifically defined changes in $\bar{E}$. This would be true even if these changes were all positive in the sense that increases in the ambient concentration of $\mathrm{CO}_{2}$ resulted in increased output (and lower prices) in all agricultural markets simultaneously. What is at issue in this specific case, as well as generally, is an empirical problem related to the price elasticity of demand and supply for agricultural commodities. Historically, the demand and supply of many agricultural commodities have been extremely price inelastic. Typically, this has meant that when the supply curve for an agricultural commodity shifts out, as it might be expected to under "favorable" conditions, prices have fallen but output has increased at smaller proportion so that revenues have declined. This is caused by inelastic demands. At the same time, production costs decrease. However, if the supply curve is also inelastic, then costs will not fall as fast revenues and total profits will decline, rather than increase. This type of phenomenon is frequently associated with technological change in the agricultural sector (Hayami and Ruttan 1971), whose effect on the supply curve of an agricultural commodity is not conceptually very much different than the effect of "favorable" $\mathrm{CO}_{2}$-induced environmental changes.

\subsection{INDIRECT IMPACTS OF CO 2 -INDUCED ENVIRONMENTAL CHANGES}

The distinction made between direct and indirect impacts in this report is based in the concept of linkages between firms which engage in agricultural production and the rest of the economy. Impacts that are transmitted from the agricultural sector back through factor and input markets can be identified in terms of backward linkage effects. Impacts that are transmitted from the agricultural sector through the demand for agricultural commodities by food processors and consumers can be identified in terms of forward lirikage effects. In some cases, these indirect effects will be confined to a large extent within the agricultural sector. This is particularly true of backward linkages to input suppliers which provide highly specialized goods and services for the agricultural sector. However, in most cases, the impacts that are transmitted through both kinds of linkages will not only feed back 
into the production of agricultural commodities, they will also carry over into other supply and demand sectors involved in the production or consumption of nonagricultural goods and services.

Because of these feedback effects to agricultural production it is very difficult to classify the indirect impacts of $\mathrm{CO}_{2}$-induced climate changes on the basis of a simple dichotomy between the agricultural sector and other sectors of the economy. Consequently, the operant distinction between direct and indirect economic impacts is that the former are first-order effects, while the latter represent higher-order effects. To show how these impacts work jointly on an intra-sectoral as well as on a inter-sectoral basis would ultimately require the use of a general equilibrium model of the entire economy (Arrow and Debreu 1954; Negishi 1960). Unfortunately, these models are much too general (and, even then, much too complex) to allow anything more than a superficial analysis of the problems that may be encountered if the atmospheric concentration of $\mathrm{CO}_{2}$ should double in the next 50 to 100 years. While the distinction between the impacts of environmental changes which directly affect the production of agricultural comodities and those impacts which are transmitted through forward and backward linkages is consistent with the concept of general equilibrium, separate treatment of direct and indirect effects for analytical purposes represents a necessary concession to partial equilibrium analysis.

\subsubsection{Indirect Impacts on Factor Markets}

Specific indirect impacts that are of potential concern include the effect of changes in the equilibrium price and output levels of agricultural commodities on the supply and price of agricultural labor, land, durable goods including machinery and buildings, and variable inputs such as fertilizer, pesticides, energy, and irrigation water. A detailed analysis of the factors which determine the supply and demand equilibria in all of these markets is we 11 beyond the scope of this report. Consequently, the analytical model used here to describe the indirect effect of $\mathrm{CO}_{2}$-induced environmental changes on factor and input markets will focus on interindustry linkages between agricultural producers and firms which provide both durable and non-durable goods as well as services to the agricultural sector. Furthermore, because 
indirect impacts involve fairly complex economic relationships, the model presented here will be far less general in scope than previous models developed in this chapter.

To illustrate how the impacts of $\mathrm{CO}_{2}$-induced environmental linkages are transmitted to input suppliers through interindustry linkages, consider the case in which a single industry jointly supplies the same two goods to one agricultural market and one nonagricultural market. Trucks and tractors represents one such example. The quantity of trucks and tractors demanded by the agricultural sector is $x_{11}$ and $x_{21}$, respectively, while the corresponding demands from the other sector are $x_{12}$ and $x_{22}$. The market demand functions for trucks and tractors are as follows

\begin{tabular}{|c|c|c|}
\hline Market & Trucks & Tractors \\
\hline Agriculture & $x_{11}=x_{11}^{*}\left(P_{1}, r_{1}, r_{2}, \bar{E}\right)$ & $x_{21}=x_{21}^{*}\left(P_{1}, r_{1}, r_{2}, \bar{E}\right)$ \\
\hline Other & $x_{12}=x_{12}^{*}\left(p_{2}, r_{1}, r_{2}\right)$ & $x_{22}=x_{22}^{*}\left(p_{2}, r_{1}, r_{2}\right)$ \\
\hline
\end{tabular}

where $P_{1}$ and $P_{2}$ denote the market prices of the agricultural and nonagricultural commodities produced using trucks and tractors; $r_{1}$ and $r_{2}$ are the market prices of trucks and tractors, respectively; and $\bar{E}$ is a vector representing the state of the environment which influences agricultural production only. The input demand functions shown above are related to the factor demand equations for individual firms shown earlier in (3.8a). Conceptually, however, the demand equations illustrated here represent aggregate demand functions, showing the quantity of total trucks and tractors which are demanded for specific values of the exogenous variables to which these goods are functionally related.

Trucks and tractors are produced jointly by a large number of firms. The corresponding minimum cost of producing these two inputs can be described by the aggregate cost function 


$$
c=C^{\star}\left(x_{1}, x_{2}, R\right)
$$

where $x_{1}=x_{11}+x_{12}$ and $x_{2}=x_{21}+x_{22}$ represent the total number of trucks and tractors supplied to the two markets and $R$ is a vector of exogenous variables including the price of inputs and the quantities of fixed factors used to produce $x_{1}$ and $x_{2}$.

In order to determine how $\mathrm{CO}_{2}$-induced changes in the environment will affect the production of trucks and tractors, one can form the problem

$$
\begin{aligned}
\text { Maximize } w= & \oint_{0}^{x_{i 1}^{*}}\left\{\sum_{i=1}^{2} r_{i 1}\left(P_{1}, x_{11}, x_{21}, \bar{E}\right) d x_{i 1}\right\}+ \\
& \oint_{0}^{x_{i 1}^{*}}\left\{\sum_{i=1}^{2} r_{i 2}\left(P_{2}, x_{12}, x_{22}\right) d x_{i 2}\right\}- \\
& c^{\star}\left(x_{1}, x_{2} \bar{R}\right)
\end{aligned}
$$

This rather formidable looking problem is directly analogous to the market supply and demand problem for agricultural comodities formulated earlier in (3.10), and illustrated for a single good in Figure 3.4. There are, however, two differences. The first is that the term $W$ now refers to the sum of (a) producer surplus in the two cormodity markets and (b) the surplus associated with the production of trucks and tractors which accrues to firms in that sector. This is illustrated in Figure 3.5 for a single input, trucks, in the agricultural market. The aggregate factor demand curve for trucks is shown by the line DD', while the supply curve for trucks is shown by the line OS. The market supply and demand equilibrium in the use of a single input is $r_{1}^{*}, x_{11}^{\star}$. The area $r_{1}^{\star} D M$ represents the benefit to agricultural producers of being able to buy trucks at $r_{1}$ dollars when they would have been willing to to pay as much as $D$ dollars. The area $r_{1}^{\star} \mathrm{MO}$ represents the profit that truck manufacturers earn by being able to sell trucks at $r_{1}^{\star}$ dollars. The sum of 


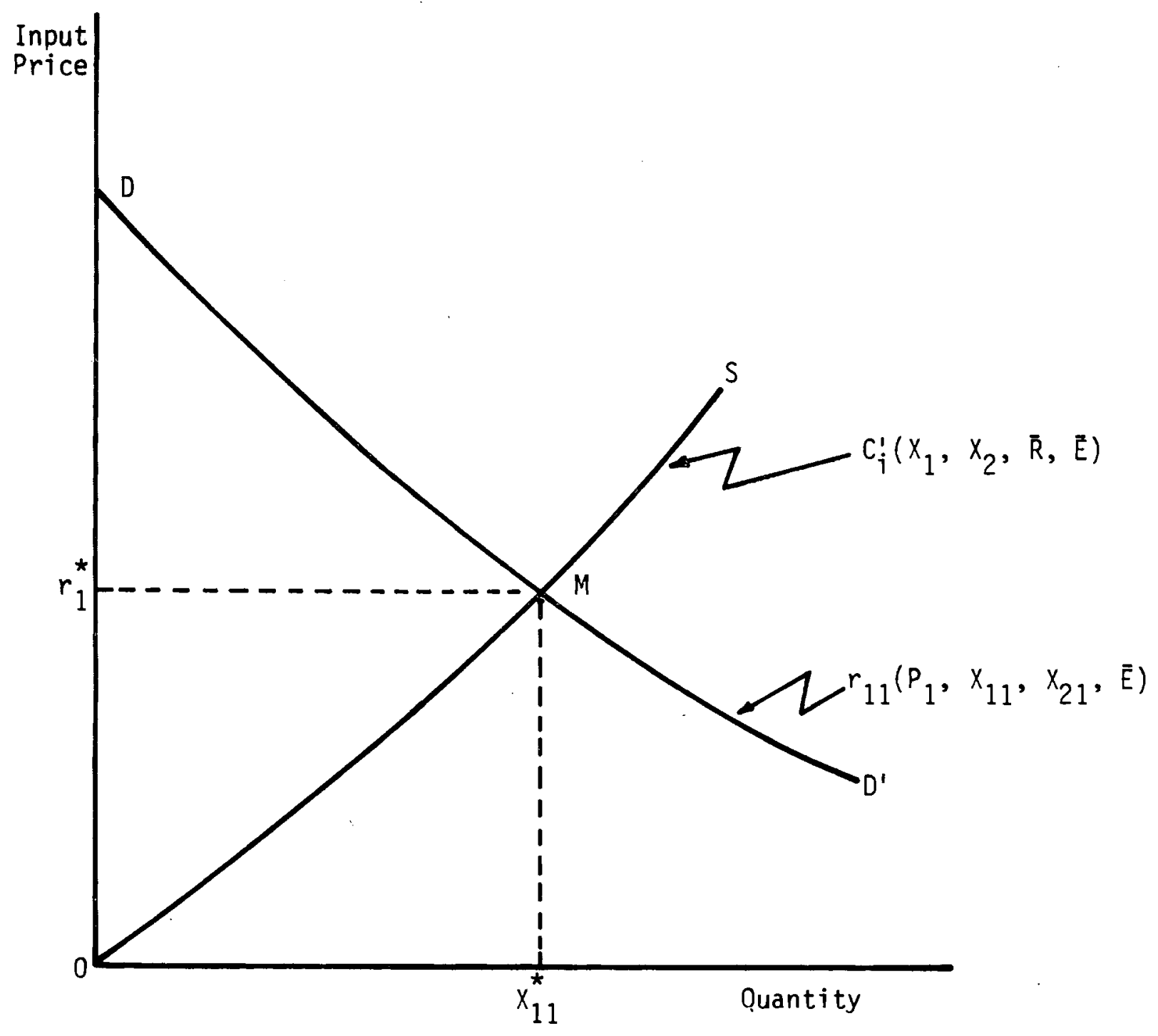

FIGURE 3.5. Determination of Input Price and Quantity Equilibrium for Trucks Used in Agriculture

these two areas in this example would in turn be equal to $W$ for the case of a single input.

The second difference between this problem and (3.10) is in the complicated looking expression for the total willingness to pay of the two producing sectors for trucks and tractors. Each line integral represents the total willingness to pay of producers in the jth sector for trucks and 
tractors. In the case of the single good illustrated in Figure 3.5, the integral

$$
\int_{0}^{x_{11}^{\star}} r_{11}\left(P_{1}, x_{11}, x_{21}, E\right) d x_{11}
$$

represents the total willingness of agricultural firms to pay for trucks, or the area $\mathrm{DMX}_{11}^{*} \mathrm{O}$. However, in the two-good case the total willingness on the part of agricultural firms to pay for trucks (or tractors) is related to the number of tractors (or trucks) demanded, so that the total willingness to pay for both inputs must be expressed in terms of a line integral. The same reasoning applies to the construction of the line integral which represents total willingness to pay for both inputs in the other sector. Note, however, that the demands for these two inputs in one sector are independent of the demands in the other sector; therefore, the total willingness to pay by producers in both sectors for trucks and tractors is simply the sum of the two line integrals. Under more realistic conditions, the consumption of trucks and tractors would be interrelated. However, incorporating this assumption into the problem, while it may add realism, is not necessary to show how $\mathrm{CO}_{2}$-induced changes which affect agricultural production can spill over into other sectors.

The first order conditions for a maximum in (3.17) are

$$
\begin{array}{ll}
\frac{\partial W}{\partial X_{i 1}}=r_{i 1}\left(P_{1}, X_{11}, X_{21}, \bar{E}\right)-\frac{\partial C}{\partial X_{i}}=0 & \text { for a } 11 i \\
\frac{\partial W}{\partial X_{i 2}}=r_{i 2}\left(P_{2}, x_{12}, x_{22}\right)-\frac{\partial C}{\partial X_{i}}=0 & \text { for all } i
\end{array}
$$

Thus the maximum value of $W$ is achieved when the marginal cost of producing trucks (tractors) is equal to the price at which trucks (tractors) are demanded. The equilibrium price of trucks (tractors) will then be the same in both sectors, although the quantitites demanded will in general be different. 
The relationships expressed in (3.18) can also be represented implicitly by

$$
\begin{array}{ll}
r_{i 1}\left(P_{1}, X_{11}, X_{21}, \bar{E}\right)-C_{i}^{\prime}\left(X_{1}, X_{2}, \bar{R}\right)=0 & \text { for all } i \\
r_{i 2}\left(P_{2}, X_{12}, X_{21}\right)-C_{i}^{\prime}\left(X_{1}, X_{2}, \bar{R},\right)=0 & \text { for all } i \\
x_{i}-\sum_{j=1}^{2} X_{i j}=0 & \text { for all } i
\end{array}
$$

where $c_{j}^{\prime}()$ is the marginal cost of producing the ith input. This system of equations can be solved for the optimal values of trucks and tractors used in production by both sectors, which is

$$
x_{i j}=x_{i j}^{*}\left(P_{1}, P_{2}, \bar{R}, \bar{E}\right)
$$

and these expressions can be substituted back into the indirect demand functions to obtain the functional relationship governing input prices, or

$$
r_{i}=r_{i}^{*}\left(P_{1}, P_{2}, \bar{R}, \bar{E}\right)
$$

The important conclusion to be drawn here is that changes in $\bar{E}$ will not only affect the equilibrium prices and quantities demanded of trucks and tractors in the agricultural sector, but in the other sectors as well. This illustrates how $\mathrm{CO}_{2}$-induced environmental changes can be transmitted from the agricultural sector through interindustry linkages to input suppliers whose production may not be affected directly by a rise in $\mathrm{CO}_{2}$. These effects would, in turn, be transmitted even further backward through the interindustry linkage structure of the economy, in this case from truck and tractor manufacturers to the ir parts suppliers and from these firms to the stee 1 , rubber, and textile industries, etc. 
In the previous problem; the prices of agricultural and nonagricultural commodities, $P_{1}$ and $P_{2}$, were regarded as fixed, whereas in the context of the analys is of market behavior in Section 3.3.2 input prices were regarded as fixed. In general, however, the fixed price assumption for either inputs or outputs is not consistent with the notion of general equilibrium in all markets. If this assumption is dropped in both cases then it can be seen from (3.20) that $X$ and $r$ depend on $P$ and from (3.14) that $Q$ and $P$ depend on $r$. Therefore, in general equilibrium, a change in the environment which affects the price and use of an input to production will cause an adjustment to be made in the prices and output levels of the commodities in whose production the input is used. Changes in commodity prices and output levels will in turn cause some adjustment to be made in the prices and output levels of the original set of inputs, as well as in the prices and output levels of other commodities that are. sensitive to these changes. Thus, prices and output levels outside the agricultural sector may be affected and these changes would induce further adjustments to be made in the prices and quantity of inputs used in these markets.

While $\mathrm{CO}_{2}$-induced environmental changes can be expected to produce a kind of "ripple effect" through the economy, like the one described above, this chain of causation should not be viewed as being specific to $\mathrm{CO}_{2}$, nor as an indication that, because other sectors of the economy can be affected by $\mathrm{CO}_{2}$-induced environmental changes, these effects will be uniform in direction or magnitude in all sectors. Again, these kinds of effects can only be accurately quantified using a price-endogenous general equilibrium model of the macroeconomy.

\subsubsection{Impacts on the Demand for Agricultural Commodities}

In Section 3.3.2 an effort was made to show how the effect of $\mathrm{CO}_{2}$-induced environmental changes, which initially affected the supply and demand for a single agricultural commodity, could spill over into other agricultural commodity markets and influence the determination of equilibrium prices and output levels in the latter. In this section, the results obtained there will be extended to illustrate how the same type of phenomenon can occur in connection with the demand for nonagricultural commodities, as well. In 
order to maintain consistency with the analysis in Section 3.3.2, this section will focus on the final demand for agricultural commodities with the understanding that the same effects can be expected to occur in connection with intermediate demands for these products, as we 11.

At the risk of reducing the generality of the mode 1 used here, assume that households consume two aggregate goods, consisting of food and all other goods. The aggregate demand functions for these two comodities can be expressed as

$$
Q_{i}=Q_{i}^{\star}\left(P_{1}, P_{2}, \bar{H}\right) \quad \text { for all } i
$$

where $P_{1}$ and $P_{2}$ are the aggregate prices of food and the remaining composite good and $\bar{H}$ represents a vector of exogenous variables, including per capita income, which influence the demands for these two commodities. The minimum cost of producing the two goods can be represented by the additive (i.e., non-joint) cost function

$$
C=C_{1}^{*}\left(Q_{1}, \bar{R}_{1}, \bar{E}\right)+C_{2}^{*}\left(Q_{2}, \bar{R}_{2}\right)
$$

where $R_{i}$ represents a vector of exogenous variables including variable input prices and the quantities of fixed factors used to produce the ith commodity, and where $\bar{E}$, the state of the environment, directly influences the production of food, only.

Consistent with this information, one can form the problem

$\operatorname{Maximize} W=\oint_{0}^{Q^{\star}}\left\{\sum_{i=1}^{2} P_{i}\left(Q_{1}, Q_{2}, \bar{H}\right) d Q_{i}\right\}-C_{1}^{*}\left(Q_{1}, \bar{R}_{1}, \bar{E}\right)-C_{2}^{\star}\left(Q_{2}, R_{2}\right)$

where $W$ represents the sum of consumer and producer surplus, previously defined, and $P_{i}\left(Q_{1}, Q_{2}, \bar{H}\right)$ is the indirect demand function for the ith commodity, relating the price of each aggregate good to the quantities consumed of both goods and the vector $\bar{H}$. 
The first order conditions for (3.22) are

$$
\frac{\partial W}{\partial Q_{i}}=P_{i}\left(Q_{1}, Q_{2}, \bar{H}\right)-\frac{\partial C_{i}}{\partial Q_{i}}=0 \quad \text { for all } i
$$

which can be expressed in implicit form by

$$
\begin{aligned}
& P_{1}\left(Q_{1}, Q_{2}, \bar{H}\right)-C_{1}\left(Q_{1}, \bar{R}_{1}, \bar{E}\right)=0 \\
& P_{2}\left(Q_{1}, Q_{2}, \bar{H}\right)-C_{2}\left(Q_{2}, \bar{R}_{2}\right)=0
\end{aligned}
$$

From these two relationships one can then derive the optimal values for $Q_{1}$ and $Q_{2}$ versus functions of the exogenous variables, $\bar{H}, \bar{R}$ and $\bar{E}$, or

$$
\begin{aligned}
& Q_{1}=Q_{1}^{*}\left(\bar{H}, \bar{R}_{1}, \bar{R}_{2}, \bar{E}\right) \\
& Q_{2}=Q_{2}^{*}\left(\bar{H}, \bar{R}_{1}, \bar{R}_{2}, \bar{E}\right)
\end{aligned}
$$

Subsequently, these functions can be substituted for $Q_{1}$ and $Q_{2}$ in the indirect demand functions in order to obtain the optimal values of $P_{1}$ and $P_{2}$, which are

$$
\begin{aligned}
& P_{1}=P_{1}^{*}\left(\bar{H}, \bar{R}_{1}, \bar{R}_{2}, \bar{E}\right) \\
& P_{2}=P_{2}^{*}\left(\bar{H}, \bar{R}_{1}, \bar{R}_{2}, \bar{E}\right)
\end{aligned}
$$

Thus, a $\mathrm{CO}_{2}$-induced environmental change that initially impacts the production of agricultural commodities has the potential to influence the equilibrium prices and output levels that prevail in nonagricultural markets, as well. From the standpoint of consumer demands, the magnitude of this 
impact on the consumption of nonagricultural commodities will depend on the structure of consumer preferences embodied in the direct and indirect demand functions. In this example, the demand functions for both goods expressed in (3.21a) purposely contained arguments in both $P_{1}$ and $P_{2}$. This means that an exogenous change in the price of either good will affect the consumption of both goods. It also implies that the effect of a $\mathrm{CO}_{2}$-induced environmental change on the final (and intermediate) demand for agricultural and nonagricultural commodities will depend to a large extent on how sensitive consumption is to changes in the prices of both agricultural and nonagricultural commodities.

In order to demonstrate this, one can substitute (3.25) for $P_{1}$ amd $P_{2}$ in the demand equations expressed by (3.21a). The result is

$$
Q_{i}=Q_{i}\left[P_{1}^{\star}(\bar{H}, \bar{R}, \bar{E}), P_{2}^{\star}(\bar{H}, \bar{R}, \bar{E}), \bar{H}\right]
$$

The effect of small change in $\bar{E}$ on $Q_{j}$ can then be expressed as

$$
\frac{\partial Q_{i}}{\partial E}=\frac{\partial Q_{i}}{\partial P_{1}^{\star}} \frac{\partial P_{1}^{\star}}{\partial E}+\frac{\partial Q_{j}}{\partial P_{2}^{\star}} \frac{\partial P_{2}^{\star}}{\partial E}
$$

Thus, the effect of a $\mathrm{CO}_{2}$-induced change in the state of the environment on the consumption of the ith commodity is the weighted sum of the effects of that change on $P_{1}$ and $P_{2}$, where the weights correspond to the partial derivatives $\partial Q_{1} / \partial P_{1}$ and $\partial Q_{1} / \partial P_{2}$, which measure the sensitivity of consumption to smal1 changes in the own- and cross-prices.

Equation (3.27a) can also be expressed in terms of the effect of a $1 \%$ change in $E$ on the percentage change in $Q_{j}$ as follows

$$
e_{i}=\frac{\partial Q_{i}}{\partial E} \frac{E}{Q_{i}}=\frac{\partial Q_{1}}{\partial P_{1}^{\star}} \frac{P_{1}^{*}}{Q_{i}} e_{p 1}+\frac{\partial Q_{i}}{\partial P_{2}^{\star}} \frac{P_{2}^{*}}{Q_{i}} e_{p 2}
$$


which can be generalized to the case of $N(i, j=1, \ldots, N)$ agricultural and nonagricultural goods as

$$
e_{i}=\sum_{j=1}^{N} \frac{\partial Q_{i}}{\partial P_{j}^{*}} \frac{P_{j}^{*}}{Q_{i}} e_{p j}
$$

where

$$
e_{p j}=\frac{\partial P}{\partial E} \frac{E}{P_{j}}
$$

Thus, $e_{p j}$ represents the effect of a $1 \%$ change in $\bar{E}$ on the percentage change in $P_{j}$. In general, the effect of changes in $\bar{E}$ on $P_{j}$ cannot be determined $a$ priori. However, the weights in (3.28b) now represent the own and cross-price elasticities of demand for $Q_{i}$ with respect to $P_{j}$. Empirical estimates of these elasticities for individual agricultural commodities, as well as for categories of aggregate agricultural and nonagricultural comodities are widely available from a variety of sources.

At least at the level of consumer demands, then, this information can be used on a preliminary basis to make some tentative assessments about the relative magnitudes of the impact of $\mathrm{CO}_{2}$-induced changes in the state of the environment on the final demands for agricultural and nonagricultural commodities. For example, if the cross-price elasticity of the consumer demand for automobiles with respect to the price of food is very small, then a $\mathrm{CO}_{2}$-induced indirect environmental change which does not directly affect the production of automobiles, but which does influence the price of food, will have a relatively small effect, other things being equal, on the consumption of automobiles.

The example used here has focused on final demands, but as was suggested earlier, can also be applied to the analysis of the intermediate demand for agricultural comodities with only minor revisions. The elasticity relationship expressed in (3.28b) is general enough to apply to both types of demand. The integration of both intermediate and final demands into a systematic framework is much more difficult to accomplish. It requires a 
model that is able to include all of the adjustments that can occur simultaneously in many markets in response to a $\mathrm{CO}_{2}$-induced environmental change, but which is also specific enough to focus on commodity markets within the agricultural sector as the source of these changes. The problems of integrating numerous markets that are connected by interindustry 1 inkages into a more general framework than is provided here will be discussed in more detail in Chapter 7.0 of this report.

\subsection{SUMMARY OF DIRECT AND INDIRECT ECONOMIC IMPACTS}

The analysis of the economic impact of $\mathrm{CO}_{2}$-induced environmental changes must begin at the level of the individual producer because it is at this point that the natural and physical environment is connected to the economic environment. This linkage can be modeled more explicitly in terms of a production function

$$
Q=f(X, \bar{Z}, \bar{E})
$$

which shows the maximum output of the commodity $Q$ that can be obtained by combining variable inputs $(X)$, such as fertilizer, water and labor, and fixed inputs $(\bar{Z})$, such as land and stocks of durable goods, given the state of the environment as $\bar{E}$. Thus, the environment is treated as an input to production, and it is therefore through $\bar{E}$ that changes in the ambient concentration of $\mathrm{CO}_{2}$ will enter the production process.

Equation (3.29a) does not constitute a model of production, it merely defines the technology of the firm. In order to find out how $\mathrm{CO}_{2}$-induced changes in $\bar{E}$ may affect production it is necessary to incorporate the technology of the firm into a planning framework that is consistent with rational behavior on the part of producers. For example, if producers are profit maximizers, an economic model consistent with this objective can be expressed as

$$
\text { Maximize II }=\operatorname{Pf}(X, \bar{Z}, \bar{E})-r X
$$


where $\pi$ is profit, $P$ is the price of the commodity, and $r$ is the market price of the variable input, $x$. The solution to this planning problem is represented by an input demand function

$$
X=X^{*}(P, r, \bar{Z}, \bar{E})
$$

which relates the optimal use of this input to the exogenous variables in Equation (3.30), exogenous meaning that the producer has no direct control over their values during the planning period. Thus, the first conclusion that can be arrived at is that changes in the state of the environment may affect input use. This will include not only variable input usage, but also the use of land which is variable in the sense that a given quantity can be allocated to different crops.

The second conclusion that can be drawn is that changes in the state of the environment will also affect production by the firm. This can be seen by substituting the input demand function from Equation (3.30) back into the production function for $x$. The result

$$
Q=f\left[X^{*}(P, r, \bar{Z}, \bar{E}), \bar{Z}, \bar{E}\right]
$$

is the supply function for $Q$, which relates the optimal value of output to the exogenous variables, $P, r, \bar{Z}$ and $\bar{E}$. The effect of a small change in $\bar{E}$ upon $Q$ can be expressed by the partial derivative of $Q$ with respect to $\bar{E}$, or

$$
\frac{\partial Q}{\partial E}=\frac{\partial f}{\partial X^{\star}} \frac{\partial X^{\star}}{\partial E}+\frac{\partial f}{\partial E}
$$

This shows that the impact of changes in the state of the environment caused by $\mathrm{CO}_{2}$ can be decomposed into: (1) a term which represents the indirect effect of a change in $E$ on output due to the influence of $E$ on input usage, and (2) a second term that captures the direct effect of a change in $\bar{E}$ on output through the parameters of the original production function $f()$. 
When one moves from the firm to the market, it is no longer possible to treat comodity prices as fixed. In competitive markets, no single producer can substantially affect comodity prices; however, changes in output by a large number of producers will influence the commodity prices that prevail in these markets. The direct effect of $\mathrm{CO}_{2}$-induced environmental changes on market prices, output and producer profits requires more detailed mathematical treatment than can be given in this sumary. However, the conclusions that emerge from Section 3.3.2 can be stated in fairly simple terms. The first conclusion is that, even if the demands for agricultural commodities are not interdependent, a $\mathrm{CO}_{2}$-induced change in the environment which directly affects the production of only one cormodity will have an impact on the market prices and output levels of other commodities. This effect is transmitted between markets due to the jointness in production that is created when a fixed amount of land can be allocated to a number of competing crops or to any number of other causes of joint production. If demands are interdependent (for example the purchase of livestock depends on the price of feedgrains), then the effect of $\mathrm{CO}_{2}$ on the production of one will commodity will be transmitted to other commodities by these interdependencies as we 11 .

The second conclusion is directly related to the first: because of these intermarket effects it is not possible to determine on an a priori basis what the net effect of even very isolated changes in the state of the environment will be on market prices, output levels and total profits within the agricultural sector. In addition, it was also suggested that, even if $\mathrm{CO}_{2}$-induced environmental changes did cause increased production in all agricultural markets, the effect of this may be to reduce, rather than increase profits. This would be a likely occurrence in the case of commodities whose demand and supply curves are price inelastic in the relevant region of consumption and output. In this case, the "favorable" influence of $\mathrm{CO}_{2}$ on production would be likely to produce a decline in both revenue and cost; however, costs would decline by a smaller proportion than revenue and the net effect would be a reduction in profits.

Section 3.4.1 examined the effect of changes in the ambient concentration of $\mathrm{CO}_{2}$ on input and factor markets which are tied to the agricultural sector 
through backward linkages. In this section a simple example was used to show how changes in the ambient concentration of $\mathrm{CO}_{2}$ will affect the aggregate demand for these inputs, resulting in changes in their market prices and levels of outputs. For example, a $\mathrm{CO}_{2}$-induced change in the price of tractors will also affect the price of trucks and other types of equipment that are produced jointly for agricultural and nonagricultural use. Changes in the prices of these goods will, in turn, ripple back through the economy because of backward linkages to other industries. Thus, in a hypothetical case, the price of diesel fuel injection systems will be affected by $\mathrm{CO}_{2}$-induced environmental changes in the price of tractors, so that ultimately the cost of diesel automobiles would also be affected. In an economy characterized by a complex interindustry linkage structure, these second-order economic impacts are likely to be very wide-spread. The actual magnitude of the price and output changes that will occur in industries linked directly or indirectly to the agricultural sector is an empirical question, not a theoretical one.

Section 3.4.1 examined the indirect effect of $\mathrm{CO}_{2}$-induced environmental changes on consumer demand for nonagricultural commodities. Impacts on consumers will take the form of price changes in either agricultural and/or nonagricultural commodities (due to interindustry linkage effects). Because the budget of the individual consumer is assumed to be fixed, nonproportional price changes (holding income constant) will cause individual consumers to redistribute their incomes consistent with utility maximization. At the aggregate level, these effects will occur simultaneously such that, if the aggregate demand for food is related to the price of nonfood commodities, then the equibrium prices and consumption levels of all these interrelated goods will depend upon the state of the environment.

The ultimate conclusion to be drawn from the theoretical analys is of the direct and indirect economic impacts of $\mathrm{CO}_{2}$-induced environmental changes is that instead of being able to narrow down the range of impacts, it is difficult to identify markets which will not be affected by these changes. It is clear, however, that the magnitude of these impacts will not be uniform (even if the effect of $\mathrm{CO}_{2}$ on the environment is). Moreover, the intensity 
of the impacts related to $\mathrm{CO}_{2}$-induced changes in the environment will depend upon how responsive prices are in one market or sector to direct and indirect $\mathrm{CO}_{2}$-induced output and consumption changes in the sectors to which they are linked. These relationships cannot be quantified on the basis of theory.

That is why one must ultimately turn to economic models, not only to describe these relationships, but also to show how these relationships will determine the overall impacts of $\mathrm{CO}_{2}$-induced environmental changes on the agricultural sector and the rest of the economy. 


\section{REFERENCES}

Aoki, M. and K. Yabuki. 1977. "Studies on the Carbon Dioxide Enrichment for Plant Growth, VII. Changes in Dry Matter Production and Photosynthet ic Rate of Cucumber During Carbon Dioxide Enrichment." Agricultural Meteorology $18(3): 475-485$.

Arrow, K. J. and G. Debreu. 1954. "Existence of an Equilibrium for a Competitive Economy." Econometrica 22(2)265-290.

Baker, D. N. and J. D. Hesketh. 1967. "Light and Carbon Assimilation by Plant Communities." Crop Science, 3:(2)285-293.

Baker, D. N. et al. 1981. The Impact of Increased $\mathrm{CO}_{2}$ on Photosynthesis and Agricultural Productivity, A Research Proposal. SEA-AR, U.S. Department of Agriculture, Sourthern Region and Department of Agricultural Engineering, Clemson University.

Bauer, A. 1972. Effect of Water Supply and Seasonal Distribution of Spring Wheat Yields. North Dakota State University, Agricultural Experiment Station Bu17et in 490, Bismarck, North Dakota.

Black, C. C. and T. M. Chen. 1969. "Biochemical Basis for Plant Competition." Weed Science $17(3): 338-344$.

Bollman, F. and G. Hellyer. 1974. The Economic Consequences of Projected Temperature Changes in Climatically Sensitive Wheat Growing Areas of the Canadian Prairie. Development and Resources Corporation, Sacramento, California.

Boyer, J. S. and H. C. McPherson. 1975. "Physiology of Water Deficits in Cereal Crops." Advanced Agronomy 27(1):1-23.

Broecker, W. et al. 1980. "Modeling the Climate System." In Proceedings of the Carbon Dioxide and Climate Research Program. U.S. Department of Energy, pp. 43-100. CONF-8004110-UC-11, National Technical Information Service, Springfield, Virginia.

Chen, K. et al. 1980. "Carbon Dioxide from Fossil Fuels." Energy Policy December 1980, pp. 318-330.

Council on Environmental Quality. 1981. Global Energy Futures and the Carbon Dioxide Problem. U.S. Government Printing Office, Washington, D.C.

Dupuit, J. 1844. "De la Mesure de l'Utilitie des Travaux Publics." Annales des Ponts et Chaussees 8:163-221.

Evans, L. T. (ed.) 1975. Crop Physiology. Cambridge University Press, Cambridge, England. 
Evans, L. T. and I, F, Wardlow. 1976. "Aspects of the Comparative Physiology' of Grain Yield in Cerea ls." Advanced Agronomy 28:301-359.

Fick, G. W. et al. 1975. "Sugar Beets." In Crop Physiology, ed. L. T. Evans, pp. 259-295, Cambridge University Press, Cambridge, England.

Fischer, R. A. 1975. "Yield Potential in Spring wheat and the Effects of Shading." Crop Science 15(4):609-613.

Fisher, R. A. and R. M. Hagen. 1965. "Plant Water Relations, Irrigation Management and Crop Yield." Experimental Agriculture 1(1):161-177.

Flohn, H. 1980. Possible Climatic Consequences of a Man-Made Global Warning. International Institute for Applied Systems Analysis, Luxenburg, Austria.

Gaastra, P. 1959. "Photosynthesis of Crop Plants as Influenced by Light, Carbon Dioxide, Temperature, and Stomatal Diffusion Resistance." Meded. Landb. Hoogesh., Wagen ingen 59(1):1-68.

Gage, S. H. and M. R. Mukerji. 1978. "Crop Losses Associated with Grasshoppers in Relation to Economics of Crop Production." Journal of Economic Entomology $71(3): 487-498$.

Gifford, R M. 1979. "Growth and Yield of $\mathrm{CO}_{2}$-Enriched Wheat Under Water Limited Conditions." Australian Journal of Plant Physiology $6(3): 367-378$.

Goudrian, J. and H. H. van Laar. 1978. "Relations Between Leaf Resistance, $\mathrm{CO}_{2}$ Concentration and $\mathrm{CO}_{2}$ Assimilation in Maize, Beans, Lalang Grass, and Sunflower." Photosynthetica 12(2):241-249.

Hall, R. F. 1973. "The Specification of Technology with Several Kinds of Output." Journal of Political Economy $81(4): 878-892$.

Hansen, J. et a 1. 1981. "Climate Impact of Increasing Atmospheric Carbon Dioxide." Science 213, pp. 957-966.

Hayami, Y. and V.W. Ruttan. 1971. Agricultural Development: An International Perspective. The Johns Hopkins University Press, Baltimore, Maryland.

Hotelling, H. 1932. "Edgeworth's Taxation Paradox and the Nature of Demand and Supply Functions." Journal of Political Economy 40(5):577-616.

Hotelling, H. 1938. "The General Welfare in Relation to Problems of Taxation and of Railway and Utility Rates." Econometirca 6(2):242-269.

Idso, S. 1980. "The Climatological Significance of a Doubling of Earth's Atmospheric Carbon Dioxide Concentration." Science 207, pp. 1462-1463. 
Keeling, C. D. et al. 1976. "Atmospheric Carbon Dioxide Variations at Manna Loa Observatory, Hawaii and the South Pole." Tellus 28, pp. 538-564.

Kellogg, W. W. 1978. "Global Influences of Mankind on Climate." In Climatic Change, ed. J. Gribbin, pp. 205-277. Cambridge University Press, Cambridge, England.

Kellog, W. W. 1979. "Influences of Man on Climate." Annual Review of Earth and Planetary Sciences $7(1): 63-92$.

Kramer, P. J. 1981. "Carbon Dioxide Concentration, Photosynthesis, and Dry Matter Production." Bio Science 31(1):29-33.

Lamb, H. H. 1977. Climate: Present, Past and Future. 2 Vols. Methuen, London, England.

Lau, L. J. 1978. "Applications of Profit Functions." In Production Economics: A Dual Approach to Theory and Applications, I, ed. M. Fuss and D. McFadden, pp. 133-216. North Holland, New Amsterdam, The Nether 1 ands.

Ludlow, M. M. and G. L. Wilson. 1971. "Photosynthesis of Tropical Pasture Plants, II, Temperature and Illuminance History." Australian Journal of Biological Sciences 24(5):1065-1075.

Manabe, S. and R. T. Wetherald. 1975. "The Effects of Doubling the $\mathrm{CO}_{2}$ Concentration on the Climate of a General Circulation Model." Journal of Atmospheric Science 32(1):3-15.

Manabe, S. and R. T. Wetherald. 1980. "On the Distribution of Climate Change Resulting from an Increase in $\mathrm{CO}_{2}$ Content of the Atmosphere." Journal of Atmospheric Sciences $37(1): 99-118$.

Marsha11, A. 1890. Principles of Economics. Macmillian, London, England.

Mauney, J. R. et al. 1978. "Relationship of Photosynthetic Rate to Growth and Fruiting of Cotton, Soybean, Sorghum, and Sunflower." Crop Science $18(2): 259-263$.

McFadden, D. 1978. "Cost, Revenue, and Profit Functions." In Production Economics: A Dual Approach to Theory and Applications, I. eds.M. Fuss and D. McFadden, pp. 3-110. North Holland, Amsterdam, The Netherlands.

National Research Council. 1979. Carbon Dioxide and Climate: A Scientific Assessment. National Academy of Sciences, Washington, D.C.

Negishi, T. 1960. "Welfare Economics and Existence of an Equilibrium for a Compet itive Economy." Metroeconometrica 22(1):92-97.

Newe 11, R. E. and T. G. Dopplick. 1979. "Questions Concerning the Possible Influence of Anthropogenic $\mathrm{CO}_{2}$ on Atmospheric Temperature." Journal of Applied Meteorology 18(4):822-825. 
Pallas, J. E. 1965. "Transpiration and Stomatal Opening with Changes in Carbon Dioxide Content of the Air." Science 147, pp. 171-173.

Patterson, D. T. and E. P. Flint. 1980. "Potential Effects of Global Atmospheric $\mathrm{CO}_{2}$ Enrichment on the Growth and Competitiveness of $\mathrm{C}_{3}$ and $\mathrm{C}_{4}$ Weed and Crop Plants." Weed Science 28(1):71-75.

Pimenta1, D. 1976. "World Food Crisis: Energy and Pests." Bulletin of the American Entomological Society 22(1):20-26.

Pimenta1, D. 1980. "Increased $\mathrm{CO}_{2}$ Effects on the Environmental and in Turn on Agricultural and Forestry." In Workshop on Environmental and Societal Consequences of a Possible $\mathrm{CO}_{2}$-Induced Climate Change. U.S. Department of Energy, pp. 260-274. CONF-7904143, National Technical Information Service, Springfield, Virginia.

Pimental, D. and M. Pimental. 1978. "Dimensions of the World Food Problem and Losses to Pests." In World Food, Pest Losses, and the Environment, ed. D. Pimental, pp. 1-16. Westview Press, BouTder, Colorado.

Ramanathan, V. and J. A. Coakley. 1978. "Cl imate Modeling Through RadativeConvective Models." Review of Geophysics and Space Physics 16(3):465-489.

Ramanathan, V. et al. 1979. "Increased Atmospheric $\mathrm{CO}_{2}$ : Zonal and Seasonal Estimates of the Effect on the Radiation Energy Balance and Surface Temperature." Journal of Geophysical Research 84(4c):4947-4958.

Sagan, C. et al. 1979.: "Anthropogenic Albedo Changes and the Earth's Climate." Science 206, pp. 1363-1367.

Salter, P. J. and J. E. Goode. 1967. Crop Responses to Water at Different Stages of Growth. Commonwealth Agricultural Bureaux, Farham Royal, England.

de Saussure, T. 1804. Recherches Chemiques sur la Vegitation. Paris, France.

Shephard, R. W. 1953. Cost and Production Functions. Princeton University Press, Princeton, New Jersey.

Shephard, R. W. 1970. Theory of Cost and Production Functions. Princeton University Press, Princeton, New Jersey.

Shumway, C. R. et al. "Allocatable Fixed Factors and Jointness in Agricultural Production: Implications for Economic Modeling." Staff Paper DIR81-1, SP-5, Department of Agricultural Economics, Texas A\&M University, College Station, Texas.

Siegenthaler, U., and H. Oeschger. 1978. "Predicting Future Atmospheric Carbon Dioxide Levels." Science 199, pp. 388-375. 
Strain, B. R., ed. 1977. Report of the Workshop on Anticipated Plant Response to Global Carbon Dioxide Enrichment. Duke University, Durham, North Carolina.

Stuiver, M. 1978. "Atmospheric Carbon Dioxide and Carbon Reservior Changes." Science 19, pp. 253-258.

U.S. Department of Energy. 1980. Proceedings of the Carbon Dioxide and Cl imate Research Program Conference. CONF-8004110-UC-11, National Technical Information Service, Springfield, Virginia.

Watts, R. G. 1980. "Climate Models and $\mathrm{CO}_{2}$-Induced Climatic Changes." Climatic Change 2(3):387-408.

Wetherald, R. T. and S. Manabe. 1981. "Influences of Seasonal Variation Upon the Sensitivity of a Model Climate." Journal of Geophysical Research $86(C 2): 1194-1204$.

Wigley, T. M. L. et al. 1980. "Scenario for a Warm, High-CO2 World." Nature 283, pp. 17-22.

de Wit, C. T. et al. 1970. "The Simulation of Photosynthetic Systems." In Prediction and Measurement of Photosynthetic Productivity. Proceedings of the IBP/PP Technical Meeting, ed. I. Setlick, pp. 47-50. Pudoc, Wagen ingen, The Netherlands.

de Wit, C. T. et al. 1978. Simulation of Assimilation, Respiration and Transpiration of Crops. Halsted Press, John Wiley \& Sons, New York, New York.

Wittwer, S. H. 1980. "Carbon Dioxide and Climate Change: An Agricultural Perspective." Journal of Soil and Water Conservation 35(3):116-120.

Wittwer, S. H. 1981. "The Intricate Measure: An Agricultural Perspective on Research Tools for Early Detection of the Biological, Biochemical and Environmental Sensitivities to $\mathrm{CO}_{2}$." Paper presented at the Workshop on First Detection of Carbon Dioxide Effects, June 8-10, 1981, Harper's Ferry, West Virginia.

World Meteorological Organization. 1979. Report of the Meeting of CAS Working Group on Atmospheric Carbon Dioxide, November 1979. WMO Project on Research and Monitoring of $\mathrm{CO}_{2}$, Report No. 2, World Meteorological Organization, Geneva, Switzer1and. 


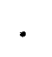




\subsection{VALUATION OF INFORMATION RELATED TO UNCERTAIN ENVIRONMENTAL CHANGES}

The best available scientific evidence suggests that atmospheric concentrations of $\mathrm{CO}_{2}$ are currently increasing (CEQ 1981; Hansen et al. 1981). It has been hypothesized that as a result the mean global temperature could increase two or more degrees Centigrade over the next 50 to 100 years. Should this global warming occur, mean temperatures will not rise uniformly over the planet. In general, increases in average temperatures will be greater the farther the distance from the equatorial latitudes (CEQ 1981). The resulting impact of these temperature changes on the existing geographic distribution of $\mathrm{climate}$ is expected to be even less un if orm (National Research Council 1979). Another possibility is that global warming would be accompanied by a significant increase in the frequency of extreme weather conditions. Other climate changes, such as those relating to precipitation and evaporation, have also been postulated (CEQ 1981). Finally, it has been suggested that these changes in climate will result in major impacts on agriculture over large portions of the globe (Kellog and Schware 1981). The impacts would be beneficial to some areas, while other areas would be adversely impacted.

Should all of these things occur, then adjustments to these events within the agricultural sector can be expected to take many forms. One major response would be for producers to shift to crops that are better suited to the altered climate. Another response would be for plant breeders and seed companies to develop new strains of plants that could better withstand the altered climate, particularly if the frequency and/or severity of extreme weather conditions should increase. Additionally, the climate changes could also be expected to affect agricultural pests, such as insects and weeds. Should the nature of the pest problem change significantly, new plant hybrids and pesticides would need to be developed to counteract the results of this change. Finally, providing new irrigation water supplies to areas that might become "stranded" due to reduced precipitation or to greater seasonal variability in precipitation is still another form of response.

Neither the predicted $\mathrm{climate}$ changes nor the ir impacts on agriculture should be considered as certain to occur. While it is more likely than not 
that average global temperatures will rise due to $\mathrm{CO}_{2}$ buildup in the atmosphere, the timing of these changes, their impact on regional climates, and the resulting effects of regional climatic changes on agricultural production are all highly uncertain. In that context, the major function of $\mathrm{CO}_{2}$ research is to try to reduce the uncertainty that surrounds current hypotheses regarding the impact of $\mathrm{CO}_{2}$ on the biosphere and the resulting impact of $\mathrm{CO}_{2}$-induced environmental changes on the U.S. economy. However, providing more precise information about the myriad impacts of $\mathrm{CO}_{2}$ buildup will not be costless. Therefore, the question naturally arises as to how much of this nation's resources ought to be committed to improving our understanding of the future impacts that will occur due to the continuing addition of carbon to the atmosphere.

The amount of money that is spent on research and the areas in which it is spent in order to provide information about the effects of $\mathrm{CO}_{2}$ buildup will ultimately depend on the objectives that are adopted, explicitly or implicitly, to guide further research. If one of these objectives is economic efficiency, then it is possible to adopt a framework in which decisions about how much to spend, and where, are based at least in part upon the value of this information in helping economic agents within the agricultural sector to adjust to these changes, before they occur, thereby reducing the cost of resources that might have to be committed to avoid unanticipated, catastrophic impacts. However, in order to implement any research strategy based on the objective of economic efficiency, it must be shown that if more certain information is provided in the future it will actually be of some value to economic agents in the agricultural sector, whether or not they are actually willing to pay for this information. Once this has been demonstrated, research investments and priorities can be updated continually based on the potential future value of specific kinds of research.

The analytical methods associated with determining the optimal amount of information that should be produced consistent with the objective of economic efficiency in both static and dynamic situations are well developed (Rausser and Hochman 1979; Zellner and Chetty 1965; Zellner and Geisel 1968; Taylor 1976). However, most of the literature devoted to the application of these 
methods in cases involving anthropogenically based climate changes have focused on the problem of providing the optimal amount of information required for public policy actions to control the man-made causes of these changes (CIAP 1975). While controlling fossil fuel emissions of carbon into the atmosphere is a legitimate object of public policy, not only are the costs associated with regulating fossil burning astronomically high, but there is also considerable doubt about the effectiveness of such measures (CEQ 1981; Chen et al. 1980). It is likely, then, that the current benefits of $\mathrm{CO}_{2}$ research will not lie in the area of providing information for regulatory purposes, but rather will be due to the value of this information insofar as it allows economic agents to adjust more smoothly to altered climatic states than would otherwise be possible.

In that general framework, this chapter has three basic objectives. The first of these is to demonstrate how firms behave under conditions of environmental uncertainty and to present some of the more important factors that will influence their behavior under these conditions. The second objective is to show how information that provides greater certainty about the state of the environment will alter the behavior of firms in such a way that it is possible to derive the demand for information, and as a result place an economic value on it. The third and final objective is to show in a very general and nonmathematical framework how it is possible to develop a research strategy that is based on the value of information.

\subsection{RESPONSES OF AGRIGULTURAL FIRMS TO ENVIRONMENTAL UNCERTAINTY}

Agricultural firms are presumed to be naturally motivated to make wealth-enhancing decisions based on $\mathrm{CO}_{2}$-related information that becomes available to them. These decisions may be made well before any significant climate changes actually occur if, through these decisions, firms can better position themselves to exploit the climate changes. In this section the factors affecting their responses and the variety of responses are discussed. However, before doing this, the exact nature of the uncertainties that are to be examined need to be classified. Currently, the main ingredients of uncertainty associated with the buildup of $\mathrm{CO}_{2}$ in the atmosphere are: 
- the climate changes are expected to become significant in 50 to 100 years, although some scientists believe this will happen sooner (for the latter view see, for example, Hansen et al. 1981);

- the rate at which the ambient concentration of $\mathrm{CO}_{2}$ will increase in the future is known only with considerable uncertainty; and

- given the predicted buildup in atmospheric concentrations of $\mathrm{CO}_{2}$, the impacts on global, regional and local climate are known only with considerable uncertainty.

\subsubsection{Assumptions}

The analysis of this section is based upon the following assumptions:

1. Agricultural firms are wealth-maximizers.

2. All uncertainty can be incorporated within a probability-risk framework; the probabilities may be objective or subjective.

3. Climate is characterized by a joint probability distribution of meteorological events over time (Howe 1980):

$$
h(w(t, s), B),
$$

where $W(t, s)$ is a random vector of meteorological variables that influence crop yields during time $t$ at location $s$, and $B$ is a vector of true parameters associated with this process; namely, the means, variances, covariances and autocorrelation structures.

The first assumption has already been discussed in Section 3.3.1 under the heading of profit maximization. In point of fact, however, agricultural producers are generally risk averse, and as such are more likely to maximize the ir expected utility which is a function of expected wealth and the temporal variance in wealth. The effect of risk aversion on the willingness of firms to pay for information that reduces current uncertainty about the future state of the enviranment (without necessarily affected the expected state) will be discussed more thoroughly in Section 4.2.2. For the moment, however, the 
assumption of wealth maximization will be maintained. The second assumption implies that all uncertainty can be quantified either as objective or subjective risk, as discussed below. Assumption three is that the joint distribution specified in (4.1) characterizes the entire meteorological process. Random variables in the vector $W$ include temperature, precipitation, solar radiation, relative humidity, wind speed and direction, etc. Changes in weather at any location can be described by the daily fluctuations in the actual values of the meteorological variables. In terms of Equation (4.1), climate variability refers to the variance-covariance parameters within a fixed $B$, while climate change refers to changes in $B$ that occur over time due to exogenous influences, such as increases in the ambient concentration of $\mathrm{CO}_{2}$

Implicit in much of the literature on climatic impacts of $\mathrm{CO}_{2}$ is a concept of climate change that focuses almost exclusively on the changes that may occur in the mean values of specific meteorological variables, such as monthly or annual average temperature. This view generally ignores changes that may occur in the other parameters of both the marginal distributions of individual meteorological variables as well as in the joint distribution of the variables represented in (4.1). To the extent that changes in climate might affect the variances of the former or the covariances contained within the latter, climate variability may be either reduced or increased as a result of the buildup of $\mathrm{CO}_{2}$ in the earth's atmosphere.

In the next section, the behavior of an agricultural firm faced with environmental uncertainty is considered. The factors that affect the firm's decision process as well as responses appropriate to uncertain climate information are discussed.

\subsubsection{Behavior of the Firm in the Face of Environmental Uncertainty}

In the absence of uncertainty, wealth maximization for the firm is relatively straightforward. A lack of uncertainty implies that the firm knows precisely the output quantity that it can sell at each price, the cost of producing each quantity, future values of exogenous variables that affect 
profits, etc. Under these conditions, the process of wealth maximization can be reduced to a mathematical exercise; no judgmental factors enter into the decision process.

When uncertainty becomes an intrinsic part of the problem, however, judgment may or may not enter into the decision process, depending upon whether the uncertainty can be precisely quantified. If one can measure the uncertainty with perfect accuracy--i.e., specify the true parameters of the underlying probability distributions--then the uncertainty can be treated within a probabilistic framework so that expected wealth is maximized. This is an objective expectation. On the other hand, if the true parameters of the underlying probability distribution are not known, but one has some sense of the nature of the underlying distribution, then expected wealth can still be maximized; however, the expectation in this case is subjective. (a)

Real world situations are invariably characterized by uncertainty that cannot be quantified precisely. Hence, judgments nearly always enter the decision process, and this is particularly true when decisions have to be made about events that are expected to occur some time in the distant future.

Figure 4.1 presents a flow diagram of a hypothetical agricultural firm's decision process under climatic uncertainty. The diagram shows that there is both uncertainty as to the character of the climate change as well as uncertainty regarding the predicted effects of the climatic change. It is assumed that climate change impacts can affect the wealth of the agricultural firm.

The obvious first step in the firm's decision process is a preliminary assessment of the credibility of both the climate change forecasts and their predicted impacts. This preliminary analysis may result in a determination that the information is not credible or that it is not relevant to any useful decision that the firm could make. In either case, no action would be taken

(a) It is appropriate to observe that while firms do not usually solve mathematical relationships in their attempts to maximize wealth, they do appear to engage in a decision process that produces results consistent with those obtained through mathematical optimization. In other words, the mathematical results generally agree closely with what is observed. 


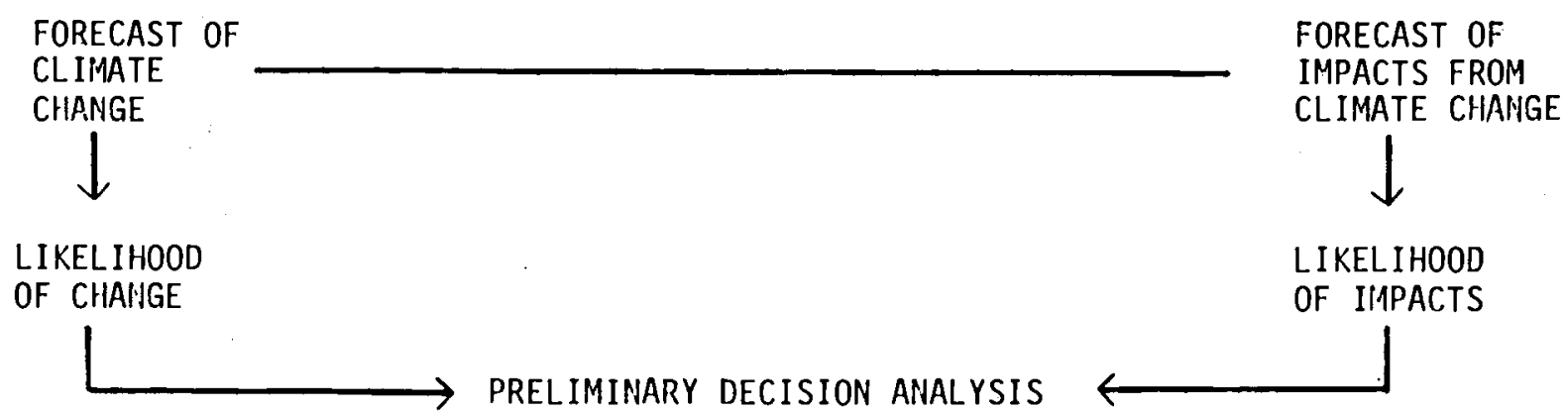

POSSIBLE CONCLUSIONS:

- infORMatiOn NOT CREDIBLE: tAKE NO ACTION

- INFORMATION NOT RELEVANT TO USEFUL DECISION: TAKE NO ACTION

- get adDitionAl INFORMATION

- DEFER DECISION UNTIL LATER PLANINING PERIOD

- CONDUCT MORE DETAILED DECISION ANALYSIS -

OTHER INPUTS TO DECISION PROCESS

- alternative actions that can be taken

- COST OF TAKING ALTERNATIVE ACTIONS

- RISK OF TAKING ALTERNATIVE ACTIONS

- BENIEFITS FROHI ALTERIJATIVE ACTIONS

- time fralie over which beliefits and costs acCRUE

- ACTIONS THAT COMPETITORS ARE TAKING
FORECAST OF

IMPACTS FROM

LIKELIHOOD

IIMPACTS 
on the basis of the information. Other options are to obtain additional information or to defer a decision until a later planning period. Finally, it may be decided to conduct a more detailed decision analysis. Factors Relevant to the Decision Process

If it is decided to undertake a detailed decision analysis, several other relevant factors need to be considered in order to determine whether some action is warranted and, if so, what kind. Factors that may be relevant to this process include:

- identification of the viable alternatives available;

- the cost of taking actions necessary to benefit from the information;

- the risk of taking alternative actions,

- the benefits from alternative actions;

- the time frame over which potential benefits (costs) from the information accrue; and

- whether others, especially competitors, are taking actions based on the information.

Identifying the Alternatives. The nature of the alternative actions that can be pursued under conditions of environmental uncertainty will depend importantly upon whether these actions involve a short-run adjustment to climatic variability or a long-run adjustment to climate change, which can include changes in climate variability. The distinction between the long- and the short-run is related to the fixity of certain inputs to production. In the short-run climate variability situation, economic agents are concerned with obtaining a more precise forecast of the initial value of specific meteorological variables, i.e., the amount of precipitation in July or the temperatures that can be expected in late August. Producers want this information so that they can decide what crops to plant, and when, and for planning their input purchases. Public and private irrigation authorities want this kind of information for planning water deliveries and reservoir operation, while input producers and suppliers can use this information to schedule production and inventories. Typically, these decisions on the part 
of firms in the agricultural sector are not related to actions that involve changing levels of capital stock, i.e., machinery and equipment purchases or construction of new facilities; nor do they generally involve substantial research and development investments. If these latter types of activities were sensitive to short-run climate information this would invariably mean substantial adjustments in capital stocks on a periodic basis. This is not observed because physical capital is usually "lumpy" and adjustments made in response to an expected change in, say, next month's rainfall cannot be reversed in order to take into account predicted changes in rainfall two months from now.

This is not to suggest that certain types of decisions are unaffected by environmental uncertainty. What is true is that, while investment in physical capital and research and development will not be sensitive to random fluctuations in meteorological variables, these types of decisions will be affected by climate change, whether this involves a real change in the expected values of meteorological variables or in the variances and covariances of their marginal or joint distributions. This means that, in looking to the future, only information that will impact on the ability of firms to make long-run adjustments in their capital stocks and research and development activities will be of value. Moreover, the value of any information that is developed in order to permit firms to better deal with long-run environmental uncertainty will be related to the time duration of the planning cycle that characterizes investments in physical capital and research and development (Howe 1980). Thus, if it only takes five years to develop a new seed variety, then planning decisions related to this activity will not be affected by changes that are expected to occur with a high degree of confidence in 50 to 100 years. Nor is it likely that better information about the nature of future changes in the environment will affect investment in machinery that depreciates very quickly.

Costs, Risks and Benefits of the Alternatives. Once the alternative actions that are available have been identified, it will be important for the decision-maker to determine the costs, risks and benefits associated with 
each. The greater the costs--and risks in the case of a risk-averse firm--relative to the benefits to be obtained, the less likely is it that the action will be taken.

Time Frame of the Costs and Benefits. For most activities, costs must be incurred before benefits can be obtained. Therefore, the costs are generally much more certain than the benefits. Furthermore, because benefits and costs should be discounted to the point in time at which the decision is made--i.e., when resources become comitted--the undiscounted benefits must often be many times larger than the undiscounted costs before the action can be justified.

To illustrate this latter concept, consider a decision that must be made today in order to avoid (obtain) a certain $\$ 100$ loss (gain) 50 years hence. Using a $3 \%$ real rate of discount, the maximum that a firm would be willing to spend would be $(1.03)^{-50} \times \$ 100$, or $\$ 20.80$. If the loss (gain) were deferred 100 years, the maximum would be only $\$ 5.20$. Instead of a certain loss in 50 years, let the risk of loss be $50 \%$. Then the firm might be willing to pay no more than $\$ 10$ to $\$ 12$ or $(1.03)^{-50} \times 0.5(\$ 100)$, to avoid or insure against this risk; the same risk of loss in 100 years would be worth no more than about three dollars. At rates of discount higher than three percent, all of these amounts would be even lower.

Risk-Averseness and Behavior of Competitors. Firms will weigh the risks of reacting to $\mathrm{CO}_{2}$ information against the risks of not reacting. These risks will be weighed against the expected pay-offs from acting and not acting. Firms that are risk-averse will be willing to sacrifice some expected income in order to reduce their risk by taking actions that will reduce the variance in the expected benefits. This will be illustrated in greater detail in Section 4.4 .2 .

If a firm's competitors respond to the information, the firm is likely to respond in a similar way. There are two reasons for this. First, most firms simply consider it too risky to stray too far from the rest of the pack. Second, if a 11 of one's competitors are responding one way to the same information, any decision analysis based on the same information which suggests a different course of action is more likely to be mistrusted. 
Possible Responses Based on the Decision Analysis

Once information of the type described above has been collected, the detailed decision analysis can be completed. Several possible results of the decision analyses are discussed below.

All Alternatives Are Too Costly. It may be determined that all of the actions available, except doing nothing, are too costly relative to the benefit to be obtained. It has just been illustrated how the discounting of future benefits might severely limit the actions that a firm would be willing to undertake during the current planning period.

Deferral of Decision. Closely related to the cost of taking an action is the length of time before a decision must be made. Costs are not incurred until resources are actually committed, so that if decisions can be deferred at little or no cost, then deferral of the decision will be the wise course. As noted earlier, the impacts from significant climate changes induced by increased concentrations of $\mathrm{CO}_{2}$ in the atmosphere are not generally expected to occur before the next fifty years. Given such a long lead time, one must consider carefully what decisions, if any, must be made within the current planning period.

When long lead times are available, it might well be possible at little or no cost to defer the decision to act. This possibility is illustrated in Figure 4.2. The solid curve shows the contemporaneous cost of preventing a $\$ 100$ loss due to an event that will occur in year $t_{50}$ with certainty. The cost is $\$ 25$ in year $t_{0}$, and it remains at this level through year $t_{25}$, at which time it begins to rise. As year $t_{49}$ approaches, the contemporaneous cost rises sharply and in year $t_{49}$ exceeds the $\$ 100$ loss. This curve characterizes the vast majority of situations, where the cost of completing some action increases (at an increasing rate) the shorter the period of time over which the action must be completed. The dotted curve in Figure 4.2 represents the present value of the solid curve, discounted at $3 \%$. This curve declines from $t_{0}$ until somewhat beyond year $t_{50}$, at which time it rises at an increasing rate. The minimum point on the dotted curve represents the 


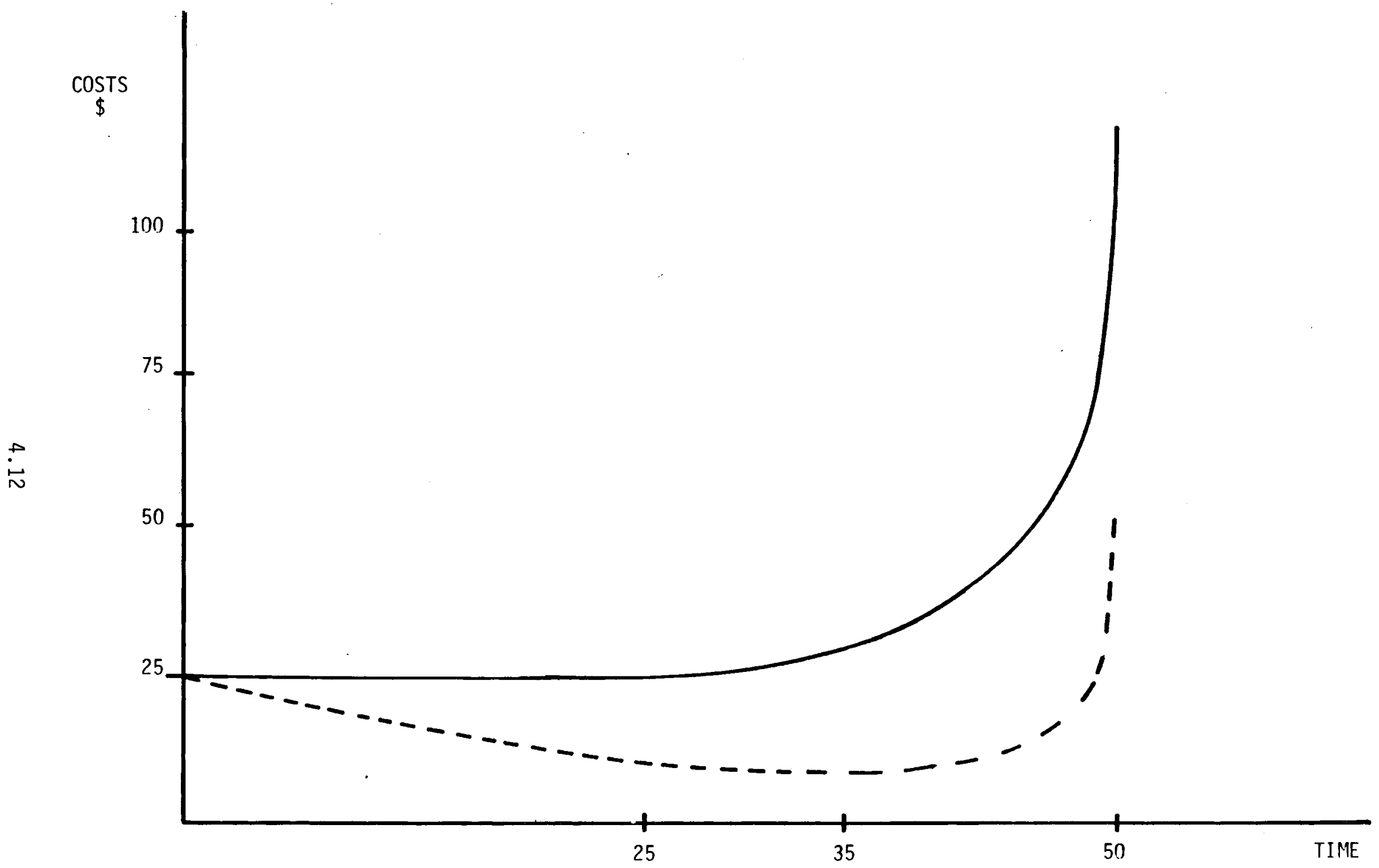

FIGURE 4.2. Relationship Between Cost and Time Remaining to Complete an Action 
optimal time, $t^{\star}$, to incur the cost necessary to prevent the $\$ 100$ loss. (At $t \star$ the contemporaneous costs are increasing at precisely the same rate as the rate of discount.)

Short-Term Response Pending Additional Information. Another possible conclusion of the decision analysis is to make a short-term response until better information becomes available upon which to make a long-term response. Deferral of action has already been discussed in connection with the factors that affect a firm's decision process, i.e., whether the decision can be postponed. Given that the firm decides to take some positive action, a short-run response might be typical when the decision is made under relatively uncertain conditions. The reason for this is that a long-run response often implies a long-term cormitment of significant capital resources. A mistaken decision can mean heavy losses. A decision to make short-run adjustments until at least some of the uncertainty can be resolved will often prove to be a wise course.

Consider the example illustrated hy the following loss table:

TABLE 4.1. LosS Table I

\begin{tabular}{crc}
\hline & \multicolumn{2}{c}{$\begin{array}{c}\text { State of } \\
\text { Environment }\end{array}$} \\
\hline $\begin{array}{l}\text { Action } \\
\text { Taken }\end{array}$ & A & B \\
\hline 1 & 0 & -175 \\
\hline 2 & -150 & -20 \\
\hline
\end{tabular}

State $A$ might correspond to no climate change, and State $B$ to an increase in aridity from global warming; Action 1 might be to do nothing, and Action 2 to install an irrigation system. Assume that the best available information indicates that $A$ and $B$ are equally probable. Then if some action must be selected now, losses are minimized by Action 2, with an expected loss of $\$ 85$ $(0.5 \times 150+0.5 \times 20)$, compared with $\$ 87.50(0.5 \times 0+0.5 \times 175)$ for 
Action 1. Assume further that this decision can be deferred for a year by taking some short-term action at, say, a net cost of $\$ 10$, and that some additional information is expected within the year that will indicate the true state of the world with $80 \%$ probability. If this new information indicates that State $A$ is $80 \%$ probable, the expected loss from choosing Action 1 is only $\$ 35(0.8 \times 0+0.2 \times 175)$; on the other hand, if the information indicates that State $B$ is $80 \%$ probable, the expected loss from choosing Action 2 is only $\$ 46(0.2 \times 150+0.8 \times 20)$.

In this example, the decision to make a short-term response is clearly superior: If State $A$ is 80 percent probable, the gain from waiting a year to make the long-run decision is $\$ 40$ (by reducing an expected loss of $\$ 85$ to an expected loss of $\$ 35$ plus the $\$ 10$ cost of the short-run response), while if State $B$ is 80 percent probable, the gain is $\$ 29$ (by reducing an expected loss of $\$ 85$ to an expected loss of $\$ 46$ plus the $\$ 10$ cost of the short-run response).

Obtain Additional Information. On the basis of a detailed analysis, a firm may also decide to acquire more information so that a better decision can be made. To illustrate this concept, consider Table 4.2, which shows the losses for each of seven actions associated with seven states of the world. For example, if the state of the world is $A$, then the best action is 1 ; this action minimizes losses at zero, while Action 2 gives losses of 10 , Action 3 losses of 20 , etc. The optimal action for State-of-the-Wor ld B is Action 2,

TABLE 4.2. Loss Table II

\begin{tabular}{|c|c|c|c|c|c|c|c|}
\hline \multirow{2}{*}{$\begin{array}{l}\text { Action } \\
\text { Taken } \\
\end{array}$} & \multicolumn{7}{|c|}{ State of the Wor Id } \\
\hline & $\bar{A}$ & $\bar{B}$ & $C$ & $D$ & $E$ & $\bar{F}$ & $G$ \\
\hline 1 & 0 & -10 & -20 & -30 & -40 & -50 & -60 \\
\hline 2 & -10 & 0 & -10 & -20 & -30 & -40 & -50 \\
\hline 3 & -20 & -10 & 0 & -10 & -20 & -30 & -40 \\
\hline 4 & -30 & -20 & -10 & 0 & -10 & -20 & -30 \\
\hline 5 & -40 & -30 & -20 & -10 & 0 & -10 & -20 \\
\hline 6 & -50 & -40 & -30 & -20 & -10 & 0 & -10 \\
\hline 7 & -60 & -50 & -40 & -30 & -20 & -10 & 0 \\
\hline
\end{tabular}


etc. Assume that the firm assigns the following (subjective) probabilities to each of the seven possible states of the world: $0.20,0.30,0.20,0.10,0.08$, 0.07 and 0.05 .

The respective expected payoffs for Actions 1 through 7 are: -19.7, $-13.7,-13.7,-17.7,-23.7,-31.3$, and -40.3 . For the subjective probabilities given, the expected losses to the firm are minimized with either Action 2 or 3, both of which have an expected loss of 13.7. Now, suppose that additional information is made available to the firm. This information causes it to revise its subjective probabilities to $0.18,0.40,0.19,0.09,0.06$, 0.05 and 0.03 . Then the expected payoffs for Actions 1 through 7 are now $-17.2,-10.8,-12.4,-17.8,-25.0,-33.4$ and -42.8 , respectively. Action 2 has the lowest expected loss, and the reduction in the expected loss from taking action 2 has dropped by 2.9 with the additional information.

From this analysis, it should not be concluded that the firm values the additional information at up to 2.9 units. First of all, if the firm would have taken Action 2 without the additional information, then there will be no real pay-off from the information. This is because the information resulted in a revision of the subjectively determined expected pay-off, and not the objectively determined pay-off; i.e., the real probabilities did not change. On the other hand, the additional information did reduce the risk perceived by the firm, and this has some value. In the next section, the question of the value of information will be taken up in more detail.

Other Responses. There are yet other ways in which a firm can respond to information that is characterized by uncertainty. These include:

- Altering output levels

- Altering output mix

- Altering mix of intermediate inputs

- Changing lever of investment

- Introducing flexibility into production function.

The first two of these responses will usually (though not always) involve short-run adjustments in the face of uncertainty due to climate variability, 
while the last two are more closely allied to long-run economic adjustments to climate changes. These responses are considered briefly in the following discussion.

Altering Output and Input Levels and Mix. A firm may decided to alter its level of output in response to uncertainty, although examples that seem plausible in the case of long-term climate change are not readily apparent. However, changes in the mix of agricultural inputs and outputs might be reasonable to expect. Pesticides, fertilizers, seeds and seedlings and other intermediate inputs might be altered if farmers became convinced that there was a reasonable chance that they were in the midst of a period of significant climate change, particularly if the climate change were characterized by an increase in the frequency of extreme weather conditions. Agricultural producers might also shift to different crops or types of livestock in order to take advantage of changing conditions. These kinds of decisions are all for the short-run, and are, therefore, not likely to be made until the effects of climate change are already apparent.

With regard to firms engaged in forestry, their decisions about where to plant new trees and what kinds of trees to plant might well be affected by climate changes that could impact yields in, say, 50 years. Trees, unlike many other agricultural products, have a very long growth cycle, and they can be profitably harvested well before they are fully matured. Consider the decision to plant a hardwood forest that, under current expectations, would be harvested in 70 years. Assume that the trees increase volumetrically at a constant annual rate of $6 \%$ through year 70 , when growth falls to a two percent rate, and that the impact of climate change would result in a harvest at the end of year 50. A tree worth $\$ 1000$ in year 50 would grow to a value of $\$ 3207$ in year 70. The present values of these amounts are $\$ 228$ and $\$ 405^{\circ}$, respectively. This example suggests that the returns may be quite sensitive to whether or not the predicted climate impacts do in fact take place and reduce the 1 ife of a forest by 20 years.

Adjusting Level of Investment. Levels of investment might be expected to change if significant agricultural impacts appear imminent as a result of 
climate change. Measures to protect crops and livestock from extremes of weather, and investments in irrigation systems to compensate for increased aridity are just two examples of investment decisions that might be made. A major avenue of investment lies in the inf ant genetic engineering industry, which apparently offers substantial potential for developing new strains of plants and animals that are more resistant to pests, diseases and extremes of weather.

Increasing Flexibility of Production Function. Introducing flexibility into the agricultural production function is another important way of accommodating climate change. Altering intermediate inputs and outputs and changing investment levels have already been discussed, but the production function can also be changed, for example, by lengthening or shortening the time between $p l a n t i n g$ and harvesting (the result of genetic engineering), and by using different types of capital equipment--for example, a machine that is better able to operate in temperature extremes, but which is somewhat less efficient at normal temperatures.

These then are the major types of responses by agricultural firms to climate-related information. In the following section, the value of information to the agricultural firm will be considered in greater detail.

\subsection{THE DEMAND FOR CLIMATE RELATED INFORMATION}

In this section, the demand for climate-related information is considered. The purpose here is two-fold: first, techniques for analyzing the decision process in a formal way will be considered; these techniques will therefore suggest how much decision-makers might be willing to pay for climate-related information. Second, this approach can potentially provide insights into the type of information that could be usefully provided and when it should be provided.

In evaluating information relating to the effects of increased atmospheric concentrations of $\mathrm{CO}_{2}$, the ir effects on climate, and the effects of climate on agricultural yields, a number of distinct elements need to be evaluated. For example, consider the situation in which a scientist, using 
historical data on changes in mean sea levels, reasons as follows: "Sea levels have been rising at an increasing rate. This is due to the melting of the west Antarctic ice sheet. The rate of melting has been increasing at an increasing rate because of secular global warming. The global warming is attributable primarily to increased atmospheric concentrations of $\mathrm{CO}_{2}$. (These concentrations have resulted largely from the burning of fossil fuels.) If (this rate of burning continues indefinitely into the future, and) the buildup of $\mathrm{CO}_{2}$ concentrations continue, certain specified global climate changes will occur, and these climate changes will result in certain specified impacts on agricultural yields." The portions of the argument in parentheses are not logically crucial to the conclusion.

Each of the steps in this reasoning process has some likelihood of being true, and the value of the information that certain impacts on agricultural yields will occur must be intimately tied to these likelihoods. The remainder of this section will focus on three topics:

- The use of Bayesian methods to derive the value for climate-related information and to provide a framework for evaluating research expenditures on long-range climate forecasting methods.

- The impact of uncertainty on the value of information to risk-averse firms.

- Market failures in the provision of long-range climate information.

\subsubsection{Bayesian Approach}

Bayesian analysis represents perhaps the most straightforward procedure for evaluating climate information. The following example illustrates the use of this approach to determine the value of climate forecasts in constructing alternative types of water resource supply facilities. A private irrigation authority, faced with the problem of how to best react to long-term climate changes, must decide what kind of storage and delivery systems to construct. The firm is able to characterize the expected states of the climate as: (1) no climate change, (2) moderate climate change, involving moderate declines in average monthly precipitation, and (3) severe climate change 
involving more dramatic decreases in average monthly precipitation and also greater seasonal variability in precipitation. In order to deal with these possibilities the irrigation company has three alternatives. They are:

1. keep the current system in tact, i.e., do nothing;

2. build a much larger capacity reservoir, but do nothing to change the current method of delivery; and

3. build a larger capacity system and install facilities that will permit real time metering and control of irrigation supplies at major junctions and individual points of final use in the system.

The pay-off table facing the irrigation company is shown in Table 4.3. Each column in this table indicates the annualized net benefits for the three alternatives given one of the three possible climate states. The prior probabilities, $P\left(S_{i}\right)$, in Table 4.3 are the firm's best assessment of the

TABLE 4.3. Pay-Off Table for Irrigation Authority

\begin{tabular}{|c|c|c|c|}
\hline & \multicolumn{3}{|c|}{ State of the Wor Id Environment } \\
\hline & $\begin{array}{l}\text { No Climate } \\
\text { Change }\end{array}$ & $\begin{array}{c}\text { Moderate } \\
\text { Climate Change }\end{array}$ & $\begin{array}{c}\text { Severe } \\
\text { Climate Change }\end{array}$ \\
\hline Prior Probability $P\left(S_{i}\right)$ & 0.3 & 0.4 & 0.3 \\
\hline
\end{tabular}

Action

Facility 1

$\$ 50,000$

$\$ 30,000$

$\$ 15,000$

Facility 2

$\$ 40,000$

$\$ 35,000$

$\$ 22,000$

Facility 3

$\$ 37,000$

$\$ 32,000$

$\$ 25,000$

likelihood of each climate outcome, given its current level of information. For example, the firm may have considered all of the evidence relating to $\mathrm{CO}_{2}$-inducing $\mathrm{climate}$ change in a separate decision analysis and on the basis of that analysis, decided that the probabilities were $0.3,0.4$ and 0.3 for no, moderate and severe climate change, respectively.

If the irrigation authority wants to pay no attention to uncertainty, which is irrational, it would select Facility 1 (i.e., do nothing) if it 
believed that no climate change was going to occur, Facility 2 if the expected climate change was to be moderate, and Facility 3 if it believed that the climate change would be severe. If it took into account only the prior probabilities in making this decision, then it would select the alternative for which has the highest expected annualized net value, or

$$
\operatorname{Maximize} \sum_{i} p_{i} v_{i j}
$$

where $P_{i}$ is the probability that climate state $i$ will occur and $V_{i j}$ represents the annualized value of alternative $j$ under climate state $i$. In this case, Facility 2 has the highest prior expected payoff of $\$ 32,600$, as opposed to $\$ 31,500$ for Facility 1 , and $\$ 31,400$ for Facility 3. [The payoff for Facility 2 is simply $0.3(\$ 40,000)+0.4(\$ 35,000)+0.3(\$ 22,000)=\$ 32,600$.]

Now, let $f_{j}(j=1, \ldots, 3)$ denote the three possible forecasts for climate change; namely, no change, moderate change and severe change. Let $s_{i}(i=1, \ldots, 3)$ denote the true future climate of the world. The application of this technique requires that the irrigation company be able to specify the likelihoods of receiving a climate forecast $f_{j}$, given that the true future climate of the world is $S_{i}$; i.e., it can specify $P\left(f_{j} / S_{j}\right)$, for all $i, j$. The matrix of values given by $P\left(f_{j} / S_{j}\right)$ is given in Table 4.4 below.

\begin{tabular}{|c|c|c|}
\hline $\mathrm{f}_{1-}$ & $\underline{f}_{2-}$ & $\underline{f}_{3}-$ \\
\hline 0.3 & 0.6 & 0.1 \\
\hline 0.2 & 0.3 & 0.5 \\
\hline 0.2 & 0.4 & 0.4 \\
\hline
\end{tabular}

Each cell entry in Table 4.4 gives the probability of receiving a forecast predicting the $j$ th climate state given that state $i$ actually occurred. If all forecasts were perfect, then each of the cells on the diagonal would be 1 , and all of the off-diagonal elements would be zero. 
Under usual circumstances, the likelihoods in Table 4.4 are obtained by sampling forecast information and determining what percent of the time each type of forecast is consistent with what actually occurs. This suggests that, even if forecasts are not sold in markets, their value can be determined. If the forecast is provided as a paid service (i.e., it is sold in a market) then the problem of sampling is more difficult because, in order to determine the likelihoods, the firm must purchase the information to do so. However, in practice, this problem is alleviated by advertising and marketing practices which are intended to provide perspective buyers with sufficient information to calculate the likelihoods. This does not impede the ability of firms that are making forecasts to sell them in the market because the actual forecast information that is to be purchased, if it is even available at the time the service is purchased, is not revealed.

Bayes' theorem states that

$$
P\left(S_{i} / f_{j}\right)=\left(P\left(s_{i}\right) P\left(f_{j} / S_{j}\right)\right) / \sum_{i}\left(s_{j}\right) P\left(f_{j} / S_{i}\right)
$$

namely, that each posterior probability is equal to the prior probability times the respective likelihood divided by a normalizing constant. Values for the numerator of the right-hand-side of (4.2) are given below in Table 4.5, along with the normalizing constant given by the corresponding row sum.

$$
\begin{aligned}
& \text { TABLE 4.5. } P\left(S_{j}\right) P\left(f_{j} / S_{j}\right) \\
& \begin{array}{lll}
0.09 & 0.18 & 0.03
\end{array} \\
& \begin{array}{lll}
0.08 & 0.12 & 0.20
\end{array} \\
& \text { Sum } \quad \frac{0.06}{0.23} \quad \frac{0.12}{0.42} \quad \frac{0.12}{0.35}=1.00
\end{aligned}
$$

The posterior probabilities are given in Table 4.6 below, and are simply the values in Table 4.5 divided by their respective column sums. 
TABLE 4.6. Posterior Probabilities

$\begin{array}{lccc}i=1 & \left.\frac{P(S}{i} \frac{/ f}{1}\right) & \left.\frac{P(S}{0} i \frac{f}{2} 2\right) & \left.\frac{P\left(S_{i} / f\right.}{0} \frac{f}{3}\right) \\ i=2 & 0.35 & 0.29 & 0.57 \\ i=3 & \frac{0.26}{1.00} & \frac{0.29}{1.00} & \frac{0.34}{1.00}\end{array}$

The irrigation company is now considering the purchase of a climate forecast. Such a forecast would enable it to make a decision based upon the posterior probabilities rather than the firm's prior probabllities. Should the firm purchase the forecast? The first step in answering this question is to calculate the expected pay-off from each of the three possible actions, first using the prior probabilities and then using the posterior probabilities associated with each of the three possible forecasts. In this case, the expected pay-off of building Facility 2 given no forecast has already been determined to be the highest of the three alternatives at $\$ 32,600$. However, once the posterior probabilities are applied in a manner consistent with Bayes' theorem, the situation changes. This is illustrated below in Table 4.7. The optimal strategy for each climate state is indicated by an asterisk (*). Now, the highest expected pay-off given a no change forecast is associated with the first type of facility (i.e., do nothing), the net annualized benefits from which are valued at $0.39(\$ 50,000)+0.35(\$ 30,000)+0.26(\$ 15,000)=\$ 33,900$.

TABLE 4.7. Expected Pay-Offs With and Without Climate Forecast

\begin{tabular}{|c|c|c|c|c|}
\hline \multirow[b]{3}{*}{ Action } & \multicolumn{4}{|c|}{ Expected Pay-0ff } \\
\hline & & & orecast 0 & \\
\hline & $\begin{array}{c}\text { No } \\
\text { Forecast }\end{array}$ & $\begin{array}{c}\text { No } \\
\text { Change }\end{array}$ & $\begin{array}{c}\text { Moderate } \\
\text { Change }\end{array}$ & $\begin{array}{l}\text { Severe } \\
\text { Change }\end{array}$ \\
\hline$\overline{F a c i l i t y ~} 1$ & $\$ 31,500$ & $\$ 33,900 *$ & $\$ 34,050 *$ & $\$ 26,750$ \\
\hline Facility 2 & $\$ 32,600 *$ & $\$ 33,570$ & $\$ 33,330$ & $\$ 31,030 *$ \\
\hline Facility 3 & $\$ 31,400$ & $\$ 32,130$ & $\$ 32,070$ & $\$ 30,070$ \\
\hline
\end{tabular}

* = Optimal Strategy 
The optimal actions for the two remaining climate states are to do nothing in the event of a forecast for moderate $\mathrm{climate}$ change, and to construct Facility 2 in the event of a forecast for severe climate changes. Notice that, the third facility is not optimal under any forecast conditions.

Since the forecast is not known until it is purchased, the expected value of the forecast must be computed. This is simply the weighted sum of the optimal forecast strategies, where the weights are the probabilities of receiving each of the three forecasts. These weights were calculated earlier as $0.23,0.42$ and 0.35 . The expected value of the forecast is $0.23(\$ 33,900)+$ $0.42(\$ 34,050)+0.35(\$ 31,030)=\$ 32,960$. Without the forecast the optima 1 strategy is to construct Facility 2 with an expected pay-off of $\$ 32,600$. The difference between the two amounts is $\$ 32,960-\$ 32,600=\$ 360$, which represents the agricultural value of the forecast information. Thus, if the 1 ife of the project was assumed to be 50 years, then the irrigation company would be willing to pay up to $\$ 18,000$ for the climate forecast.

A major weakness with the above approach is the requirement that the firm be able to specify the probabilities $P\left(f_{j} / S_{j}\right)$. In short-run situations this is not an unduly harsh requirement because producers can compare the accuracy of available forecasts with the actual states of nature that occur and establish the likelihoods $P\left(f_{j} / S_{j}\right)$ fairly readily. Doing so amounts to little more than being able to say, with a fairly high degree of confidence, that the weatherman is right about $x$ percent of the time when it rains. In the case of long-run climate forecasts, on the other hand, establishing the necessary likelihoods is far more complicated, for essentially two reasons. The first is that there are not comparable forecasts on which to judge their accuracy. The second problem is that by the time one is able to make an assessment of the accuracy of any such forecast, the reason for providing the forecast in the first place will not longer be valid: the climate will have already changed and the value of the information contained in the forecast will be of little use. Therefore, it is questionable whether a firm would, in fact, be able to supply the likelihoods, such as those appearing in Table 4.4, that are necessary to implement this techniques. 
However, the likelihoods expressed in Table 4.4 could just as easily reflect information that was developed as a part of a climate research program. For example, given information about past climatic states, different from those today, researchers could then develop models to predict, on an ex post basis, what the climate was like during these periods. By comparing the accuracy of these ex post forecasts with what is actually known, it would then be possible to develop a set of likelihoods such as those shown in Table 4.4. The same models could also be used to make ex ante forecasts of future climate states in order to provide prior probabilities such as shown in the original pay-off matrix, Table 4.3. With this information in hand, it would be possible to work through the entire exercise and calculate the expected value of the forecast from the perspective of the research agency. In that context, the $\$ 32,600$ figure in the previous example would represent the value of a research agency forecast without regard to the information it had obtained about the accuracy of the forecasting instrument. The $\$ 32,960$ figure would represent the expected value of a forecast given the knowledge about the accuracy of the forecasting method. The expected gross value of the forecast would be the difference between the two figures-- $\$ 360$ annually and $\$ 18,000$ over the life of the project--assuming, of course, that the original cost benefit study used to analyze the analysis was correctly performed.

Whether or not research in climate forecasting should be continued in order to improve the accuracy of climate forecasts in this situation will depend not on the expected gross value of the forecast, but rather upon the expected net value of the forecast, after taking into account the research and development costs required to provide the forecast and determine its accuracy. In order to conduct such an analysis, the following elements are required:

1. A set of regional forecasts of the expected economic impacts of $\mathrm{CO}_{2}$ buildup.

2. An analysis of the potential of key industries to take actions that would enable them to lessen the adverse impacts and take advantage of the beneficial effects of $\mathrm{CO}_{2}$ buildup. 
3. A prior distribution describing the currently known probabilities of different states of the environment that will impact upon these firms in the future.

4. A set of likelihoods, developed through research, that express the likelihood of receiving a forecast $f_{j}$, given that the true state of the climate will be $S_{i}$.

5. The costs of developing the forecasting instrument and conducting the economic analysis.

Using (1) and (2) it would be possible to develop an initial pay-off table showing the net benefits associated with specific industry plans to adjust to the impacts created by $\mathrm{CO}_{2}$ buildup. For example, assume that an initial economic impact analysis showed substantial declines in net societal benefits due to adverse impacts on corn yields in the corn belt states. One could then obtain information from plant breeders about expected future yield changes anticipated as a result of alternative research and development plans. These new yield values would be inserted into an agricultural sector mode 1 to show the resulting impacts of higher corn yields on net societal benefits for different climate states. The changes in net benefits, less private research and development costs, could then be used to construct a pay-off table for this industry. Elements (3) and (4), which constitute the prior probabilities and the likelihoods, respectively, could be manipulated to produce an expected pay-off table, such as Table 4.6, and this information could be employed to determine the expected value of a state-of-the-art climate forecast to $\mathrm{plant}$ breeders. The process would then be repeated for other key industries, such as forestry, and federal water resources development agencies in order to arrive at an estimate for the total expected value of the forecast information. If the difference between the expected value of the forecast and the costs of developing the forecasting technology and conducting the economic analysis is positive then the investment will have been an efficient one.

At first it may appear that the method above has an obvious flaw, namely that it represents a form of ex post analysis. That is, the efficiency of the 
research investment is analyzed after the investment is made, rather than before hand. While this is true, it can be argued that, once the value of an early stage of model development is determined, the difference between the expected benefits of an initial forecast and all research costs represents the expected net marginal rate of return on this initial investment. If the difference is positive, then this would be an indication that there is under-investment in this type of forecasting technology and that society could be still better off by investing more money in this area. In that framework, the decision rule for making further investments in this technology would be to invest until the incremental change in expected net value of the forecast is just equal to the incremental research costs. Thus, large positive differences between expected value of the forecast and research costs would be an indication that further investment is justified. As the difference declines (and it should if only because better forecasting methods will reduce the size of the off-diagonal probabilities in the likelihood matrix), this will be an indication that the level of expenditure ought to be decreased, incrementally until the expected marginal rate of return on research in this area is zero.

Such an approach necessarily implies that investments in the provision of climate information and economic forecasts should be provided on an iterative basis. This is in accordance with adoptive learning processes (Rausser and Hochman 1979). At the outset of such a process there is a great deal. of uncertainty surrounding the nature and timing of future climate changes and the economic consequences of these changes. Available information is utilized initially, in the manner described above, to allocate public research dollars to the provision of better information with the objective of maximizing the net economic return on these investments to society as a whole. The economic benefits of this information to decision makers in the public sector can be evaluated in terms of the economic costs foregone through the imposition of regulatory controls on the burning of fossil fuels. The private benefits of this information (in lieu of regulation) can be valued in terms of the opportunities it provides to key industries to take actions that will permit them to forego some of the costs associated with adjusting to the forecast climate changes before they actually occur. 
Once the initial reseach is completed, the new information is evaluated and the process described above is repeated, iteratively, until (and if) a position is reached where the value of additional information to society is equal to the additional costs of providing that information. In that situation (which is really not characteristic of non-stationary processes such as climate change), an optimal amount of learning and, by implication, an optimal degree of adaptation to climate change will have been achieved.

\subsubsection{Risk Aversion and the Value of Information}

One of the problems with the use of Bayesian methods to determine the value information is that they do not ordinarily take into account the impact of uncertainty on the willingness of risk-averse firms to pay for information that reduces uncertainty about future states of the environment.

In order to illustrate the effect that risk-averse behavior on the part of firms will have on their willingness to pay for research that reduces the amount of uncertainty regarding future changes in the state of the environment, consider the following problem. To simplify the analysis, assume that future changes in the enviroment will be sudden; that firms know when these changes will occur; and that the only uncertainty that exists is about the magnitude of these changes. While the first two assumptions are inconsistent with current knowledge about $\mathrm{CO}_{2}$ buildup, introducing less stringent assumptions into the analysis requires a level of mathematical sophistication that tends to obscure rather than illuminate the major point of this exercise.

Given this set of assumptions, the present value of a firms' expected utilty at the time these changes occur, or $\operatorname{PVE}\left(U_{t}\right)$, can be expressed as

$$
\operatorname{PVE}\left(U_{t}\right)=(1+r)^{-t} U\left[E\left(B_{t}\right), \sigma_{B}^{2}\right]
$$

where $(1+r)^{-t}$ is the discounting factor used to convert the future value of expected utility, $E\left(U_{t}\right)$, into its current worth; $E\left(B_{t}\right)$ represents the expected net benefits from production (i.e., profits) in period $t$ conditional upon the expected state of the environment; and $\sigma_{B}^{2}$ represents the variance in 
net benefits associated with current uncertainties about the magnitude of changes in the environment. Thus, expected utility in any given period is a function of expected benefits and the variance in the distribution of benefits. The partial derivatives of the expected utility function $U[]$ with respect to its arguments are

$$
\frac{\partial U}{\partial E\left(B_{t}\right)}>0 \quad \frac{\partial U}{\partial \sigma_{B}^{2}} \leq 0
$$

Thus, expected utility will increase when expected benefits rise, while an increase in the uncertainty of net benefits, as measured by $\sigma_{B}^{2}$, will cause expected utility to decline if firms are risk averse. If firms are risk neutral in the ir preferences, changes in $\sigma_{B}^{2}$ will have no effect on expected utility. This represents the classical distinction between risk-averse and risk-neutra 1 preferences.

In keeping with earlier statements, note that as $t$ increases the present value of expected utility will decline, so that if $t$ is a long way off in the future PVE $\left(U_{t}\right)$ will be very small. However, assume that by spending money in the current period, $t=0$, it is possible to reduce the uncertainty associated with our knowledge about the magnitude of environmental changes. The cost of research can be defined as

$$
C=C\left(R_{0}\right)
$$

where $R_{0}$ represents research effort in the current period. The relationship between uncertainty and research effort can be written in general form as

$$
\sigma_{B}^{2}=f\left(R_{0}\right)
$$

where $\partial f / \partial R_{0}<0$ to reflect the fact that additional research effort will reduce the variance of net benefits associated with the uncertainty about the magnitude of future environmental changes. 
There are at least two very stringent assumptions built into Equation (4.5). The first is that additional research effort will shed no new light on the expected future state of the environment, which will directly affect expected future benefits. While Equation (4.5) could have been written differently to reflect this possibility, the real problem with this formulation is that it assumes that the relationship between uncertainty and research effort is known, a priori. However, if this were the case then no research would have to be conducted in order to reduce the uncertainty that currently exists. Thus, the model developed here is not in the form of an ex ante planning model. That is, it cannot be used to determine how much money ought to be invested in research. However, that is not the purpose of this exercise. Rather, the objective of this discussion is to show why, from an analytical standpoint, risk-averse economic agents will place a value on information that reduces the amount of uncertainty associated with expected $\mathrm{CO}_{2}$-induced climates even though that information may have no effect on the expected value of benefits. And, while it is true that this same point could be illustrated by using stochastic control formulations (Rausser and Hochman 1979; Porter 1975; Cropper 1975), the necessary mathematical development to this is much more obtruse. A simplier model illustrates the general issue well enough.

Given Equations (4.3), (4.4) and (4.5) one can form the problem

$$
\text { Maximize } Z=(1+r)^{-t} U\left[E\left(B_{t}\right), \sigma_{B}^{2}\right]-C\left(R_{0}\right)
$$

subject to

$$
\sigma_{B}^{2}=f\left(R_{0}\right)
$$

where the objective function is measured as the difference between the present value of expected utility in period $t$ and current research costs. By substituting $f\left(R_{0}\right)$ from Equation (4.7) for $\sigma_{B}^{2}$ in the objective function and taking the partial derivative of $Z$ with respect to $R_{0}$, one obtains the single first-order condition for this model. 


$$
\frac{\partial Z}{\partial R_{0}}=(1+r)^{-t} \frac{\partial U}{\partial f} \frac{\partial f}{\partial R_{0}}-\frac{\partial C}{\partial R_{0}}=0
$$

This condition requires that in order for an optimum to be achieved, investments in research in the current period should be undertaken until the increase in the present value of expected utility due to the last unit of research effort is just equal to be marginal cost of research. Holding the discount rate constant, the change in the present value of expected utility due to a one unit increase in $R_{0}$ is the product of two terms. The first of these terms, $\partial U / \partial f$, represents the change in $U$ due to reduced uncertainty, and is a measure of risk aversion. The second term, $\partial f / \partial R_{0}$, represents the rate of change in uncertainty due to increased research effort. Since both of these terms are negative by definition, the entire expression for the change in the present value of expected utility due an additional unit of research effort, or

$$
\frac{\partial \operatorname{PVE}\left(U_{t}\right)}{\partial R_{0}}=(1+r)^{-t} \frac{\partial U}{\partial f} \frac{\partial f}{\partial R_{0}}
$$

will be represented by a downward sloping demand curve for research. This implies directly that

$$
\frac{\partial \operatorname{PVE}\left(U_{t}\right)}{\partial R_{0}} \geq 0 \quad \frac{\partial^{2} \operatorname{PVE}\left(U_{t}\right)}{\partial R_{0}{ }^{2}}<0
$$

The supply curve for research is upward sloping, since $\partial C / \partial R_{0}>0$, consistent with increasing marginal costs. The optimal amount of this type of research is then determined by the intersection of the supply and demand curves where

$$
\frac{\partial \operatorname{PVE}\left(U_{t}\right)}{\partial R_{0}}=\frac{\partial C}{\partial R_{0}}
$$


From this simple problem, it can be concluded that:

1. Holding other things constant, the more risk averse firms are the more they will be willing to spend on research that reduces uncertainty about the magnitude of $\mathrm{CO}_{2}$-induced environmental changes, even though that information has no effect on expected benefits.

2. The larger the amount of uncertainty about the magnitude of $\mathrm{CO}_{2}$-induced environmental changes, holding other factors constant, the more risk averse firms will be willing to spend for research that reduces that uncertainty.

3. The higher the productivity of research in reducing uncertainty, other things being equal, the more risk averse firms will be willing to pay for this research.

4. Any of the above effects will to some degree offset the influence which either high discount rates or very distant future impacts have on the value of the information provided by research.

\subsubsection{Market Failure and the Value of Information}

The thrust of the two previous sections has been to show that information which reduces the uncertainty about the future state of the environment can be priced. One conclusion that could be drawn from this is that since long-range climate forecasts will be of private value to certain types of firms in the agricultural sector, private markets for this information will come into existence. A corollary to this conclusion is that if private markets for this information do not materialize, then it is because the marginal private cost of producing this information is always above the private marginal value of that information to firms. The likely reason for this could be attributed to the "fact" that the present value of this information is very low due to discounting and to the possibility that very few firms conduct activities which require a 50 to 100 year planning horizon. Based on this line of reasoning one might ultimately conclude that, for these reasons, government sponsored research in this area represents an inefficient allocation of resources. However, it is also possible to draw a second conclusion, namely 
that because of certain characteristics of the information being provided, a competitive market will under-invest in long-range climate forecasts and that intervention by the public sector is required in order to provide a more efficient allocation of resources in this area. The remainder of this section will be devoted to a discussion of the characteristics inherent in the information contained in long-range climate forecasts that supports the latter of the two general conclusions outlined above.

\section{Climate Information as a Public Good}

Any kind of climate information must be characterized as a public good. A public good can be defined, formally, as a good whose consumption by one economic agent is not affected by another economic agent. This simply means that if $x$ amount of such a good is made available to say, two firms, then both of them can consume $x$ quantity of the good, whereas in the case of a private good, if one firm consumed $x$ amount of the good the most that the second firm would be able to consume would be $x-x_{1}$. Based on this definition, climate forecasts can be characterized as public goods since two firms can simultaneously use the same information without mutually affecting the amount of information available to either one. However, the public good characteristics of climate forecasts does not mean that they cannot be provided in private markets. They can. However, in order to do this, it must be possible to exclude some firms from using the climate information while making it available to others. If this is possible, then the information can be produced and sold in a private market. But even if this can be done, it can still be shown that the private firm will not supply the optimal amount of information, unless it can behave like a perfectly discriminating monopolist in its pricing practices (Samuelson 1955).

\section{The Free-Rider Problem}

However, once the climate information is produced and sold to another firm, the purchaser can destroy the monopoly by reproducing it at a very low cost and selling it to other firms which can, in turn, try to sell the information to other firms, etc. This gives rise to the classic free-rider problem described by Arrow (1962). Because information can usually be 
reproduced at a small fraction of the cost to produce it, the monopolist loses $h$ is advantage and the private incentive to produce the information is weakened. Hirschleiffer (1971) has argued that this need not be the case if the information conveys a market advantage to the purchaser. In that case, the purchaser has every incentive not to reproduce the information and the problem of free-ridership is eliminated. However, this will only be true when it is possible to exclude firms which are unwilling to pay for the information from purchasing it.

In the case of climate information, it may be contractually possible to exclude some firms from obtaining information, free, that is sold to other firms. On the other hand, this may be difficult to do in practice since excluded firms can observe the behavior of firms who have purchased the information and infer the content of the forecast based on these observations. If this can be done, then the problem of free-ridership is not eliminated and the potential producer of the information will have little incentive to produce because he will not be adequately compensated for his effort. Under these conditions it will be optimal for everyone to wait unt il someone else produces this information. Thus, differences between the cost of producing and reproducing climate information, along with the problem of excludability suggest that private markets for long-range climate information may not come into being even though the information might be very valuable. Even if such markets do spring up, then the same free-rider problem may lead to under-investment in whatever amount of long-range climate information is provided.

The problems associated with free ridership in markets for information can be reduced to some extent by legally protecting firms which produce the information from other firms which could reproduce the information at low cost and sell it for their own private gain. This is the underlying rationale for patent and copyright laws which make it easier for firms to appropriate a larger share of the benefits that are derived from information. Thus, potential producers of long-range climate information may be protected to some degree by the problems posed by free ridership. However, even if this protection were complete (which it is not) there may be a more basic reason for 
not trusting private markets to make socially optimal investments in long-range climate information. Since much of the research that will be required to provide accurate long-range climate forecasts will fall under the heading of basic research, legal restrictions on the transmittal of research findings are likely to reduce the efficiency of inventive activity and therefore reduce its quantity and quality, as well.

Market failure in the provision of long-range climate information would mean that, left in the hands of private markets, the potential benefits of providing this information would not be fully realized. The justification for the public provision of weather information, which is clearly of considerable private value, can be found in the potential for market failures to inhibit the production of this information by private firms. With the exception of highly specialized information that would be of direct benefit to a very small number of firms, it seems likely that market failure would pose a similar problem in relying upon private markets to produce a socially optimal level of information about $\mathrm{CO}_{2}$-induced $\mathrm{climate}$ change. 


\section{REFERENCES}

Arrow, K. J. 1962. "Economic Welfare and the Allocation of Resources for Invention." In The Rate and Direction of Inventive Activity: Economic and Social Factors, pp. 609-626. National Bureau of Economic Research Conference Series, Princeton, New Jersey.

Chen, K. et al. 1980. "Carbon Dioxide from Fossil Fuels." Energy Policy. December 1980, pp. 318-330.

CIAP. 1975. Economic and Social Measures of Biologic and Climate Change. CIAP Monograph 6, Department of Transportation Climatic Impact Assessment Program. DOT-TST-T5-56, National Technical Information Center, Springfield, Virginia.

Council on Environmental Quality. 1981. Global Energy Futures and the Carbon Dioxide Problem. U.S. Government Printing Office, Washington, D.C.

Cropper, M. L. 1975. "Optimal Sequential and Nonsequential Strategies for Stratospheric Flight." In Economic and Social Measures of Biologic and Climate Change. CIAP, U.S. Department of Transportation, pp. 2.51-2.63. DOT-TST-75-75, National Technical Information Service, Springfield, Virginia.

Hansen, J. et al. 1981. "Climate Impact of Increasing Atmospheric Carbon Dioxide." Science 213, pp. 957-996.

Hirschleiffer, J. 1971. "The Private and Social Value of Information and the Reward to Inventive Activity." American Economic Review 61(4):561-574.

Howe, C. W. 1980. "An Overview of Conceptual and Methodological Issues Arising in Climate Impact Assessment and Policy Formulation." Paper presented at the National Climate Program Office Workshop on the Methodology for Economic Impact Analys is for Climate Variation and Change, April 24-25, 1980, Fort Lauderdale, Florida.

Kellog, W. W. and R. Schware. 1981. Climate Change and Society, Consequences of Increasing Atmospheric Carbon Dioxide. Westview Press, Boulder, Colorado.

National Research Council. 1979. Carbon Dioxide and Climate: A.Scientific Assessment. National Academy of Sciences, Washington, D.C.

Porter, W. R. 1975. "Sequential Methods of Information Collection and Monitoring for Stratapheric Flight." In Economic and Social Measures of Biologic and $\mathrm{Cl}$ imate Change. CIAP, U.S. Department of Transportation, pp. 2.65-2.72. DOT-TST-75-56, National Technical Information Center, Springfield, Virginia.

Rausser, G. C. and E. Hochman. 1979. Dynamic Agricultural Systems: Economic Prediction and Control. North Holland, Amsterdam, The Netherlands. 
Samuelson, P. A. 1955. "Diagrammatic Exposition of a Theory of Public Expenditure. Review of Economics and Statistics $37(4): 550-556$.

Taylor, J. B. 1976. "Methods of Efficient Parameter Estimation in Control Problems." Annals of Economic and Social Measurement 5(5):339-348.

Zellner, A. and V. K. Chetty. 1965. "Prediction and Decision Problems in Regression Models from the Bayesian Point of View." Journal of the American Statistical Association 60(4):608-616.

Zellner, A. and M. S. Geisel. 1968. "Sensitivity of Control to Uncertainty and the Form of the Criterion Function." In The Future of Statistics. ed. D. J. Watts, Academic Press, New York, New York. 


\subsection{ANALYSIS OF METHODS FOR MODELING YIELD RESPONSE $\mathrm{TO}_{2} \mathrm{CO}_{2}$-INDUCED ENVIRONMENTAL CHANGE}

Chapters 3.0 and 4.0 of this report were intended to deal with general theoretical problems: to provide a framework, first of all, for understanding how $\mathrm{CO}_{2}$-induced environmental changes can work from the agricultural sector through the rest of the economy and, secondly, for linking impact analys is to public and private decisions that inevitably will be made in the future to adjust to these changes. This chapter initiates the formal analysis within the report of specific types of models that can be used to conduct future studies of the impact of $\mathrm{CO}_{2}$-induced environmental changes within and beyond the agricultural sector of the U.S. economy. In that context, the selection of crop modeling as the topic to begin the analysis is hardly accidental. Without exception, all of the paths of $\mathrm{CO}_{2}$-induced environmental change identified in Chapter 3.0 can be traced forward until they ultimately lead to crop yields. Consequently, before one can even begin to model the economic impacts of future increases in the atmospheric concentration of $\mathrm{CO}_{2}$, a systematic effort must be undertaken to translate the impacts of $\mathrm{CO}_{2}$ on the environment to changes in actual or potential crop yields.

This chapter will be divided into two main sections. The first section will examine some of the methodological problems associated with integrating information about the foreseeable impacts of increases in ambient $\mathrm{CO}_{2}$ into yield prediction models. . The second section will outline the different types of methods that can be used to predict the effects of $\mathrm{CO}_{2}$-induced environmental changes on crop yields, and then evaluate the comparative strengths and weaknesses of these different approaches using specific examples drawn from the literature in the field of crop modeling.

\subsection{METHODOLOGICAL ISSUES IN FORECASTING CO 2 -INDUCED CROP YIELD CHANGES}

Modeling the economic impacts of $\mathrm{CO}_{2}$-induced environmental changes can best be understood as a three-stage process. In the initial stage, climate and global circulation models are employed in conjunction with other pieces of 
information to make forecasts about the impacts of hypothetical increases in the ambient concentration of $\mathrm{CO}_{2}$ on regional climates. In the second stage, information about the expected concentrations of $\mathrm{CO}_{2}$ and their impact on the parameters of the distributions of regional meteorological variables are used in conjunction with pest and crop yield models to generate forecasts about the first- and second-order effects of $\mathrm{CO}_{2}$ on crop yields. Finally, this information on expected crop yields is used to drive economic models of the agricultural sector and the rest of the economy in order to provide the necessary forecasts of the direct and indirect economic impacts associated with different yields.

There are two major problems with this approach that must be dealt with on a more systematic basis if it is to be used successfully in the manner suggested in the previous chapter. The first of these problems relates to the generation of regional climate forecasts--specifically to the type of climate information that is required not only to drive crop yield models, but also to provide yield forecasts in a framework that is consistent with the randomness of meteorological events. The second problem relates to the independence of the first- and second-order effects of $\mathrm{CO}_{2}$ on crop yields from the management decisions of producers.

\subsubsection{Climate Forecasts and Yield Prediction}

The substance of the meteorological information that will be required to generate forecasts of the effects of $\mathrm{CO}_{2}$ on crop yields involves two considerations. The first of these relates to the input requirements of the different kinds of models used to produce yield estimates. In some cases, information on the daily values of solar radiation, precipitation and temperature, by location, is required to produce annual yield estimates, while other models may only need the monthly values of selected meteorological variables, such as precipitation or temperature, or composite variables, such as potential evapotranspiration or growing degree days, in order to provide crop-spec if ic yield forecasts on an annual basis. The input requirements of the different types of yield models will be discussed in much greater detail in the next section of this chapter. In general, however, the $\mathrm{fit}$ between the 
input demands of even the least sophisticated of these models and the meteorological information provided by general circulation model experiments is not very good.

In most cases, the available climate forecasts that have been generated by these experiments yield information about annual, and in at least one case (Wetherald and Manabe 1981) monthly changes in the mean values of temperature, precipitation and soil moisture by latitude only, over an idealized geographic domain. The lack of congruity between this information and the impact requirements of crop yield models has already been touched upon in a somewhat different context in the first section of Chapter 3.0. There, the argument was advanced that building climatological scenarios on an eclectic basis, using information from a wide variety of sources, may be more valuable to decision-makers in both the public and private sectors in terms of helping them adjust to the future than waiting until the state-of-the-art in global circulation modeling has progressed sufficiently. In that general context, mating the information that is contained in these scenarios to specific crop yield models is an extremely important technical consideration.

However, an equally important consideration in developing the necessary climatological information to predict crop yields involves the need to adopt a framework for this information that is consistent with the stochastic, or random, nature of meteorological events. In general terms, this means that the concept of $\mathrm{CO}_{2}$-induced climate change which is embodied in the substance of climate scenarios or forecasts must in some way address the issue of climate variability as well as climate change.

Introducing the dimension of climate variability into climate scenarios is important for two reasons. The first of these is primarily a statistical phenomenon and relates to the impact of climate variability on the calculation of the expected (average) value of crop yield. The second issue involves the effect that year-to-year fluctuations in crop yields have upon the risks which agricultural producers assume when they decide to plant certain crops in a region. 
The effect of climate variability on the calculation of the expected value of crop yields can best be illustrated by examining a relatively simple model which relates crop yield to specific values of relevant meteorological variables. Assume that the yield of a particular crop can be expressed as a function of meteorological variables in the following terms:

$$
Y=\beta_{0}+\sum_{i=1}^{m} \beta_{i} W_{i}+\frac{1}{2} \sum_{i=1}^{m} \sum_{j=1}^{m} \beta_{i j} w_{i} w_{j}
$$

Where $Y$ stands for yield; $W_{j}$ is the value of the ith meteorological variable in the mode 1 for $i, j=1, \ldots, m$; and $\beta_{0}, \beta_{i}$ and $\beta_{i j}$ are parameters which "explain" the relationship between crop yield and the values of the relevant meteorological variables.

The meteorological variables in the mode 1 are assumed to be random. The probability that a particular set of meteorological events, $W_{t}=\left(W_{1}, \ldots\right.$, $W_{m}$ ) will occur at a given location in year $t$ can be expressed as

$$
P\left(\bar{W}_{1}, \ldots, \bar{W}_{m}\right)=g\left(\bar{W}_{1}, \ldots, \bar{W}_{m}\right)
$$

where the function of $g()$ represents the joint distribution of meteorological variables, based on the observed historical pattern of meteorological events at a specific location. Given the vector $w_{t}$, the model shown in Equation (5.1) could be used to predict the corresponding yields that would be achieved, consistent with the values contained in the vector, $w_{t}$. The expected value of yield can be expressed as

$$
\begin{aligned}
& E\left[f\left(W_{1}, \ldots, W_{m}\right)\right]=E(Y) \\
& =\int_{-\infty}^{\infty} \ldots \int_{-\infty}^{\infty} f\left(W_{1}, \ldots, w_{m}\right) g\left(W_{1}, \ldots, W_{m}\right) d W_{1}, \ldots, d w_{m}
\end{aligned}
$$


where $f()$ is the crop yield function from Equation (5.1) and $g()$ is the joint distribution function from Equation (5.2).

The exact distributions of combinations of random variables are not easily developed; however, the parameters of these distributions are generally more accessible. In the current example, the algebra of expectations can be readily applied to Equation (5.1) in order to obtain the expected value of yield for that model, which is

$$
\begin{aligned}
E(Y) & =\beta_{0}+\sum_{i=1}^{m} \beta_{i} E\left(W_{i}\right)+\frac{1}{2} \sum_{i=1}^{m}\left[\beta_{i j} E\left(W_{i}\right)^{2}+\sigma_{i}{ }^{2}\right] \\
& +\frac{1}{2} \sum_{i \neq j}^{m} \sum_{j}^{m} \beta_{i j}\left[E\left(W_{i}\right) E\left(W_{j}\right)+\operatorname{CovaR}\left(w_{i}, w_{j}\right)\right]
\end{aligned}
$$

where $E\left(W_{j}\right)$ and $\sigma_{i}{ }^{2}$ represent the expected value and the variance, respectively, of the ith meteorological variable, and COVAR $\left(w_{i}, w_{j}\right)$ is the covariance between $w_{i}$ and $w_{j}$. Computationally, the covariance term is equivalent to the product of the standard deviations of $w_{j}$ and $w_{j}$ times the correlation coefficient between the two random variables. If any pair of meteorological variables are statistically independent, the correlation coefficient between them will be zero and the covariance term in Equation (5.4) will drop out for that pair of variables.

Equation (5.4) illustrates that in this particular case the expectation of crop yield is not equal to Equation (5.1) evaluated at the expected values of the meteorological variables in the model. Rather, the computation of expected yield involves both the expected values of the independent variables and the variance and covariance parameters obtained from the marginal and joint distributions of these variables. More generally,

$$
E\left[f\left(W_{1}, \ldots, w_{m}\right)\right] \neq\left[f E\left(W_{1}\right), \ldots, E\left(W_{m}\right)\right]
$$

unless $f()$ is strictly linear, which is unlikely. Thus, substituting the expected values of the meteorological variables that influence crop yields 
into Equation (5.1) or any other nonlinear crop yield model will not provide an accurate estimate of the expected yield of a crop. In fact, the use of such an approach implies that the meterological variables that influence crop yield are not random at all, but are fixed at specific values which happen to coincide numerically with $E\left(W_{j}\right)$.

The second reason for introducing climate variability into the analysis involves the traditional aversion of agricultural producers to risk (Halter and Dean 1965; Anderson et a1. 1977; Kunreuther 1972). In general, an individual is said to be risk averse if, given the choice between the expected value of a lottery and an equivalent sum of money with certainty, the individual will always accept the latter. On the other hand, an individual with risk neutral preferences will be indifferent between the two choices (Borch 1968). Given the choice between a number of "risky" activities (i.e., the economic gain from each of these activities is characterized by a probability distribution) a risk-averse individual will consider the tradeoff between the expected return and the variability of the returns before making $h$ is choice, while the risk-neutral individual will always select the activity with the highest expected return.

The relationship between climate variability and risk is as follows. Climate changes that are accompanied by an increase in climate variability can be expected to increase the probabilities associated with the occurrence of extreme meteorological events such as killing frosts, hail storms, and periods of drought. Other things being equal, this will in turn increase the probability in any given year of reduced yields or crop failure, even though the expected value of yields may change very little, or not at all. The fact that the average value of annual yields for a specific crop will, over a long enough period of time, tend toward its new expected value (which might, in itself, be sufficiently high to justify growing the crop in a particular region) may be of little consolation to producers and consumers if the variability of yields about the expected value is quite large. In that context, a few consecutive years of relatively low yields could cause a sharp reduction in the quantity of food produced in those regions where future 
climate changes take the form of increased climate variability. If the geographic scope of these changes should happen to encompass major regions in which specific crops are grown, then decreased food production would also be accompanied by higher prices during periods of low yields and probably by more dramatic fluctuations in crop prices over longer periods of time. Under these kinds of conditions, producers in the affected regions might well attempt to reduce the risks they face by selecting crops which produce less income per acre than traditional crops, but which are also less sensitive to climatic fluctuations. This will have the effect of smoothing out the annual variations in yield due to climate variability. Other things being equal, this will reduce the year-to-year fluctuations in the income derived from these crops, which is tantamount to reducing risk.

Even if climate variability does not change, these are still two good reasons for taking this phenomenon into account. The first involves the detection of $\mathrm{CO}_{2}$-induced environmental changes by producers. For example, if the pre-existing variability in climate yields is very large in a region, it may take many years for producers to determine that a climatically-induced yield change has indeed occurred. What would be involved in this case is a problem in sampling. If the variance of the distribution of crop yields due to existing climate variability was large, producers would have to obtain a larger sample of crop yields in order to determine if there had been a real change in average yields than if the variance in this distribution was much smaller. Since farmers would have to wait a year between sampled observations on crop yields, it might take a very long time to be able to reject the null hypothes is that no change had occurred.

The second reason why it is important to incorporate climate variability in the analysis, even if it remains constant, is that the problems created by the pre-existing variability may be more severe than the problems caused by a potential decrease in yields. As previously mentioned, dramatic year-to-year fluctuations in crop yields are invariably translated into substantial fluctuations in price and/or farm income, depending upon how geographically wide-spread this climatic phenomenon might be. While risk-averse producers 
may be able to dampen these fluctuations by diversifying production, this will be done at the expense of higher expected profits. Thus, even if expected yields did increase somewhat due to favorable $\mathrm{CO}_{2}$-induced environmental changes, it might not make a great deal of difference in the way producers behaved in order to reduce risk.

\subsubsection{The Independence of Environmental Effects on Crop Production}

In Chapter 3.0, the output of a single agricultural commodity was related to the inputs used to produce that good by a production function

$$
Q_{i}=f_{i}\left(x_{i}, z_{i}, \bar{E}\right)
$$

where $Q_{i}$ stood for the total output commodity $i$ by the firm, $x_{i}=\left(x_{i 1}, \ldots\right.$, $\left.x_{i n}\right)$ and $z_{i}=\left(z_{i 1}, \ldots, z_{i k}\right)$ represented vectors of the amounts of variable and allocatable fixed inputs, respectively, used to produce the ith commodity, and $\bar{E}$ was a vector of the values of environmental variables, representing the state of the environment. Thus, the output of the ith commodity depends both upon the values of physical inputs that are under the control of the producer and the state of the environment, including the influence of meteorological variables, which are not under the control of the producer.

The dependence of output on managed inputs $\left(x_{i}, z_{i}\right)$ and unmanaged inputs $(\bar{E})$ to production raises an important question in the economic analysis of the impact of $\mathrm{CO}_{2}$-induced environmental changes on agricultural production. The question is: under what conditions will the impact of changes in the state of the environment on the production of agricultural commodities by the firm be independent of the use of managed inputs? In this instance, the independence of environmental effects on output means that the use of managed inputs by the firm will not be directly influenced by changes in the concentration of $\mathrm{CO}_{2}$ or by changes in meteorological conditions. The concept of independence in this framework does not imply that there are any conditions under which one can entirely ignore the effect of managed inputs on yields. As long as the 
production function includes both types of inputs, there are no conditions under which the influence of managed inputs on yields can be ignored.

It turns out that there is one general condition under which the firm's use of managed inputs will, in fact, be independent of changes in the state of the environment. This will occur when the production function for a commodity can be expressed in additive form as

$$
Q_{i}=f_{i}\left(X_{i}, z_{i}\right)+g_{i}(\bar{E})
$$

so that the partial derivatives of $Q_{j}$ with respect to both $X$ and $Z$ are independent of the state of the environment. Inserting these production functions back into the production problem

$$
\text { Maximize } \begin{aligned}
\Pi= & \sum_{i=1}^{m} P_{i}\left[f_{i}\left(x_{i 1}, \ldots, x_{i n}, z_{i 1}, \ldots, z_{i k}\right)+g_{j}(\bar{E})\right] \\
& -\sum_{j=1}^{n} r_{j} \sum_{i=1}^{m} x_{i j}
\end{aligned}
$$

subject to

$$
\sum_{i=1}^{m} z_{i h}=\bar{z}_{h} \quad \text { for all } h
$$

will then result in factor demand equations which are independent of the state of the environment. That is, in vector format,

$$
x_{i j}=x_{i j}^{*}(P, r, \bar{z}) \quad \text { for all } i
$$




$$
z_{i h}=z_{i h}^{*}(P, r, \bar{z}) \quad \text { for all } i
$$

These factor demand equations for both variable and allocatable fixed inputs can be contrasted with those displayed in Equations (3.8a) and (3.8b) in which no assumption of additivity was made about the individual production functions. The difference between the two sets of factor demand equations is, of course, that those obtained after imposing the restriction of additivity on production do not contain arguments in $E$, the state of the environment. Consequently, changes in the state of the environment will not directly affect the demand by the firm for variable or allocatable fixed inputs when the production functions to which these inputs belong are additively separable in $\bar{E}$ (Berndt and Christensen 1973).

The assumption of additive separability does not entirely eliminate the influence of changes in the state of the environment on the aggregate supply and demand for variable and allocatable fixed factors used in agricultural production. It will be recalled from the example used in Section 3.3 .2 that changes in the state of the environment could affect the equilibrium price and output levels of both variable and fixed inputs supplied to a great many sectors, even if the production related impacts of these environmental changes were confined to the agricultural sector. Two mechanisms through which the effect of $\mathrm{CO}_{2}$-induced environmental changes on agricultural could be transmitted to the input demands of other sectors were identified. First of all, exogenous changes in $\bar{E}$ will have the effect of shifting the aggregate demand curves for inputs supplied jointly by one industry to agricultural and nonagricultural producers. Secondly, $\mathrm{CO}_{2}$-induced changes in the price of agricultural commodities will also affect the aggregate demand for an input. If the industry producing that input jointly supplies other industries with its goods and services, then $\mathrm{CO}_{2}$-induced price changes in the agricultural sector will also cause a shift in the aggregate demand curve for these inputs, leading to a new input price and quantity equilibrium.

In the current case, however, the aggregate factor demand equations consistent with those of the firm expressed in Equation (5.8) will not contain 
an argument in the vector $E$. This is in keeping with the notion of the independence of environmental effects at the level of the individual firm. Consequently, $\mathrm{CO}_{2}$-induced changes in the state of the environment will not have a direct effect on the market prices and output levels of agricultural inputs. On the other hand, changes in the state of the environment can still be expected to influence the marginal cost of producing agricultural commodities both to the individual producers and in agricultural commodity markets. Therefore, changes in $\bar{E}$ will continue to affect the equilibrium prices and output levels of agricultural commodities, quite apart from the assumption of the independence of environmental effects. This means, in turn, that changes in the prices of agricultural commodities will also influence the price-quantity equilibrium in those markets which supply both variable and fixed inputs to the agricultural sector. Thus, the assumption of the independence of environmental effects eliminates the direct influence of changes in $\bar{E}$ on input markets; however, it does not eliminate the effect on input markets of $\mathrm{CO}_{2}$-induced changes in commodity prices.

The independence of environmental effects is an important issue in the applied analysis of the economic impacts of $\mathrm{CO}_{2}$-induced environmental change. Its importance stems from the fact that by using this assumption one can exogenously manipulate crop yields without having to worry about the effect of the environment on input usage, except through the impact of commodity price changes. As such, this assumption not only reduces the complexity of the methods required to model yield response, it also requires little if any modification to agricultural sector and interindustry linkage models used to translate environmental impacts into economic terms. On the other hand, one can easily question the validity of this assumption on empirical grounds. In many situations, such as the interaction between fertilizer and precipitation on well drained soils, there is clearly a strong element of interdependence between managed and unmanaged inputs. The consequences of invoking the assumption of independence, when it is unwarranted, are that it will bias the estimates of the direct economic impact of $\mathrm{CO}_{2}$-induced environmental changes on (a) crop yields, and (b) the market prices and output levels which will prevail in agricultural commodity 
markets. It will also bias estimates of the indirect impacts of these changes on the markets for variable and fixed inputs used in agricultural production.

\subsection{ANALYSIS OF CROP YIELD MODELS}

There are essentially three basic methods outside the realm of economic analys is that can be used to model the response of commercially grown crops to changes in the environment. These include:

- Panel Survey Approach--which is a method that involves the statistical manipulation of expert opinions about future climatological changes and crop response to meteorological conditions in order to arrive at project estimates of crop yields.

- Statistical Yield Models--which employ parametric statistical methods, such as multivariate regression analysis, to estimate the parameters of yield response functions using historical and/or experimental data on crop yields and meteorological variables.

- Simulation Models--which are mathematical representations of the physical, chemical and physiological mechanisms underlying plant growth processes specific to individual crops, and are therefore generalizable to any set of environmental conditions that might be encountered.

The remainder of this chapter will be devoted to a comparative analys is of these three approaches to yield modeling. The methods that can be used to introduce the impact of $\mathrm{CO}_{2}$-induced environmental changes on crop yields directly into agricultural sector models will be discussed in the next chapter. The analysis here will include a general description of the technical features of each method based on specific examples drawn from the relevant literature. In evaluating the comparative strengths and weaknesses of each approach, specific criteria based on the need to quantify the physical impacts of $\mathrm{CO}_{2}$-induced environmental changes on crop yields will be employed. The specific criteria used to evaluate the above methods include: 
- Scope of Environmental Effects--which refers to the ability of these methods to provide estimates of the effect of both first- and second-order impacts of $\mathrm{CO}_{2}$-induced environmental changes on crop yields.

- Geographic Portability--which involves the ability of these methods to predict the impacts of $\mathrm{CO}_{2}$-induced environmental changes on the yield of a crop in a number of different regions without structural modification to the mode $i$ used.

- Meteorological Generality--which relates to the ability of these methods to provide yield estimates consistent with changes in a wide variety of meteorological conditions.

- Incorporation of Technology--which involves the ability of these methods to take into account both the effect of short-term adjustments in managed inputs and the effect of technological change, over longer periods of time, on the yield of a crop.

- Data Requirements and Availability--which refers to the degree of congruity that exists between the input data requirements of a specific method and availability of that data on a systematic basis.

- Statistical Accuracy--which raises the question of how well these methods compare in terms of their ability to accurately forecast crop yields using existing data sets.

\subsubsection{Panel Survey Approach}

Perhaps the most controversial method of estimating the effects of climate change on crop yields is through the use of expert opinions. The development of a yield response model based on the informed judgments of selected members of the scientific community can be faulted on the grounds that it lacks on objective framework, i.e., a mathematical model, for integrating scientific knowledge about environment-plant relationships with the observed behavior of crops under both experimental and field conditions. On the other hand, forecasting yields on a large area basis for many different 
crops is an extremely complex undertaking, subject to a number of various technical problems. The most important of these problems include:

1. existing gaps in the area of statistical yield and simulation model coverage for all major field crops;

2. the tendency of statistical yield models, in particular, to be rooted to the environmental conditions of a specific location, such that these models often do not perform very well when they are used to forecast yields in regions with markedly different environmental cond itions;

3. the fact that errors between observed and predicted yields tend to increase with the difference between "normal" and simulated meteorological conditions;

4. substantial intra-regional differences in observed crop yields which dramatically increases the number of locations for which simulations will have to be conducted for each crop in order to construct accurate estimates of $\mathrm{CO}_{2}$-induced changes in regional and national yields.

In the face of these kinds of problems, the costs of developing and using mathematical models to predict the impact of future increases in the atmospheric concentration of $\mathrm{CO}_{2}$ on crop yields may become quite large. Consequently, it is against the practical backdrop of the problems addressed above, rather than the scientific ideal, that the use of expert judgments to obtain this information ought to be viewed.

\section{Methodo logy}

A possible model for this type of inquiry is contained within. the publication, Crop Yields and Climate Change to the Year 2000, a two volume report, issued by the National Defense University (NDU 1980). The objective of this study was to determine how five global climate scenarios, developed in an earlier phase of the research (NDU 1978), would effect the yield of five crops (winter and spring wheat, corn, rice and soybeans) in seven different countries (the U.S., Canada, Australia, Argentina, the U.S.S.R., India and the 
People's Republic of China). Since each of these crops is not grown cormercially in all of the seven countries, estimates of the effects of hypothetical climate changes on crop yields were performed for only 15 country-crop combinations.

A basic outline of the methodology employed in this study is shown in Figure 5.1. As illustrated in this diagram, the estimation of the impact of the five climate scenarios on crop yields employed three different types of information. The first of these, the base-period BND climate matrix was constructed as follows. First, all of the variable climatological records prior to 1975 were examined for five latitude zones. From these records, three statistics were calculated for each zone: the standard deviation, $S_{T}$, for the distribution of observed departures of mean annual heading--period temperature, $\Delta T$, from the average of the base period (i.e., the period of historical record), the standard deviation, $S_{p}$, for the distribution of observed departures of mean annual crop-year precipitation, $\Delta P$, from the base period average; and the correlation coefficient, $r$, between $\Delta T$ and $\Delta P$. The observed combinations of $\Delta T$ and $\Delta P$ were assumed to come from a bivariate normal distribution (BND), the parameters of which are approximated by the sample distributions. Based on this information a BND climate matrix $(\Delta T, \Delta P)$ was constructed for each region.

The second piece of information represented by the global climate scenarios for the year 2000 was developed in an earlier report (NDU 1978) using probabilistic projections submitted by a panel of climatologists. The five scenarios were characterized as: Large Warming (LG), Moderate Warming (MW), Same as the Last 30 Years (S), Moderate Cooling (MC), and Large Cooling (LC). Each of these scenarios involved specific assumptions about expected changes in $\Delta T$ and $\Delta P$. Each scenario consisted of five latitudinal $B N D$ matrices of $(\Delta \bar{T}, \Delta \bar{P})$ which were constructed by applying the scenario-specific estimates of $\Delta \bar{T}$ and $\Delta \bar{P}$ to the base period BND matrix assuming, first of all, that the distribution of $\Delta T$ and $\Delta P$ in the future would also be characterized by the bivariate normal distribution, and secondly that the ratio of the standard deviation of absolute crop-year precipitation to the average value of absolute crop-year precipitation would be constant. 
BASIC
INPUTS

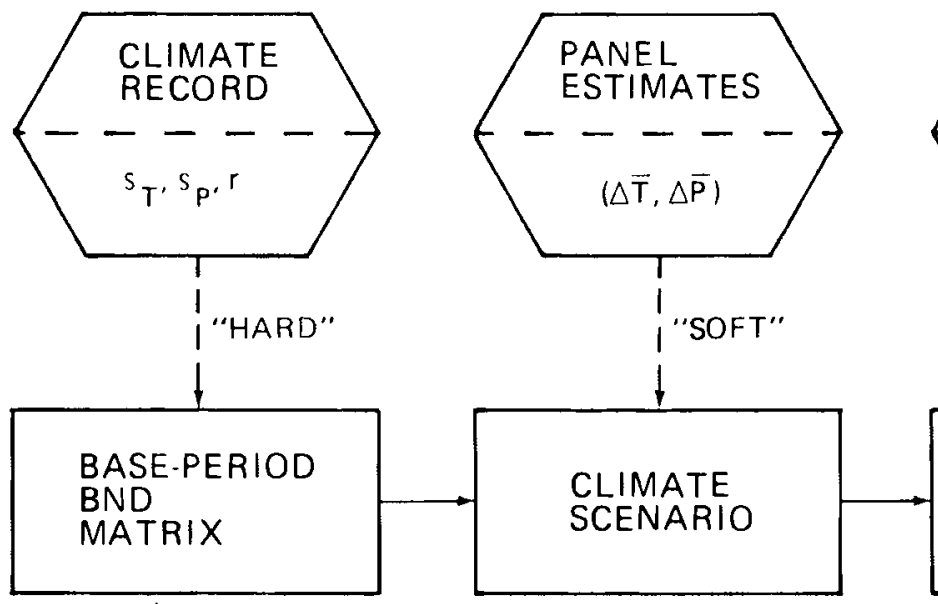

PANEL

ESTIMATES

$Y(\Delta T, \Delta P)$

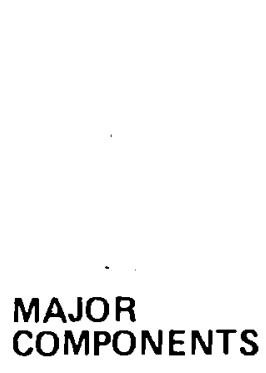

COMPONENTS

BASE-PERIOD
BND
MATRIX

SE LECTIVE SUMMATION OF PROBABILITIES

OUTPUT

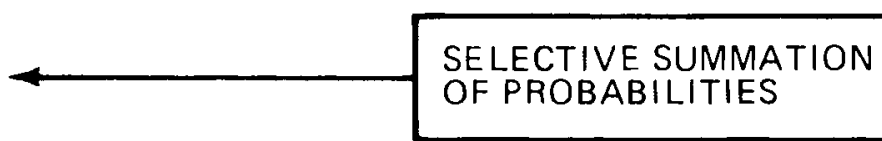

BASE-PERIOD ANNUAL-YIELD MATRIX

FIGURE 5.1. Schematic Diagram of the Climate Response Model [Source: NDU (1980), Figure 1-10] 
The third piece of information, the base period annual yield matrix $Y(\Delta T$, $\Delta P)$ was obtained by the following procedure. Members of an agricultural panel were first asked to fill out a set of grids, one for each of the 15 country-crop combinations, showing their estimates of relative yields (expressed as a percent of average yields during 1972 through 1976) associated with different combinations of $\Delta T, \Delta P$. These estimates were to specifically exclude the effects of government policy and technological changes. The panelists were also asked to rate their own expertise in making these projections. A series of base period annual yield matrices were then calculated by weighting each entry in each grid by a self-assigned expertise rating, adding the weighted values for each panelist and dividing this estimate by the sum of the weights. Subsequently, the distributions of relative annual yields were obtained by superimposing the appropriate zonal BND climate matrices for the base period on the annual yield matrices and suming the BND probabilities that overlay the yield estimates. Finally, using these distributions, the expected value of annual yield was calculated for each of the 15 country-crop combinations. These values were normalized to a base of 100 to facilitate yield comparisons across the different combinations, and this information was used to calculate new yield matrices and base-period yield distributions expressed in terms of normalized relative yields.

Using these three pieces of information, the distribution of annual yields was calculated for each crop in each global yield scenario by shifting the appropriate BND matrix for $(\Delta \bar{T}, \Delta \bar{P})$ until it was centered on the yield entry in the base period annual yield matrix for which $\Delta T=\Delta \bar{T}$ and $\Delta P=\Delta \bar{P}$ and then suming the BND probabilities which overlay these entries. The resulting distributions of normalized relative crop yields were then analyzed to determine how each of the scenarios affected both the expected value and the variability of yields.

The results of this analysis indicated, among other things, that climate changes had the greatest impact on crops grown in the northern higher middle latitudes, which included both spring and winter wheat. The effect of the large and moderate warming scenarios on Canadian and U.S. spring wheat was 
favorable. On the other hand, large and moderate warming tended to produce very slight decreases in the yields of crops grown in the northern lower middle latitudes of the U.S., namely: soybeans, corn, and winter wheat. Although the study did not conclude as much, this evidence might also imply some geographic movement in the northern range of soybeans, corn and wheat should either of these two scenarios be realized in the future.

\section{Evaluation}

The chief advantage of the approach described above is that, despite its simplicity, it has the capacity to generate quantitative answers about the effects of a variety of different types of climate changes on crop yields. By projecting distributions rather than point estimates of crop yields this method is able to capture the effect of climate changes on both the expected value and the year-to-year variability of yields. In the NDU study simplifying assumptions about the joint distribution of $\Delta \bar{T}$ and $\Delta \bar{P}$ in the future were made that may not be justified. However, it is important to point out that other assumptions could have been made and the analys is could have been extended further to examine the sensitivity of crop yields to changes in the types of distributions used to characterize future occurrences of $\Delta \bar{T}, \Delta \bar{P}$. Thus, the method is also extremely flexible in terms of its general ability to portray the stochastic influences of meteorological conditions on crop yields.

The application of the specific criteria identified earlier in Section 5.2 to the approach embodied in the NDU study can be summarized as follows:

Scope of Environmental Effects. The NDU study focused exclusively on the effects of climate change on crop yields. It is conceivable that the effect of $\mathrm{CO}_{2}$ on crop yields due to enhanced photosynthetic activity as well as second-order effects could be incorporated into the method by asking panelists to identify the influence of each type of environmental effect on crop yields, holding other effects constant. This was how the influence of technology was separated from the influence of climate on crop yields in the NDU study. The problem with this approach, in general, is that it neglects the possible 
interaction between the different effects by assuming that the combined influence of all effects on crop yields is strictly additive.

Geographic Portability. The ability to extend this approach to a different set of regions is limited only by the availability of base-period data on the values of the relevant meteorological variables.

Meteorological Generality. In the NDU study only two meteorological variables were employed. The number of variables could be increased; however, doing so could pose problems in obtaining the parameters of the joint distribution used to characterize climate if the historical records for individual regions contain too few observations. More importantly, increasing the number of meteorological variables would substantially increase the number of yield entries that would have to be supplied by panelists for each country-crop combination.

Incorporation of Technology. Panelists in the NDU study were also asked to provide probabilistic responses regarding the effect of future technological change on crop yields in the absence of climate change. While the effect of technological change on crop yield was not strictly comparable with the impacts of the climate changes (because the latter were expressed in terms of normalized relative yields), the results of the survey tend to suggest that technology-induced increases in crop yields will, in general, more than compensate for selective yield reductions due to climate change. of course, the actual changes in technology that occur in the future will partly reflect adjustments that are made to foreseeable climate changes.

Data Requirements and Availability. This will be dictated entirely by the selection of meteorological variables and regions to be used in any future study. The period of meteorological observations used in the NDU study ranged from 10 to 60 years. In general, the shorter the period of record that is used the greater the probability that the parameters of the sampled distribution of meteorological events will differ from the "time" parameters of the actual distribution.

Statistical Accuracy. The distributions of normalized crop yields constructed from the panel responses could not be validated directly against 
historical yields because the yield estimates supplied by the panelists were frozen in current technology. This was remedied in the study by constructing two equivalence factors to embody the effect of technological change. When these were applied to the normalized yield estimates, departures of the adjusted average yields from the trend value based on observed yields were never greater than $6 \%$.

In general, then, the approach embodied in the NDU study does not fare as badly as might be expected, given the fact that it is based on the use of much so-called "soft" data. The weakest element of the approach is that the number of meteorological variables must be kept fairly small to avoid conceptual difficulties in providing yield estimates for a large number of climate states. On the other hand, the current paucity of information regarding the effects of $\mathrm{CO}_{2}$ on future climates may dovetail nicely with this approach.

\subsubsection{Statistical Yield Models}

The term statistical yield models is used in this report to embrace a fairly broad range of models which employ multivariate regression procedures to determine the influence of a vector of independent variables on the yield of a crop, using time series and/or cross sectional observations on yields and the independent variables. The standard linear model associated with this approach is

$$
Y=\beta_{0}+\sum_{i=1}^{m} \beta_{i} W_{i}+\varepsilon
$$

where $Y$ denotes the yield of a crop, $W_{i}$ represents a vector of meteorological terms which may include transformations of meteorological variables as well as interactions between them, and $\varepsilon$ is a normally distributed error term with a mean of zero and variance equal to $\sigma^{2}$. Typically, the model is "fitted" to a set of observations on $\left(Y, W_{1}, \ldots\right.$, $W_{m}$ ) by means of ordinary least squares (OLS) in order to obtain estimates of the mean values and standard deviations of the parameters $\beta_{0}$ and $\beta_{1}, \ldots$, $\beta_{m}$, as well as an estimate of the standard error (standard deviation) of the 
difference between the observed and fitted values of $Y$ and the coefficient of determination, $r^{2}$, which estimates the fraction of the observed variance in $Y$ which is explained by the variation in the independent variables.

Many of the problems that are encountered in estimating the parameters of statistical crop yield models and then using these models to forecast yields are directly related to the use of regression analysis. The first problem, which is more in the line of clarification, has to do with the conceptual meaning attached to the parameter estimates in these models. Hundtoft et al. (1971) has made the distinction between models that describe the outcome of physiological processes using true functional relationships and those which characterize these processes with empirically-derived functions. In the former type of model, specific coefficients represent constants with a clear physical meaning, while in the latter case these coefficients are empirical parameters that have no physical significance.

Statistical crop yield models are representative of the latter type of approach. Although one is always free to omit a variable from a regression model if the parameter estimate associated with it either is statistically insignificant or does not conform to a priori expectations regarding its sign or magnitude, the parameters of these variables are, in general, statistical artifacts of the OLS estimation procedure. On a conceptual level, each of the $B_{j}$ in a crop yield model can be regarded as a "treatment effect," e.g., the influence of a unit increase in $w_{j}$ upon yield. Apart from this, they have no specific meaning related to the physiology of plant growth.

In the absence of a systematic theoretical framework for including explanatory variables into these models, stepwise regression procedures are typically employed to select a subset of variables from a much larger list of possible candidates. Given that the major objective of crop yield models lies in the area of prediction, this somewhat arbitrary method of model building cannot really be faulted. On the other hand, it is usually true that a number of variables which the researcher knows will influence the yield of a crop must be omitted, either because there are no observations on these variables, or else because there are too few observations in the data set to include 
additional variables. If the omitted variables represent truly random influences on crop yields, then their effect on yield is captured correctly by the error term. The omission of variables whose variation is systematically related to the variation in yields is a different matter. Typically, the omission of these types of variables is rationalized by the "ceteris paribus" condition, which simply means that variables not included in the model did not vary over the range of observations used to estimate the model parameters. If this is not the case, again an empirical rather than the theoretical issue, then the model will be improperly specified and the estimates of the parameters will be biased. More importantly, the model will not be as good a forecasting instrument as it would be if these variables were included.

In order to avoid these problems, statistical crop yield models in most cases are specific to a particular location or region where climate conditions, cropping practices, soil characteristics and technology are fairly homogeneous. This practice represents an attempt to satisfy the ceteris paribus condition, primarily to improve the quality of the forecasts that will be produced by the model. If the region, or regions, from which the data are drawn are in fact characterized by homogeneity with respect to cultural practices and environmental variables not included in the model, one can expect that the parameter estimates contained within the model will have practical significance both for assessing crop conditions during the growing season and for making final yield predictions in that region. On the other hand, the value of the model in performing these functions well is often purchased at the necessary price of a loss in generality as $f$ ar as making good predictions of yields outside the region from which the data are drawn.

An equally serious problem with many statistical yield models. is their inability to provide accurate predictions of crop yields during years characterized by abnormally sharp departures from normal weather patterns. In statistical terms, extreme events can be characterized as observations which are outliers with respect to the regression surface that is fitted by the OLS procedure. If the number of outliers is small, such as one might expect in the case of extreme events, then the effect of these outliers on the "fit" of the model may be very small. 
However, a problem in estimation arises when the variation in the independent variables contained in the data set used to estimate the model is not representative of the "true" variation in these variables in the population from which they are drawn. This can occur when the period of historical record is fairly short-- in meteorological terms, less than 50 years. In this type of situation it is possible that what appears to be an outlier in the data is not an outlier in the population. Unfortunately, there is no statistical remedy for this problem since regression analysis depends on the assumption that the sampled data are representative of the population. As a purely practical problem, however, using too short a historical record may lead one astray as $f a r$ as the true values of the regression parameters are concerned, and the systematic effect of extreme events on yields may be missed.

Nevertheless, even if this problem does not exist (and there is no way of detecting it) the fact remains that the predictive ability of regression models, in general, decreases as the independent variables in the sample take on extreme (high and low) values. This may not be a serious problem as far as forecasting is concerned if the values of the independent variables used in making the forecast lie within, say, a standard deviation of the mean values of the variables in the data set used to estimate the parameters of the model. On the other hand, if the forecast changes in the explanatory variables lie well outside this range, as may be the case with $\mathrm{CO}_{2}$-induced climate changes, then the variance about the predicted value of crop yield will be unacceptably large.

A final general problem with statistical crop yield models relates to model validation. The acid test of a regression model that is used to make predictions is that it does a good job of forecasting. Given a number of different possible models to explain the same phenomenon, the procedure generally used to select the best candidate is to obtain an independent data set (i.e., a different one than was used to estimate the model), and use the values of the explanatory variables in this data set to predict the values of the dependent variables and then compare the root mean square error (RMSE), which is the standard deviation of the difference between the observed and 
predicted values of the dependent variable, for each model. When only a single model is involved, this procedure is referred to as model validation. Generally speaking, the RMSE of a good model will not differ substantially from the standard error of the regression model when corrected for differences between the mean value of the dependent variable in the two data sets.

Unfortunately, validating statistical crop yield models is not as easy as it sounds. The reason for this is that the lack of generality of these models, combined with the need to employ a large number of observations from the historical record to perform the regression, often makes it very difficult to find an independent data set. Thus, reseachers who develop these models are frequently forced to express the forecasting abilities of their models in terms of how well the model fits the data used to estimate its parameters. This is a poor test of the flexibility of the model as a forecasting instrument. It also complicates the problem of determining how well these models will perform under environmental conditions that may differ from those contained in the original data set.

Since statistical yield models differ in many other respects, the following review of selected models is presented in order to provide a broader overview of methodological features and problems not directly related to the statistical method employed to estimate their parameters.

\section{Methodo logy}

The most basic approach to yield modeling is perhaps best exemplified by Thompson's (1969) wheat model. The form of the model is

$$
y=\beta_{0}+\sum_{i=1}^{m} \beta_{i} w_{i}+\sum_{i=1}^{m} \alpha_{i} w_{i}^{2}+\phi_{1} t_{1}+\phi_{2} t_{2}+\phi_{3} t_{2}^{2}+\varepsilon
$$

where $m=12$ is the number of meteorological variables in the model. Each of the meteorological variables selected by Thompson was expressed in terms of departures from "normal" monthly temperatures and precipitation. This was done to eliminate the problem of multi-colinearity between the explanatory 
variables. Specific meteorological variables included in the model were preseason precipitation from August through March and the transformed (squared) values for rainfall and mean temperatures in the months of April through July. Three technology terms were used in the model:

1. a linear time trend variable, $t_{1}$, which increases by one each year from the beginning of the record (1920) through 1945, after which it becomes a constant,

2. a linear term, $t_{2}$, with a value of zero prior to 1945 and a value of one in 1946 that increases by one in each successive year until 1968 ,

3. the square of the second term which was introduced to capture changes in the rate of technological improvement after World War II.

The mode 1 was estimated for six different states in the Great Plains, using meteorological and yield data for the period 1920 through 1968 . The regression coefficients and the standard error of the residuals for each state are shown in Table 5.1. The standard errors shown at the bottom of this table are fairly small in relation to the variance of the distribution of annual yield for each state, as determined from the historical record. The ratios of the standard error of the residuais for each state to historical average yields are in the range of 0.1 to 0.18 , a bit high for forecasting purposes. Cross sectional differences in the magnitude and signs of the parameters estimates shown in Table 5.1 suggest considerable technological and environmental heterogeneity on a state-to-state basis. Thus, it is unlikely that pooling the data from the different states and re-estimating the model using regional dumy variables to account for these differences would substantially improve the standard errors and the resulting quality of the forecasts.

There have been essentially three different types of modifications made to the Thompson approach. The first of these consists of the introduction of derived meteorological variables into the regression equations. The most widely used types of derived variables that have been developed to improve statistical crop yield models include (a) the use of heat and soil moisture 
TABLE 5.1. Regression Coefficients for Multiple Regression Analys is of Wheat Yields

\begin{tabular}{|c|c|c|c|c|c|c|c|}
\hline Independent $\mathrm{Var}$ & iable & $\begin{array}{l}\text { North } \\
\text { Dakota }\end{array}$ & $\begin{array}{l}\text { South } \\
\text { Dakota }\end{array}$ & Kansas & Oklahoma & Illinois & Indiana \\
\hline $\begin{array}{l}\text { Trend 1920-1945 } \\
\text { Trend } 1945-1968\end{array}$ & $\begin{array}{l}\text { Linear } \\
\text { Linear } \\
\text { Quadratic }\end{array}$ & $\begin{array}{c}10.113 \\
0.045 \\
0.572 \\
---\end{array}$ & $\begin{array}{c}11.789 \\
-0.052 \\
0.442 \\
---\end{array}$ & $\begin{array}{c}12.215 \\
0.066 \\
0.498 \\
---\end{array}$ & $\begin{array}{r}13.198 \\
0.021 \\
0.981 \\
0.021\end{array}$ & $\begin{array}{r}15.893 \\
0.158 \\
0.902 \\
0.005\end{array}$ & $\begin{array}{r}13.488 \\
0.217 \\
0.933 \\
0.008\end{array}$ \\
\hline $\begin{array}{l}\text { August-March } \\
\text { Prec ipitation }\end{array}$ & $\begin{array}{l}\text { Linear } \\
\text { Quadratic }\end{array}$ & $\begin{array}{r}0.926 \\
-0.021\end{array}$ & $\begin{array}{r}0.416 \\
-0.179\end{array}$ & $\begin{array}{l}0.631 \\
-\ldots\end{array}$ & $\begin{array}{l}0.380 \\
-0.027\end{array}$ & $\begin{array}{l}-0.221 \\
-0.001\end{array}$ & $\begin{array}{l}-0.229 \\
---\end{array}$ \\
\hline April Precipitation & $\begin{array}{l}\text { Linear } \\
\text { Quadrat ic }\end{array}$ & $\begin{array}{l}0.426 \\
0.018\end{array}$ & $\begin{array}{r}1.568 \\
-0.614\end{array}$ & $\begin{array}{r}0.223 \\
-0.048\end{array}$ & $\begin{array}{r}0.186 \\
-0.048\end{array}$ & $\begin{array}{r}0.161 \\
-0.249\end{array}$ & $\begin{array}{l}-0.141 \\
---\end{array}$ \\
\hline April Temperature & $\begin{array}{l}\text { Linear } \\
\text { Quadratic }\end{array}$ & $\begin{array}{r}0.096 \\
-0.028\end{array}$ & $\begin{array}{r}0.225 \\
-0.045\end{array}$ & $\begin{array}{l}-0.095 \\
---\end{array}$ & $\begin{array}{l}-0.321 \\
---\end{array}$ & $\begin{array}{l}-0.057 \\
-0.013\end{array}$ & $\begin{array}{r}0.015 \\
-0.022\end{array}$ \\
\hline May Precipitation & $\begin{array}{l}\text { Linear } \\
\text { Quadratic }\end{array}$ & $\begin{array}{r}0.900 \\
-0.061\end{array}$ & $\begin{array}{r}-0.158 \\
0.132\end{array}$ & $\begin{array}{r}0.191 \\
-0.285\end{array}$ & $\begin{array}{l}-0.618 \\
-0.120\end{array}$ & $\begin{array}{l}-0.549 \\
-0.077\end{array}$ & $\begin{array}{l}-0.317 \\
-0.072\end{array}$ \\
\hline June Precipitation & $\begin{array}{l}\text { Linear } \\
\text { Quadratic }\end{array}$ & $\begin{array}{l}0.701 \\
---\end{array}$ & $\begin{array}{l}0.433 \\
-.-\end{array}$ & $\begin{array}{l}-0.327 \\
---\end{array}$ & $\begin{array}{r}0.111 \\
-0.235\end{array}$ & $\begin{array}{l}-1.339 \\
-0.228\end{array}$ & -0.730 \\
\hline June Temperature & $\begin{array}{l}\text { Linear } \\
\text { Quadratic }\end{array}$ & $\begin{array}{l}-0.417 \\
--\end{array}$ & $\begin{array}{l}-0.303 \\
---\end{array}$ & $\begin{array}{l}-0.101 \\
-0.005\end{array}$ & $\begin{array}{r}0.392 \\
-0.163\end{array}$ & $\begin{array}{l}-0.351 \\
--\end{array}$ & $\begin{array}{l}-0.121 \\
---\end{array}$ \\
\hline July Precipitation & $\begin{array}{l}\text { Linear } \\
\text { Quadratic }\end{array}$ & $\begin{array}{l}0.381 \\
---\end{array}$ & $\begin{array}{r}-0.961 \\
0.008\end{array}$ & --- & --- & --- & --- \\
\hline July Temperature & $\begin{array}{l}\text { Linear } \\
\text { Quadratic }\end{array}$ & -0.214 & $\begin{array}{l}-0.431 \\
--\end{array}$ & --- & --- & -- & -- \\
\hline Standard Error in bu & & 2.9 & 2.4 & 2.5 & 2.8 & 2.8 & 3.1 \\
\hline
\end{tabular}

Source: Thompson 1969. 
"stress days" (McQuigg 1975; Anderson et al. 1977; Shaw 1974), which represent the number of days during a particular month when the temperature (or soil moisture content) is above (or below) a specific value, and (b) growing degree day units (Cross and Zuber 1972; Ramirez and Bauer 1973; Nield and Seely 1977) which account for the accumulated temperature deviations from a threshold value. A second improvement involves the addition of terms to statistical yield models to account for the effect of managed inputs on yield, particularly the application of fertilizer (Nelson and Dale 1978a, 1978b; Feyerherm 1979; Feyerherm and Paulsen 1981) and cultural practices (Runge and Benci 1975; Feyerherm 1979; Feyerherm and Paulsen 1981). The study by Nelson and Dale (1978a) showed that corn yield models which used the annual average input of nitrogen provided better predictions of corn yields in Indiana than did models using time trends. This is consistent with post-war technological improvements in corn yields which were due in large part to more fertilizerintensive cultivation practices in the corn belt.

Perhaps the most serious problem with the approach used by Thompson and many of his contemporaries is that it contained no explicit link between the meteorological variables that were dated on a calendar bas is and the phenology of crop development. For example, if potential spring rains delayed the planting date of a crop in a specific year, the effect of meteorological influences on the subsequent development of the crop would not be in accordance with the parameter estimates of the model. This would be true, first, because the "normal" relationship between calendar time and crop phenology will have been altered due to abnormally high spring rains and, secondly, because the relationship between meteorological conditions and the growth of the crop generally differs depending upon the stage of plant development. This explains why the forecasts obtained from crop yield models are generally less reliable in situations where the meteorological conditions in the forecast period depart sharply from normal trends.

Correcting this type of problem requires that meteorological variables be dated with respect to particular phenological stages. However, in order to implement such an approach it is also necessary to relate the occurrence of different stages of crop development to the meteorological events on which the 
rate of plant maturity depends. This is accomplished by constructing a crop calendar which is specific to each of the annual sets of observations contained in the data used to estimate the parameters of the model. In some cases, the dates of occurrences for specific phenological events can be obtained from USDA and state data sources, although the quality of phenological data is uneven. In the absence of this data, the timing of spec if ic developmental stages must be developed from biometeorological time scale (BMTS) models (Robertson 1968).

A relatively simple corn yield model involving the use of meteorological variables that are dated according to phenological events in conjunction with a biometeorological time scale model is contained in several studies by Nield and Seely (1976), Nield and Richman (1979) and Nield et al. (1979). The model used in these studies is

$$
Y=\beta_{0}+\sum_{i=1}^{3} \beta_{i} W_{i}+\beta_{4} W_{3}^{2}+\phi_{1} t+\varepsilon
$$

where the meteorologic variables are

$$
\begin{aligned}
& W_{1}=\text { Preseason precipitation (September } 1 \text { to planting) } \\
& W_{2}=\text { Temperature stress days (days over } 95^{\circ} \mathrm{F} \text { between ear formation } \\
& \quad \text { and denting kernals) } \\
& W_{3}=\text { Precipitation from tasseling to kernals in dough. }
\end{aligned}
$$

The time scale model was estimated as follows. Fourteen different phenological stages for corn were identified and each assigned a value between 0.5 and 10.0 in the order of their occurrence according to the method devised by Hanway (1971). These values were used as the dependent variable in the regression analysis. The accumulated growing degree days (GDD) from planting to the occurrence of each stage were used as the independent variable in the regression analysis. The model was estimated using phenological observations and growing degree days taken at weekly intervals from an extreme range of planting dates for three different hybrids at Mead, Nebraska in 1975. The results of the regression analysis are shown in Table 5.2 . 
TABLE 5.2. Growing Degree Days Predictions

\begin{tabular}{rllllll}
$\begin{array}{c}\text { Maturity } \\
\text { Class (days) }\end{array}$ & & Prediction Equation & & $n$ & $\frac{r^{2}}{}$ & S.E. \\
\cline { 1 - 2 } 95 to 100 & Stage $=-0.40+0.0042$ GDD & & 128 & 0.98 & 0.40 \\
111 to 120 & Stage $=-0.44+0.0038$ GDD & & 133 & 0.98 & 0.44 \\
121 to 130 & Stage $=-0.47+0.0037$ GDD & & 135 & 0.97 & 0.47
\end{tabular}

Source: Nield and Seely, 1977.

The approach described above is currently being implemented on a real-time basis on the Agnet reporting system (Thompson 1981) in the state of Nebraska to predict crop yields on a weekly bas is during the growing season. These models have also been used to simulate the impact of hypothetical climate scenarios on corn production throughout the state. The simulations are conducted by first using the parameters of the time scale model to determine when specific stages of the crop occur consistent with the simulated pattern of accumulated growing degree days. Once the dates of the important stages of crop development have been determined, the corresponding values of the relevant meteorological variables $\left(W_{2}\right.$ and $W_{3}$ ) are read from the simulated input data. The values of these meteorological variables are then fed into the regression equation, along with the simulated planting date and the value of $t$, to provide a yield forecast that is consistent with simulated meteorological conditions.

While the approach developed by Nield and Seely is indicative of current efforts to overcome the problems associated with predicting yields under highly variable meteorological conditions, other features of the model are not substantially different from the traditional Thompson-type model. Efforts to overcome some of the other shortcomings of the traditional approach have led to the development of models by Baier (1973), Robertson (1974), Haun (1974), Feyerherm (1979) and Feyerherm and Paulsen (1981) which appear to be applicable over a wide range of environmental conditions, technology and cultural practices. Particularly important in this regard are the spring and winter wheat models of Feyerherm and Paulsen (1981) which explicitly incorporate the 
effects of abrupt year-to-year yield changes due to weather and pests, variations in soil type, and long-term shifts due to technological

improvements through added nutrients, the development of new cultivars, weed and pest control and other cultural practices.

The structure of both models is fairly complex and will not be presented in detail. Since the two models are fairly similar, the discussion that follows will focus on the winter wheat model only. The first step of the analysis involved the development of a model to explain the variation in grain yields obtained from a large number of experimental plots as a function of three yield components: (1) weather (WX), nitrogen (NI), and soils (SO). Thus, standardized plots yields for each of three cropping practices can be expressed as

$$
\text { STDYLD }_{i}=K+W X_{i}+N I_{i}+S_{i}
$$

where $i=1,2,3$ denotes cropping practice, $k$ is the constant in the regression function for $\operatorname{STDYLD}_{i}$, and where the weather component $W X_{i}$, represents the sum of soil moisture (MOIST ${ }_{j}$ ) and temperature (TEMPRE) subcomponents.

Separate yield component regression equations were estimated using yield, nitrogen input and soils data from the experimental plots and daily weather data showing precipitation, and minimum and maximum temperatures of weather stations near the experimental sites. In a 11, the data set contained 726 observations for winter wheat covering the range of dryland conditions found in the U.S. Great Plains and cornbelt. The yield component regression equations associated with Equation (5.12) can be expressed in terms of the ir arguments as follows

$$
\begin{aligned}
& \text { MOIST }_{i}=f_{1 i}\left(\text { PR }_{i}, \text { ANP, ET } i\right) \\
& \text { TEMPRE }=f_{2}(T X, P R, T 10, T 25, X P R)
\end{aligned}
$$




$$
\begin{aligned}
& N I_{i}=f_{3 i}\left(\text { NITR }_{i}\right) \\
& S O_{i}=f_{4 i} \text { (OM, STEX PEDORN) }
\end{aligned}
$$

where

$$
\begin{aligned}
& \text { PR = accumulated precipitation (stage specific) } \\
& \text { ANP = long-term average annual precipitation } \\
& \text { ET = accumulated evapotranspiration (stage specific) } \\
& \text { TX = mean daily maximum temperature (stage specific) } \\
& \text { T10 = mean number of degree days for } T>10^{\circ} \mathrm{C} \text { (stage specific) } \\
& \text { T25 = mean number of degree days for } T>25^{\circ} \mathrm{C} \text { (stage specific) } \\
& \text { XPR = accumulated precipitation in excess of } 10.16 \mathrm{~cm} \text { (stage specific) } \\
& \text { NITR = elemental nitrogen applied } \\
& \text { OM = (1,0): } 1 \text { if soil organic matter }>20 \% \text {, } 0 \text { otherwise } \\
& \text { STEX = (1,0): } 1 \text { for sandy loam or loamy sand soil, } 0 \text { otherwise } \\
& \text { PEDORN = (1, 0): } 1 \text { for poorly drained soil, } 0 \text { otherwise } \\
& \text { Weather-related variables in Equation (5.12a) and (5.12b) were calculated } \\
& \text { between Specific phenological stages. Crop calendars for each location were } \\
& \text { determined from climatic parameters by a method devised specifically for the } \\
& \text { model. This method can be implemented fairly easily for regions where } \\
& \text { phenological observations are not a part of the historical record. The values } \\
& \text { for ET were calculated using soil moisture budgets developed by Baier et al. } \\
& \text { (1965, 1966, 1971, 1972). Simulation of the model in regions where these } \\
& \text { budgets are invalid would require the development of region-specific } \\
& \text { calculations for ET. However, these methods are well developed and widely } \\
& \text { applicable. } \\
& \text { In the second step of the analysis, the standardized yield estimates } \\
& \text { obtained from the previous step were modified to take into account the effects } \\
& \text { of cropping practices, the occurrence of episodal events such as pest } \\
& \text { outbreaks, and genetic factors specific to different cultivars. The resulting } \\
& \text { plot yield values (PLOTYL) were computed as }
\end{aligned}
$$




$$
\text { PLOTYLD }=(1-E E F)(C Y A)\left[\sum_{i=1}^{3} C P_{i}\left(\text { STDYLD }_{i}\right)\right]
$$

where EEF and CYA are the regional values of an episodic events factor and an average yielding ability index, respectively, and $\mathrm{CP}_{\mathfrak{j}}$ is the proportion of wheat 1 and under cropping practice $i$. Next, the calculated values for PLOTYLD obtained from Equation (5.13) and a time trend were used as independent variables in a regression equation designed to explain the variation in regional yields (REGYLD). The equation estimated was

$$
\text { REGYLD }=\beta_{0}+\beta_{1}(\text { PLOTYLD })^{2}+\beta_{2}(\text { YR-55) }
$$

where $Y R-55$ is a time trend constructed such that harvest year is equal to $Y R$ and $Y R-55=13$ if $Y R>68$.

Finally, a local adjustment factor (ADJ) was introduced for each weather station and region (crop reporting district). This factor was used to adjust for the bias in REGYLD due to: (1) distance-related variations in weather between the location of the weather centers and the experimental plots (Dugas et al. 1981); (2) input values for soil characteristics which deviate from the "true" regional value; and (3) nonmodeled sources of areal variation in yields. The adjustment factor used was

$$
A D J=\overline{U S D A}-\overline{R E G Y L D}
$$

where $\overline{U S D A}$ is the average value of the USDA yield estimate for each crop reporting district and $\overline{R E G Y L D}$ represents the average of REGYLD estimates calculated over a specific number of years. Once this adjustment was made, the final model value of yield

$$
\text { MODYLD = REGYLD + ADJ }
$$




$$
\text { MODYLD }=\text { REGYLD + ADJ }
$$

was calculated. This represents the predicted value of regional yield and is the final output of the model.

The model was validated using data from 37 crop reporting districts in Montana, South Dakota, Nebraska, Kansas, Oklahoma, Texas and Colorado for the years 1955 through 1956. The average estimated yield for this data set according to USDA data was $16.1 \mathrm{bu} / \mathrm{a}$. The RMSE of the model (the standard deviation of the difference between the USDA and model estimate) was 1.2 , or 7.5\% of the USDA average yield figure.

The performance of an earlier version of this model was compared with that of models produced by the Center for Climatic and Environmental Assessment (CCEA) by Stuff et al. (1979). In contrast to the model developed by Feyerherm and Paulsen, the CCEA models are region-specific, while the approach shown above can be applied with only one adjustment to a great number of regions. Nevertheless, in a 10 year test (1967 to 1976) for the Great Plains the RMSE for the CCEA models was 1.5 compared with 1.3 using the current approach. This suggests that increasing the generality of statistical crop yield models may be accomplished without any loss in predictive ability. However, the evidence of this assertion is still fragmentary in terms of the number of models for which the claim can be made.

\section{Evaluation}

The basic advantages of statistical crop yield models are, first, that they can be constructed from readily available data sources with a relatively limited amount of effort (compared, for example, to simulation models) and, secondly, the expected value and the variance of crop yield can usually be calculated without actually having to simulate the many joint occurrences of meteorological events. Unfortunately, the region-crop coverage of these models is somewhat spotty, limited in the U.S. primarily to spring and winter wheat, corn and soybeans in the Great Plains and the cornbelt states. If th is approach is to be utilized on a systematic basis to simulate the effects of $\mathrm{CO}_{2}$-induced environmental changes on crop yields, this coverage will have to be extended to additional crops of major importance. 
The application of the specific criteria identified earlier in Section 5.2 to statistical crop yield models can be sumarized in the following terms:

Scope of Environmental Effects. Statistical crop yield models generally consider only the impact of meteorological conditions on crop yields. However, more general models are being developed to take into account the influence of different soil characteristics and pest-related episodal events on yields. The direct effect of $\mathrm{CO}_{2}$ on crop yield cannot be modeled because the observed variation in the atmospheric concentration of $\mathrm{CO}_{2}$ over the last 50 or so years is probably too small to estimate the regression parameter which will capture that effect.

Geographic Portability. This is perhaps the weakest characteristic of statistical crop yield models. In most cases, these models are region-specific and efforts to use them to forecast yields in different regions generally result in large forecast errors. Models with a substantially greater geographic range are being developed for a few crops, but they are in the minority.

Meteorological Generality. From a statistical standpoint, regression models do not in general provide reliable forecasts under meteorological extremes. The range of the extremes that can be modeled, without substantially increasing the variance of the parameter estimates, can be increased by dating inputs according to crop developmental stages and linking the dated values of meteorological variables to specific phenological events. This procedure will reduce the problem, but never eliminate it statistically.

Incorporation of Technology. Methods have been devised to include the effects of both long-term technological changes as well as the influence of variations in a small range of managed inputs (nitrogen; irrigation water and cropping practice) on crop yields. Hence, the independence of environmental effects need not necessarily be assumed when using models that contain these features.

Data Requirements and Availability. Because the parameters of these models must be estimated by empirically-based procedures data availability is 
ordinarily not an unsurmountable problem. On the other hand, the availability of phenological data is usually limited and the need to resort to meteorological time-scale models undoubtedly results in errors linking calendar dates to phenological stages.

Statistical Accuracy--Accuracy is a relative question. The RMSE of a good crop yield model will generally be less than $10 \%$ of the average values of yield in the data base used to validate the model. However, forecast errors obtained by using existing data sets may not reflect the accuracy of model predictions under simulated meteorological conditions if the variabilty in the simulated climate is substantially different than is represented in the validation data base.

\subsubsection{Simulation Models}

Statistical crop yield models represent a reductionist approach to the modeling of crop growth processes. The complexity of the mechanisms that underlie plant growth is generally ignored in favor of an approach that isolates a single measure of physical output and then tries to relate this measure to a vector of environmental variables, the selection of which is accomplished on a trial and error basis using multivariate regression analysis. In contrast, crop growth simulation models take a more wholistic approach to the problem. In general, these models can be viewed as an attempt to integrate the constituent processes of plant growth such as photosynthesis, respiration, transpiration and morphogenesis into a system whose component parts can be described mathematically.

Crop simulation models are based on the assumption that the state of the system at any given point in time can be described by the values of the state variables. Examples of state variables include the weight and number of specific plant organs and the supplies of plant nutrients and carbohydrates that are available for growth. In dynamic models, the values of these state variables are updated at each iteration of the model by rate variables which determine the incremental changes in the state variables. The values of the rate variables in the system are, in turn, determined by auxiliary variables which are state-dependent and forcing variables which represent the effect of 
exogenous influences on the system. Feedback effects with in and between component processes which influence the values of rate variables are taken care of by auxiliary variables, while the impact of natural and anthropogenically-induced changes in the environment on rate variables is determined by forcing variables. Thus, at any given point in time, changes in the state of the system will depend not only on exogenous factors, but also on the state of the system as a whole.

The concepts that underlie the systems approwen to crop simulation modeling can be more clearly illustrated by a system diagram of the cotton simulation model, GOSSYM, developed by Baker et a1. (1979). : igure 5.2 represents a schematic diagram which focuses on the carbohydrate $\left(\mathrm{CH}_{2} \mathrm{O}\right)$ and nitrogen $(N)$ balance that is maintained in the model. Here standard systems dynamics notation is used; rectangles represent pools of material (state variables) of definite size, while pools of indefinite size (also state variables) are represented by the irregularly-shaped enclosures; the valve shaped characters represent regulators of the rates of flow between the pools (rate variables); solid lines represent material flows; and dashed lines to the flow values represent information.

Concentrating for the moment on that part of the system which relates to leaf development, it can be seen that the model contains pools of nitrogen and labile carbohydrates which enter the system through the processes of photosynthesis and transpiration. The rate at which $\mathrm{CO}_{2}$ is reduced to $\mathrm{CH}_{2} \mathrm{O}$ by photosynthesis depends on solar radiation, temperature and the crop water status of the cotton plant. The production of photosynthes is is also directly affected by leaf tissue turgor, as indicated by the dashed line extending from LEAVES to the rate variable PHOTOSYN. Carbohydrates exit the system as a result of maintenance and light respiration. Nitrogen and photosynthates are converted into leaf dry matter by the two rate variables labeled GROWTH, at a rate determined by temperature and the water status of the plant. Thus, plant water status is a determinant of both the supply and demand for metabolites. Water stress reduces the rate of photosynthesis and nitrogen uptake; it also reduces the demand for leaf nutrients through GROWTH. The model also takes into account losses that occur in the value of 


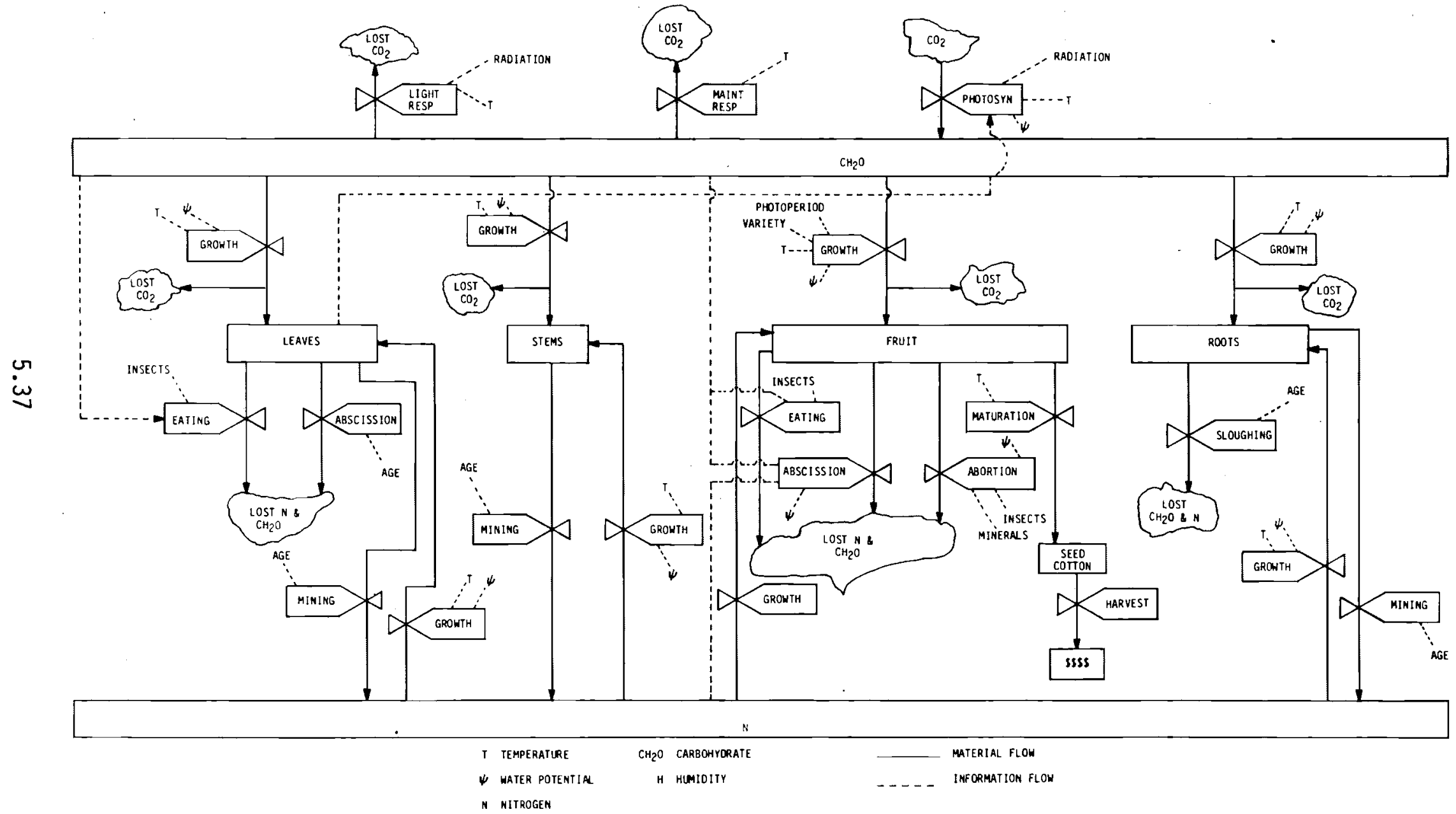

FIGURE 5.2. A Systems Dynamics Conceptualization of the Nitrogen and Carbohydrate Materials Balance in Cotton (Source: Baker et al. 1979) 
the state variable LEAVES due to the effects of insects through the rate variable EATING, which is dependent on the state of $\mathrm{CH}_{2} \mathrm{O}$, through the rate variable ABCISSION which is age-dependent, and through the redistribution of nitrogen to other plant parts through the rate variable MINING which is also age-dependent.

Simulation models differ from statistical crop yield models along almost every dimension that one can use to compare the two approaches. The most obvious differences lie in the relative complexity and size of the two types of models. Statistical crop yield models can usually be represented by a single equation in which the number of variables included $i$ limited by the number of observations in the data set used to estimate the parameters of the mode1. Simulation models, on the other hand, often require several thousand lines of computer code to 1 ist the necessary rate equations, materials balance equations and logic statements needed to accurately simulate major plant growth processes. In addition to this feature, simulation models differ from statistical crop yield models in terms of their more substantial input requirements, which usually consist of daily values of temperature, precipitation and solar radiation, as well as their ability to monitor the status of various plant organs on a daily basis.

Because of their modular structure, simulation models also have the potential to incorporate a much broader range of environmental and technological influences on crop growth processes, including the effect of pests, different methods of cultivation, the addition of plant nutrients and the application of herbicides and insecticides. Moreover, because simulation models are dynamic, they are much better able to capture the differential impact of these phenomena at various stages in the life cycle of the crop being modeled. Finally, and perhaps most importantly, crop simulation models are generalizable to a wide range of environmental conditions. If the basic plant processes embodied in the model are accurately portrayed, then the results obtained from the model should be valid for any set of initial conditions and subsequent changes in the state of the environment that are used in a particular simulation. While the ability to make these models perfectly general is limited to some degree by lack of theory regarding 
certain aspects of plant growth processes, their potential in this area is far greater than in the case of statistical yield models.

Crop simulation modeling is a relatively new field in crop physiology. Table 5.3 represents a partial listing of the modeling efforts that are currently under way. This list was compiled by means of a computer search of all the current records contained in the data bases compiled by the National Agricultural Library (AGRICOLA), the Commonwealth Agricultural Bureaus (CAB) and the U.S. Department of Agriculture (USDA/CRIS). It was supplemented by additional information obtained from conversations with various authors. The list is incomplete for at least two reasons. First, the documentation for many simulation models is often not published in journals or publications covered by available bibliographic and research sources. Secondly, many of the references that are cited as crop simulation models are misleading, usually because the model being referenced turns out not to be a true simulation model of the whole plant. In spite of these problems, the models shown in Table 5.3 probably represent about two-thirds of the true population of models, the majority of which are devoted to cotton, corn, wheat, grain sorghum, soybeans, and alfalfa.

A quick glance at Table 5.3 will also reveal that a good deal of the work in this field is currently being conducted by USDA's Agricultural Research Service (ARS) at two locations, the Blackland Research Center at Temple, Texas and the Boll Weevil Laboratory located at Mississippi State, Mississippi. The models that have either been built or are currently under development by these two groups represent two substantially different approaches to crop simulation. These differences will be examined more carefully in the following section dealing with the methodology of crop simulation.

Crop simulation models, as a group, are not nearly as homogeneous from the standpoint of methodology as are statistical crop yield models. Differences between simulation models tend to fall into two categories, namely: differences in the number of physiological processes that are described mathematically within the models and differences in detail and - complexity accorded to those processes that are included within the models. 
TABLE 5.3. Some Currently Active Crop Modeling Efforts

\begin{tabular}{|c|c|c|c|}
\hline Authors & Institutions & $\begin{array}{r}\text { Mode1 } \\
\text { Name } \\
\end{array}$ & Species \\
\hline $\begin{array}{l}\text { Acock, B., D. N. Baker, and } \\
\text { J. M. Mckinion }\end{array}$ & $\begin{array}{l}\text { USDA/ARS at Mississippi } \\
\text { State }\end{array}$ & GLYSIM & Soybeans \\
\hline Allen, J. and J. H. Stamper & U. of Florida & CITRUSIM & Citrus \\
\hline $\begin{array}{l}\text { Arkin, G. F., J. T. Ritchie, and } \\
\text { R. L. Vanderiip }\end{array}$ & $\begin{array}{l}\text { USDA/ARS at Temple, } \\
\text { Texas, and Kansas State } \\
\text { U. }\end{array}$ & SORG & Sorghum \\
\hline Baker, C. H. and R. D. Horrocks & $\begin{array}{l}\text { Ohio Agr. Res. and } \\
\text { Development Center }\end{array}$ & CORNMOD & Corn \\
\hline $\begin{array}{l}\text { Baker, D. N., J. R. Lambert, and } \\
\text { J. M. Mckinion }\end{array}$ & $\begin{array}{l}\text { USDA/ARS at Mississippi } \\
\text { State, Clemson U. }\end{array}$ & GOSSYM & Cotton \\
\hline Baker, D. N. and J. M. Mckinion & $\begin{array}{l}\text { USDA/ARS at Mississippi } \\
\text { State }\end{array}$ & Unn amed & Wheat \\
\hline $\begin{array}{l}\text { Brown, L. G., J. D. Hesketh, } \\
\text { J. W. Jones, and F. D. Whisler }\end{array}$ & Mississippi State U. & COTCROP & Cotton \\
\hline $\begin{array}{l}\text { Childs, S. W., J. R. Gilley, } \\
\text { and W. E. Splinter }\end{array}$ & U. of Nebraska & Unnamed & Corn \\
\hline $\begin{array}{l}\text { Curry, R. B., G. E. Meyer, } \\
\text { J. G. Streeter, H. J. Mederski, } \\
\text { and A. Eshel }\end{array}$ & $\begin{array}{l}\text { Ohio Agr. Res. \& } \\
\text { Development Center }\end{array}$ & $\begin{array}{l}\text { SOYMOD } \\
\text { OARDC }\end{array}$ & Soybeans \\
\hline Duncan, W. G. & $\begin{array}{l}\text { U. of Florida, U. of } \\
\text { Kentucky }\end{array}$ & SIMAIZ & Corn \\
\hline Duncan, W. G. & $\begin{array}{l}\text { U. of Florida, U. of } \\
\text { Kentucky }\end{array}$ & MIMSOYZ & Soybeans \\
\hline Duncan, W. G. & $\begin{array}{l}\text { U. of Florida, U. of } \\
\text { Kentucky }\end{array}$ & PEANUTZ & Peanuts \\
\hline Fick, G. W. & Cornell University & ALSIM & Alfalfa \\
\hline $\begin{array}{l}\text { Guiterrez, A. P., L. A. Falcon, } \\
\text { W. Loew, P. A. Peipzig, and } \\
\text { R. van den Bosch }\end{array}$ & Unknown & COTSIM & Cotton \\
\hline $\begin{array}{l}\text { Holt, D. A., G. E. Miles, } \\
\text { R. J. Bula, M. M. Schreiber, } \\
\text { D. T. Doughtery, and R. M. } \\
\text { Peart }\end{array}$ & $\begin{array}{l}\text { Purdue University, } \\
\text { USDA/ARS }\end{array}$ & SIMED & Alfalfa \\
\hline
\end{tabular}


TABLE 5.3. (contd)

\begin{tabular}{|c|c|c|c|}
\hline Authors & Institutions & $\begin{array}{r}\text { Mode } 1 \\
\text { Name } \\
\end{array}$ & Species \\
\hline Jackson, B. and B. Hearn & $\begin{array}{l}\text { USDA/ARS at Temple, } \\
\text { Texas and Cotton } \\
\text { Research Unit, } \\
\text { Australia }\end{array}$ & COTTON & Cotton \\
\hline van Keulen, $\mathrm{H}$. & $\begin{array}{l}\text { Nether lands Agr. U., } \\
\text { Wageningen }\end{array}$ & GRORYZA & Rice \\
\hline van Keulen, $\mathrm{H}$. & $\begin{array}{l}\text { Nether lands Agr. U., } \\
\text { Wageningen }\end{array}$ & ARIDCROP & $\begin{array}{l}\text { Natural } \\
\text { vegeta- } \\
\text { tion in } \\
\text { semi- } \\
\text { arid } \\
\text { regions }\end{array}$ \\
\hline Loomis, R. S. and E. Ng & $\begin{array}{l}\text { U. of California, } \\
\text { Davis }\end{array}$ & РОТАТО & Potato \\
\hline $\begin{array}{l}\text { Loom is, R. S., J. L. Wilson, } \\
\text { D. W. Rains, and D. W. Grimes }\end{array}$ & $\begin{array}{l}\text { U. of California, } \\
\text { Davis }\end{array}$ & COTGRO & Cotton \\
\hline $\begin{array}{l}\text { Loomis, R. S., G. W. Fick, } \\
\text { W. A. Williams, W. H. Hunt, } \\
\text { and E. Ng }\end{array}$ & $\begin{array}{l}\text { U. of California } \\
\text { Davis }\end{array}$ & SUBGRO & $\begin{array}{l}\text { Sugar } \\
\text { Beet }\end{array}$ \\
\hline Maas, S. J., and G. F. Arkin & $\begin{array}{l}\text { USDA/ARS at Temple, } \\
\text { Texas }\end{array}$ & SORGF & Sorghum \\
\hline Mass, S. J. and G. F. Arkin & $\begin{array}{l}\text { USDA/ARS at Temple, } \\
\text { Texas }\end{array}$ & TAMW & Wheat \\
\hline Marani, A. & $\begin{array}{l}\text { The Hebrew U. of } \\
\text { Jerusalem }\end{array}$ & ELCOMOD & $\begin{array}{l}\text { Cotton } \\
\text { (Acala) }\end{array}$ \\
\hline $\begin{array}{l}\text { Orwick, P. L., M. M. Schreiber, } \\
\text { and D. A. Hoit }\end{array}$ & Purdue U. & SETSIM & Setaria \\
\hline Reetz, H. & Purdue & PCS & Corn \\
\hline Ritchie, J. T. & $\begin{array}{l}\text { USDA/ARS at Temple, } \\
\text { Texas }\end{array}$ & Unnamed & Corn \\
\hline $\begin{array}{l}\text { Ryle, G. J. A., N. R. } \\
\text { Brockington, C. E. Powell, and } \\
\text { B. Cross }\end{array}$ & $\begin{array}{l}\text { Grassland Research } \\
\text { Institute, Hurley, } \\
\text { Berkshire, England }\end{array}$ & Unnamed & $\begin{array}{l}\text { Uniculum } \\
\text { Barley }\end{array}$ \\
\hline
\end{tabular}


TABLE 5.3. (contd)

\begin{tabular}{|c|c|c|c|}
\hline Authors & Institutions & $\begin{array}{r}\text { Mode } 1 \\
\text { Name } \\
\end{array}$ & Species \\
\hline Staaper, M., and G. F. Arkin & $\begin{array}{l}\text { USDA/ARS at Temple, } \\
\text { Texas }\end{array}$ & CORNF & Corn \\
\hline de Wit, C. T. et al. & $\begin{array}{l}\text { Nether lands Agr. U., } \\
\text { Wagen ingen }\end{array}$ & $\begin{array}{l}\text { PHOTON } \\
\text { and } \\
\text { BACROS }\end{array}$ & Any crop \\
\hline
\end{tabular}

\section{Methodology}

The decision to explicitly exclude certain processes from a simulation model and to simplify the mathematical structure used to describe the processes that remain in a model is usually a conscious one, based not so much on theoretical consideration as it is on the researcher's evaluation of the tradeoffs between the accuracy in prediction that is lost and the cost savings that are achieved by reducing the size of the model. Purists in the field tend to argue that, since the underlying purpose of all crop models is ultimately to predict the response of a crop to changes in exogenous variables, any tradeoff that sacrifices accuracy is difficult to defend (Baker 1981). On the other hand, model builders who opt for the reduction ist approach argue that very large models are impractical for management applications, large area forecasting and climatological assessments (Passioura 1973).

Because this field of crop modeling is a relatively new one, this debate cannot be resolved until a great deal more model development has taken place. However, it is possible to illustrate the methodological differences reflected in these approaches by comparing models that exemplify the two sides of this argument. The large model chosen for this comparison is the cotton simulation GOSSYM (Baker et al. 1981) developed at the Boll Weevil Laboratory by a group headed by D. N. Baker. This group is now developing two additional simulation mdels for wheat and soybeans which incorporate many of the features contained in GOSSYM. The second model to be used in the comparison is CORNF (Staaper and Arkin 1980) a corn simulation model developed by M. Staaper and G. F. Arkin at the Blackland Research Center in Temple, Texas. Under the best 
of circumstances, the ideal comparison would be between two models of the same crop. This was the original plan of this report. However, the documentation for the model, COTTON, that was to be used in the comparison is available only in a draft whose contents are restricted for citation purposes. In spite of this problem, the structure of CORNF is consistent with a modeling philosophy shared by the authors of COTTON and one which is reflected in all of the models developed by this group.

The most obvious difference between the two models is their size. The source code for GOSSYM (currently available only in a draft version) runs over 2800 lines. The comparable length of the source code for CORNF is just 657 lines. While both models are run iteratively on a day-by-day bas is over the respective growing seasons of the two crops, the differences in the core memory space required to load the two models and their respective run times are substantial. In spite of the differences in the size of the two models, the input requirements of the two models are not substantially different.

Input variables common to both models include daily values for solar radiation, maximum and minimum air temperature, and the amount of rainfall or irrigation. Other common inputs include plant population, row spacing and the latitude of the location for which the simulation is being conducted. Differences in the type of inputs required by these two models are relatively minor, with the exception that GOSSYM has the ability to simulate the effects of preseason and side dress applications of nitrogen, different types of cultivation practices and vehicular traffic--all of which require additional input values. The fact that GOSSYM takes these factors into account suggests that the model has a wider range of management applications than does CORNF, although the value of including the impact of different management practices on crop yields many not be important in climatic assessment.

The real differences between the two models lie in the way in which the two models treat some of the major physiological processes which determine crop yield. The following discussion will examine the differences between the two models based on the simulation of photosynthesis, dry matter partitioning and growth and, finally, morphogenesis. 
Photosynthesis. In GOSSYM photosynthes is is simulated in the subroutine PNET. Canopy photosynthesis is an extremely difficult phenomenon to simulate on a dynamic basis (Baker et a1. 1978). The approach taken in PNET is largely empirical, based on crop canopy experiments reported by Baker and Hesketh (1969) and Baker et al. (1972). PNET consists of 48 lines of code. It uses a photosynthetic light response curve for canopy photosynthes is under ideal conditions to compute gross daily photosynthate production as a function of incident solar radiation. This value is then adjusted for plant population, light interception, and moisture stress in order to arrive at a daily value for gross photosynthate production on a per plant basis. Next, PNET calculates respiration losses for both light and maintenance respiration, and subtracts these losses from the daily values of photosynthate production. A final adjustment is made for losses that occur due to growth respiration, and the remainder represents the day's increment of carbohydrate available for distribution among the growing points of the plant.

The photosynthes is routine in CORNF, PHOTO, is about a third the length of PNET. It is basically a two equation model. Intercepted photosynthetically active radiation is computed first using an equation to describe the attenuation of light through a crop canopy. The amount of above ground dry matter produced daily per plant is then calculated as a constant fraction of intercepted solar radiation, adjusted for soil surface area and water stress. The proportionality factor relating dry matter production to interrupted solar radiation is constant unit the plant reaches anthesis, after which it takes on a smaller value due to the aging of leaves. The water stress coefficient is applied when the root zone contains less than $40 \%$ of the potential plant extractable moisture.

Dry Matter Partitioning and Growth. Dry matter partitioning in GOSSYM is simulated in the subroutine GROWTH which consists of approximately 300 lines of code. The data base used to develop GROWTH was obtained from the phytotron experiments of Hesketh et al. (1972) and Bruce and Romkens (1965). In GROWTH, the plant is first inventoried on a daily basis in order to determine a maximum potential growth rate for each of the organs (squares, bolls, leaves, stems 
and roots) as a function of temperature and plant water status. This is done separately for both day and night time periods. GROWTH then sums these requirements and calculates a carbohydrate supply/demand ratio as follows:

$$
\begin{aligned}
& \text { CPOOL = PN + RESC } \\
& \text { CSTRES + CPOOL } / C D
\end{aligned}
$$

where CPOOL represents the total supply of carbohydrate, PN is the value of the day's photosynthate production obtained from PNET, RESC represents carbon reserves carried over from previous days, $C D$ is the total potential carbohydrate demand for the day and CSTRES is the carbohydrate supply/demand ratio.

Next, GROWTH calls the nitrogen budget subroutine, NITRO, which calculates the amount of nitrogen required to assimilate the potential carbohydrate demands of each of the plant organs. The nitrogen requirements are sumed for the vegetative and fruiting parts of the plant, separately, and nitrogen supply/demand ratios are then calculated for the two groups. If either of the supply demand ratios is less than unity the resulting fraction is multiplied by the value of CSTRES to relfect the impact of nitrogen supply limitations on the carbohydrate supply/demand ratio. Finally, the actual growth of each plant organ during the day is computed as the product of its potential growth times the appropriate nitrogen-adjusted carbohydrate supply/demand ratio.

In CORNF, the term growth refers to the increase in weight of the total plant and the ear. Partitioning of dry matter to the vegetative parts of the plant is not simulated. Growth, as defined above, is simulated entirely in the subroutine EAR, which consists of 60 lines of code. Daily grain production is calculated as the product of a kernel growth rate times the number of accumulated daily heat units times the number of kernels per ear. Daily heat units are accumulated in the subroutine HFUNC. The number of kernels is a function of plant dry weight at anthesis and is reduced by a 
water stress coefficient. If daily dry matter production plus reserves, from PHOTO, is insufficient to meet the demand for photosynthate, daily grain production is set equal to the available supply of photosynthate.

Morphogenesis. There are dramatic differences in the ways that the two models simulate morphogenesis. These are due in part to the fact that cotton, unlike corn, is an indeterminant species which can switch back and forth between its vegetative and fruiting stages, while the phenological events in the life cycle of corn occur in strict sequence. Thus, it seems fair to argue that the simulation of morphogenesis in cotton will always require more complex treatment than will the simulation of this process for corn. This is reflected in the structure of the PLTMAP subroutine contained in GOSSYM which literally builds the plant one node at a time, from the first square on. Individual events in the life cycle of the plant such as the development of new nodes and fruiting sites are determined by the state of the plant in relation to previous events, running average temperatures and physiological delays which occur due to the combined effect of nitrogen and carbohydrate stresses calculated in GROWTH and NITRO. PLTMAP simulates the abortion of fruit based on physiological stresses using a seven day lag between the occurrence of stress and the actual abscission of the fruit. The development sequence, which occurs on a daily basis, is terminated at harvest.

Morphogenesis is simulated in the subroutine STAGE of CORNF in four stages: establishment, vegetative, pollination and grain filling. With the exception of tassle initiation, the timing and duration of each stage is dependent on accumulated heat units, adjusted for photoperiod, from HFUNC. Tassle initiation is handled somewhat differently. Leaves are initiated in STAGE based on accumulated heat units, and the total number of leaves is then used to determine the average daylength from emergence to tassle initiation for nine different maturity classes. This last convention in the model is highly empirical and the authors note that its use is questionable, since it does not appear to provide an accurate simulation of tassle initiation over a wide range of environments. 
Validation. To date, GOSSYM has undergone three different validation tests. Fye et al. (1981) used GOSSYM to simulate cotton growth for two years in four different locations in Arizona. Marani et al. (1981) validated Gossym against 57 Acala cotton crops at 19 locations in Israel. Finally, Reddy (1981) used GOSSYM to simulate the growth of 15 cotton crops at four different locations in Mississippi and Arizona. These data are not yet widely available and have not been subjected to a great deal of statistical analysis. However, the RMSE calculated from the Reddy data was approximately 61 kilograms per hectare $(\mathrm{kg} / \mathrm{ha})$ compared to an observed average yield of $949 \mathrm{~kg} / \mathrm{ha}$. Thus, the RMSE associated with these 15 validation trials is approximately $6 \%$ of average yield. CORNF has also been extensively validated against data sets obtained for nine different locations in Texas and the corn belt. The authors report a coefficient determination $\left(r^{2}\right)$ of 0.68 for the relationship between observed and predicted grain yields. Very few of the data used to validate CORNF are contained in the documentation for that model. However, an analys is of 18 different validation trials conducted for crops in Bushland, Texas and Ames, Iowa suggests that the model must simulate yields in some regions much better than in others. The $r^{2}$ values for observed versus predicted yields at these two locations were quite $10 w--0.45$ for Bushland and 0.42 for Ames.

\section{Evaluation}

At the current time, it is probably safe to say that the full potential of crop simulation models has yet to be realized. GOSSYM is a mature simulation model with over 10 years of development behind it. Even so, it is undergoing additional revisions to improve its accuracy and expand the applications for which it can be used. CORNF, on the other hand, is a much newer model, about three years old. Thus, it is still too early to say if the difference between the accuracy of these two models is due to differences in the complexity of their mathematical structures or their respective stages of development. At this time it is believed that GOSSYM could be used very effectively in a climatic and environmental assessment while CORNF could not. Other simulation models for different crops will have to be evaluated on a 
case-by-case basis in order to assess their value for making environmental and climatic assessments of the impact of increases in the ambient concentration of $\mathrm{CO}_{2}$ on crop yields.

A comparison of crop simulation models with alternative approaches according to the six criteria defined earlier in this chapter suggest the following conclusions:

Scope of Environmental Effects. As a group, crop simulation models are designed primarily to simulate the effect of changes in the values of daily meteorological variables on crop development and growth. Incorporating the effect of increases in the atmospheric concentration of $\mathrm{CO}_{2}$ on the production of dry matter within a simulation model can only be done in specific cases if the model already has a photosynthes is subroutine. Even then, experimental data will have to be collected; model refinements will have to be made to explicitly incorporate $\mathrm{CO}_{2}$ variability in the atmosphere; and existing parameters will have to be adjusted, along with new ones, in order to validate the model. Such a process is currently under way in connection with the development of the soybean model GLYSIM. The ability of individual models to capture the impacts of $\mathrm{CO}_{2}$-induced changes in pest abundance will depend upon the way in which morphogenes is is treated within the model. The growth and development of specific plant parts directly affected by pests must be incorporated into the model structure. In that case, plant-specific pest subroutines can be added to the model. This could be done fairly easily with a model like GOSSYM, but only with great difficulty (or perhaps not at all) in the case of a model like CORNF. In spite of these difficulties, simulation modeling still represents the best available method for examining the joint impact of first- and second-order environmental effects on crop yields.

Geographic Portability. Because crop simulation models attempt to portray the response of the whole plant to exogenous changes in the environment, in theory the location where these changes take place should not affect the accuracy of a model. This is the ideal. In the case of GOSSYM, validation trials with data sets obtained from a number of different climatic 
regions suggest that the model is highly portable. CORNF, in the other hand, is not. A recent review (Larsen 1981) of two wheat simulation models developed by the same unit as CORNF point to similar difficulties.

Meteorological Generality. This feature is closely related to the issue of portability and, in theory, crop simuation models should do equally well in both areas. Unfortunately, what little information is available about the validation of crop simulation models does not address this problem in a statistical framework. Fye et al. (1981) in their validation of GOSSYM note that the model does not perform well under conditions of extreme soil moisture stress. Staaper and Arkin (1980) in the ir discussion of the validation of CORNF do not address this problem directly. Larsen (1981) in his revew of wheat models does not address this question directly either. However, he does note that the two models are sensitive to changes in the values of different combinations of meteorological variables. This is a structural feature of all crop simultion models: some parameters tend to dominate over others, such that, under certain conditions, very small changes in the value of an exogenous variable can cause extreme under- or over-shoot in model predictions. This tends to be more of a problem in small models rather than large ones (Loomis et al. 1979). However, large models run the risk of being too insensitive to meteorological extremes.

Incorporation of Technology. Adding the effect of technology means increasing the size of the model. However, due to the modular construction of crop simulation models there are no inherent limitations in this area. GOSSYM and CORNF represent two different extremes with respect to the inclusion of managed inputs. CORNF takes into account only row spacing, plant population, and genotype. GOSSYM, on the other hand, is sensitive to all of these factors plus the quantity and timing of nitrogen applications, tillage practice and vehicular traffic. A distinction is also made in GOSSYM between water that enters the root zone as precipitation or as a result of irrigation treatments.

Data Requirements and Availability. This is a very critical area for these models in terms of their potential values for climatic assessment. The meteorological inputs for crop simulation models can be satisfied by data 
collected at Class $A$ weather stations. However, the problem is not so much the availability of data as it is the need for masses of simulated data. Designing climate scenarios on a day-by-day basis will be an extremely difficult undertaking because it will require transforming assumptions about very general types of climate change into daily joint distributions of solar radiation, temperature and precipitation. Simulating these distributions on a computer will then require running each crop model at least hundreds and very likely thousands of times to obtain estimates for the parameters of the distributions of yields for even a small number of scenarios.

Statistical Accuracy. In most cases, existing crop simulation models have not been subjected to vigorous validation on a systematic basis. This is not to say that individual researchers have failed to check the accuracy of their models by comparing predicted with observed yields from independent data sets. This has been done in almost all cases. However, with the exception of the review of wheat models by Larsen, validation trials have generally been lacking in the area of rigorous statistical analysis. The Research Division of the Statistical Reporting Service in USDA is currently involved in an effort to do just this, and the report by Larsen is just the first of what will hopefully prove to be an exhaustive analysis of a great many crop simulation models. However, based on the available evidence it is impossible to say, for example, whether the current generation of simulation models as a whole actually do a better job of predicting crop yield models than do statistical yield models. In the case of at least one crop, wheat, it would appear that the statistical model developed by Feyerherm and Paulsen is a better predictor of yields that the two wheat simulation models reviewed by Larsen.

\subsubsection{Model Sumary}

The ideal method for determining the effects of $\mathrm{CO}_{2}$-induced environmental changes on crop yields would contain the following characteristics:

1. it would be readily applicable to all major crops;

2. it would be able to capture the effect of all kinds of first-and second-order environmental effects on crop yields; 
3. it would be able to simulate the effects of many different types of climate change, including changes in the variability of climate;

4. the accuracy of the forecasts using existing data sets would be high and invariant to locational changes and to meteorological extremes;

5. it would be able to incorporate the effects of technological change and the use of managed inputs in production.

Unfortunately, no single approach reviewed in this report fits all of these requirements. Each approach has its own individual strengths and weaknesses. A brief sumary of the advantages and disadvantages of the three approaches is shown in Table 5.4 .

Pane 1 Survey Method

The advantages of this approach are that it is readily applicable to many different region-crop combinations and that it can be used to simulate the effects of many different kinds of climate change in a fairly rigorous statistical framework. It has been demonstrated that this approach can be used to separate out the effects of meteorological influences on crop yields from those due to technological change. The disadvantages of th is approach are that the relationship between regional crop yields and meteorological variables is based on personal judgments; the number of meteorological variables that can be utilized is probably limited to three or four at the very most; and that it would be extremely difficult, if not impossible, to extend the analysis to include the joint effects of all of the different kinds of first- and second-order effects.

\section{Statistical Crop Yield Models}

While the crop coverage of these models is uneven, there are no theoretical reasons why the methods that have proven fairly effective in modeling the yield response of wheat, corn and soybeans cannot be extended to other crops in order to provide more systematic coverage. Other advantages of these models include the ability to simulate different types of climate change. Newer models may also be able to account for variations in yield due 
TABLE 5.4. Summary of Crop Yield Model Characteristics

\section{Evaluation Criteria}

Scope of Environmental Effects

Geographic Portability

Meteorologica 1

ir

$$
\text { Generality }
$$

Incorporation of Techno logy

Data Requirements and Availability

Statistical Accuracy

Panel Survey $\quad$ Model Type

Primarily climate-related impacts

Excellent, limited only by data availability

\section{Limited by the number} of variables that can be used

Limited to long-run techno logical change

Uses available meteorological data on precip. and temp.

Fair-good
Primarily climate-related impacts

Results are generally specific to a given region; newer models are more general

Models do not generally perform well under meteorological extremes

Long-run technological change and some managed inputs

Uses recorded or computed values for precip., temp., soil moisture and evapotranspiration by month or phenological stage

Generally good with in range of yield and meteorological variability in data base

\section{Simulation}

Potential to extend to all types of impacts

Excellent, limited only by data availability

\section{Potentially unlimited}

Potential to extend to a large number of managed inputs

Uses daily values for precip., temp., solar radiation soil moisture and evapotranspiration from class $A$ weather stations

Poor to excellent depending on model 
to pest-related episodal events, genotype, cultivation practies and nitrogen applications. The disadvantages of this approach are, first of all, that it is poorly suited to the job of examining the effect of $\mathrm{CO}_{2}$ enrichment on crop yields and, secondly, that the accuracy of these models tends to vary on a location-by-location basis and is also questionable when the models are required to predict yields during years characterized by extreme meteorological conditions. Recent developments in the modeling of wheat suggest that these models can be designed to predict crop yields more accurately under a much wider range of local envirnmental and meteorological conditions. However, this has yet to be demonstrated on a systematic basis.

\section{Crop Simulation Models}

The theory that underlies the simulation of crop growth and development suggests that these models have the potential to meet all of the requirements of the ideal approach outlined above. The realities of crop simulation modeling fall somewhat short of this goal. The first problem is that, while on paper, the coverage of these models is fairly extensive, the accuracy of individual models varies tremendously depending on the complexity of the conventions used to simulate different physiological processes and the amount of development that has gone into the model. A second problem is that while these models represent the most sophisticated, and perhaps the only real means of accurately taking into account the joint impacts of different types of first- and second-order environmental effects on crop yields, substantial research, modification and validation will be required to realize the potential of these models in this area. Finally, the large data requirements of these models, in combination with their size in comparison to statistical yield models, means that simulating the effects of many different types of climate change will not only require the development of a methodology to systematically translate very general statements about climate change into daily inputs of solar radiation, temperature and precipitation; it will also entail substantial costs in conducting the required computer runs to develop the yield distributions consistent with the input distributions of meteorological variables. Thus, while these models represent the only available means of predicting how complex changes in the environment caused 
directly and indirectly by increases in the atmospheric concentration of $\mathrm{CO}_{2}$ will affect crop yields, problems of accuracy and practicality probably will limit their application to environmental assessments of a small number of crops.

Three firlal issues must be sumarized before moving on to the formal discussion of economic models contained with in the report. The first of these issues relates to the lack of congruity between the information that can now be provided by state-of-the-art general circulation models (GCMs) and the information required to drive the different types of crop yield models discussed in this chapter. Throughout this report, it will be argued that much can be learned about the potential range of economic impacts that will occur as a result of elevated $\mathrm{CO}_{2}$ concentrations by exploring how sensitive the economy is to hypothetical $\mathrm{CO}_{2}$-induced changes in the environment. This type of approach does not require extremely accurate information about the magnitude and timing of possible future $\mathrm{CO}_{2}$-induced environmental changes. This is due to the fact that the purpose of such a sensitivity analysis is not so much to forecast the possible direct and indirect economic impacts of $\mathrm{CO}_{2}$-induced environmental changes as it is to examine how sensitive the economy will be to a range of environmental changes that is consistent with current information. In the final analysis, however, one objective of the research that is conducted as a part of the $\mathrm{CO}_{2}$ and $\mathrm{Cl}$ imate Program must be to provide information about $\mathrm{CO}_{2}$-induced climate change that will be compatable with the input requirements of at least one of the different approaches to modeling crop yields.

The extent of current mismatches between state-of-the-art general circulation models (GCMs) and the three methods discussed in this.chapter is shown in Table 5.5. The coverage of this table is limited to five meteorological variables--precipitation, temperature, solar radiation, soil moisture and evapotranspiration. Not all of the different methods for modeling crop yields make use of all of these inputs. The panel survey approach, as utilized in the NDU study discussed earlier in this chapter, requires information on precipitation and temperature, only. Simulation models, on the other hand, require all of these inputs, while most 
TABLE 5.5. Meteorological Data(a) Mismatches Between General Circulation and Crop Yield Models

\begin{tabular}{|c|c|c|c|c|}
\hline & & \multicolumn{3}{|c|}{ Inputs } \\
\hline Data Characteristic & GCM Output & Panel Survey & Statistical & Simulation \\
\hline Geography & Idealized & Rea 1 & Rea 1 & Real \\
\hline $\begin{array}{l}\text { Regional } \\
\text { Delineation }\end{array}$ & 50 grid & $\begin{array}{l}\text { Country or } \\
\text { region (i.e, } \\
\text { state) }\end{array}$ & $\begin{array}{l}\text { State, or } \\
\text { crop report- } \\
\text { ing district }\end{array}$ & $\begin{array}{l}\text { Field level, } \\
\text { but can be } \\
\text { generalized to } \\
\text { crop reporting } \\
\text { district or state }\end{array}$ \\
\hline Frequency & $\begin{array}{l}\text { Annual/ } \\
\text { seasona } 1\end{array}$ & $\begin{array}{l}\text { Average grow- } \\
\text { ing season } \\
\text { values }\end{array}$ & $\begin{array}{l}\text { Monthly } \\
\text { values }\end{array}$ & Daily values \\
\hline
\end{tabular}

(a) Common variables include precipitation, temperature, soil moisture, evapotranspiration and solar radiation.

state-of-the-art statistical yield models use all of these inputs, except solar radiation.

All of these inputs are provided in one form or another by GCMs. However, Table 5.5 illustrates that there are at least three areas in which there are potential mismatches between the information provided by these models and the input requirements of different types of crop yield models. The first of these mismatches is that the geographic domain over which changes in these variables are simulated is idealized. Actual geographic features of existing land masses (i.e., the rockies in Colorado) are not present within these models. Thus, the relationship between the simulated climate in a specific region of the world is, at best, strained.

The second mismatch relates to the consistency between the spatial resolution of GCMs and the different types of crop yield models. The current resolution of GCMs is on the order of a 5 degree square grid--about 350 miles square at the equator, or roughly the size of the state of Colorado. On the surface, at least, climate information that is provided at this level of geographic resolution can be used to make yield projections by means of the 
panel survey method and, in many cases, statistical yield models. While crop simulation models are designed to be field specific in terms of their input requirements, the results obtained from them can be generalized to any geographic scale that would be consistent with the current resolution of GCMs. However, the apparent compatability between the spatial resolution of GCMs and crop yield models is somewhat misleading. First of all, yields for a single crop vary substantially by location. Much of this variation is due to climatic factors. As a result, the smaller the spatial unit used to model crop yields, the smaller will be the deviations between the average yield of a crop in that area and the yields obtained at different locations in that area. This means that yield forecasts that are produced on the bas is of, say, a $1^{0}$ degree square grid will generally be as, or more accurate than, forecasts that are based on a $5^{\circ}$ grid. Whether the $5^{\circ}$ grid can be used as an acceptable lower limit on the spatial resolution of yield forecasts is largely an empirical issue, the answer to which will require more detailed statistical analys is of the results of existing crop yield models.

Increasing the accuracy of a crop yield model by reducing the size of the area for which it will be used to generate forecasts is one thing. Increasing the accuracy of yield forecasts that are used specifically in conjunction with the meteorological information provided by a GCM is a more difficult issue. This is because the change in the accuracy of the yield forecast, as measured by the change in the variance of the forecast error, will also depend on any changes in the accuracy of the meteorological information that is provided by the input source at the smaller scale. The accuracy of GCMs, in general, will be taken up in greater detail shortly. For the moment, it will be sufficient to note that the current consensus within the scientific community is that the accuracy of the climate information that is produced at the $5^{\circ}$ grid scale is poor (National Research Council 1976). This suggests that, until the accuracy of these models can be improved at the $5^{0}$ grid scale, no practical advantage will be achieved by improving the geographic resolution of crop yield models.

The third mismatch relates to the frequency of the climate information produced by GCMs. Traditionally, these models have provided climate 
information on an annual basis. This is compatable only with the panel survey method. Newer models have been constructed that permit climate simulations on a seasonal basis (Wetherald and Manabe 1981), for which the period of model integration is 23 days. While this information is compatable with most statistical yield models (but not crop simulation models), the real problem lies once again in the accuracy of the $c l$ imate information that is provided over 23 day intervals. Until improvements can be made to these models in their ability to forecast seasonal changes in climate, any climate-yield experiments that are conducted using GCM-generated information will have to rely on annual data.

The second issue that needs to be addressed, and one that has been alluded to above, is the question of accuracy. How accurate must the climate information that is provided by GCMs before it can be used effectively to forecast the effects of a $\mathrm{CO}_{2}$-induced climate change on crop yields? The question is clearly rhetorical because the answer depends strictly on the confidence level which the decison maker wants to use in bounding the forecast values for crop yields. What is at issue here, really, is not the choice of a confidence level per se, but rather how to apply that criteria to the yield forecasts that are produced. This is a problem, because there currently exists no method for determining the variances of the forecast errors for the climate variables that represent the output of GCM-based climate forecasts. The reason for this is that, since GCMs predict climate changes for an. idealized geographic domain, there is no way of validating the model results based on climate data from an actual location.

In the absence of a method for validating the forecasts produced by GCMs, the accuracy of these forecasts cannot be measured statistically in the same way that this can be done for statistical crop yield models and simulation models. As a result, it is impossible to determine, quantitatively, how accurate the yield forecasts will be that are produced using GCM-generated climate data. This suggests very strongly that a fairly high priority ought to be $\mathrm{placed}$ on developing GCMs that can be validated using existing climate data bases. Until that can be done, it will not be possible to determine the accuracy of yield forecasts by any method which uses the information produced by GCMs as a basis for calculating crop yields. 
The final issue of concern relates to the consistency between the information that is produced by crop yield models and the input requirements of agricultural sector models. Economic assessment of the impacts of $\mathrm{CO}_{2}$-induced environmental changes will require information about crop yields that is compatible with the crop coverage and regional delineation contained in the agricultural sector model used in the next stage of the analysis. At the current time, it does not appear that any of the methods reviewed in this chapter could be put to such a task without additional model development. The problem lies not in the capability of crop yield to provide information about possible yield changes, but rather in the crop and regional coverage of existing models. To satisfy the requirement for multi-regional forecasts for many different crops first requires the selection of an agricultural sector model in order to determine which region-crop combinations must be included in the overall modeling framework. Once that is decided, then there are basically three alternatives, two of which relate directly to the methods presented in his chapter. The first alternative involves selecting one of the three methods and developing all future yield forecasts based on its use. The second alternative would be to draw from all of the available methods on a crop-by-crop basis, which would necessarily involve establishing statistical criteria for selecting the most accurate models and then subjecting the available candidate models to these criteria.

The final alternative, which will be discussed in greater detail in the next chapter, would involve embedding a set of statistical yield models within the framework of an agricultural sector model. This last approach would eliminate the need for separate yield and economic models, and it would also reduce the need to employ the assumption of the independence of environmental effects on crop production which almost assuredly would have to be invoked in cases where different yield and agricultural sector models are used. It should be noted, however, that these advantages would be purchased at the expense of meteorological generality and, perhaps, accuracy if projected meteorological changes are outside the range of variability contained in the data bases used to estimate the meteorological parameters of the yield equations within the agricultural sector model. 


\section{REFERENCES}

Anderson, J. R. et al. 1977. Agricultural Decision Analysis. University of Iowa Press, Ames, Iowa.

Anderson, R. et al. 1977. Mode ls Designed to Efficiently Allocate Irrigation Water Use Based on Crop Response to Soil Mo isture Stress. Environmental Resources Center, U.S. Department of Agriculture, Fort Collins, Colorado.

Baier,W. 1973. "Crop Weather Analys is Model; Review and Model Development." Journal of Applied Meteorology 12(6):937-947.

Baker, D. N. 1981a. "Development and Validation of Crop Simulation Models." MS. Crop Simulation Unit, Agricultural Research Service, U.S. Department of Agriculture, Mississippi State, Mississippi.

Baker, D. N. 1981b. GOSSYM: A Simulator of Cotton Growth and Yield. Crop Simulation Unit, Agricultural Research Service, U.S. Department of Agriculture, Mississi.ppi State, Mississippi.

Baker, D. N. et al. 1972. "The Simulation of Growth and Yield in Cotton: I. Gross Photosynthesis, Respiration and Growth." Crop Science 12(4):431-435.

Baker, D. N. et al. 1978. "Crop Architecture in Relation to Yield." In Crop Physiology, ed. V.S. Gupta, Oxford and I.B.H. Company, London, England.

Baker, D. N. et al. 1979. "Plant Response to Environmental Conditions and Modeling Plant Development." In Proceedings of the Weather and Agricultural Symposium, ed. Wayne Decker, pp. 69-135. University of Missouri at Columbia, Columbia, Missouri.

Benci, J. F. and E. C. A. Runge. 1975. "Modeling Corn Production under Variable Soil Climatic Conditions." In Proceedings of the 30th Annual Corn and Sorghum Research Conference, pp. 194-214. American Seed Trade Association, Washington, D.C.

Berndt, E. R. and L. R. Christensen. 1973. "The Internal Structure of Functional Relationships: Separability, Substitution and Aggregation." Review of Economic Studies 40(3):403-410.

Borch, K. 1968. The Economics of Uncertainty. Princeton University Press, Princeton, New Jersey.

Bruce, R. R. and M. J. M. Romkins. 1965. "Fruiting and Growth Characteristics of Cotton in Relation to So il Mo isture Tension." Agronomy Journal $57(1): 135-140$.

Cross, H. Z. and M. S. Zuber. 1972. "Prediction of Flowering Dates in Maize Based on Different Methods of Estimating Thermal Units." Agronomy Journal $64(3): 351-355$. 
Dugas, W. A. et a1. 1981. "Evaluating Relationships Between Weather Data Network Distribution and Simulated Sorghum Yields." Paper Presented at the 15 th Conference on Agriculture and Forest Meteorology, Apri1 1-3, 1981, Anahe im, California.

Feyerherm, A. M. 1979. Estimation of Wheat Yields from Meteorological and Agronomic Observations. Prepared for NASA/Johnson Space Center, Earth Observation Division, Houston, Texas.

Feyerherm, A. M. and G. M. Paulsen. 1981. "Development of a Wheat Yield Prediction Mode 1." Agronomy Journal 73(w):277-282.

Fye et a1. 1981. "The Validation of GOSSYM: Arizona Conditions." MS. Crop Simulation Unit, Agricultural Research Service, U.S. Department of Agricultural, Mississippi State, Mississippi.

Halter, A. N. and G. W. Dean. 1965. "Use of Simulation in Evaluating Management Strategies Under Uncertainty." Journal of Farm Economics 47(3):557-73.

Hanway, J. J. 1971. "How a Corn Plant Develops." Special Report No. 48, Iowa State University - Cooperative Extension Service, Ames Iowa.

Haun, J. R. 1974. "Prediction of Spring Wheat Yields from Temperature and Precipitation Data." Agronomy Journal 66(4):405-409.

Hesketh et a1. 1972. "Simulation of Growth and Yield in Cotton: II. Environmental Control of Morphogenesis." Crop Science 14(4):541-546.

Hundtoft, E. B. et al. 1971. "Non-Linear Least Squares Methods of Estimating Physical Constants in Intermediate Relationships." Transactions of American Society of Agricultural Engineers 14(4):750-756.

Kunreuther, H. 1972. Risk-Taking and Farmers' Crop Growing Decisions. Report No. 7219, University of Chicago Center for Mathematics and the Study of Business Economics, Chicago, Illinois.

Larsen, G. A. 1981. Progress Report on the Evaluation of Plant Growth Models. Research Division, statistical Reporting Service, U.S. Department of Agriculture.

Loomis, R. S. et al. 1979. "Explanatory Models in Crop Physiology." Annual Review of Plant Physiology $30(4): 339-367$.

Marani, A. D. and D. N. Baker. 1981. Development of a Predictive Dynamic Simulation Model of Growth and Yield in Acala Cotton. Israel Binational Foundation, Tel-Aviv, Israel.

McQuigg J. D. 1975. Economic Impacts of Weather Variability. Department of Atmospheric Sciences, University of Missouri at Columbia, Columbia, Missouri. 
National Defense University. 1978. Climate Change to the Year 2000: A Survey of Expect Opinion. U.S. Government Printing Office, Washington, D.C.

National Defense University. 1980. Crop Yields and Climate Change to the Year 2000. 2 Vols, U.S. Government Printing Office, Washington, D.C.

Nelson, W. L. and R. F. Dale. 1978a. "A Methodology for Testing the Accuracy of Yield Predictions from Weather-Yield Regression Models for Corn. Agronomy Journal 70(3):734-740.

Nelson, W. L. and R. F. Dale. 1978b. "Effect of Trend or Technology Variables and Record Period on Prediction of Corn Yields with Weather Variables." Meteorology 16(1)215-222.

Nield, R. E. and N. H. Richman. 1979. Simulation Studies of Corn HybridCl imate Response in Nebraska. Research Bulletin No. 287. The Agricultural Experiment Station, Institute of Agricultural and Natural Resources, Lincoln, Nebraska.

Nield, R. E. and M. W. Seeley. 1976. "Applications of Growing Degree Days in Field Corn Production." In Agrometeorology of the Maize Crop, pp. 426-236. Wor ld Meteorological Organization Publication No. 481, World Meteorological Organization, Geneva, Switzerland.

Nield, R. E. and M. W. Seeley. 1977. "Growing Degree Day Predictions for Corn and Sorghumn Development and Some Applications for Crop Production in Nebraska." Research Bullet in 280. The Agricultural Experiment Station, Institute of Agricultural and Natural Resources, Lincoln, Nebraska.

Neild, R. E. et al. 1979. "Impacts of Different Types of Temperature Change on the Growing Season for Maize." Agricultural Meteorology 20(3)367-374.

Passioura, J. B. 1973. "Sense and Nonsense in Crop Simulation." Journal of the Australian Institute for Agricultural Science 39(1):181-183.

Ramirez, J. M. and A. Bauer. 1973. "A Small Grains Response to Growing Degree Day Units." Paper presented at the American Society of Agronomists Annual Meeting, Nov. 11-19, Las Vegas, Nevada.

Robertson, G. W. 1968. "A Biometeorological Time Scale for a Cereal Crop, Involving Day and $\mathrm{Night}$ Temperatures and Photo-period. International Journal of Biometeorology 12(3):191-223.

Robertson, G. W. 1974. "Wheat Yields for 50 Years at Swift Current, Saskatchewan, in Relation to Weather." Canadian Journal of Plant Science 54(4):625-650.

Reddy, V.R. 1981. "Validation of the Cotton Simulation Model GOSSYM." Ph.D. Thesis, Mississippi State University, Starkville, Mississippi.

Shaw, R. H. 1974. "A Weighted Moisture-Stress Index for Corn in Iowa." Iowa State Journal of Research 49(2)Pt. 1:101-114. 
Staaper, M. and G. F. Arkin. 1980. CORNF: A Dynamic Growth and Development Model for Maize. Texas Agricultural Experiment Station, Blackland Research Center, Temple, Texas.

Stuff, R. G. et al. 1979. "Status of Yield Estimation Technology." In Proceedings of Technical Session, Vol. II, the LACIE Symposium, NASA Johnson Space Center., Houston, Texas.

Thompson, L. M. 1969. "Weather and Technology in the Production of Wheat in the Limited States." Journal of Soil and Water Conservation 24(1):219-224.

Thompson, T.L. 1981. "Current Agricultural Management Programs with Climate as a Factors." In The Economic Impact of $\mathrm{Cl}$ imate on Food Production, VIII, pp. 39-49, Oklahoma Climatological Survey, University of Oklahoma, Norman, Oklahoma.

Wetherald, R. T. and S. Manabe. 1981. "Difference of Seasonal Variation Upon the Sensitivity of a Climate Model." Journal of Geophysical Research 86 (C2): 1194-1204. 


\subsection{ANALYSIS OF METHODS AND MODELS FOR ASSESSING THE DIRECT ECONOMIC IMPACTS OF $\mathrm{CO}_{2}$-INDUCED ENVIRONMENTAL CHANGES ON THE AGRICULTURAL SECTOR OF THE U.S. ECONOMY}

The preceding chapter of this report contained an analysis of the methods that can be used to quantify the possible impacts of $\mathrm{CO}_{2}$-induced environmental changes on crop yields. This chapter will focus on the methods that are available for evaluating the impact of these yield changes on the total production and market prices of many different agricultural commodities, as well as the profits which farm operators receive as a result of producing and marketing these goods. In that context, this chapter is organized around three basic objectives. The first of these is to identify the salient features of a generic climate assessment methodology that must be taken into consideration when adapting agricultural sector models to forecast the economic impacts of $\mathrm{CO}_{2}$-induced environmental changes. The second objective of this chapter is to develop a set of criteria that can be used to evaluate alternative types of agricultural sector models, given the demands imposed upon them within an environmental assessment framework. The final objective of this chapter is to assess alternative types of agricultural sector models based on these criteria and to make recommendations regarding the development of specific models for evaluating the economic impacts of $\mathrm{CO}_{2}$-induced environmental changes on the agricultural sector of the U.S. economy.

This chapter is divided into three main sections. Section 6.1 contains a discussion of the methodological issues associated with adapting agricultural sector models for assessing the long-term economic impacts due to foreseeable changes in the ambient concentration of $\mathrm{CO}_{2}$. This discussion will, in turn, provide the basis for developing a set of evaluation criteria that can be used to assess the relative strengths and weaknesses of alternative modeling approaches. Section 6.2 examines the basic differences between positive (econometric) and normative (mathematical programing) models of the agricultural sector with reference to the evaluation criteria developed in the previous section. In addition, this section of the report contains 
recommendations regarding the necessary changes to selected agricultural sector models in order to make them better suited to assessing the economic impacts of $\mathrm{CO}_{2}$-induced environmental changes. Finally, Section 6.3 will summarize the strengths and weaknesses of the models reviewed in previous sections and make specific recommendations regarding the use of selected models in future research.

\subsection{METHODOLOGICAL ISSUES IN ASSESSING THE DIRECT ECONOMIC IMPACTS OF $\underline{C O}_{2}$-INDUCED ENVIRONMENTAL CHANGES}

There appear to be very few precedents in the literatire of assessing the economic impacts of long-term changes in the environment. Those studies that have been done in this area (Ben-David and Schulze 1975; Schmitz et al. 1975; Mayo and McMillan 1975) have generally focused on the effect of changes in climate upon a single crop in isolation from the productive opportunities afforded by competing crops. In addition, none of these studies have attempted to isolate the impact of changes in the mean values of meteorological variables upon agricultural production from the impact of changes in climate variability. Nor have any of them looked directly at the influence of climate variability of agricultural production as a result of risk. Finally, with the exception of the study by Ben-David and Schulze, no attempt was made to simulate the effects of $c l$ imate change on the agricultural sector on a dynamic basis. While the model employed by Ben-David and Schulze was explicitly dynamic in this regard, changes in the mean values of meteorological variables over time were assumed to occur at a single, constant rate. The assumption of a constant rate of change is probably safe enough in the absence of more complete information; however, no attempt was made to deal with the uncertainty surrounding that estimate, through sensitivity analysis or more explicit stochastic methods.

Some of the shortcomings noted above are relatively easy to correct. For example, including additional commodities in this type of analys is can be achieved at the cost of increasing model size, but not necessarily model 
sophistication. 0ther shortcomings are more difficult to remedy. For example, developing a model that can simulate the impact of $\mathrm{CO}_{2}$-induced environmental changes on a stochastic bas is involves the application of fairly sophisticated methodological techniques, particularly if one plans to take into account the effect of changes in climate variability over time on risk-related aspects of agricultural production. It is also often true that refinements that are made to a model to enhance a particular aspect of the analysis involve serious tradeoffs with other areas of the analysis. For example, building a dynamic structure into a model in order to capture the effects of lags between the occurrence of an event and its economic consequences makes it possible to simulate the adjustment of the agricultural sector to exogenous changes in the environment over time. However, this feature is purchased at a relatively large cost if one plans to conduct the analysis on a stochastic basis.

Unfortunately, methodological sophistication can always be justified for its own sake, of ten without prior regard to its cost or consequences in terms of the overall research strategy that is ultimately developed. Therefore, in examining criteria that can be used to evaluate alternative modeling approaches, it is very important to keep in mind not only the additional benefits that are purchased by adopting a particular refinement to existing methods and models, but also the tradeoffs that may be involved in other areas of the analysis. In that general context, Section 6.1.1 will outline a set of alternative strategies for using agricultural sector models to provide information about the future economic impacts of $\mathrm{CO}_{2}$-induced environmental changes. Sections 6.1.2 to 6.1.7 contain a general discussion of the rationale for including a specific set of evaluation criteria and some of the general problems that may arise in applying these criteria to specific models in the framework of these strategies.

The criteria used in this chapter to evaluate agricultural sector models will include: 
- ability to model dynamic adjustments in the agricultural sector due to lags between the occurrence of an event and its economic consequences,

- ability to model the effects of $\mathrm{CO}_{2}$-induced environmental changes in a stochastic framework,

- adequacy of the level of the regional and commodity disaggregation contained within the model,

- adequacy of model treatment of linkages to the rest of the world through impacts and exports,

- data needs and availability,

- degree of statistical accuracy and model stability.

\subsubsection{Alternative Strategies for Direct Impact Analysis}

Current estimates suggest that the concentration of $\mathrm{CO}_{2}$ in the earth's atmosphere will double in approximately the next 50 to 100 years, assuming no remedial measures are taken to reduce carbon emissions to the atmosphere due to fossil fuel combustion. While there are no practical problems strictly associated with making very long-term forecasts on this scale, the numbers that fall out of this kind of analysis must be regarded with a great deal of skepticism. The problem is not so much a methodological one as it is one of economic theory: there is no reason to believe that a model which can generate a very accurate forecast of agriculatural commodity output and prices over a period of, say, a year will provide a more accurate forecast of these variables 100 years from now than will a model that produces less precise short-term forecasts. The reason for this is that, historically, the impact of changes in consumer preferences and technology on the supply and demand for all kinds of goods and services has tended to be quite profound. Unfortunately, economic theory provides little if any guidance in explaining how these types of changes occur. While some effort has been made to explain long-term trends in the bias of technological change, for example the transition from a labor-intensive to a capital-intensive economy (Hayami and Ruttan 1971; Binswanger et al. 1978), long-term changes in both technology and preferences are generally treated as exogenous influences on economic 
systems. In that general framework, the issue of forecast accuracy is somewhat academic. Consequently, efforts to "forecast" the impacts of $\mathrm{CO}_{2}$-induced environmental changes on any sector of the economy should not be taken too literally.

Given the theoretical problems with using economic models to make long-term predictions, two strategies come to mind which could be useful in determining how changes in the ambient concentration of $\mathrm{CO}_{2}$ will affect the agricultural sector of the U.S. economy. These two strategies can be distinguished on the basis of whether $\mathrm{CO}_{2}$-induced yield changes and their resulting effects on the economic behavior of producers and consumers are treated as occurring instantaneously or more slowly over time.

Instantaneous Adjustment Strategy

Actual changes in the ambient concentration of $\mathrm{CO}_{2}$ will occur gradually over time. It is just as likely that the impact of these changes on crop yields will also occur fairly gradually, albeit in some cases with slight lags. Nevertheless, it is possible to argue that the actual adjustments that take place within the agricultural sector to $\mathrm{CO}_{2}$-induced yield changes will be influenced substantially by exogenous factors which cannot be predicted. If that is the case, then simulating the dynamic adjustment of the agricultural sector to gradual changes in crop yields may not add very much to the analysis. Using this set of assumptions, it would be a relatively trivial matter to simulate the single period adjustment of the agricultural sector to different crop yield scenarios using almost any off-the-shelf agricultural sector model. If the model were initialized on current economic and agricultural conditions, employing this strategy would be identical to simulating the immediate effect of $\mathrm{CO}_{2}$-induced yield changes on taday's agricultural economy.

While it is not altogether clear that this would necessarily be true under all foreseeable circumstances, it is still possible to argue that this type of strategy would be consistent with a "worst case" economic scenario. Such a claim can be justified on two grounds. The first is that, given sufficient time and economic incentives, technological changes can be expected to occur which will enable agricultural producers to reduce the adverse 
effects and take advantage of the favorable effects of foreseeable changes in the ambient concentration of $\mathrm{CO}_{2}$ on crop yields. The second is that, over long enough periods of time, land, labor and capital are much more responsive to own and cross price changes than they are when the period of adjustment is short. This is the underlying distinction between the short-run and long-run in the theory of production. In the long-run, the factors of production are assumed to be highly mobile and this permits producers to take advantage of a wider set of productive opportunities than is possible over shorter periods of adjustment.

It is also not clear that the agricultural sector is totally incapable of making substantial adjustments to fairly large, albeit hypothetical, changes in crop yields that might occur very suddenly in a number of different regions. The use of this strategy could be improved by employing a dynamic model to simulate the adjustments that would occur over a decade or so after the occurrence of such a shock to the system. Presumably, the ability of the agricultural sector to adjust to such a shock today will not be as great as it will in the future, particularly if there is sufficient warning about the nature of the changes that will occur. Thus, the underlying purpose of developing a "worst case" economic scenario is not to show how much worse off producers and consumers will be, but rather to try to determine if they might not be better off than some voices in the scientific community would lead us to expect.

The strategy described above makes no pretense to simulate the actual economic impacts of $\mathrm{CO}_{2}$-induced yield changes. The value of this strategy, as suggested above, is that it may be a useful and inexpensive approach in deciding whether or not increases in the ambient concentration of $\mathrm{CO}_{2}$ are likely to cause severe enough adverse impacts in the agricultural sector to warrant further analysis using more sophisticated methods. This last statement must be qualified because the conclusions that can be drawn from this approach are not symetric with respect to the types of normative assessments that can be made about the impact of $\mathrm{CO}_{2}$-induced yield changes on the agricultural sector. If the worst case indicates that there will be an 
increase or only a small decline in the sum of consumer and producer surplus, there are grounds for arguing that the long-term impacts of $\mathrm{CO}_{2}$-induced yield changes are more likely than not to be beneficial. On the other hand, if there is a substantial decrease in net benefits it would not be possible to make any specific statements about how much better off society would be in the long run, given the presumably counteracting influences of technological change and greater mobility of the factors of the production.

\section{Dynamic Adjustment Strategy}

Virtually all economic models of the agricultural sector are dynamically recursive. This means that the values of some endogenous variables that are determined within the model during period $t$ are used as exogenous variables in the model during subsequent periods. The use of lagged variables in agricultural sector models will be discussed in more detail in Sections 6.1.2 and 6.2.1. For the moment, the significance of this feature in agricultural sector models is that it permits one to simulate the economic impacts of $\mathrm{CO}_{2}$-induced yield changes on a gradual basis over time. The projections that are made using such an approach will still be based on current technological trends. Predicted adjustments in the agricultural sector will also be constrained by the relative fixity of the factors of production embedded within the parmeters contained in the model that is used. The advantages of this approach over the static strategy are as follows:

- By taking into account the rate at which $\mathrm{CO}_{2}$-induced yield changes will occur, it will give some indication of the timing of the economic impacts of these changes.

- Comparisons can be made between the timing and magnitude of the economic impacts due to increases in the ambient concentration of $\mathrm{CO}_{2}$ and the timing and magnitude of the economic impacts associated with different forecasts for exogenous variables in the mode1, such as per capita incomes and the aggregate price leve1, and with other types of exogenous "shocks" to the agricultural sector.

- Finally, adjustments can be made in model parameters to reflect different assumptions about future changes in technology and the 
mobility of resources to see how these differences would effect the timing and magnitude of the economic impacts due to gradual $\mathrm{CO}_{2}$-induced yield changes.

The essential features of the dynamic adjustment strategy, consistent with the advantages just mentioned, can best be stated in terms of the following three stage process. The initial stage would consist of establishing a base case scenario, which typically involves developing a set of forecasts for the exogenous variables that appear in the model from the initial period to some future period. The number of variables for which base case forecasts must be developed depends upon the individual model. The time span chosen for the simulation will be dependent upon the best available projections of the time it will take for the ambient concentration of $\mathrm{CO}_{2}$ to reach some pre-specified level which is of direct policy concern. In the current case, the uncertainty associated with making very long-term forecasts for exogenous variables may require the development of a number of such forecasts, each containing a different set of assumptions about long-term trends in the aggregate price level, per capita income and other relevant exogenous variables. The resulting set of simulations that are performed using these forecasts represents an attempt to show what the future may look like in the absence of any increase in the ambient concentration of $\mathrm{CO}_{2}$.

The next stage involves determining the direct impacts associated with superimposing upon each of the base cases a "representative" $\mathrm{CO}_{2}$-induced yield scenario. Assuming for the moment that the analysis is not stochastic, this will involve taking the best available estimates of the expected effects of $\mathrm{CO}_{2}$ on the average yields of selected key crops, presumably on a regional basis, and introducing these yield changes into the model, on a . period-by-period basis, at a rate that is consistent with the available wisdom on the subject. This would be repeated for each of the base cases. By comparing these simulations with the base case simulations one can then isolate the impacts on the agricultural sector due solely to the $\mathrm{CO}_{2}$-induced yield changes embedded within the representative scenario.

The third stage of the dynamic adjustment strategy consists of sensitivity analysis, which involves changing the values' of either certain 
exogenous variables in the model or else model parameters in order to determine how sensitive the agricultural sector is to the assumptions associated with these changes. In the current framework, this type of analys is can be used, first of all, to determine the extent to which the different assumptions that are built into the base case scenarios affect the economic impacts that occur as a result of the representative $\mathrm{CO}_{2}$ scenario. A second type sensitivity analysis consists of examining the differential impacts associated with altering both the magnitude of $\mathrm{CO}_{2}$-induced yield changes and the rates at which these changes occur over time. This approach represents a fairly simple way of taking into account the uncertainty associated with the estimates contained in the representative yield scenario. A final type of sensitivity analysis involves changing selected parameters within the agricultural sector model to reflect assumptions regarding the impact of technological change on crop yields and the greater responsiveness of land, labor and capital to changes in their own and cross prices, given a long enough period of time in which to adjust. The purpose of performing this type of analysis is to try to more nearly duplicate the long-run conditions under which adjustments to $\mathrm{CO}_{2}$-induced yield changes will actually be made. However, a great deal of caution must be exercised in doing this, particularly in econometric models, since the sensitivity of a model to changes in certain parameters may be misleading due to errors in misspecifying the model or using an incorrect estimation procedure.

The advantages of the dynamic adjustment strategy have already been discussed, and lie primarily in the areas of sensitivity analysis and the ability to provide some indication of how the impacts of $\mathrm{CO}_{2}$-induced yield changes will occur over time. The major disadvantage of this strategy is that is is necessary to execute a large number of computer runs in order to analyze even a fairly limited number of combinations of scenarios, each of which involves different assumptions about the values that exogenous variables and variables of policy interest will take on over time. Therefore, in making use of sensitivity analysis it is important to keep the number of assumptions that one makes about expected changes in both kinds of variables relatively small. 


\subsubsection{Dynamic Adjustments in Economic Analys is}

In economics, the impact of a certain event on the behavior of consumers and producers may not be immediate. For example, an increase in the price of corn that occurs in the winter cannot affect corn acreage until the next spring when the crop is planted. Lags such as this between the occurrence of an event and the effect that it has on the behavior of economic agents are a very common phenomenon. Some lags are relatively short, as in the example above, but in many cases the effects of an economic cause may be spread over many years. Lags that affect the production and consumption of goods and services may arise for three types of reasons. The first of these is technological which may involve ignorance on the part of consumers and producers about the existence of substitute goods and services or, in the case of producers, lack of experience with a recent technological innovation. Lags due to technology also arise because of the extremely high costs associated with adjusting the stock of durable goods in response to price changes over short periods of time. Finally, in the context of agricultural production, technological lags exist because producers cannot adjust their planting decisions for at least a season, and usually a year, once made. The second reason for lags is institutional. Institutional lags in agricultural production are quite common and are due to constraints that are imposed upon producers due to contractual arrangements in marketing, imperfections in capital markets which make it more difficult for small producers to borrow money, and finally by some government programs which involve contractual arrangements that may prohibit more optimal adjustments to commodity price changes. The final type of lag in economics is due to the effects of uncertainty, usually about future commodity prices, such that consumers and producers may either fall back on habit or else make the assumption that price changes will only be temporary. Lags of this sort are sometimes referred to as phsychological lags (Ner love 1958).

The exact manner in which these types of lags can be built into economic models of the agricultural sector will be discussed in Section 6.2. What all of these approaches share in common is that, by making variables in the current period depend upon the values of these variables in previous periods, 
it is possible to simulate the lag-related adjustments over time. Since changes in $\mathrm{CO}_{2}$ will occur gradually, it may be possible to shed more light on the way in which the agricultural sector will respond to $\mathrm{CO}_{2}$-induced yield changes by making use of dynamic models in any future analys is of the direct economic impacts of these yield changes. However, one serious problem with the use of these models is that the adjustment mechanisms that are built into them usually involve either quarterly or, at most, annual lags. This is consistent with the traditional use of these models in providing short-term forecasts of commodity output and prices. However, over very long periods of time, the significance of short-term lagged adjustments within the agricultural sector will probably be overwhelmed by those adjustments which take place over a much longer time span. Presumably, it is the ability of the agricultural sector to make the latter $k$ ind of adjustment that must be reflected in the lag structure used in a model to evaluate the economic impacts of $\mathrm{CO}_{2}$-induced yield changes. However, in order to do this, at least within the framework of an econometric model, would unquestionably require developing a new model with appropriately long intervals built into its 1 ag structure (say 5 or 10 years) and then estimating the parameters of the model on an extremely long, and perhaps inadequate, time series data base, drawn from census records.

\subsubsection{Modeling the Impact of Climate Variability on the Agricultural Sector}

Whenever very long range forecasts are contemplated the question of how to best deal with uncertainty about the future must be addressed. In the current framework, there are three different sources of uncertainty which will surround any attempt to forecast the economic impacts of $\mathrm{CO}_{2}$-induced yield changes. These include: (1) uncertainty about the values which exogenous variables in any model will take on over time, (2) uncertainty about the effects of $\mathrm{CO}_{2}$-induced environmental changes on crop yields, and (3) uncertainty about the rate at which $\mathrm{CO}_{2}$-induced yield changes will occur over time.

Of the three, the first area is the least tractable, largely because the variables are themselves the outcome of economic forces at work in other sectors of the economy. While it might be possible to obtain future values 
for these variables from simulations conducted using macro-models of the whole economy, the usual practice in this area is to construct alternative scenarios that are consistent with a range of expectations regarding the values which exogenous variables will take on in the future. In either case, the real focus of concern is not so much to predict the future, as it is to determine how sensitive the impacts associated with changes in policy-related variables are to changes in the values of exogenous variables. This approach can never replace stochastic analysis, but given the large amount of uncertainty associated with macroeconomic changes within the agricultural sector, there is really no other alternative. Therefore, the remainder of this section will examine alternative ways of introducing into the analysis the uncertainty that exists regarding the timing and magnitude of $\mathrm{CO}_{2}$-induced yield change.

In Section $3.2^{\text {iof }}$ this report, four different kinds of environmental effects on crop yields due to changes in the ambient concentration of $\mathrm{CO}_{2}$ were discussed. Two of these are random, because of the intervening effect of $\mathrm{CO}_{2}$ on climate in one case, and, in the other, because of the additional intervening effect of climate on pests. Through either of these two paths, it is due to the influence of climate variability that the effects of $\mathrm{CO}_{2}$-induced yield changes become random. Consequently, changes within the agricultural sector in the equilibrium levels of output, prices and acreage which are in part determined by crop yields will also be random. Furthermore, the randomness conferred on these variables will in turn be passed along to any we if are measures used in the analysis such as producer and consumer surplus. Thus, virtually all of the economic impacts of $\mathrm{CO}_{2}$-induced environmental changes on the agricultural sector that are likely to be of interest can be thought of as random.

There are at least three different ways of treating the intervening effect of climate variability on the economic impacts of $\mathrm{CO}_{2}$-induced environmental changes in the agricultural sector. Two of these depend on the availability of information about the parameters of the crop yield distributions used in the analysis. The first method, which involves the use of sensitivity analysis, does not require this information. However, it does require subjective estimates of the variability in yields about their expected 
mean values. Once this determination is made, it is a simple matter to produce a series of simulations that are consistent with the range of yield estimates chosen. The major drawback to this method is the large number of simulations that would be required to conduct this analys is if an attempt was made to account for the variability in each crop in each region. A more practical approach would be to vary the yield of all crops proportionally from their respective means by several different values, and then conduct the sensitivity analys is based on these deviations. This would provide useful information about how sensitive the agricultural sector will be to moderate and extreme deviations in crop yields from their expected values.

The second approach that can be used involves Monte Carlo simulation (Hammersley and Handscomb 1964), which requires estimates of (a) the starting and terminal values of the mean and variance of the yield for each region-crop combination and (b) changes in the values of these parameters that occur over time as a result of increases in the ambient concentration of $\mathrm{CO}_{2}$. The next step involves constructing sampling distributions based on this information for each region-crop combination in each year over which the simulation is to be conducted. This task is not as complicated as it sounds if one assumes that changes in these two parameters of the yield distributions will occur at a constant rate. For example, assume that the distribution of the ith crop in the jth region in the recent past is found to be normally distributed with a mean of $\mu_{i j 1}$ and a variance of $\sigma_{i j 1}^{2}$. (The subscript $t=1$ denotes that these are the starting period parameters of that distribution). Now assume that the application of crop yield modeling techniques reveals that with a constant rate of $\mathrm{CO}_{2}$ buildup' in the atmosphere, the terminal parameters of this distribution for $t=100$ will be $\mu_{i j 100}, \sigma_{i j 100}^{2}$. Using this information one can can then find the rate constants a and b such that $\mu_{i j 1}(1+a)^{100}=\mu_{i j 100}$ and $\sigma_{i j 1}^{2}(1+b)^{100}=\sigma_{i j 100}^{2}$. Determining the values for $\mu_{i j t}$ and $\sigma_{i j t}^{2}$ in intervening years is computationally trivial. If $a$ and $b$ are assumed to be stochastic, such that each of these two rates are characterized by a distribution for which subjective estimates of the mean and variance can be obtained, calculating $\mu_{i j t}$ and $\sigma_{i j t}^{2}$ is more complicated, but still mathematically 
straightforward. Nevertheless, if there are 50 region-crop combinations and the forecast period is 100 years, Monte Carlo simulation will still require the construction of 5000 sampling distributions.

Once these distributions are constructed, a series of simulation runs are conducted, for each of which it is necessary to select a random yield value for each region-crop combination, in each period, from the appropriate sampling distribution. In the case above, this would mean 5000 different yield vlaues per simulation. The number of individual simulations that must be conducted depends upon the degree of error that one is willing to accept in two different areas: (1) obtaining interval estimates for the mean values of variables that are of policy interest (i.e., equilibrium prices and output and consumer and producer surplus), and (2) making statements about the probability that individual values for these variables will be above or below a specific value. Other things being equal, fewer simulations must be conducted to perform the first kind of analysis than the second, since the latter involves making statements about the areas under the tails of the distributions of the relevant model outputs. Ultimately, the determination of how many simulations can be made using standard sampling theory, although this choice will also depend on the size of one's research budget.

The use of Monte Carlo simulation also raises problems in modeling the impact of climate variability on the behavior of producers due to risk. Methods for incorporating risk into agricultural sector models will be discussed in more detail in Section 6.2. However, most of these methods explicitly or implicitly involve the introduction of terms reflecting the expected values and variances (and, in some cases, the covariances) of either commodity prices or net returns per acre in the acreage response equations for each of the commodities in the model. If the underlying distributions of commodity prices are assumed to be stable and the model is used in a short-term forecasting framework, then it is possible to assume that these risk terms will be exogenous to the agricultural sector and to treat them accordingly in the simulation. In the current framework, however, this approach is difficult to justify. In each period of the simulation, any 
change in the sampled values for crop yields will also affect comodity prices and net returns per acre. Changes in the periodic values of these variables will, in turn, influence their expected values and variances on which the allocation of acreage by producers to different commodities will depend. This means that risk terms, if they are to be included at all, will have to be treated as endogenously determined variables within the model so that their values can be updated at each iteration, with appropriate lags to reflect the delay in adjusting to the changes in the values of the risk variables.

Monte Carlo simulation can become extremely expensive depending upon: (1) the number of random variables that are included in the model, (2) the number of time periods that are contained in the forecast, and (3) the size and the complexity of the simulation model being used. Thus, from a practical standpoint, the use of this method is probably a realistic alternative in the framework of the instantaneous adjustment strategy, but not the dynamic adjustment strategy.

A third approach that can be used for stochastic analysis involves the application of fairly recent advances in modern control and systems theory in order to calculate the expected values and variances of each of the endogenous variables in a dynamic model (Anstrom 1970). The theory that underlies this approach is complex and the method is not widely used in economic analysis because of the requirement that the simulation model be linear. Even then, the calculation of the final forecasting error variances for each endogenous variable that is of policy interest is not a trivial matter. The requirement that the mode 1 be linear precludes the use of most currently available econometric models of the agricultural sector. However, a strong justification can be made for either re-estimating the nonlinear equations in these models on a linear basis, or else constructing an entirely linear model from the ground up based on the argument that (1) the accuracy of model used to make short-term forecasts is an inappropriate criteria for evaluating its accuracy over long periods of time, and (2) simple, linear models often do a fairly good job of forecasting long-term economic trends, at least on an ex ante basis (Pindyke and Rubinfeld 1976). If that is the case, then this approach would merit serious attention for further research. 


\subsubsection{Regional and Cormodity Disaggregation in Agricultural Sector Models}

It is unlikely that the joint effects of future increases in the ambient concentration of $\mathrm{CO}_{2}$ on the natural and physical environments in which agricultural production takes place will be evenly distributed across space. Substantial differences in regional climates, soils and cultural practices, which exist today, further suggest that the impacts of these environmental changes on the yields of individual crops will be characterized by a high degree of spatial variability.

In that general context, there are two basic problems associated with using a national model to assess the economic impacts of $\mathrm{CO}_{2}$-induced environmental changes upon the agricultural sector. First of all, employing a national model in this framework would make it extremely difficult to introduce the effect of regional variations in the impacts of $\mathrm{CO}_{2}$-induced environmental changes on crop yields into the analysis. While it might be possible to work around this problem by developing an appropriate weighting scheme to reflect regional differences in the anticipated effects of $\mathrm{CO}_{2}$-induced environmental changes on the yields of individual crops, there is a second and more serious problem with the use of national models. The crux of this problem is that national models portray the technology of agricultural production in a highly aggregated fashion that masks the heterogeneity that actually exists in the agricultural sector at the regional level and, beneath that, at the level of the individual firm.

Over very short periods of time, not more than a year or two, aggregation errors that exist in national models will probably not have a profound effect on forecast accuracy. This is due in large part to the fact that, even though substantial exogenous shocks to the agricultural sector may occur, the adjustment by producers and consumers to these shocks is constrained in the short-term by technological and institutional rigidities in the economic system and by force of habit. In the long-run, however, producers and consumers will be able to adjust to these shocks and, in the process, substantial interregional shifts in production can occur. When this happens, the geographic mix of cultural conditions and the climates to which individual 
crops are exposed, as well as the regional mix of technologies used to produce these crops, will also change. This means that the representative environmental conditions and technology that are embedded in the parameters of a national model will no longer be appropriate. As a result, the model will not do a good job of forecasting the aggregate impacts associated with full adjustment. Since these are the very same type of changes that are likely to be associated with the economic impacts of increases in the ambient concentration of $\mathrm{CO}_{2}$ within the agricultural sector, the use of national models to conduct this analys is is particularly inappropriate.

The selection of the appropriate regional delineation to use in any future analysis is extremely problematic. First of a11, it is important to realize that, short of characterizing the technology of every firm in the agricultural sector, the conditions under which it is possible to treat aggregate producer behavior as if it were the outcome of the decisions of a single profit maximizing producer (e.g., "exact" aggregation) are so stringent that is virtually impossible to avoid problems of aggregation entirely (MacFadden 1978). Bearing this in mind, the choice of an appropriate regional delineation will be guided by the following set of considerations:

1. what is already available in existing models;

2. congruity with existing environmental conditions and technology;

3. congruity with a priori information about the geographic distribution of $\mathrm{CO}_{2}$-induced climate changes; and

4. availability of data consistent with (2) and (3) above.

As one might expect, the regional delineations contained in existing agricultural sector models are largely based on the second and fourth considerations above. Application of the second criteria represents an attempt to increase the degree of homogeneity that characterizes regional production within the model. However, this must in all cases be tempered by the availability of data that is consistent with the regional delineation chosen. Increasing the number of regions in an agricultural sector model in order to achieve greater homogeneity will, in theory, reduce errors due to 
aggregation. However, it will also complicate the problems and expense associated with data gathering, since the depth of readily available agricultural production data is directly related to the size of the geographic unit on which the model is based.

The selection of the appropriate number of commodities to include in an agricultural sector model is directly related to the regional disaggregation scheme adopted. National models generally employ, as a minimum, the following commodities: (1) food grains, including wheat and sometimes rye; (2) feed grains, including corn, oats, barley and sorghum; (3) other important crops, usually including soybeans, cotton and, sometimes, rice and tobacco; (4) livestock, including cattle and calves, sheep and lambs, hogs and dairy products and, sometimes, poultry. The selection is usually based on their contribution to the value of farm production or total acreage.

Introducing a regional dimension into an agricultural sector model involves the consideration of at least three additional factors. The first two of these relate to the competitiveness of crops on a regional as opposed to a national level. For example, although the price of sorghum will influence the amount of corn that is planted at the national level, corn and sorghum do not compete directly in Illinois and Iowa whereas they do in Nebraska and Kansas. Thus the choice of crops to be included in the model will affect the regional delineation that is used. In the case above, for example, a strong argument can be made for separating the high plains from the corn belt in order to better capture the different patterns in the regional competitiveness of these two crops. A related problem involves the inclusion of crops that are grown in only a few states. As the number of regions included in the analysis increases, the impact which changes in the price of regional specialty crops can have on the planting, harvesting and marketing decisions associated with other crops in the region will increase. If the cross price impact is substantial on a regional basis then, in theory, its effect ought to be reflected in the structure of the model. This involves either increasing the number of crops which receive explicit structural treatment within the model, or else introducing the price effect exogenously through one or more parameters. The former approach will increase model size 
substantially, while the latter will increase the number of exogenous variables for which long-term forecasts must be made before model simulations can be performed.

A final problem in selecting an appropriate region-commodity mix is that very dramatic changes in yields due to increases in the ambient concentration of $\mathrm{CO}_{2}$ may cause equally dramatic inter-regional shifts in agricultural production. For example, the results of the NDU study cited in Section 5.2 could be construed to suggest that a large warming scenario, such as might accompany an increase in the ambient concentration of $\mathrm{CO}_{2}$, might extend the northern range of corn and soybeans into major wheat growing regions in the U.S. and Canada. The ability of agricultural models to predict substantial changes in the geographic distribution of crop production will be discussed more fully in Section 6.2. For the moment, it is only important to recognize that any a priori information that can be developed about such changes, if only in the form of alternative hypotheses about the nature of these shifts, will influence the regional and commodity breakdowns that are included in the analysis.

The inter-relationships that exist between the choice of an appropriate regional breakdown and the choice of the number of commodities to include in a model make it difficult to select a scheme that does not involve a very large number of both commodities and regions. That is generally not practical and concessions to theory must generally give way to concessions to practicality and cost. Therefore existing commodity breakdowns that include from 7 to 10 major crops, plus livestock, are probably satisfactory, as long as the regional delineation that is chosen is fairly faithful to the existing pattern of intra-regional and inter-regional competition between crops that currently exists, yet flexible enough to incorporate more dramatic changes in the geographic distribution of agricultural production, if that is possible.

\subsubsection{Linkages to the Rest of the World}

Changes in the ambient concentration of $\mathrm{CO}_{2}$ have the potential to alter the environmental conditions under which crops are raised throughout the world and to alter the competitiveness of individual nations in the trading of 
agricultural commodities. For example, assume that global warming due to an increase in ambient concentration of $\mathrm{CO}_{2}$ was distributed on a geographic bas is such that wheat yields in the southern Great Plains were substantially reduced, while yields in Canada, The Peoples Republic of China and the Soviet Union increased fairly dramatically. Under these conditions, the differential effect of $\mathrm{CO}_{2}$ on wheat production could be substantial enough to alter the current trading pattern in wheat between the U.S. and the rest of the world.

This situation is illustrated in Figure 6.1.A. The initial supply and demand curves for wheat in the U.S. are illustrated by the lines $D_{a} D_{a}^{\prime}$ and $O S_{a}$, while the supply and demand curves for the rest of the world are shown by the lines $D_{b} D_{b}^{\prime}$ and $O S_{b}$. The prevailing trade price for wheat is $P$. At this this price, the U.S. produces $Q_{a}^{S}$ bushels, of which $Q_{a}^{D}$ is consumed at home. The difference between the quantity of wheat produced and the quantity demanded, or $Q_{a}^{S}-Q_{a}^{D}$, is exported to the rest of the world. At that same price, the rest of the world produces $Q_{b}^{S}$ bushels of wheat and consumers $Q_{b}^{D}$ bushels. The difference between supply and demand is satisfied through imports from the U.S. Thus $Q_{a}^{S}-Q_{a}^{D}=Q_{b}^{D}-Q_{b}^{S}$.

The hypothetical effects of $\mathrm{CO}_{2}$ on crop yields in the U.S. and the rest of the world are illustrated in Figure 6.1.B. In the U.S., a decrease in wheat yields in one of its major production regions is indicated by a shift to the right (toward the horizontal axis) in the supply curve for that commodity, from OS $\mathrm{S}_{\mathrm{a}}$ to $\mathrm{OS}_{\mathrm{a}}^{\prime}$. The impact of increased yields in the rest of the world is illustrated by a shift to the right (away from the horizontal axis) of its supply curve, from $0 S_{b}$ to $0 S_{b}^{\prime}$. The result is that the international price of wheat declines from $P$ to $P^{\prime}$. At this new price, the U.S. now becomes an importer of wheat, producing $Q_{a}^{S^{\prime}}$ bushels of wheat and consuming $Q_{a}^{D^{\prime}}$ bushels, the difference being made up by imports from the rest of the world. The rest of the world now becomes a net exporter of wheat, producing $Q_{b}^{S}$ ' bushels of wheat while consuming on $l y Q_{b}^{D^{\prime}}$ bushels and exporting the difference between demand and supply to the U.S. In the process, producer surplus in both regions is much affected: the profits of U.S. producers decline, while the profits enjoyed by producers in the rest of the world increase. However, consumer surplus, as 


\subsection{A Pre- $\mathrm{CO}_{2}$ Trade}

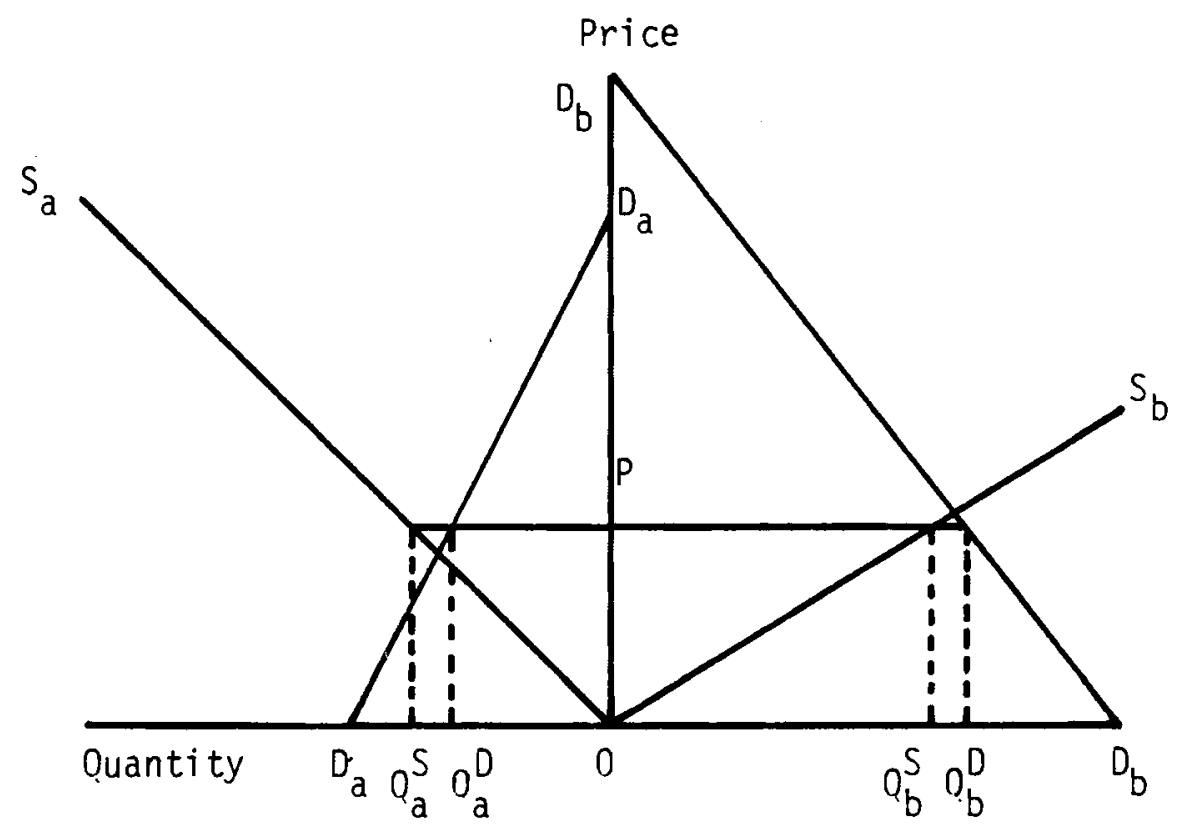

\section{1.b Post $-\mathrm{CO}_{2}$ Trade}

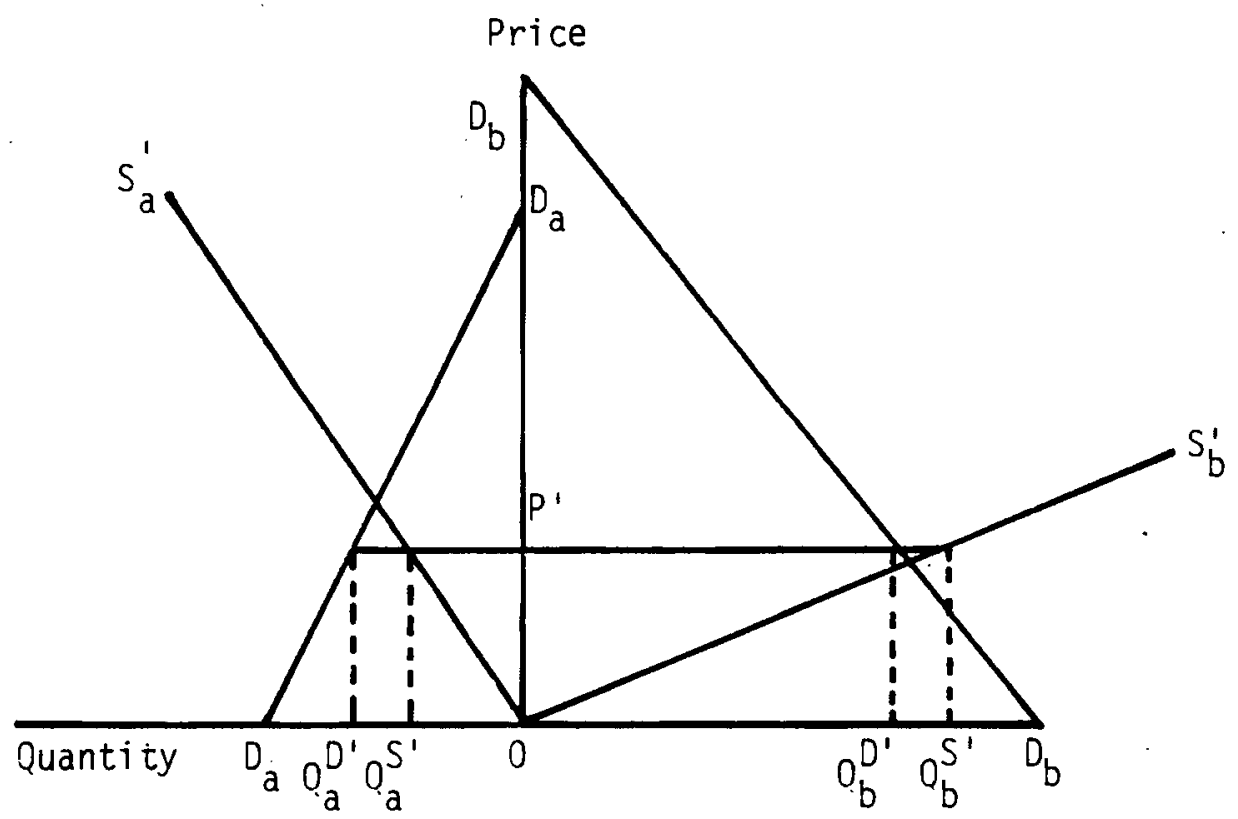

FIGURE 6.1. Hypothetical Effect of $\mathrm{CO}_{2}$ on International Trade 
measured by the area above the price line and below the demand curves for wheat, does not change substantially in either region.

An important point to note in this analysis is that, even though the hypothetical effects of $\mathrm{CO}_{2}$ involve fairly large shifts in the supply curves for both regions, the effect on the equilibrium price of wheat is not very great. This is due, in part, to the counteracting effects of $\mathrm{CO}_{2}$ on the two supply curves. But a more important reason for this is that the demand curves for wheat in the relevant equilibrium price range were purposely drawn to reflect the inelastic nature of the demand for wheat. Thus, while production in each of the two regions changes fairly dramatically as a result of hypothetical $\mathrm{CO}_{2}$-induced yield changes, there is substantially no change in price and a relatively small increase in the total amount of production by the two regions. Moreover, the quantity of wheat demanded by consumers in each of the regions remains about constant; therefore, consumer surplus also remains nearly constant. On the other hand, there is a substantial redistribution of producer surplus (profits) from the U.S. to the rest of the world. Because the demand for most agricultural commodities is price inelastic, the impacts illustrated in Figure 6.1 are not altogether inconsistent with what might happen if the effects of $\mathrm{CO}_{2}$-induced environmental changes on crop yields actually occur as hypothesized here.

Agricultural sector models usually give fairly extensive treatment to export demands, while imports of agricultural commodities are usually treated as exogenous. In general, however, agricultural sector models are not explicitly linked to other regions in the world in a way that would permit the examination of increases in the ambient concentration of $\mathrm{CO}_{2}$ on the production of agricultural commodities in other nations. Typically, this kind of analysis is conducted using models which permit the supply and demand for agricultural commodities in many regions to adjust simultaneously with changes in international commodity prices. These trade models generally do not contain as many commodities as do agricultural sector models. In addition, it is sometimes the case that the commodities that are included in these models represent aggregate groupings; for example, feed grains are sometimes treated as a single aggregate composed of corn, sorghum, oats and barley. Because of 
these problems and the fact that each nation is generally treated as a single regional unit, the use of agricultural trade models in the current context will require aggregating the effect of $\mathrm{CO}_{2}$-induced environmental changes on crop yields across both regions and crops within individual countries.

\subsubsection{Data Needs and Availability}

The most obvious data problem in any future analysis of the effect of increases in the ambient concentration of $\mathrm{CO}_{2}$ on the agricultural sector is the lack of data regarding the future effects of $\mathrm{CO}_{2}$-induced environmental change on crop yields. Methods for generating this information based on assumed changes in the concentration of $\mathrm{CO}_{2}$ and climate have been discussed in the previous chapter. Obviously, without this kind of information no analysis of the economic impacts of these yields will be possible. However, as pointed out in Section 6.1.1, the fact that there may be a great deal of uncertainty surrounding there yield estimates does not mean that it will be impossible to shed some light on how the agricultural sector might respond to hypothetical $\mathrm{CO}_{2}$-induced yield changes. Developing yield scenarios that contain the most extreme assumptions about the nature of $\mathrm{CO}_{2}$-induced yield changes and then examining the response of the agricultural sector to these changes represents an important first step in attempting to gauge whether there really will be a problem or not. Further sensitivity analys is based on systematically altering the magnitude of $\mathrm{CO}_{2}$-induced yield changes and the rates at which these changes may occur can then be useful in demonstrating the conditions under which increases in the ambient concentration of $\mathrm{CO}_{2}$ will lead to substantial changes, up or down, in consumer and producer welfare.

Beyond this issue, the problem of data needs and availability will not become important if existing, off-the-shelf models are to be used in the analysis. On the other hand, the availabilty of data may represent a serious problem if new models are to be developed. This issue will be addressed specifically in Section 6.2 of the report in connection with the desirability of incorporating longer-term lags into econometric models of the agricultural sector in order to better capture the long-run adjustment process likely to be associated with consumer and producer behavior in response to gradual changes in the ambient concentration of $\mathrm{CO}_{2}$ over the next 50 to 100 years. 


\subsubsection{Statistical Accuracy and Stability of Agricultural Sector Models}

The question of statistical accuracy arises only in connection with positive (econometric) models of the agricultural sector. These models are typically used to forecast short-term changes that occur within that sector on a quarterly or annual basis. Normative (mathematical programing) models are designed to show the optimal allocation of resources that would exist within the agricultural sector, consistent with a specific objective function, technology and resource constraints. Thus, positive models attempt to forecast actual changes in relevant economic variables, while normative models show ideal changes against which the result of positive models can be measured.

However, the parameters of econometric models which determine the rate at which acreage and output adjust either recursively or simultaneously in response to commodity price changes are estimated on recent historical data. Thus, as was pointed out in Section 6.1.2, these parameter estimates are really not appropriate for analyzing long-term adjustments that may take 19 or 20 years to work their way through the economy. If this problem is combined with the fact that it is impossible to use parameters that reflect historical rates of technological change to explain future rates, then as suggested earlier, the accuracy of the models used to make these forecasts may not be a concern of high priority at this time. However, an issue that is far from academic in this framework is model stability.

The problem of stability arises whenever a simulation model contains difference equations, i.e., when it is dynamic. In these situations, one must be concerned with the oscillatory behavior of endogenously-determined variables in order to ensure that these oscillations are not explosive. This is particularly true of short-term forecasting models that are to be used in long-term policy analysis, since it is sometimes the case that these models are not stable, but that the explosiveness of the oscillations in the model only become a problem when simulations are conducted over a large number of periods.

The easiest way to look at this problem is by using a simple threeequation model of agricultural production that treats the amount of the com- 
modity supplied in period $t$, or $Q_{t}$, as a function of the price of that good in the previous period, $P_{t-1}$. This model can be expressed linearly by the following demand, supply and market clearing equations:

$$
\begin{array}{ll}
Q_{\mathrm{t}}^{D}=\theta-\Psi P_{\mathrm{t}} & \text { (Demand) } \\
Q_{\mathrm{t}}^{\mathrm{S}}=-\delta+\phi P_{\mathrm{t}-1} & \text { (Supply) } \\
Q_{\mathrm{t}}^{D}=Q_{\mathrm{t}}^{\mathrm{S}} & \text { (Market Equilibrium) }
\end{array}
$$

By substituting Equation (6.1b) and (6.1c) into (6.1a) and shifting the subscripts ahead one time period one obtains the first-order difference equation

$$
P_{t+1}+\frac{\phi}{\psi} P_{t}=\frac{\theta+\delta}{\psi}
$$

The solution to Equation (6.2) is, then

$$
P_{t}=\left(P_{0}-\frac{\theta+\delta}{\Psi+\phi}\right)\left(-\frac{\phi}{\psi}\right)^{t}+\frac{\theta+\delta}{\Psi+\phi}
$$

where $P_{0}$ represents the initial price in the base period. The last expression in Equation (6.2b) represents the intertemporal equilibrium price in the mode $1, \bar{P}$. Substituting $\bar{P}$ back into Equation $(6.2 b)$ allows one to express the time path of price as

$$
P_{t}=\left(P_{0}-\bar{P}\right)\left(-\frac{\phi}{\Psi}\right)^{t}+\bar{P}
$$

The pattern of oscillation in $\mathrm{P}_{\mathrm{t}}$ can be determined by inspecting the complementary function $(-\phi / \psi)$. Since the fundamental axioms of economics. require that demand curves be downward sloping and supply curves upward 
sloping, then $\Psi, \phi>0$. As a result, it can be shown that $P_{t}$ must have an oscillatory time path since $(-\phi / \Psi)$ will alternate between positive and negative values from period to period. The nature of these oscillations will depend on the relative magnitude of $\phi$ with respect to $\Psi$. There are three possibilities: (1) if $\phi>\Psi$, the oscillations will be explosive, and the model will predict negative prices and quantities; (2) if $\phi=\Psi$, the price oscillations will be. uniform over time; and finally (3) if $\phi<\Psi$, then the oscillations in price will be dampened and the model will converge to a steady state solution.

The model represented by Equation (6.1) is used purely for illustrative purposes; however, examining the stability of linear models for which solutions to first-order difference equations can be obtained involves the same type of analysis, albeit on a much larger scale. In models that involve higher-order lags, stability analysis becomes more complicated, involving the examination of the characteristic roots of these higher-order difference equations (Chow 1967; Goldberger 1959). In the case of large, nonlinear dynamic models there is virtually no direct way of determining how stable the model is. Usually, one must test for stability by performing a series of simulations, over different periods of time, and using different time paths for the exogenous variables in the model.

\subsection{ANALYSIS OF AGRICULTURAL SECTOR MODELS}

There are many different ways of classifying agricultural sector models. Basic differences between them can be distinguished on a number of grounds, for example: (1) whether they are dynamic or static; (2) whether they are designed primarily for policy analys is or to forecast agricultural commodity prices and output; (3) whether linkages to the national economy in the model are explicit or implicit; (4) whether the geographic delineation contained in the model is international, national or regional, or so on. These distinctions become very important when selecting a model to fulfill a specific set of research objectives. However, because an individual model may incorporate a great many different attributes, classifying agricultural sector models along multi-dimensional lines often obscures more general differences. 
modity supplied in period $t$, or $Q_{t}$, as a function of the price of that good in the previous period, $P_{t-1}$. This model can be expressed linearly by the following demand, supply and market clearing equations:

$$
\begin{array}{ll}
Q_{t}^{D}=\theta-\Psi P_{t} & \text { (Demand) } \\
Q_{t}^{S}=-\delta+\phi P_{t-1} & \text { (Supply) } \\
Q_{t}^{D}=Q_{t}^{S} & \text { (Market Equilibrium) }
\end{array}
$$

By substituting Equation (6.1b) and (6.1c) into (6.1a) and shifting the subscripts ahead one time period one obtains the first-order difference equation

$$
P_{t+1}+\frac{\phi}{\Psi} P_{t}=\frac{\theta+\delta}{\Psi}
$$

The solution to Equation (6.2) is, then

$$
P_{t}=\left(p_{0}-\frac{\theta+\delta}{\Psi+\phi}\right)\left(-\frac{\phi}{\Psi}\right)^{t}+\frac{\theta+\delta}{\Psi+\phi}
$$

where $P_{0}$ represents the initial price in the base period. The last expression in Equation (6.2b) represents the intertemporal equilibrium price in the mode $1, \bar{P}$. Substituting $\bar{P}$ back into Equation (6.2b) allows one to express the time path of price as

$$
P_{t}=\left(P_{0}-\bar{P}\right)\left(-\frac{\phi}{\Psi}\right)^{t}+\bar{P}
$$

The pattern of oscillation in $P_{t}$ can be determined by inspecting the complementary function $(-\phi / \Psi)$. Since the fundamental axioms of economics. require that demand curves be downward sloping and supply curves upward 
sloping, then $\Psi, \phi>0$. As a result, it can be shown that $P_{t}$ must have an oscillatory time path since $(-\phi / \Psi)$ will alternate between positive and negative values from period to period. The nature of these oscillations will depend on the relative magnitude of $\phi$ with respect to $\Psi$. There are three possibilities: (1) if $\phi>\Psi$, the oscillations will be explosive, and the model will predict negative prices and quantities; (2) if $\phi=\psi$, the price oscillations will be. uniform over time; and finally (3) if $\phi<\Psi$, then the oscillations in price will be dampened and the model will converge to a steady state solution.

The model represented by Equation (6.1) is used purely for illustrative purposes; however, examining the stability of linear models for which solutions to first-order difference equations can be obtained involves the same type of analysis, albeit on a much larger scale. In models that involve higher-order lags, stability analysis becomes more complicated, involving the examination of the characteristic roots of these higher-order difference equations (Chow 1967; Goldberger 1959). In the case of large, nonlinear dynamic models there is virtually no direct way of determining how stable the model is. Usually, one must test for stability by performing a series of simulations, over different periods of time, and using different time paths for the exogenous variables in the model.

\subsection{ANALYSIS OF AGRICULTURAL SECTOR MODELS}

There are many different ways of classifying agricultural sector models. Basic differences between them can be distinguished on a number of grounds, for example: (1) whether they are dynamic or static; (2) whether they are designed primarily for policy analys is or to forecast agricultural commodity prices and output; (3) whether linkages to the national economy in the model are explicit or implicit; (4) whether the geographic delineation contained in the model is international, national or regional, or so on. These distinctions become very important when selecting a model to fulfill a specific set of research objectives. However, because an individual model may incorporate a great many different attributes, classifying agricultural sector models along multi-dimensional lines often obscures more general differences. 
Therefore the distinction between agricultural sector models that is used in this report is perhaps the most basic one of all, namely: between models that are designed to predict the actual behavior of economic agents within the agricultural sector, the so-called positive approach to economic modeling, and those that are constructed in such a way as to show how economic agents should behave in order to achieve a given economic objective, which represents a normative approach.

As was stated earlier, models based on the positive approach are often referred to as econometric models, because they involve the application of econometric methods to the specification of model equations and the estimation of the parameters contained within these equations. Briefly, an econometric model is composed of a series of behaviorial equations, each of which relates a single dependent variable to a set of explanatory, or independent variables. The way in which these equations are organized as a whole, as well as the functional form and explanatory variables that are used for each equation, collectively represent a set of hypotheses about how economic agents behave in the agricultural sector. The parameters of these equations, which determine how sensitive the dependent variable will be to changes in each of the explanatory variables, are estimated statistically by means of advanced multivariate regression techniques (e.g., econometrics) using either historical, time series and/or cross-sectional observations on all of the variables. The accuracy of these models as forecasting instruments can then be tested in much the same manner used to validate statistical crop yield models: by seeing how well the model actually forecasts the behavior of agricultural commodity prices, production and consumption, using a different data set than the one employed to estimate the parameters of the model.

Normative models consist of three quantitative elements: (1) an objective function that is consistent with the aggregate behavior of economic agents, such as the maximization of the sum of producer and consumer surplus or the minimization of variable costs; (2) an alternative set of production activities which describe the various ways in which variable inputs and fixed factors can be combined in order to produce different comodities; and (3) a set of restrictions on the availability of one or more of these inputs, and, 
in the case of cost minimization models a set of demand requirements that must be met. Given these elements, mathematical programming algorithms are then used to allocate the available resources to alternative production activities in the model in order to satisfy the economic criterion that is contained in the objective function. The basic differences between these two approaches from a methodological standpoint is that, whereas the estimation of econometric models involves the use of optimization methods (e.g., least squares and maximum likelihood) in order to obtain the statistical properties of model parameters given the observed values of the endogenous variables in the model, normative models employ optimization methods to solve for the values of these endogenous variables, given explicit values for the technological parameters that appear in the model. It is on the basis of this distinction that the terms positive and normative have traditionally been grounded.

Given a clearer understanding of this basic difference between the two approaches, the remainder of this section is devoted to a more detailed analysis of the structural features of these models, and to developing a set of recommendations regarding the way in which these models could be used to evaluate the economic impacts of $\mathrm{CO}_{2}$-induced yield changes in the agricultural sector, consistent with the modeling strategies and evaluation criteria identified in Section 6.1.

\subsubsection{Econometric Models of the Agricultural Sector}

The original intent of this section of the report was to provide a detailed comparison of the structural features of a representative sample of econometric, agricultural sector models currently being used by agricultural economists in USDA, land grant universities and in the private sector. A list of the models that have been surveyed in the course of this research appears in Table 6.1. Apart from the understandable difficulties in obtaining the documentation to proprietary models in the private sector, it very quickly became clear that virtually all of the models examined undergo constant revision either to improve their performance or to make them more flexible in terms of the different research uses to which they can be put. The 
TABLE 6.1. Agricultural Sector Models Reviewed for this Report

Model Name

Integrated Livestock and Crops Model

General Equilibrium Model of U.S. Agriculture (GEM)

Chase Econometrics Agricultural Model

ISU/NRE LP Econometric Model (CARD Recursive-CR Model)

ISU/NRE National-Regional LP (CARD Tatonnement-CT Model)

National-Interregional Agricultural Projections System (NIRAP)

National Agricultural Policy Simulator (POLYSIM)

World Grains, 0ilseeds and Livestock Model (GOL)

AGSIM

Crops Econometric Model (CROPS)

National Spatial Equilibrium Model of Crop Production
Staff Contact/Organization

Abner Womack, Department of Agricultural Economics, University of Missouri at Columbia

Dean Hughes, Federal Revenue Bank, Kansas City, Missouri

Denn is Steadman, Chase Econometrics, Bala Cynwyd, Pennsylvannia

Wen-Yuang Huang, Iowa State University, Ames, Iowa

Burton English, Iowa State University, Ames, Iowa

David Watt, Department of Agricultural Economics, Michigan State University, East Lansing, Michigan

David Banker, Food and Agricultural Policy Branch National Economic Divison, Economics and Statistics Service, USDA

Karen Liu, Trade and Policy Branch, International Economics Divison, Economics and Statistics Service, USDA

Glen Collins, Department of Agricultural Economics, Texas A\&M University, College Station, Texas

Robert Green, National Economics Division, Economics and Statistics Service, USDA

Robert Taylor, Department of Agricultural Economics, Montana State University, Bozeman, Montana

consequences of this feature of these models are that many existing models are either not well documented, or that the documentation that does exist does not reflect recent, and sometimes substantial, revisions to their basic structure. However, it also became clear that, in spite of this problem, most of these models share a great many common structural features, and that 
differences between them fall into fairly well defined, general categories. The remainder of this section will focus on these similarities and differences in a general way, using examples drawn from individual models to illustrate important points.

\section{Mode 1 Structure}

In a world in which there is perfect certainty about future economic conditions and in which there are no institutional or technological constraints on the ability of consumers and producers to adjust instantaneously to changes in these conditions, input usage, the amount of acreage planted and harvested, production and consumption will all be determined simultaneously. These types of assumptions are typically employed within the framework of analytical models, such as those presented in Section 3.2 of this report, in order to develop the theoretical implications associated with a particular economic problem. In reality, of course, no such world exists. Producers and consumers are frequently forced to make a great many decisions based on inadequate information about current and future economic conditions, and, once made, it is of ten very difficult to adjust the outcome of these decisions consistent with new information that is acquired over time. Thus a world of perfect certainty and frictionless adjustments represents a highly inappropriate construct for designing models that are to be used in a positive framework to predict the actual behavior of consumers and producers in the real world.

However, it turns out that the existence of uncertainty, as well as technological and institutional rigidities within the agricultural sector, makes it easier rather than more difficult to model the underlying economic relationships that determine the amount of each commodity that is produced. This is because the existence of lags between the commitment of both variable and fixed inputs and the realization of output permits a recursive formulation within the equations that are used to determine output, up to the point of demand. A model is said to be recursive if its member equations can logically be arranged in terms of causes and events so that each dependent variable can be treated as a function of exogenous variables that have been treated as dependent at an earlier stage in the model. 
In order to better understand the recursive structure employed in these models, it is convenient to divide the agricultural production process into three stages that are more or less consistent with reality. These are: (1) the planning stage during which decisions are made regarding acreage planted, livestock purchases, commodity inventories, machinery purchases and changes in the stock of other kinds of physical assets; (2) the planting and production stage during which decisions are made regarding the optimal mix of variable inputs and fixed factors that are to be combined in production, consistent with the decisions made during the planting stage; and (3) the harvesting stage during which decisions are made regarding acreage harvested, livestock sales, and year-end stocks to be maintained. Some models (Ray 1971; Reynolds and Heady 1975) give very detailed treatment to decisions regarding the purchase of durable goods and the use of these assets along with variable inputs in stages (2) and (3), while others (Baumes and Meyers 1980; Collins 1980; Daniel and Steadman 1981) give very little consideration to the determination of input quantities.

Generally speaking, it is possible to model the supply response for a single crop, up to the quantity demanded, using three equations and a single identity. Traditionally, important decisions that are made in stage 1 are incorporated into an acreage response function, such that the number of acres planted can be expressed in the following form:

Planted acres $=f \quad\left\{\begin{array}{l}\text { (a) Expected price of planted crop } \\ \text { (b) Expected prices of competing crops } \\ \text { (c) Input prices } \\ \text { (d) Acreage planted in the previous period } \\ \text { (e) Government support prices } \\ \text { (f) Government acreage controls }\end{array}\right.$

Included in this process are: (a) the economic incentive to increase (decrease) the number of acres planted as the expected price of a crop increases (decreases); (b) the influence of the prices of competing crops based on the hypothesis that planted acreage will increase (decrease) as the expected prices of substitute crops decreases (increases); (c) the effect of input prices, such that increases in the relative price of an input that is 
used more intensively in one crop will cause the producer to devote more acreage to crops that use this input less intensively; (d) the effect of the previous years' planting decision due to lags associated with adjusting the actual number of acres planted to the desired number of acres; and (e) and (f) the influence of economic incentives and/or disincentives imposed through government programs offered to producers prior to planting.

Decisions that take place during Stage 2 are usually represented in terms of a yield equation. The average yield of a crop is sometimes treated as a exogenous input to the model, based on the premise that over short periods of time, farmers do not substantially alter input usage in response to changes in crop or input prices. In that case, variations in weather are treated as the most important determinant of crop yields. Where yield equations are explicitly included in the structure of the model, they are generally expressed in the following form:

$$
\text { Average yield }=f \quad\left\{\begin{array}{l}
\text { (a) Expected price of the planted crop } \\
\text { (b) Input prices } \\
\text { (c) Planted acreage } \\
\text { (d) Time trend } \\
\text { (e) Weather }
\end{array}\right.
$$

The expected price of the crop is included in this formulation to reflect the fact that, as the incentive to produce the crop increases, inputs will be used more intensively in order to raise yields, while changes in input prices affect the relative quantities of different inputs used in production. Planted acreage is included in the yield equations to reflect decreasing returns to scale in the use of that factor: other things being equal, the average yield of a crop will tend to decline as marginal lands are cultivated more intensively. The time trend in the model is inserted to reflect long-term improvements in yield due to technological change. Weather variables may or may not be given explicit treatment in the yield equations. When this is done, monthly precipitation and temperatures or monthly deviations from normal precipitation and temperatures are used to capture the variability in yields due to meteorological influences. Otherwise, this variability is assigned to the random error term in the yield equation. 
Stage 3 decisions, related to the supply responsiveness of the crop, are traditionally captured through an equation which relates acres harvested, recursively, to the number of acres planted, the historical ratio of harvested to planted acreage and the expected price at harvest, or

$$
\text { Harvested acres }=f \quad\left\{\begin{array}{l}
\text { (a) Acres planted } \\
\text { (b) Historical proportion of acres harvested } \\
\text { (c) Expected price at harvest }
\end{array}\right.
$$

The supply response model for each crop is then rounded out by a production identity in the form

$$
\text { PRODUCTION }=\text { Average Yield } \star \text { Acres Harvested }
$$

The supply response model determines total production via a recursive framework only up to the quantity demanded. Therefore, each commodity submodel must also include a model of demand. Demands are typically broken down into domestic and export commodity demands and private and government stock demands. There are substantial differences in the structure of demand equations used to model these different components for different agricultural commodities. Nonetheless, a fairly representative form that characterizes all of these demand components might be expressed as

$$
\begin{aligned}
& \text { Domestic } \\
& \text { Export } \\
& \text { Stock }
\end{aligned}
$$

Factors influencing these demands include: (a) the economic incentives for consumers and food processors to increase (decrease) the amount of the commodity demanded as its price decreases (increases); (b) the cross-price effect which changes in the prices of substitute or complementary goods will have upon the quantity demanded; (c) the effect of consumption during the 
previous period due to lags associated with adjusting the flow of consumption or stock demands to some desired level, and (d) the influence of exogenous variables, such as the effect of per capita income on the demand for food grains or the impact of the number of livestock on feed upon the demand for feed grains.

Supply and demand are usually treated independently up to the point at which the market clears. The market clearing identity is simply:

$$
S_{t}+Q_{t}^{D}=Q_{t}^{S}+S_{t-1}
$$

where $S_{t-1}$ and $S_{t}$ represent beginning-year and end-of-year stocks, respectively, $Q_{t}^{D}$ is the total of domestic and export demands and $Q_{t}^{S}$ stands for production duction plus exogenous imports. Current period commodity prices appear as independent variables which influence both stock and commodity demands through Equation (6.8). Supply, on the other hand, is predetermined by recursive forces up to the quantity demanded. By equating supply with demand through Equation (6.9), for each commodity, it is then possible to find the market clearing price, production and demand that are consistent with this equilibrium. Thus, the determination of equilibrium prices and quantities within the model is accomplished on a simultaneous basis, and these equilibrium values are subsequently treated as exogenous variables that influence supply and demand in the subsequent period.

\section{Lagged Adjustments}

Agricultural sector models usually employ two different types of lag structures in developing the recursive structure that is so highly characteristic of them. The first type of lag is associated with the stock adjustment model due to Nerlove (1958) and is used to reflect the effect of technical and institutional rigidities on the response of planted acreage. The stock adjustment model assumes that the desired level of acreage $\left(A_{t}^{*}\right)$ is dependent on expected prices $\left(P_{t}^{e}\right)$ and other variables $\left(Z_{t}\right)$, or

$$
A_{t}^{*}=\beta_{0}+\beta_{1} p_{t}^{e}+\beta_{2} Z_{t}+\varepsilon_{t}
$$


In any given period, however, the producer is not able to fully adjust the amount of acreage that is actually planted to the desired level due to technological and institutional constraints. An equivalent mathematical expression for this adjustment process is

$$
A_{t}-A_{t-1}=(1-\lambda)\left(A_{t}^{\star}-A_{t-1}\right) \quad 0<\lambda<1
$$

This equation specifies that the change in acreage will respond only partially to the difference between the desired amount of acreage and the acreage planted in the previous period, the rate of response being a function of the adjustment coefficient $\lambda$. Substituting for $A_{t}^{*}$ in Equation (6.10) and solving for the actual amount of acreage $p l a n t e d, A_{t}$, gives

$$
A_{t}=\beta_{0}(1-\lambda)+\beta_{1}(1-\lambda) P_{t}^{e}+\beta_{2}(1-\lambda) Z_{t}+\lambda A_{t-1}+(1-\lambda) \varepsilon_{t}
$$

The partial adjustment model explains the presence of the lagged acreage term $\left(A_{t-1}\right)$ in the acreage response equation shown in Equation (6.5). When the coefficient on that variable, $\lambda$, equals zero the adjustment is immediate; at the other extreme, when $\lambda=0, A_{t}$ never changes, i.e., $A_{t}=A_{t-1}$. For intermediate values of $\lambda$, the convergence of $A_{t}$ to $A_{t}^{*}$ is asymptotic. The rate of adjustment of $A_{t}$ to $A_{t}^{*}$ over time can be determined as follows: after $n$ periods, $1-\lambda^{n}$ of that adjustment is completed. Therefore, the number of periods required to achieve some proportion, $P$, of the desired adjustment can be calculated as

$$
n=\frac{\ln (1-P)}{\ln \lambda}
$$

Unfortunately, for empirical purposes, the expected price variable that appears in Equation (6.10) is not directly observable. This requires certain assumptions regarding the manner in which producers formulate their price expectations. 
Cagan (1956) describes a second, adaptive expectations model in which he hypothesized that the current expected price differs from the expected price in the previous period by an amount proportional to the previous forecast error, or

$$
P_{t}^{e}-P_{t-1}^{e}=\theta\left(P_{t}-P_{t-1}^{e}\right) \quad 0<\theta<1
$$

The adaptive expectations model can be substituted into Equation (6.11) to give

$$
A_{t}=\theta B_{0}+\theta B_{1} P_{t}^{e}+\theta B_{2} z_{t}+\theta A_{t-1}+\varepsilon_{t}-(1-\theta) \varepsilon_{t-1}
$$

This equation is virtually identical to the reduced expression for the stock adjustment model shown in Equation (6.12), except that the errors in $t$ and $t-1$ are serially correlated. In addition the acreage response equation still contains an argument in the unobservable variable $P_{t}^{e}$. However, Equation (6.14) can also be written as the solution to a first-order difference equation, or

$$
p_{t}^{e}=\theta \sum_{i=0}^{\infty}(1-\theta)^{i} P_{t-1}
$$

One can then select different values for $\theta$ and construct different price series for $P_{t}^{e}$ using Equation (6.16). Each of these series can be used, one at a $t$ ime, to estimate the parameters of Equation (6.10) until the best fit is obtained for that mode1, using ordinary least squares (OLS) methods. The resulting parameter estimates for that equation will then be consistent with conditional maximum likelihood estimates.

\section{Inclusion of Risk}

Including the effect of risk into agricultural sector models can be extremely difficult from a methodological standpoint. Hence, the treatment here is fairly general. More detailed exposition of the methodological problems encountered in this area can be found in Behrman 1968; Just 1974, 
1975. The importance of including risk in agricultural sector models relates directly to the impact of climate variability on crop yields: the greater the climate variability the more dramatic are the annual fluctuations in the price of a commodity about its long-term trend. Other things being equal, risk averse producers will select crops that are less sensitive, from a physiological standpoint, to climatic extremes in order to reduce the variability in their returns, even if it means sacrificing some amount of expected gain for reduced risk.

There are at least three different ways to include the effect of risk on production into the acreage response functions of an agricultural sector model. The simplest approach is based on the assumption that the influence of risk on planting decisions occurs primarily through the deviation of commodity prices in the previous period from moving average prices. Using this approach, the risk variable that is included in an acreage response equation can be expressed as

$$
r_{t}=\left[P_{t-1}-\frac{1}{N} \sum_{t=1}^{N} P_{t-2}\right]^{2}
$$

where $r_{t}$ is the risk variable associated with a particular crop, $N=t-2$ is the number of years used to compute the lagged moving average price, and where the deviation in the lagged price from the moving average is squared to reflect the responsiveness of risk due to large price deviations about the moving average price. The advantage of this approach over more complicated methods is that Equation (6.17) itse If contains no unknown parameters, and once $r_{t}$ is calculated it can be inserted directly into the appropriate acreage response equation and the parameter estimate for $r_{t}$ can be obtained by conventional OLS methods.

The second approach is only slightly more complicated, and is based on the assumption that the effect of risk on the number of acres planted is a weighted average of the squared deviations between actual and expected crop prices. This modified adaptive expectations model can be expressed using an expanded version of Equation (6.10) as 


$$
A_{t}^{*}=\beta_{0}+\beta_{1} p_{t}^{e}+\beta_{2} Z_{t}+\beta_{3} r_{t}+\varepsilon_{t}
$$

where the risk variable is defined as

$$
r_{t}=\sum_{i=1}^{\infty} \alpha_{i}\left(p_{t-i}-p_{t-i}^{e}\right)^{2}
$$

Unlike the previous method $r_{t}$ cannot be calculated from price data directly because of the presence of the $\alpha_{i}$ parameters in Equation (6.19). Therefore maximum likelihood search techniques are required to obtain an estimate of $r_{t}$ that is consistent with minimizing the sum of the residual errors in Equation (6.18) by means of conventional OLS methods. This procedure is similar to the one described for the estimation of the adaptive expectations model shown in Equation (6.16).

The final method involves applying the adaptive expectations model directly to risk in its complete form. Using this approach, the price risk variable can be represented by the following equation

$$
r_{t}^{e}=r_{t-1}^{e}+\phi\left[\left(p_{t}-p_{t-1}^{e}\right)^{2}-r_{t-1}^{e}\right]
$$

where $\theta$ is a weight on the squared deviations between actual and expected price and $r_{t}^{e}$ is the variance of the subjective distribution of prices in period $t+1$ as perceived in period $t$. The solution to Equation (6.20) is

$$
r_{t}^{e}=\theta \sum_{i=0}^{\infty}(1-\phi)^{i}\left(P_{t-i}-P_{t-i-1}^{e}\right)^{2}
$$

which looks very much like Equation (3.19), except that risk is now related to the squared deviations between actual and expected prices by a set of geometric weights. Notice, however, that the expected crop price $\left(P_{t}^{e}\right)$ appears in both of these formulations, as well as in the acreage response model shown in Equation 
(6.18). By using either of these two methods in conjunction with the adaptive expectations model, both $P_{t}^{e}$ and $r_{t}$ can be eliminated from Equation (6.18) and $p$ lanted acreage can then be expressed as a function of $z_{t}$, lagged price variables and the adjustment parameters that appear in both the price and risk adaptive expectations model. The reduced model is extremely complicated and will not be presented here. Estimating the parameters of such a model requires the application of fairly sophisticated maximum likelihood techniques (Just 1974). The advantage of this approach to the formulation of risk is that it incorporates the effect of risk directly into the formulation of expected prices, using a single consistent theoretical model.

\section{Inclusion of Meteorological Variables in Yield Equations}

All of the different types of models examined for showing the effect of $\mathrm{CO}_{2}$-induced environmental changes on crop yields pay very little attention to the interaction between economic and environmental conditions in the production of commercial crops. The effect of meteorological conditions on crop yields can be modeled in one of two ways. First of all, it is possible to omit meteorological variables entirely from the yield function and treat the impact of changes in weather on yield through the error term of the model. For example, a simple yield equation that is fairly widely used in the literature can be written as

$$
Y_{t}=\alpha_{0}+\alpha_{1} t+\varepsilon_{t}
$$

In this mode 1 , the parameter $\alpha_{1}$ shows the rate of change in average crop yields over time due to changes in technology. In any given period, average yield is equal to $\alpha_{0}+\alpha_{1} t$. Deviations in yield about this average are reflected in the variance of the error term $\varepsilon_{t}$. If $\alpha_{1}$ is assumed to be constant over time, then using additional information about future climate changes, the effect of these changes can be simulated by adjusting the value of $\alpha_{0}$ to reflect the impact of $\mathrm{CO}_{2}$-induced environmental changes on average crop yield and by changing the variance of $\varepsilon_{t}$ to reflect shifts in climate variability. 
A much more direct method involves taking into account the effect of changes in the values of meteorological variables explicitly within the framework of the crop yield model. Notice, that expanding Equation (6.22) to include meteorological variables results in a simple Thompson-type crop yield model, as shown previously in Section 5.2.2 of this report by Equation (5.10). By making $\alpha_{0}$ in Equation (6.22) depend on a vector of economic variables, including expected crop and input prices, $p_{t}^{e}$ and $p_{t}=\left(p_{1}, \ldots, p_{n}\right)$, respectively, planted acreage, $A_{t}$, and a vector of meteorological variables $W=\left(W_{1}, \ldots, W_{m}\right)$, Equation (6.22) can be rewritten in a more general form as

$$
Y_{t}=f\left(P_{t}^{e}, p_{t}, A_{t}, W_{t}, t\right)+\varepsilon_{t}
$$

The parameters of this revised yield equation can then be estimated from time series observations on the economic and meteorological variables (Weiss 1979).

At least three points need to be made in connection with this approach. The first of these is that the structure of Equation (6.23) will determine whether the effect of weather on crop yields is or is not independent of the effects of economic forces. This is an empirical and not a theoretical issue. Second, estimating the above equation employing national time series data will require constructing weather indexes from disaggregated regional data in order to avoid the "smoothing" effects that occur when using national data to obtain the annual values of relevant meteorological variables. Aggregate weather indexes have been developed by Stallings (1961) and Doll (1957); however, little consideration has been given to the nature of the aggregation bias that is introduced by the use of these indexes. Any bias that does exist--and it is likely to be substantial given the simplicity of these formulations--can be reduced by using Equation (6.23) in the framework of a regional agricultural sector model. Finally, because the parameters of Equation (6.23) are estimated using the same general statistical approach that is employed to estimate the parameters of statistical crop yield models, forecasting yields well outside the range of variability that is present in 
the values of the observed weather variables will generally increase the errors associated with the forecast. In statistical crop yield models this problem can be avoided to some extent by using data sets that already contain a substantial amount of variability. This is not possible in the framework of regional agricultural sector models, since the parameters of the yield equations in these models are meant to reflect regional conditions. otherwise, there is no justification for constructing regional models in the first place.

\section{Evaluation}

Application of the evaluation criteria developed in Section 6.1 of this chapter suggests the following set of conclusions regarding the use of econometric agricultural sector models to evaluate the direct economic impacts due to increases in the ambient concentration of $\mathrm{CO}_{2}$.

Dynamic Features. All of the econometric agricultural sector models examined in the course of this research incorporate supply models that are explicitly recursive through the use of stock adjustment and/or adaptive expectations models in determining the acreage response of individual crops as we 11 as changes in the numbers of livestock. It should be pointed out, however, that because these models are estimated using quarterly or annual data, the adjustment response that is captured within these models is largely due to short-term rigidities in the economic system. Long-run adjustments that may require 10 or 20 years to complete are not captured very we 11 by models employing quarterly or annual lags. Run over very long periods of time, it is extremely likely that these models will underestimate the ability of the agricultural economy to adjust favorably to gradual changes in the ambient concentration of $\mathrm{CO}_{2}$. This feature probably represents the most important single methodological shortcoming associated with the use of these models in any dynamic research framework. USDA economists have developed the NIRAP model specifically for long-term forecasting purposes. Th is model has recently been used to make projections of the impact of increased alcohol and sunflower production, respectively, in the agricultural sector to the year 2000 (Webb 1981; Watt 1981). However, the NIRAP model is poorly documented 
and it is not clear how the model can be manipulated to capture the impact of long-run adjustment mechanisms, apart from the effect of technological change.

Stochastic Capabilities. Only one model reviewed in the course of this research, POLYSIM (Ray and Richardson 1978), contains a stochastic simulator already built into its structure. Unfortunately, POLYSIM does not represent a viable alternative for examining the direct economic impacts of $\mathrm{CO}_{2}$-induced yield changes in the agricultural sector because shifts in supply and demand are calculated within the model using pre-determined constants to represent the price elasticities of the supply and demand for agricultural commodities. Both economic theory and a large body of empirical research demonstrate that these elasticities are not constant in most cases. In most of the remaining models reviewed these elasticities are determined endogenously within the model, which is as it should be.

In spite of this problem, treating crop yields in a stochastic framework is not methodologically complex. Yield equations, when they are present in a model, are always estimated with error, and the most important component in the variance of these errors is usually attributed to climate variability. Assuming no changes in climate variability, the influence of climate variability on equilibrium commodity prices and output can be simulated by drawing random normal deviates based on the distribution of the error terms on the crop yield equations in the model. Introducing changes in climate variability is only slightly more complicated, requiring that the distribution of these errors be changed over time, consistent with the information contained in individual climate-yield scenarios. The basic sampling process is the same. In either case, examination of the covariance structure of yields in the same region may make it possible to reduce the number of random deviates that must be drawn in each simulation, if the yields for some crops are correlated. Despite this possibility, the most serious drawback to the use of stochastic simulation will be the large number of simulation runs that must be performed to make accurate statements about the variance of the final forecast error for impact-related variables. 


\section{Regional and Commodity Disaggregation}

Surprisingly, only one model, AGSIM (Collins 1980), was uncovered that was built from the regional up to the national level. The Chase Econometrics model (Daniel and Steadman 1981) has a regional component; however, production in each region is determined largely based on its historical share of U.S. production, a top-down approach that does not include regional submodels for comodity supplies. AGSIM contains 13 crop regions, each of which contains a supply submodel. Demand is treated on a national basis so that regional supplies are marketed according to national prices. Crops included in the model are: (1) small grains which is an aggregate composed of wheat, barley and oats, (2) grain sorghum, (3) cotton, (4) soybeans, and (5) corn. In all, the model contains 51 region-crop combinations. A national livestock sector has recently been added, although it is not documented, which includes cattle and hogs. Thus, the number of commodities included the AGSIM is considerably less than the number included in most national models (for exmple, the Chase Econometrics model contains 14 crops and 6 different livestock categories). However, the number of region-crop combinations far exceeds the number of supply submodels in any of the other agricultural sector models reviewed for this report.

Linkages to the Rest of the World. National and regional models of the U.S. agricultural sector are inappropriate tools for analyzing the direct economic impacts that may occur within the U.S. as a result of the differential effects of $\mathrm{CO}_{2}$-induced environmental changes on agricultural production on a world-wide basis. USDA currently has one model, the world grains, oilseed and livestock (GOL) model (USDA 1978) which could be used in this framework. The GOL model is a multi-country and multi-region, world agricultural model consisting of supply, demand, and trade components for 12 comodities and 28 world regions. In its structure, the GOL model resembles a multi-regional agricultural sector model of the U.S., except that it contains explicit price linkages between world regions, as well as world equilibrium equations (i.e., supply = demand) for each comodity. The model was built primarily for making long-range projections of the world food supply-demand 
balance under alternative scenarios, including the impacts of climate change (USDA 1978). The GOL model is currently being updated to include nonlinear supply and demand equations.

Data Needs and Availability. Given the theoretical problems associated with predicting the characteristics and scope of long-term technological changes that will occur over the next 50 to 100 years in the agricultural sector, as well as the tremendous uncertainty that exists about the nature of $\mathrm{CO}_{2}$-induced yield changes, the benefits of constructing new agricultural sector models more suited to analyzing the $\mathrm{CO}_{2}$ problem are probably not very great, at this time. The one possible exception to this statement, which would involve additional data gathering and model estimation, lies in the area of incorporating longer temporal lags within the dynamic structure of this type of mode1. From a methodological standpoint, this would not involve any structural modifications to the stock adjustment and adaptive expectations models that are incorporated within the supply response equations for individual commodities. What is required is a much longer set of historical data series for the variables that appear in the supply response and demand equations of these models. This would then make it possible to lengthen the interval between $t$ and $t-1$ incorporated within the lag structure of the model from a single quarter or year to 5 or 10 years. The model parameters that are estimated using this data would then reflect the historical ability of producers and consumers to make long-run adjustments to changes in economic conditions.

The major drawback to this approach is that the scope of agricultural data coverage narrows as one goes back in time. Generally speaking, pre-1948 data are limited almost entirely to those which can be obtained from published census sources. The first agricultural census was taken in 1840; however data prior to c. 1900-1910 are of relatively poor quality. Prior to 1920, the agricultural census was taken every 10 years; thereafter, data were collected every 5 years, although the interval between agricultural census varies somewhat due to adjustments made in reporting. This means that any model that is estimated during the period 1920 to 1980 will have, at most, 13 observations per variable. This "degrees of freedom" problem not only limits 
the number of explanatory variables that can be used in any one equation, but also the sophistication of the econometric methods that can be applied in order to obtain unbiased, consistent parameter estimates. Hence, the advantages that such a model might possess in terms of its ability to capture historical, long-run adjustment behavior may be overwhelmed by data limitations. However, the seriousness of this tradeoff cannot be assessed without additional research.

Another area in which this criteria must be applied relates to the potential advantages of re-estimating individual yield equations in these models to explicitly capture the effect of changes in the values of meteorological variables in crop yields. The chief advantages of doing this are that (1) it eliminates the need to estimate statistical crop yield models to uncover the nature of these relationships (but not those related to other types of $\mathrm{CO}_{2}$-induced environmental changes); and (2) the independence of meteorological effects from those due to technology on crop yields does not have to be assumed, a priori. A meteorological data base specifically suited for this type of estimation has already been constructed, and is operational within USDA (Weiss 1979). Moreover, this type of modification to existing models could be accomplished without major structural modifications to existing models. However, the expense would depend on the number of yield equations that would have to be re-estimated. For example, endogenizing the influence of meteorological conditions within the AGSIM model would require re-estimating 51 yield equations. Since the statistical fit of these equations in AGSIM is generally not as good as for other model components, it is likely that this additional work would improve the overall accuracy of the mode 1 .

Statistical Accuracy and Model Stability. There are least three different areas in which the statistical accuracy of positive models creates fairly serious problems in using these models to evaluate long-term economic impacts. These are:

1. model parameters do not reflect long-term historical adjustments to changes in economic conditions; 
2. changes in future technology cannot be predicted; and

3. even in short-term forecasting situations, the accuracy of these models will decrease as the size of the exogenous shocks that are simulated in the economy grow in magnitude.

A possible remedy to (1) has already been suggested. The second problem can be addressed to some degree by manipulating those parameters within the model that are intended to capture the effect of technological change in a manner consistent with assumptions that are made about the scope and rates of future technological change. The third problem is an inherent limitation in all positive models, whether they are designed to predict crop yields or economic impacts. The only possible remedy to this problem is to increase the number of data points that are used to estimate model parameters, on the presumption that this will increase the variability that is present within individual data series for each variable.

None of these models examined in the course of this research were actually tested for stability. The results of any such stability tests that were conducted for these models did not appear in any of the documentation received. It was learned, however, that very recent sensitivity tests performed on the AGSIM model revealed that the model was stable over long periods of time--one hundred years--even when exposed to fairly substantial exogenous shocks. Any model that is ultimately selected to evaluate the economic impacts due to future increases in the ambient concentration of $\mathrm{CO}_{2}$ must be tested for stability prior to its use.

\subsubsection{Normative Models of the U.S. Agricultural Sector}

The U.S. agricultural sector is characterized by substantial heterogeneity with respect to differences in climate, soil types, farm practices and farm structure. Due to these variations, models designed to evaluate the economic impacts of exogenous shocks to the agricultural sector should reflect the highly regionalized character of the U.S. agricultural economy. Mathematical programing models, as a rule, are far more capable of reflecting these differences than are econometric models. The underlying reason for this relates to the ability of these models to characterize the 
behavior of production systems in concrete, physical terms. The equations that are contained within the supply response components of agricultural sector models are highly abstract representations of behavior, based on relationships derived largely from economic theory. Normative models, on the other hand, characterize technological relationships based on the actual flow of resources through the different stages of production systems. As such, the technological parameters that are used in programing models represent constants that describe the rate at which these resources are utilized in different processes, whereas the meaning of the parameters that appear in econometric models are abstract. Because of this major difference, normative models can be built to reflect the "representative technology" of the farm unit at virtually any level of regional specificity, using a wide variety of primary and secondary data sources. This does not entirely eliminate the aggregation problem in normative models (Day 1963) but it does make possible to reduce the amount of heterogeneity present within the agricultural sector to a much greater extent than is possible using the econometric approach.

Substantially increasing the number of regional units in mathematical programming models of the agricultural sector is very costly and time-consuming. The constraint matrix required to characterize the resource and commodity flows for a single crop in a model with 100 regions can easily exceed a dimensionality of $1000 \times 1000$ (Patton 1980)! Furthermore, because it is not methodologically difficult (although it is expensive) to expand programing models to include more production activities, transportation flows, between regions and additional resources, an initial advantage in this field of agricultural sector modeling can be quickly translated into a monopoly. This is essentially what has happened in this area. At present, there is only one large mathematical programing model of the agricultural sector in existence. This is the model currently housed at the center for Agricultural and Rural Development (CARD) at Iowa State University. A somewhat smaller model, developed at the University of Illinois (Taylor et al. 1976), has since been destroyed. Because the conceptual approach emtodied in these two models is somewhat different, the following discussion of model structure will focus on the importance of the alternatives offered by them. 


\section{Model Structure}

Most of the normative models that will be examined in this section of the report are basically linear programming models: both the objective function and the characterization of technology in the constraint matrix can be described by linear equations. Linear programming models were originally used in the agricultural sector as planning models, to determine optimal cropping patterns and the impact of resource limitations at the farm or small region level (Heady and Candler 1964; Dean and Carter 1961; Shumway et al. 1970). More recently, large linear programming models have been used extensively by agricultural economists to simulate the impact of rising energy prices, alternative water quality and supply alternatives, and a wide variety of farm programs on the agricultural sector (Adams 1976; Droskin et al. 1978; Wade and Heady 1974; Meister and Nicol 1975). However, as McCarl and Spreen have noted (1980) these large-scale linear programing models have included the restrictive assumption, carried over from farm and small region models, of fixed commodity prices or output. As such, the solutions they provide are inconsistent with the simultaneous determination of supply and demand under competitive market conditions.

The models that are discussed in this section of the report represent a second generation of normative, agricultural sector models. These models retain many of the basic characteristics of traditional, price-exogenous linear programming models; however, they also contain features which allow them to generate normative solutions that are consistent with a competitive equilibrium. For convenience, differences between these models can be broken down according to whether the objective function of the model is specified in terms of (a) criteria that are consistent with the economic behavior of individual firms (i.e., cost minimization or profit maximization) or (b) aggregate welfare criteria, namely, the sum of producer and consumer surplus.

Models Based on Firm-Level Objectives

There are two basic types of models that fall under this heading:

(1) cost minimization models which use a simulated tatonnement process to 
achieve equilibrium price and quantity solutions between parametrically generated supply curves from within the model and demand curves that are external to the mode1; and (2) recursive programming models which use an econometric model of the agricultural sector to revise the commodity price vectors and acreage flexibility constraints within a profit maximization model. Only two models--one representing each of the approaches described above--were uncovered during the course of this research. Both are based on the CARD linear programming model (CARD LP) which is documented in Meister and Nicol (1975), Nicol and Heady (1976) and English et al. (1981a). The tatonnement features of the first model are described in English et al. (1981b), while the recursive model is documented in Langley et al. (1981). For the purposes of brevity these two models will be referred to as the CT (CARD tatonnement) and CR (CARD recursive) models.

The cost minimization component of the CT model can be described in matrix notation as

$$
\begin{aligned}
& \text { Minimize } C^{\prime} X \\
& \text { subject to } \\
& A X \leq R \\
& Y X \geq D \\
& X \geq 0
\end{aligned}
$$

where

$$
\begin{aligned}
C= & n \times 1 \text { vector of variable costs due to a one unit increase in the } j \text { th } \\
& \text { activity } \\
x= & n \times 1 \text { vector of activity levels, which includes } 1, \ldots, k \text { production } \\
& \text { activities and } k+1, \ldots, n \text { other activities }(j=1, \ldots, k, k+1, \\
& \ldots, n) \\
A= & m \times n \text { matrix of technology coefficients expressing the amount of the } \\
& \text { ith resource required per unit increase in the } j \text { th activity }(i=1, \\
& \ldots, m)
\end{aligned}
$$


$R=m \times 1$ vector of resources

$Y=k \times k$ matrix of output coefficients expressing the physical output associated with a unit increase in jth activity, $1, \ldots, k$ inclusive

$D=k \times 1$ vector of output demands

The solution to this problem is characterized by the following outputs:

- An optimal output vector $x^{\star}=\left(x_{1}, \ldots, x_{n}^{\star}\right)$ of nonnegative activity levels.

- A vector of opportunity costs, $\Psi=\left(\Psi_{1}, \ldots, \Psi_{n}\right)$, such that if $x_{j}>0$, then $\Psi_{j}>0$ and if $x_{j}=0$, then $\Psi_{j}=0$; each opportunity cost that appears in the optimal solution represents the marginal cost to the U.S. agricultural sector as a whole of employing the jth activity.

- A vector of shadow prices $\lambda=\left(\lambda_{1}, \ldots, \lambda_{m}\right)$, associated with each of the rows in the $A$ matrix, such that if $R_{j}$ is fully utilized in production, then $\lambda_{j}>0$, otherwise $\lambda_{j}=0$; each of these shadow prices reflects the increase in marginal revenue that would accrue to the agricultural sector (assuming exogenous prices) if one more unit of the ith resource were made available.

- A vector of positively valued shadow prices, $\phi=\left(\phi_{1}, \ldots, \phi_{k}\right)$, associated with each of the constraints in the $Y$ matrix; each of these shadow prices represents the marginal cost to the system due to a one unit increase in the demand for the kth commodity.

The tatonnement process used in the $\mathrm{CT}$ model to arrive at a competitive equilibrium solution works as follows. The cost minimization model is first solved for an arbitrary vector of commodity demands, $D_{t}$, resulting in a corresponding vector of commodity output, $Q_{t}^{S}$, and a vector of shadow prices, $\phi_{t}$, which represent supply prices. These supply prices are then inserted into a system of commodity demand equations that are external to the cost minimization model (but internal to the CT model) to solve for the vector of demands, $Q_{t}^{D}$, that is consistent with the supply price vector. Next a weighted average of $Q_{t}^{D}$ and $Q_{t}^{S}$ is used to determine a new value, $D_{t+1}$, for constraining the cost 
minimization model. The cost minimization model is again solved using $D_{t+1}$ as the new demand constraint. This process is then repeated unt $i 1$ the values of $Q_{t}^{D}$ and $Q_{t}^{S}$ converge to an appropriately small tolerance level. The resulting solution satisfies the general equiribrium condition that the final equilibrium price, $\phi^{\star}$, must eliminate excess demands, such that $Q_{t}^{D}-Q_{t}^{S} \approx 0$. Simulations performed, using the tatonnement process described above, thus far have shown that this method is remarkably stable: convergence is usually achieved within 2 to 4 iterations, for a tolerance level of $1 \%$, or

$$
\frac{\left(Q_{t}^{D}-Q_{t}^{S}\right)}{Q_{t}^{D}} \leq 0.01
$$

The CARD cost minimization model that represents the heart of the CT system is a very large interregional linear programming model. Included in the model are 105 producing areas (PAs) and 28 market regions (MRs). Commodities are produced in each of the 105 regions, using three basic resources--the land base, water and nitrogen fertilizer. Commodity demands, which represent the driving variables in the model, are determined in the 28 MRs. In addition, there are 176 transportation activities defined to simulate transportation routes between MRs via barge, rail and truck. As such, interregional commodity flows that occur within the model play an important role in determining the spatial price-quantity equilibrium that is ultimately ach ieved.

Production activities in the model include crop and livestock production, water availability, nitrogen purchase, irrigation and land development, and water hay. Barley, corn grain and silage, cotton, legume, and nonlegume hay, oats, sorghum grain and silage, soybeans, wheat and summer fallow are produced with in the model through dryland and irrigated production practices. The livestock sector, which has recently been added and is not documented, includes beef on feed and nonfed beef, hogs and dairy products. The water sector, consists of four types of activities that permit the purchase of additional water, interbasin transfers, increased water use efficiency and the 
production of water hay. The nitrogen purchasing activities supply commercial nitrogen to the crop production sector. Additional land is made available through land development activities. The allocation of this land between dryland and irrigated production, as well as the rate at which existing dryland acreage is converted into irrigated acreage (and vice-versa), then depends on the relative profitability of dryland versus irrigated agricultural production.

The CR model consists of four components: (1) the CARD linear programming model, (2) an econometric model of the U.S. agricultural sector, (3) a subroutine that uses the output of the econometric model to revise parameters within the linear programming model, and (4) a subroutine that adjusts the final equilibrium price vector for agricultural commodities based on a comparison of the two models. The CR model uses an earlier and somewhat smaller version of the linear programing model that is employed in the CT system. Also, the objective function is expressed in terms of maximizing the net returns to production, rather than cost minimization. The econometric model used in the CR system is a dynamic simulation model of the U.S. agricultural sector which contains national livestock, wheat, feed grain, soybean and cotton submodels (Ray 1971; Reynolds and Heady 1975). Within each submodel, a set of pre-input equations estimates acreage planted, commodity stocks, machinery purchases and other physical assets necessary for planting crops and raising livestock. The input section of each submodel estimates the values of production inputs consistent with decisions reflected in the pre-input section. A set of output equations then determines livestock and crop production, prices, inventory levels and income. Endogenous variables determined in the output section of each submodel in period $t$ are then used as explanatory variables in the pre-input equations in $t+1$. Thus, the model is recursive both with in the crop year and between crop years.

Figure 6.2 illustrates how the four components of the CR model interact on a dynamic basis. Initially, the econometric model is used to simulate the behavior of the agricultural sector for a single period, given a pre-specified set of initial conditions. This simulation is represented by $E_{t}$. Price, 


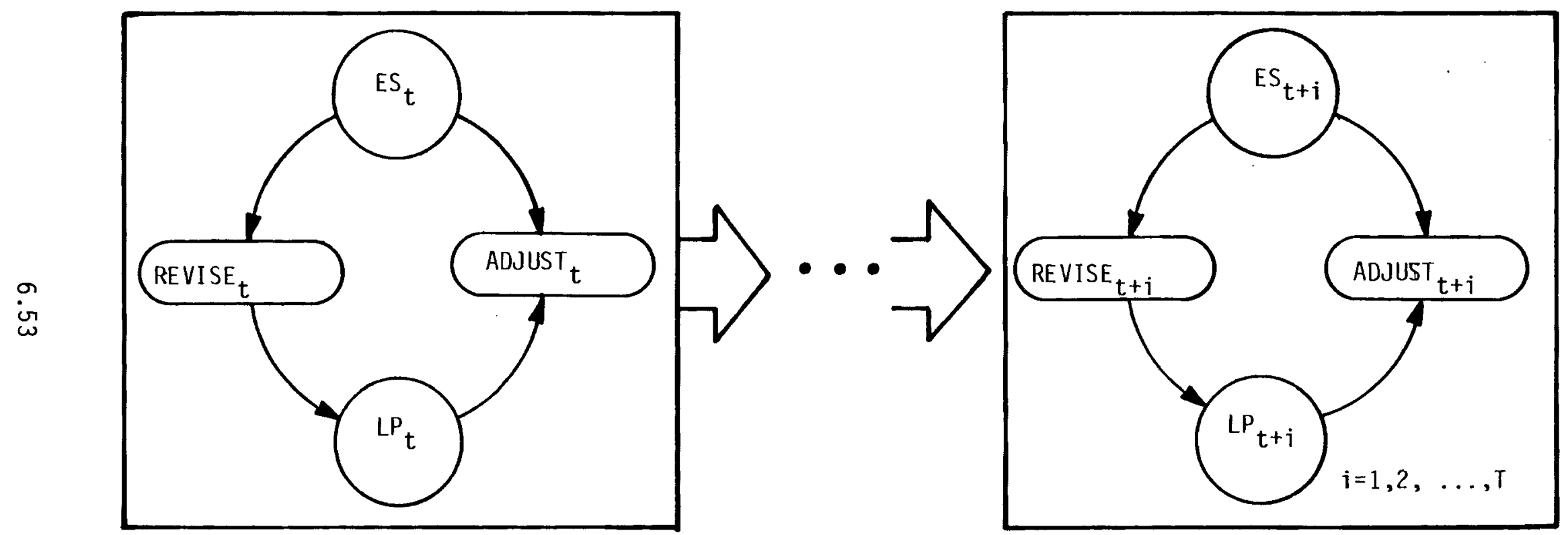

FIGURE 6.2. A Schematic Diagram of the CARD Recursive (CR) Model (Source: Langley et al. 1981) 
production and cost estimates from the econometric model are fed into the REVISE submodel. Price and cost information are used to revise the net price parameters for each of the crop and livestock activities in the linear programing model $\left(L P_{t}\right)$. Production and price information are used simultaneously to constrain the production of individual commodities in the LP model within specific upper and lower bounds. At this point in the process, price and production information are also fed from the ES model to the ADJUST subroutine. The LP model is then run, and the optimal vector of commodity outputs obtained from the solution (consistent with earlier revisions) is fed into the ADJUST subroutine. This subroutine compares the two estimates of commodity production and if the estimates are the same no adjustment is made. However, if the two estimates are different, the following adjustment is made

$$
P_{i t}^{\star}=P_{i t}-\alpha_{i}\left(Q L P_{i t}-Q E S_{i t}\right)
$$

where $P_{i t}$ is the aggregate price estimate for the ith commodity in period $t$, QLP $_{\text {it }}$ and $Q E S_{i t}$ represent production estimates from the LP and ES models respectively and $\alpha_{i}$ is an adjustment parameters obtained from the relevant commodity price equation in the ES model.

The price adjustment process that is indicated by Equation (6.26) permits the CR model to simulate adaptive behavior on a dynamic basis. Bear in mind that $Q_{\text {LP }}$ it is a normative estimate of potential production (consistent with revised prices and costs), while QES it is a positive estimate. Therefore, when optimal production is greater than actual production, this implies that the agricultural sector is not operating at its full potential and that excess capacity will lead to downward pressure on prices. Under these conditions, resurces will be bid out of the agricultural sector and production will cut back until such time as stocks are sufficiently eroded and/or increased demand leads to high enough prices to expand production. On the other hand, when QLP $_{i t}<Q_{i t}$, this implies that the agricultural sector is operating at a close to full capacity and that increased demand for scarce resources will put upward pressure on commodity prices. Under these conditions, resources will 
be bid away from other sectors and production will increase in response to higher expected commodity prices, leading to an increase in agricultural capacity.

Thus, the CR model is substantially different from the CT system in that is is dynamic, whereas the latter is static, reflecting instantaneous adjustment to a normative competitive equilibrium. However, the adjustment mechanisms built into the CT model are also different than those contained in econometric models in that it combines the anticipatory nature of an explicit planning model with the recursive aspects of economic behavior that are embedded in the structure of an econometric model.

Models Based on Welfare Objectives

Both the CT and CR models require auxiliary models and/or subroutines in order to achieve competitive equilibrium solutions. A second alternative involves incorporating the objective of maximizing the sum of producer and consumer surplus into the objective function of the cost minimization problem through separable programing methods (Duloy and Norton 1975). Recall from Section 3.3.2 that this measure of welfare represents the difference between the total willingness of consumers to pay for a commodity and the cost of producing that commodity. This concept is illustrated again for clarity in Figure 6.3 for the case of a single good. The total willingness of consumers to pay for $Q^{\star}$ is represented in the area $\mathrm{DMQ}^{\star} 0$. The total cost of producing $Q^{\star}$ is $O M Q^{\star}$. The difference between these two areas or $D M O$, is the sum of producer surplus ( $\left.P^{\star} M O\right)$ plus consumer surplus ( $\left.P^{\star} D M\right)$. Since the cost minimization problem shown in Equations (6.25) and (6.26) approximates total cost for producing $Q^{\star}$, changing the model to reflect this welfare objective requires an approximation for consumer willingness to pay.

This can be accomplished by linearizing the integral of the indirect demand function as shown in Figure 6.4. The top diagram is a representation of this integral, where

$$
f(Q)=\int_{0}^{Q^{\star}} P(Q) d Q
$$




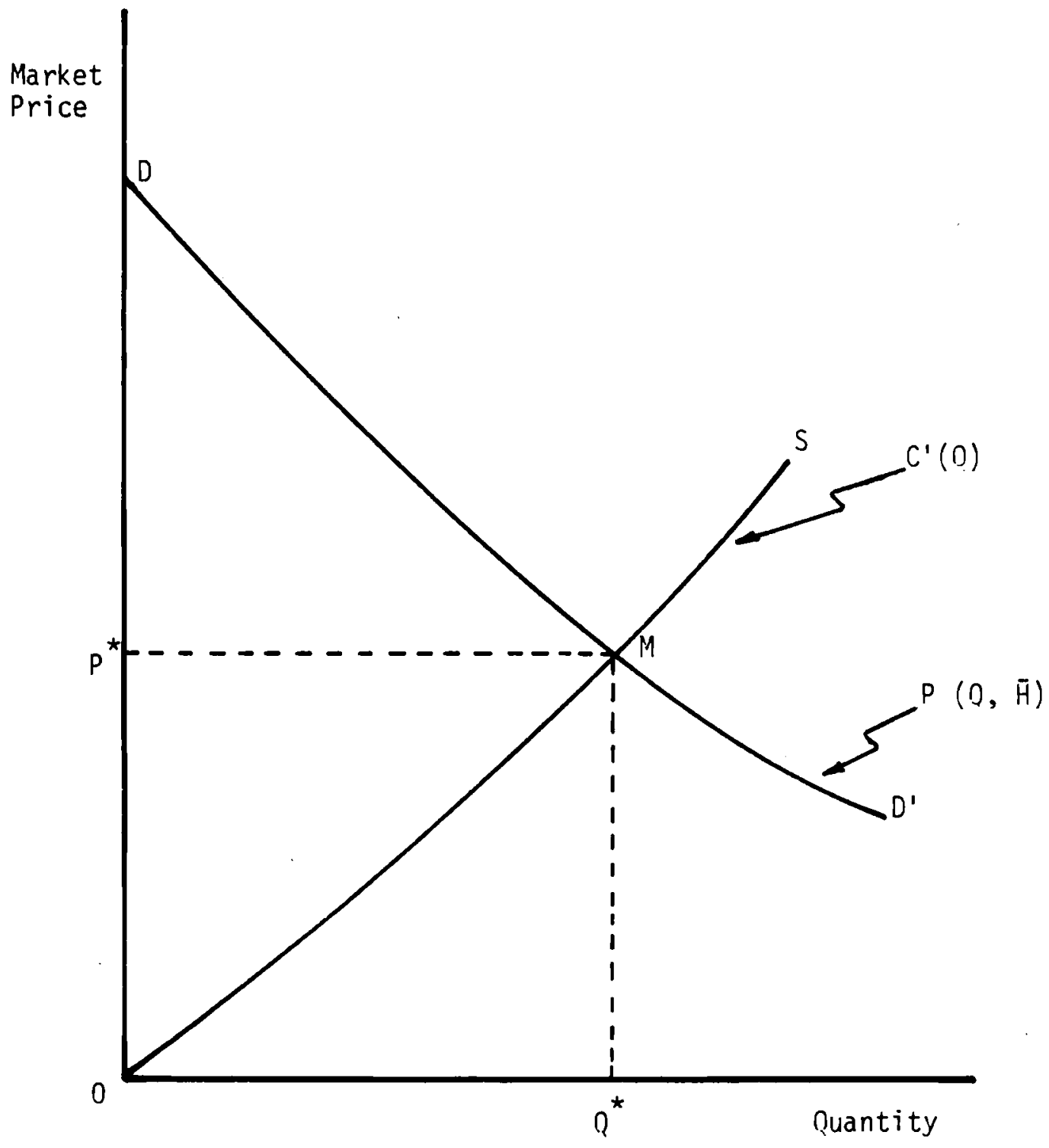

FIGURE 6.3. Illustration of Producer and Consumer Surplus 


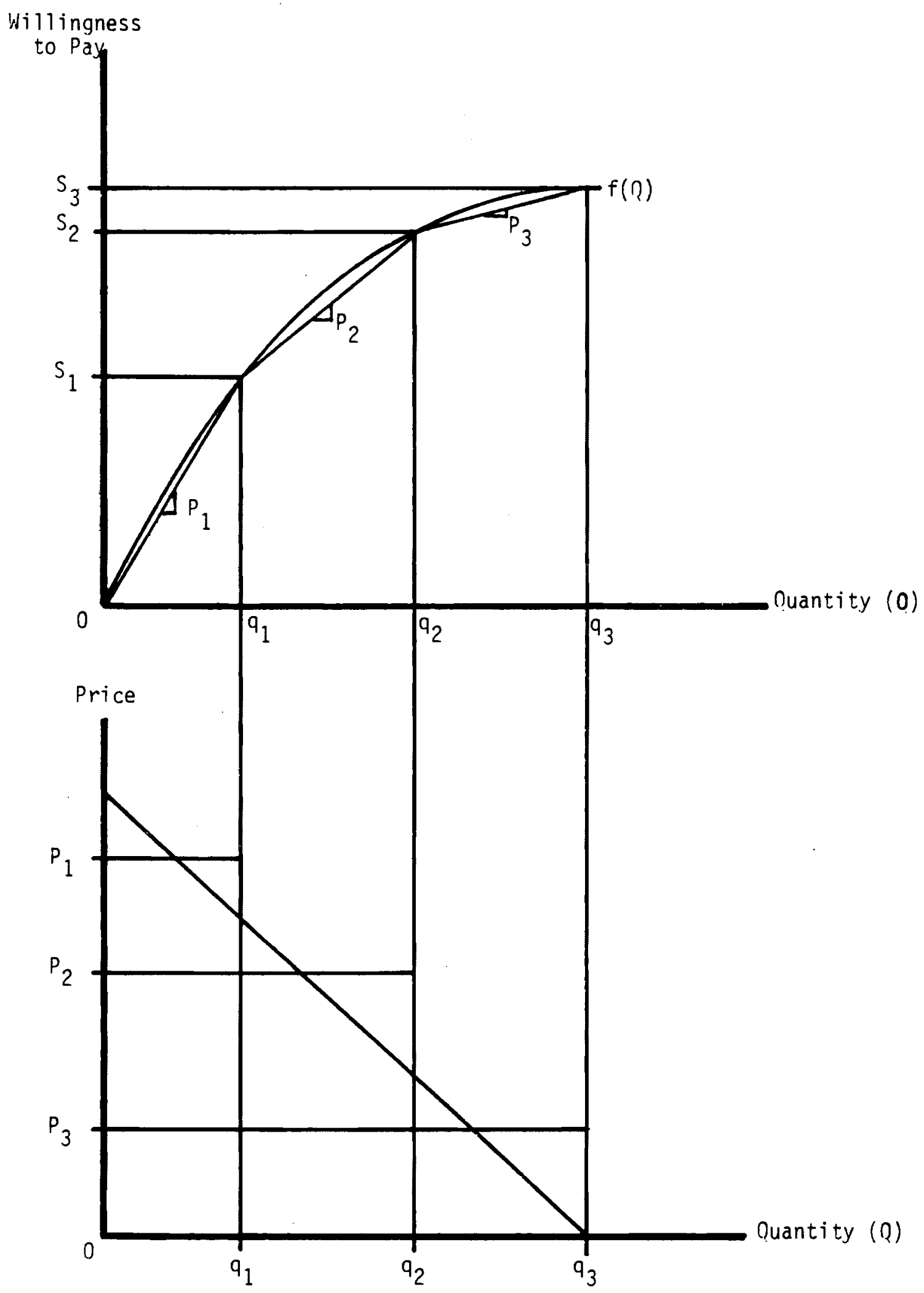

FIGURE 6.4. Linearization of Willingness to Pay Functions

$$
6.57
$$


equals the area under the indirect demand function $P(Q)$ shown directly below it. The first step in approximating the area involves dividing $f(Q)$ into a series of chords, $O A, O B$ and $O C$, and computing the corresponding values of consumer willingness to pay at each endpoint, or $0, s_{1}, s_{2}$, and $s_{3}$. The more chords that are constructed, the more accurate the approximation of $f(Q)$. The resulting approximation can be expressed as

$$
f(Q) \approx . O B_{0}+s_{1} B_{1}+s_{2} B_{2}+s_{3} B_{3}
$$

where the $B_{0}, \ldots, B_{3}$ terms represent variable weights which are required to be nonnegative and to sum to unity. One can then express the total quantity of $Q$ that is demanded in a similar type of form

$$
Q \approx O B_{1}+q_{1} B_{1}+q_{2} B_{2}+q_{3} B_{3}
$$

where $q_{1}, q_{2}$ and $q_{3}$ represent the value of $Q$ that coincides with $s_{1}$, $s_{2}$ and $s_{3}$, respectively.

To show how Equations $(6.27)$ and $(6.28)$ can be incorporated into the basic linear programming format to provide a competitive equilibrium solution, a simple example is provided. Assume that there are $i=1, \ldots, m$ fixed resources $\left(b_{i}\right)$ in the model $, j=1, \ldots, n$ production activities $\left(Q_{j}\right)$ each of which involves a different comodity, and that $h=0, \ldots, k$ weights $\left(B_{h}\right)$ are used to linearize $f(Q)$ for each of the $n$ commodities. Consistent with these assumptions, one can express the programing problem as

$$
\operatorname{Maximize} W=\sum_{j=1}^{n} \sum_{h=0}^{k} s_{j h} B_{j h}-\sum_{j=1}^{n} c_{j} x_{j}
$$

subject to

$$
\sum_{j=1}^{n} a_{i j} x_{j} \leq b_{i} \quad \text { for all } i
$$




$$
\begin{array}{ll}
-y_{j} x_{j}+Q_{j} \leq 0 & \text { for all } j \\
\sum_{h=0}^{k} a_{j k} B_{j h}-Q_{j} \leq 0 & \text { for all } j \\
\sum_{h=0}^{k} B_{j h} \leq 1 & \text { for all } j \\
\text { all } x_{j}, Q_{j}, B_{i h}>0 &
\end{array}
$$

If the original $f(Q)$ 's are convex, then

$$
\sum_{h=0}^{k} B_{j h}=1 \quad \text { for all } j
$$

and, for each of the approximations, no more than two of these weights will be positive and these will be adjacent. Thus, the approximate value of consumer willingness to pay for each commodity will lie somewhere on the chord in between these two weights, the exact position being a function of the two weights. The optimal price and quantity equilibrium for each comodity will be given in the solution by the shadow price in Equation (6.29c) and the optimal value $Q_{j}^{\star}$. Thus, prices and quantities are fully endogenized in this model, whereas as they are not in the conventional linear programming model.

This approach is used in Taylor et al.'s (1976) agricultural sector model and in Norton and Duloy's CHAC model of the Mexican agricultural sector (1973a; 1973b). This approach can also be incorporated into existing versions of the CARD linear programing model without any computational difficulties. The major drawback of separable programming is size. Each segment in $f(Q)$ requires the addition of one column to the objective function and the constraint matrix, while each additional commodity requires another two constraints. If each $f(Q)$ was modeled with 10 segments, this would add several thous and new rows to the CARD model, and 10 times as many columns! 
Hence, this approach was rejected by the authors of the CT model in favor of the tatonnement approach used in that system.

Evaluation

Application of the evaluation criteria developed in Section 6.1 of this chapter suggests the following set of conclusions regarding the use of linear programing based models of the agricultural sector to evaluate the direct economic impacts due to increases in the ambient concentration of $\mathrm{CO}_{2}$.

Dynamic Features. While linear programming models can be constructed to incorporate intertemporal objectives, the major difficulty with doing this is that the physical linkages between periods, through stock flows, must be characterized explicitly. Thus, the static constraint matrix must be defined for each period, joined together by carry-over stocks from the previous year and end-of-year stocks in the current period. Given the initial size of the CARD model, this type of modification is unthinkable. On the other hand, the CR model is explicitly dynamic due to the linkages that exist within it between the CARD model and a recursive econometric model. The accuracy of this model in making export forecasts has not been reported, al though simulations of the effects of alternative export-important and farm program alternatives show plausible results for short-term forecasts. The CR model appears to show some promise for the analysis of long-run adjustment behavior due to the fact that $P_{i t}$ in Equation (6.26) is determined by the interplay between the normative and positive estimates of commodity output. In that framework, arbitrarily increasing the value of the adjustment parameters in this equation would appear to be consistent with long rather than short-term adjustments.

Stochastic Capabilities. Conceptually, Monte Carlo simulation with a linear programming based model is no different than in other applications. The problem is simply one of cost. Each solution of the CT model costs from $\$ 1200$ to $\$ 1600$ depending upon the number of iterations required to achieve an equilibrium solution. The CR model costs from $\$ 100$ to $\$ 250$ for a three-year run, using a small CARD linear programming model. This can be contrasted with 
a cost of approximately $\$ 100$ to $\$ 250$ for a 100-year run on an early version of the AGSIM econometric model. In addition, none of the normative models examined for this report are structured to take into account the influence of the stochastic nature of climate on agricultural production due to risk averse behavior on the part of farmers. The authors of the CR model have proposed such a modification (Langley et al. 1981), but it has not been implemented.

Regional and Commodity Disaggregation. One of the real strengths of linear programming based models lies in the large number of regions, comodities, and alternative process activities that can be included. Particularly important in this regard is the ability of the CT model to incorporate land and water resources development, transfers of land between dryland and irrigated agricultural practices and interbasin transfers of water--all at the regional level. Since dramatic adjustments of this type are at least theoretically consistent with substantial changes in the magnitude and geographic distribution of crop yields, this is a very attractive feature, and one that is not within the range of any econometric model.

Linkages to the Rest of the Wor ld. Both the CT and CR models treat exports and imports as exogenous. If the impact of $\mathrm{CO}_{2}$-induced environmental changes in other nations on the U.S. agricultural sector is to be examined, this will require the development of spatial price allocation models (Takayama and Judge 1971). These models are formulated in terms of the following quadratic programing problem

$$
\text { Maximize } \begin{aligned}
W & =\sum_{i=1}^{m} \sum_{r=1}^{n}\left[\theta_{i r}-\frac{1}{2} \psi_{i r} Q_{i r}^{D}\right] Q_{i r}^{D} \\
& -\sum_{i=1}^{m} \sum_{r=1}^{n}\left[\alpha_{i r}+\frac{1}{2} \phi_{i r} Q_{i r}^{S}\right] Q_{i r}^{S} \\
& -\sum_{i=1}^{m} \sum_{r \neq k}^{n} \sum_{k}^{n} t_{i r k} x_{i r k}
\end{aligned}
$$


subject to

$$
\begin{aligned}
& \sum_{k=1}^{n} x_{i r k}-Q_{i r}^{S} \leq 0 \quad \text { for } a l l i \text { and } r \\
& -\sum_{r=1}^{n} x_{i r k}+Q_{i k}^{D} \leq 0 \quad \text { for } a l l i \text { and } k
\end{aligned}
$$

where $Q_{i r}^{D}$ and $Q_{i r}^{S}$ represent the demand and supply of commodity $i$ in each of the $r=1, \ldots, n$ trading regions, $t_{i r k}$ is the unit cost of transporting commodity $i$ from region $r$ to region $k$, and $x_{i r k}$ is the amount of the commodity $i$ traded from region $r$ to region $k$.

The objective function in Equation (6.30) is expressed in terms of the sum of producer and consumer surplus. The first two quadratic expressions represent the total willingness of consumers to pay for and the cost of all of the $m$ commodities in all of the $n$ regions. The third expression characterizes the costs of transportation. The two constraints included in the problem represent supply and demand trade balances, respectively. Thus, in Equation (6.31a) the supply of commodity $i$ in region $k$ will be equal to or greater than outshipments to other regions, while Equation (6.31b) requires that the consumption of commodity $i$ in region $r$ must be less than or equal to the amount shipped in from other regions. The optimal solution to this problem consists of optimal vectors for regional production, output and international commodity flows and shadow prices.

A number of models have been built around this framework (Simmons and Pomereda 1975; Cappi et al. 1978; Meister et al. 1978), some of which are currently used by USDA for policy purposes (USDA 1981). However, these models are usually restricted in scope to cover only a few commodities or a small number of regions. Thus an international model of the dimensions required to evaluate the effect of $\mathrm{CO}_{2}$ on the U.S. agricultural sector through foreign trade linkages would have to be built from the ground up. Even then, serious problems related to the empirical specification of the willingness to pay and 
cost functions would still exist (McCarl and Spreen 1980), as would the problem of incorporating the impact of $\mathrm{CO}_{2}$-induced yield changes into the supply functions of any such model.

Data Needs and Availability. Given the size of the effort required to build linear programming based models of the agricultural sector from the ground up, plus the features already built into the CT and CR models, no advantage would be gained by undertaking such an effort, at this time. Thus, problems related to the need for, and the availability of, any data required to build a new model are nonexistent. However, a related problem that has not been discussed involves the manner in which $\mathrm{CO}_{2}$-induced yield effects are to be included in normative models. In Section 6.2.1 it was suggested that sufficient data appear to exist in order to build a yield-climate response model directly into the framework of existing econometric models. However, it is not possible to include these effects directly into a linear programing model without introducing substantial nonlinearities in the constraint matrix. Therefore, simulated yield changes will have to be dealt with by changing the yield coefficients within the model, consistent with the values that are arrived at by other methods. Nevertheless, to do even this will require a method for systematically disaggregating what presumably will be a set of yield estimates for fairly large regions down to the producing region level contained within the basic CARD model.

Statistical Accuracy and Model Stability. Normative models, by definition, provide estimates of potential, and not actual, production, resource use and income. Therefore, the issue of statistical accuracy is not directly relevant to the CT model, although it may be of some importance in evaluating the $C R$ model. Unfortunately, there are no published results showing even the relative accuracy of the CR model compared to the econometric mode 1 embedded within it on an export basis. The same basic situation holds true for the criterion of model stability. The CT model is not dynamic. While the CR model is dynamic, no indication of its stability is provided in existing documentation. 


\subsection{MODEL SUMMARY}

The selection of an agricultural sector model, or models, to conduct the analysis of the direct economic impacts associated with changes in the ambient concentration of $\mathrm{CO}_{2}$ involves two types of decisions. The first of these relates to the type of model, positive or normative, that is most nearly consistent with research objectives. Presumably, this decision will be closely tied to the ability of the type of model selected to satisfy the evaluation criteria utilized in the previous sections of this chapter. $A$ summary of there criteria and the results of applying them to both econometric and normative models, in general, are shown in Table 6.2. The second involves the selection of an actual model, or models, from within these categories. The remainder of this section will focus primarily on the first type of decision. Selecting specific models is more difficult, due largely to the inadequacy of published documentation on most of the models reviewed. In veiw of this problem, the recommendations in this chapter should, in that sense, be regarded as conditional upon the material that has been reviewed.

\section{TABLE 6.2. Sumary of Agricultural Sector Model Characteristics}

\section{Evaluation Criteria}

Ability to Model Dynamic Behavior in Agricultura 1 Markets

Ability to Model Impacts in a Stochastic Framework

Ability to Account for RiskAverse Behavior

Regiona 1 Disaggregation

Commod it. $\mathrm{D}$ is aggregation

Linkages to the Rest of the Wor ld

Data Needs and Availability

Statistical Accuracy

Model Stabflity
Model Type

\begin{tabular}{|c|c|}
\hline Econometric & Normative \\
\hline $\begin{array}{l}\text { Virtually all contain recursive- } \\
\text { dynamic features }\end{array}$ & $\begin{array}{l}\text { Only recursive programming } \\
\text { models are dynamic }\end{array}$ \\
\hline Yes, through Monte Carlo methods & Yes, through Monte Car lo methods \\
\hline $\begin{array}{l}\text { Yes, through adaptive experta- } \\
\text { tions sub-model }\end{array}$ & $\begin{array}{l}\text { Possible only in adaptive pro- } \\
\text { gramming models }\end{array}$ \\
\hline $\begin{array}{l}\text { Only one multi-regional model } \\
\text { available }\end{array}$ & $\begin{array}{l}\text { All models reviewed are multi- } \\
\text { regional }\end{array}$ \\
\hline $\begin{array}{l}\text { Food and feed grains, soybeans, } \\
\text { cotton and livestock plus some } \\
\text { other major commodities }\end{array}$ & $\begin{array}{l}\text { Range of crop coverage is usually } \\
\text { similar to econometric inodels }\end{array}$ \\
\hline $\begin{array}{l}\text { Export demands determined within } \\
\text { model; imports are exogenous }\end{array}$ & $\begin{array}{l}\text { Export demands determined within } \\
\text { model; imports are exogenous }\end{array}$ \\
\hline $\begin{array}{l}\text { Current models do not include } \\
\text { meteorological inputs; yields } \\
\text { must be changed exogenously }\end{array}$ & $\begin{array}{l}\text { Current models do not include } \\
\text { meteorological inputs; yields } \\
\text { must be changed exogenously }\end{array}$ \\
\hline Excellent short-term forecasts & Not applicable \\
\hline $\begin{array}{l}\text { Considerable model-to-model } \\
\text { variation }\end{array}$ & Not applicable \\
\hline
\end{tabular}


The two most important factors in selecting the type of model to be used to evaluate the direct economic impacts of $\mathrm{CO}_{2}$-induced can ultimately be reduced to two sets considerations, namely those related to:

1. the type of research strategy employed, and;

2. the scope of the impacts that are considered to be of importance.

\subsubsection{Research Strategies}

The type of model that is used to analyze the direct economic impacts due to $\mathrm{CO}_{2}$-induced yield changes will depend, in part, upon whether the analysis is conducted in a static or a dynamic research stragegy. In the former type of analysis, changes in crop yields are treated as if they occurred instantaneously, whereas in the latter an effort is made to simulate the effects of gradual yield changes over time. The use of a static type of approach does not imply that actual yield changes will, in fact, occur very suddenly. The rationale underlying this strategy is that by imposing this kind of an exogenous shock on the agricultural sector, one can examine how that sector would behave under existing short-run technological and institutional constraints. The results obtained from this procedure would then represent a "worst case" impact scenario. The purpose of conducting this kind of analysis is not to mislead or frighten people, but rather to place a lower bound on net benefits (or an upper bound on costs) that might be expected to occur. The dynamic strategy, on the other hand, is used to gain a better understanding of how the agricultural sector would adjust over time to gradual changes in the ambient concentration of $\mathrm{CO}_{2}$. This is a more faithful approach to reality from a conceptual standpoint, but there are important methodological difficulties associated with its use--a point that will be made clear shortly.

Both econometric and linear programing based models can be used in conjunction with the static strategy. However, the natures of the impacts that these two types of models will predict are substantially different. The parameters of an econometric model are obtained by fitting the equations in the model to recent historical data, using advanced multi-variate regression methods. As a result, these parameters reflect the relatively limited ability of the economy to adjust to sharp changes in economic conditions, given 
current technology and the inertial effect of technological and institutional rigidities on the mobility of the factors of production. Linear programming based models, on the other hand, show the optimal adjustments that will occur within the agricultural sector to exogenous shocks given perfectly rational behavior and perfect information. Thus, even if the technological parameters in the model reflect current technology and the resource constraints are consistent with reality, these models will, by definition, show the economy responding more efficiently to changes in economic conditions than will econometric models. In essence, then, an econometric model used in a static framework will predict how today's agricultural sector might actually respond to a sudden change in yields, while a linear programing based model will show the potential of today's agricultural economy to respond to these changes under perfectly competitive conditions. Differences between the projections made by these two types of models should be viewed in that light and not from the standpoint of their relative accuracy in predicting the impacts of hypothetical yield changes.

The dynamic strategy requires the use of models in which the values of some endogenous variables that are determined in one period are then used as exogenous variables (to determine the values of other endogenous variables) in the next period. A model that is built along these lines is said to be recursive. All of the econometric models reviewed for this report are recursive through the incorporation of stock adjustment and/or adaptive expectations features within them. Another class of models that contain dynamic features are recursive programming models, which employ an econometric agricultural sector model to update the price and cost parameters and the flexibility constraints within a comparison linear programming model.

While dynamic models have the ability to simulate the behavior of the agricultural sector over time, a great deal of caution must be exercised in viewing the results that are obtained from these models when the simulation period is very long. As was mentioned earlier, the parameters of econometric models tend to reflect the ability of the economy to make short-run adjustments to changes in economic conditions. Over longer periods of time, technological and institutional rigidities become less important barriers to 
adjustment; the factors of production are more responsive to own and cross price changes, and new technologies diffuse through the economy. Because the parameters of econometric models are estimated using relatively recent data, they do not reflect the ability of the economy to adjust more smoothly to exogenous shocks, given a long enough time span, and they can never capture the effects of technologies which do not exist. Therefore, the impacts that are predicted by econometric models will invariably be "frozen" in a short-run framework, consistent with the ability of today's economy to adjust to gradual changes in the future concentration of $\mathrm{CO}_{2}$ in the atmosphere. This may not be as true for recursive programming models, since they contain a normative component. Unfortunately, there is no way to see if this is indeed the case, since the data set necessary to conduct such an analysis does not yet exist.

Whichever strategy is employed, the predicted impacts for a hypothetical set of yield changes will probably be more severe then the actual impacts that would occur as a result of those same changes. Thus, accuracy is not an appropriate criteria to use in selecting which strategy to use. The advantage of the dynamic strategy is that, properly qualified, it can be used to give a better indication of the timing associated with the severity of the impacts that might occur. For example, if severe impacts do not occur until 50 years from now, then there may be very few incentives for decision-makers in either the public or the private sector to take remedial actions to address these problems in the near future. On the other hand, if these same impacts are predicted to start occurring within 10 to 20 years, then the effect of discounting will not be severe, and some actions may be appropriate. Another advantage of the dynamic strategy is that, if it is conducted periodically over time, it will provide decision-makers with information that can be used to te 11 how well the agricultural sector has been moving along the necessary adjustment path to cope with these impacts. Thus, information that is generated using the dynamic strategy can be employed in an adaptive framework to revise earlier decisions.

\subsubsection{Scope of Impacts}

Considerations associated with the scope of impacts include the level of regional-commodity detail that is possible, as well as the type of impacts 
that can be projected by the two classes of models. Because $\mathrm{CO}_{2}$-induced environmental changes will probably be distributed very unevenly on a geographic basis, there are likely to be some resulting changes to the existing geographic distribution of cropland that would not have occurred otherwise. National models, which do not include a spatial equilbrium framework, do not represent a very appropriate tool for analyzing these kinds of impacts. In general, it is a much easier task to build a large number of regions into a linear programing based model than an econometric model. This is amply attested to by the fact that the current generation CARD linear programming model contains 105 production regions, while the AGSIM econometric model contains on $1 y 13$ production regions. Greater regional disaggregation can always be defended on the ground that it is more faithful to the substantial degree of regional heterogeneity that exists with respect to climate, soil types, farm practices and farm structure. However, given the problems of forecasting the impacts of very uncertain and very distant types of environmental changes it can be argued that, despite the aggregation problem, the difference between 105 and 13 regions is of relatively little importance at this time.

A more important issue in this regard relates to the effect which the basic differences between normative and positive models will have on the magnitude of the shifts that are projected by the two methods. First of all, because linear programing based models provide normative solutions, land is freer to move among competing crops in these models than is actualiy the case. Secondly, even though land may be potentially more mobile between crops, existing economic conditions may not have tested that potential. Thus, the actual short-run responsiveness of 1 and to changes in crop prices may actually be higher than is reflected in the parameters of an econometric mode1. As a result, projections based on these estimated parameters would underestimate the true mobility of land to switch from one use to another. (However, the reverse is not true.) In general, then, one can expect that normative models will generally show substantially larger shifts in the geographic redistribution of cropland than will regionalized, econometric agricultural sector models. Presumably, these shifts would more nearly reflect the potential (although still short-run) mobility of land. 
Arother important consideration in this area involves the nature of the different types of impacts about which these two classes of models can provide information. In most cases, the output of econometric agricultural sector models is limited to periodic values for acreage planted and harvested, production, demand, various types of year end stocks and prices, by commodity, along with the values of additional variables that can be computed from this information. By contrast, the CARD based general equilibrium model can provide all of this information (including stocks, if an appropriate farm price is established), plus showing changes related to land development, transfers of 1 and between irrigated and dryland farming practices, the development of additional water supplies and inter-basin transfers of water.

This type of depth and detail is not possible within an econometric framework. This is unfortunate because many of the potential economic "problems" that have been associated with the effects of $\mathrm{CO}_{2}$ in the general literature (Kellog and Schware 1981) relate directly to these kinds of impacts. Whether these types of impacts will actually occur represents a series of testable hypotheses which can only be explored on a systematic basis through the use of normative models of the agricultural sector.

Finally, normative models are much more flexible in terms of the ease with which alternative assumptions can be built into them regarding changes in technology, production and transportation costs and resource availability. While it is always possible to change the value of exogenous variables in an econometric model during the course of simulation runs, tinkering with model parameters to reflect changes in the responsiveness of 1 and and other inputs to economic conditions is fraught with difficulties. In the case of normative models, however, these changes can be made by rescaling the technology coefficients consistent with the available information regarding the resource requirements associated with new or envisioned technologies. A great deal of caution must be exercised in doing this because it is always possible to construct a hypothetical technological fix that will make almost any potential problem within the economy disappear. By the same token, however, normative models do allow one to investigate the foreseeable technological changes (such as through yield improvements) that would have to occur in order to render 
potentially severe economic impacts relatively benign. This amounts to a kind of crystal ball gazing in which most economists usually do not indulge; however, given the unusual characteristics of the $\mathrm{CO}_{2}$ problem some investigation along these lines may be warranted.

\subsubsection{Recommendations}

Because normative and econometric models can both be used in static analysis of the direct economic impacts due to increases in the ambient concentration of $\mathrm{CO}_{2}$, while only econometric or recursive programing models can be used in a dynamic setting, two models have been selected. The choice of a normative model to conduct the static analysis is straightforward. The only linear programming based general equilibrium model of the agricultural sector is the Iowa State University CARD model, formally known as the ISU/NRE National Regional LP Model, and referred to in the body of this report as the CT (CARD Tatonnement) mode1. This model is the product of over 20 years of on-going development, and has been extensively used in making long-range projections for USDA, the Water Resources Council and EPA. The only serious drawback to its use is the cost of running the model. Initial runs vary in cost in an area from $\$ 1200$ to $\$ 1600$. Subsequent runs become less expensive, although cost varies depending upon the number of changes made between runs.

The econometric model selected is the AGSIM regional model. While the structure of AGSIM is in some ways less sophisticated than the structure of some of the other models examined, it was the only econometric model surveyed that is built explicitly around a spatial equilibrium framework. Other attributes that make it attractive are its apparent dynamic stability, the unusual fidelity to economic theory that is contained within the model and the systematic way in which a priori information is incorporated into the estimation of model equations, the relative ease with which the yield equations in it can be re-estimated to incorporate the effects of meteorological variables and, finally, the fact that technological changes can be incorporated explicitly into the model by changing parameters that will not demonstratably affect the stability of the model, nor result in perverse economic behavior. It should be pointed out, however, that despite these features which make it attractive in the current framework, AGSIM remains 
essentially a short-term forecasting model in its design. An alternative econometric model, NIRAP, used by USDA to make some of its long-term projections is available. Unfortunately, there is very little substantive documentation on NIRAP. The documentation that does exist is in the form of an initial draft, (a) which is sufficiently obscure about the structure of this model in certain areas as to raise serious questions about its overall quality.

Table 6.3 compares the important structural features of the AGSIM and CT models and indicates possible changes that could be made to AGSIM in order to improve the quality of the information that this model can provide about the possible impacts of $\mathrm{CO}_{2}$-induced environmental changes on the agricultural sector. Recommended changes to the AGSIM model include modifications in the following areas:

- Stochastic Features. AGSIM is a deterministic model. It can be made stochastic by incorporating within its a framework a simulator that would first draw a series of random deviates from the distributions of the yield equation error terms and then simulate the impact of the drawn values on the endogenous variables within the model. An example to follow in the construction of this simulator is contained within the model POLYSIM (Ray and Richardson 1978).

- Incorporation of Producer Risk-Preference. AGSIM currently contains no features which permit an analysis of the impact of the risk-averse behavior of producers on agricultural production. This feature can be built into the model by re-estimating the relevant acreage equations using the approaches recommended by Behrman (1968) or Just (1974, 1975).

- Incorporation of Meteorological Variables. The yield equations in AGSIM do not take into account the impact of variations in weather upon crop production. This feature of the model can be modified by re-estimating the regional yield equations based on the structure shown in Equation (6.23). Such a change would amount to embedding a set of statistical crop yield models within the existing model structure. The advantages of doing this, as opposed to using a separate set of crop yield models are

(a) Quance, L. 1980. The National Inter-Regional Agricultural Projections (NIRAP) System: An Executive Briefing. Trade Policy Systems, Trade Policy Branch, International Economics Division, Economics, Statistics, and Cooperatives Service, U.S. Department of Agriculture, Washington, D.C. 
TABLE 6.3. Summary of Structural Features and Recommended Changes to AGSIM and CARD Tatonnenment Models

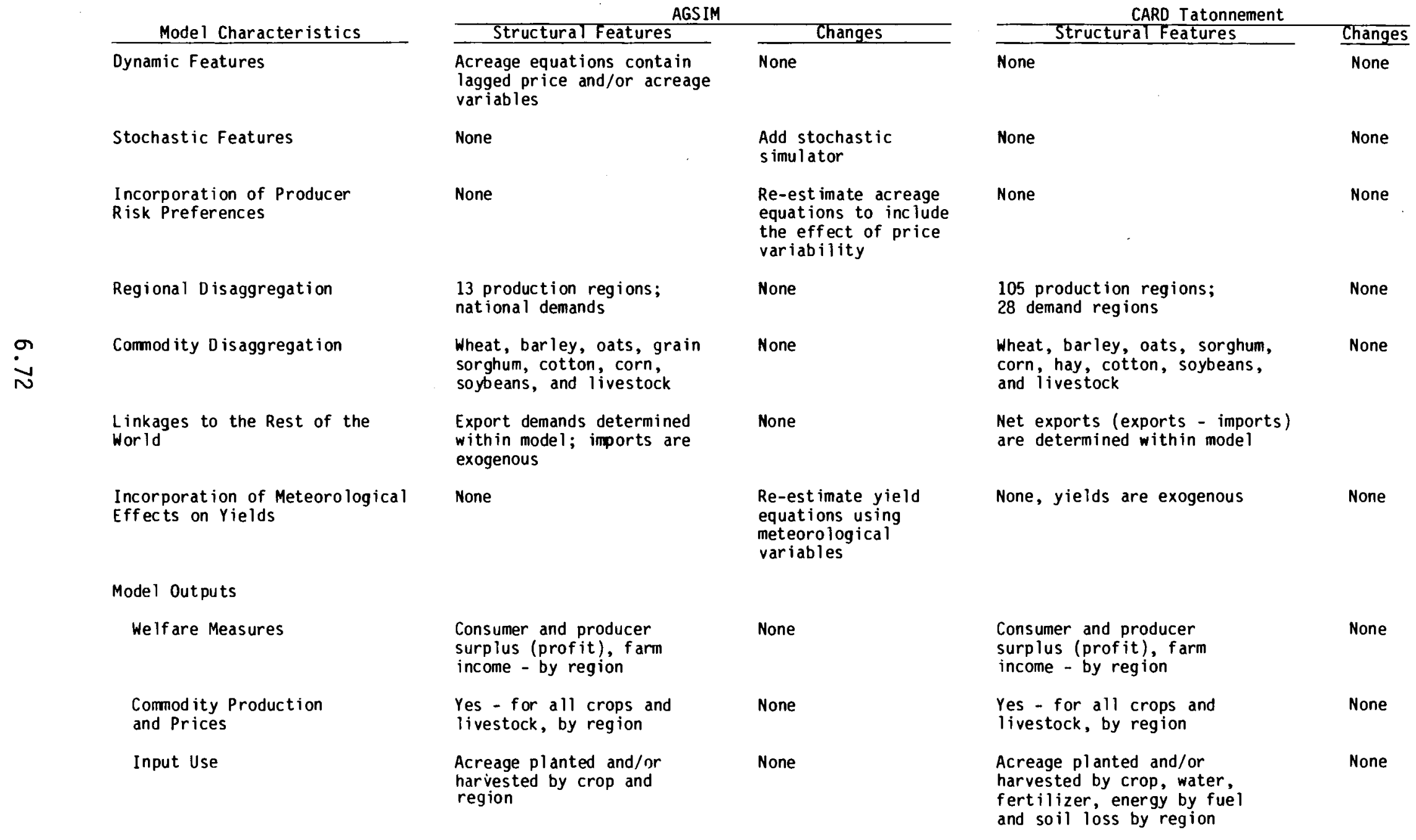


that (a) the effect of weather on crop yields is not a priori assumed to be independent of economic forces and (b) stochastic simulations can be performed using specific information about the variability in meteorological phenomena, apart from other sources of randomness.

No changes are contemplated for the CT model. While it would, in theory, be possible to make the CT model dynamic and to include the impact of producer risk preferences in the objective function of the model, it is not clear that the relatively large research and operational costs associated with these improvements can be justified in terms of the increased quality of the model's performance. More importantly, adding these features would effectively distort the usual, normative connotations attached to the optional solutions obtained from mathematical programming models by introducing the effect of institutional, technological and perceptual lags on the behavior of economic agents within the agricultural sector. In view of these considerations, it is recommended that the $C T$ model be used, without modification, to examine how today's agricultural sector would adjust, strictly from a normative standpoint, to a one-time exogenous shock caused by $\mathrm{CO}_{2}$-induced changes in crop yields. The information provided by such an exercise would be extremely useful in concert with the forecast provided by AGSIM within an instantaneous adjustment framework in bounding the so-called worse-case scenario.

Neither of the models selected above are well suited to analysis of the economic impacts that might be expected to occur within the framework of international trade. At this time, the best available model to analyze these impacts is probably the world grains, 0 ilseed and Livestock (GOL) Model currently maintained by USDA. Another possible alternative, which is in the process of being built is the NIRAA-IIASA-MSU or (NIIM) Model, which is a U.S. agricultural sector model being assembled to fit into the world agricultural model system currently being developed at the Institute for Applied Systems Analysis in Austria. NIIM and the IIASA system are intended for mid- to long-term world projections and policy analysis. The IIASA system consists of a world trade, spatial equilibrium algorithm connected to a set of simplified regional models including Europe, Japan, the rest of Asia, the socialist world, and other major exporters and importers of agricultural commodities. Completion of the entire system is expected to take another one to three years. 


\section{REFERENCES}

Adams, B. M. 1976. Economic Effect on Agricultural Production of Alternative Energy Prices: Texas High Plains. Technical Report No. 73, Texas Water Resources Institute, Texas A\&M University, College Station, Texas.

Anstrom, K. 1970. Introduction to Stochastic Control Theory. Academic Press, New York, New York.

Baumes, H. S. and W. H. Meyers. 1980. The Crops Model: Structural Equations, Definitions, and Selected Impact Multipliers. NED Staff Report, Economics, Statistics and Cooperatives Service, U.S. Department of Agriculture, Washington, D.C.

Behrman, J. R. 1968. Supply Response in Underdeveloped Agriculture. North Holland, Amsterdam, The Netherlands.

Ben-David, S. and W. Schulze. 1975. "Economic Impact of Climatic Change on World Agriculture: Benefit-Cost Analys is for Cotton and Corn." In Economic and Social Measures of Biologic and Climate Change, CIAP Monograph 6, pp. 5.3-5.43. U.S. Department of Transportation, DOT-TST-75-56, National Technical Information Service, Springfield, Virginia.

Binswanger et al. 1978. Induced Innovation, Technology, Institutions, and Development. The John Hopk ins University Press, Baltimore, MaryTand.

Cagan, P. 1956. "The Monetary Dynamics of Hyper-Inflations." In Studies in the Quantity Theory of Money, ed. M. Freedmand, pp. 128-176. University of Chicago Press, Chicago, Illino is.

Cappi, C. et al. 1978. "A Model of Agricultural Production and Trade in Central America." In Economic Integration in Central America, eds. W. Cline and $E$. Delgado, The Brookings Institution, Washington, D.C.

Chow, G. C. 1967. "Multiplier, Accelerator, and Liquidity Preference in the Determination of National Income in the United States." Review of Economics and Statistics $49(2): 241-261$.

Collins, G. S. 1980. "An Econometric Simulation Model for Evaluating Economic Impacts of Technological Change on Major U.S. Field Crops." Ph.D. Thes is, Texas A\&M University, College Station, Texas.

Daniel, R. and D. Steadman. 1981. The Chase Econometrics U.S. Agricultural Model, a Technical Discussion. Chase Econometrics, Bala Cynwyd, Pennsylvania.

Day, R. H. 1963. "On Aggregating Linear Programing Models of Production." Journal of Farm Economics 45(4):797-813. 
Dean, G. W. and H. 0. Carter. 1961. Guides to Profitable Cropping Systems for Yolo County Farms. Giannini Formation Research Report No. 242, University of California, Berkeley, California.

Doll, J.P. 1967. "An Analytical Technique for Estimating Weather Indices from Meteorological Measurements." Journal of Farm Economics 49(1):79-88.

Dvoskin, D. et al. 1978. Energy Use in U.S. Agriculture: An Evaluation of National and Regional Impacts from Alternative Energy Policies. CARD Report No. 78, Center for Agricultural and Rural Development, Iowa State University, Ames, Iowa.

Duloy, J. H. and R. D. Norton. 1973a. "The CHAC Demand Structure." In Programming Studies for Mexican Agricultural Policy, eds., R. D. Norton and L. M. Solis, pp. 118-142. International Bank for Reconstruction and Development, Development Research Center, Washington, D.C.

Duloy, J. H. and R. D. Norton. 1973b. "CHAC: A Programing Model of Mexican Agriculture." In Multilevel Planning: Case Studies in Mexico, eds. L. Groeux and A. Manne, pp. 291-337. North Holland, Amsterdam, The Netherlands.

Duloy, J. H. and R. D. Norton. 1975. "Prices and Incomes in Linear Programming Models." American Journal of Agricultural Economics 57(4):591-600.

English, B. C. et al. 1981a. A Documentation of the Rural Conservation Act's Assessment Model of Regional Agricultural Production, Land and Water Use, and Soil Loss. 2 Vols., Forthcoming CARD Report, Center for Agricultural and Rural Development, Iowa State University, Ames, Iowa.

English, B. C. et al. 1981b. "Tatonnement Modeling: A Variation to Linear Programing." Paper presented at the Annual Meetings of the American Agricultural Economics Association, July 26-29, Clemson University, Clemson, South Carolina.

Golberger, A. S. 1959. Impact Multipliers and the Dynamic Properties of the Klein-Goldberger Model. North Holland, Amsterdam, The Netherlands.

Hammersley, J. M. and D. C. Handscomb. 1964. Monte Carlo Methods. Methuen, London, Engl and.

Hayami, Y. and V. W. Ruttan. 1971. Agricultural Development: An International Perspective. Johns Hopkins University Press, Baltimore, Maryl and.

Heady, E. 0. and W. Candler. 1964. Linear Programing Methods. Iowa State University, Ames, Iowa.

Just, R. E. 1974. "An Investigation of the Importance of Risk in Farmer's Decisions." American Journal of Agricultural Economics 56(1):14-25. 
Just, R. E. 1975. "Risk Response Models and The ir Use in Agricultural Policy Evaluation." American Journal of Agricultural Economics 57(5):836-843.

Kellog, W. W. and Robert Schware. 1981. Climate Change and Society, Consequences of Increasing Atmospheric Carbon Dioxide. Westview Press, Boulder, Colorado.

Langley, J. A. et al. 1981. A Recursive Adaptive Hybrid Model for the Analys is of the National and Interregional Impacts of Three Alternative Agricultural Situations. CARD Report No. 100. Center for Agricultural and Rural Development, Iowa State University, Ames, Iowa.

Mayo, D. and J. McMillan. 1975. "Economic Estimates of the Climate Induced Changes in World Wheat Production." In Economic and Social Measures of Biologic and Climate Change. CIAP Monograph No. 6, pp. 5.102-5.145. U.S. Department of Transportation, DOT-TST-75-56, National Technical Information Service, Springfield, Virginia.

McCar 1, B. A. and T. H. Spreen. 1980. "Price Endogenous Mathematical Programming as a Tool for Sector Analysis." American Journal of Agricultural Economics 62(1):87-102.

Meister, A. D. and K. J. Nicol. 1975. A Documentation of the National Water Assessment Model of Regional Agricultural Production, Land and Water Use, and Environmental Interaction. Center for Agricultural and Rural Development, Iowa State University, Ames, I owa.

Meister, A. D. et al. 1978. Quadratic Programing Models Applied to Agriculture. Iowa State University, Ames, Iowa.

Nerlove, M. 1958. Distributed Lags and Demand Analysis for Agricultural and Other Commodities. Agricultural Handbook No. 141, Agricultural Marketing Service, U.S. Department of Agriculture, Government Printing Office, Washington, D.C.

Nicol, K. J. and E. 0. Heady. 1976. A Model for Regional Agricultural

Analys is of Land and Water Use, Agricultural Structure, and the Environment: A Documentation. Center for Agricultural and Rura 1 Development, Iowa State University, Ames, Iowa.

Patton, W. P. 1980. "The Impact of the 1977 Food and Agriculture Act on Cotton Production in the United States: A Simulation of Policy Alternatives." Ph.D. Thesis, Texas A\&M University, College Station, Texas.

Pindyck, R. S. and D. Rubinfeld. 1976. Econometric Models and Economic Forecasts. McGraw-Hill Book Company, New York, New York. 
Ray, D. E. 1971. "An Econometric Simulation Model of the United States." 2 Vols. Ph.D. Thesis, Iowa State University, Ames, Iowa.

Ray, D. E. and J. W. Richardson. 1978. Detailed Description of POLYSIM. Technical Bulletin T-151, Agricultural Experiment Station, Oklahoma State University, Stiliwater, Oklahoma.

Reynolds, T. M. and E. 0. Heady. 1975. Alternative Futures for American Agricultural Structure, Policies, Income, Employment, and Exports: A Recursive Simulation. CARD Report No. 56. Center for Agricultural and Rura1 Development, Ames, Iowa.

Schmitz, et al. 1975. "Climatic Changes and Agriculture: A Case Study on Wheat." In Economic and Social Measures of Biologic and Climate Change. CIAP Monograph No. 6, pp. 5.44-5.76. U.S. Department of Transportation, DOT-TST-75-56, National Technical Information Service, Springfield, Virginia.

Shumway, C. R. et al. 1970. Regional Resource Use for Agricultural Production in California, 1961-65. Gianinni Foundation Mongraph No. 25, University of California, Berkeley, California.

Simmons, R. L. and C. Pomareda. 1975. "Equilibrium Quantity and Timing of Mexican Vegetable Exports." American Journal of Agricultural Economics $57(3): 472-479$.

Stailings, J. C. 1961. "A Measure of the Influence of Weather on Crop Production." Journal of Farm Economics 43(4)1153-1160.

Takayama, T. and G. G. Judge. 1971. Spatial and Temporal Price and Allocation Models. North Holland, Amsterdam, The Netherlands.

Taylor, C. R. et al. 1976. A Description of Two National Spatial-Equilibrium Mode is of Crop Production. Agricultural Economics Research Report AERR-1. Department of Agricultural Economics, University of Illinois, Champaign-Urbana, I1lino is.

USDA. 1978. Alternative Futures for World Food in 1985. 3 Vols. Foreign Agricultural Economics Report No. 151, U.S. Department of Agriculture, U.S. Government Printing Office, Washington, D.C.

USDA. 1981. Agricultural and Other Economic Models of the Economics and Statistics Service. Economics and Statics Service, U.S. Department of Agriculture, Washington, D.C.

Wade, J. C. and E. 0. Heady. 1976. A National Model of Sediment and Water Quality: Various Impacts on American Agriculture. CARD Report N0. 67. Center for Agricultural and Rural Development, Iowa State University, Ames, Iowa. 
Watt, D. L. 1981. "Implications for U.S. Agriculture of Expanded Sunflower Production: A Simulation to the Year 2000." Paper presented at the Annual Meeting of the Western Agricultural Economics Association, August 23-27, Lincoln, Nebraska.

Webb, S. B. 1981. "The Impact of Increased Alcohol Production on the Agricultural Sector: A Simulation Study." American Journal of Agricultural Economics 63(3):532-537.

Weiss, M. D. 1979. An Automated Weather/Yield System: Development, Structure and Function. Commodity Economics Division, Economics Statistics, and Cooperatives Service, U.S. Department of Agriculture, Washington, D.C. 


\subsection{ANALYSIS OF METHODS AND MODELS FOR ASSESSING THE INDIRECT IMPACTS OF $\mathrm{CO}_{2}$-INDUCED ENV IRONMENTAL CHANGES ON OTHER SECTORS OF THE U.S. ECONOMY}

As pointed out in Section 3.4 of this report, the impacts of $\mathrm{CO}_{2}$-induced environmental changes within the agricultural sector can be expected to spill over into other sectors of the economy. Moreover, because of the complex linkages between industries that are tied either directly or indirectly to the agricultural sector, it is not possible to determine on a priori basis which industries will be most affected. As a result, it will be necessary to use existing models or develop new ones which track the primary impacts of $\mathrm{CO}_{2}$-induced environmental changes within the agricultural sector through the rest of the economy along two different paths. The first of these involves following the effects of $\mathrm{CO}_{2}$ buildup back through the input and factor markets which supply the agricultural sector, and from these markets forward through their sales to other industries and ultimately to industries that sell to final demand. The second path is somewhat more direct: it runs from those industries which convert agricultural products for other intermediate uses, and then to final demands.

While the exact nature of these flows may be difficult to grasp from a conceptual standpoint, there are many different kinds of models that can be used to determine the effect which economic changes in one sector of the economy will have upon the price and/or output levels which prevail in other sectors of the economy. The first section of this chapter presents an analys is of the alternative types of models that can be used to do this.

There are indications that changes in the ambient concentration of $\mathrm{CO}_{2}$ may have a significant impact on the current geographic distribution of water resource supplies, as well as upon the interindustry allocation of existing water and land resources. Models that can be used to analyze these kinds of impacts in a competitive market framework will be examined in Section 7.2. Because $\mathrm{CO}_{2}$ buildup has the potential to directly and indirectly influence the prices which consumers will pay in the future for a great many goods and 
services, Section 7.3 will examine different ways of measuring the impact of these price changes on the welfare of consumers. Finally, Section 7.4 will summarize the strengths and weaknesses of the models reviewed in previous sections and make specific recommendations regarding the use of selected models in future research.

Before continuing, one very important point must be made about the theoretical implications associated with linking economy-wide models to agricultural sector models in order to conduct the indirect impact analysis phase of the research. In a perfectly competitive general equilibrium framework, exogenous changes from outside the economic system which in any way affect one market will simultaneously affect economic behavior in other markets within the economy that are in any way connected, either directly or indirectly, to the original market. This is synonymous with the economic aphorism that ultimately everything depends on everything else. If that is true, then there will be very few economic agents whose behavior will not in some way be touched by changes in the ambient concentration of $\mathrm{CO}_{2}$.

This suggests that, in order to be fully faithful to the concept of general equilibrium, all of the linkages and feedback loops within the economy which determine the behavior of individual producers ought to be closed. However, the practice of using one model to determine the direct economic impacts associated with $\mathrm{CO}_{2}$ buildup and another model to calculate the indirect impacts of these changes on the rest of the economy directly violates the assumption of closure which is so important in a general equilibrium framework. In practice the only way this problem can be solved is to develop a single model of the economy in which a very detailed agricultural sector is fully contained.

This latter approach may be possible. However, implementing such an approach would involve further research to investigate the feasibility of doing this, and, once that research was completed, it is more likely than not that the findings would require the development of an entirely new model of the economy with extensive detail given over to the supply side of the 
agricultural economy and its connections with input and factor markets. But that is beside the point for the moment, because at this time it is not clear, even if this marriage could be accomplished, that it would be worthwhile--for practical, as opposed to theoretical, reasons.

Our decision to approach the entire problem of impact analysis from a partial equilibrium perspective was made on the basis that the advantages which could be gained by the use of a disaggregated, multi-regional agricultural sector model outweighed the disadvantages of approaching the problem from a partial equilibrium framework. The net advantage of the partial equilibrium approach rests on three considerations. These are:

1. It is well within the capability of existing agricultural sector models to simulate the effects of environmental changes on the yields of individual crops within many different regions, whereas this is unthinkable in existing economy-wide general equilibrium mode ls.

2. The use of multi-regional agricultural sector models allows for the analysis of geographic shifts in the distribution of agricultural production which is a likely possibility in the event of very dramatic $\mathrm{CO}_{2}$-induced changes in the natural and physical environments in which agricultural production takes place. This is not possible within the framework of existing national models, with the single exception of multi-regional econometric and normative models. However, these models really do not constitute general equilibrium models in the pure sense, because they include only a single sector.

3. The costs of building sufficient detail into a national general equilibrium model consistent with the current capabilities of agricultural sector models in (1) and (2) above would be high. Furthermore, if such an investment is justified, support for building such a model will likely come from projections developed by less sophisticated methods. 
In that general framework, the following section is devoted to exploring the use of existing macro-models models in conjunction with agricultural sector models to analyze the indirect impacts of $\mathrm{CO}_{2}$-induced environmental changes in the agricultural sector.

\subsection{ANALYSIS OF ALTERNATIVE METHODS FOR MODEL ING THE NATIONAL ECONOMY}

There are esssentially four different approaches that can be taken in building a model of the U.S. economy that could prove helpful in evaluating the indirect impacts of $\mathrm{CO}_{2}$-induced environmental changes in the agricultural sector. They include:

- input-output models

- input-output based econometric models

- normative models

- multi-regional econometric models

\subsubsection{Input-Output Mode 1s}

The most straightforward of all of the different kinds of general equilibrium, multi-sector models are input-output models. The classic input-output model, based on the work of Leontief (1951), seeks to answer a very specific kind of question, namely: what level of output should each of the industries (or sectors) in an economy produce in order to just satisfy the total demand for each industry's output?

Since this is a fairly restrictive question, it may be assumed that models used to solve this particular problem are constructed on the basis of an equally restrictive set of assumptions. And, indeed, this is so. As a rule, the following assumptions are traditionally incorporated within the framework of a static input-output model:

1. Each industry either produces a single homogeneous good or, if it produces more than one good, these goods must be produced in fixed proportions.

2. Each industry uses a fixed input ratio for the production of its output. 
3. Production in each industry is subject to constant returns to scale, such that a $1 \%$ increase in all inputs will result in a $1 \%$ increase in output.

4. The marginal cost of producing each additional unit of output is constant in each industry (perfectly elastic supplies).

5. Final demands for goods and services are exogenous to the system (perfectly inelastic demands).

Consistent with this set of assumptions, the structure of input-output economy can be described in terms of a transactions table such as the one shown in Table 7.1 for an economy that contains $n$ sectors.

TABLE 7.1. Transactions Table

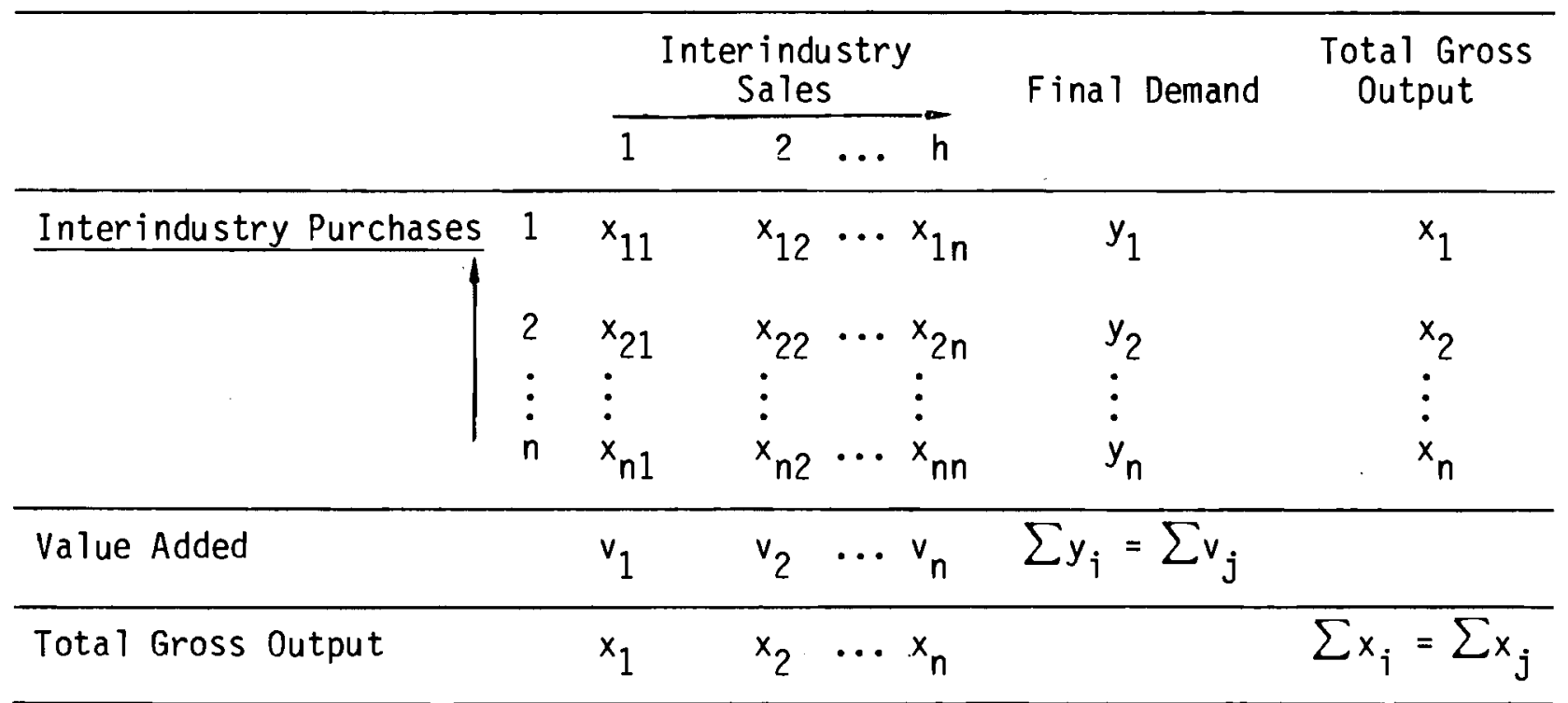

Input-output analysis divides the economy into two major components, namely: suppliers (or sellers) and purchasers (or users). Suppliers include:

1. Intermediate industries which purchase inputs and process them into outputs which are, in turn, sold to other intermediate industries or to final users. The sales of this group appear as interindustry sales in Table 7.1 . 
2. Primary suppliers composed of hou seholds and other types of factor owners whose output is not directly dependent on purchased inputs. The sales of this group are represented by the value added row in Table 7.1

Purchasers include:

1. Intermediate industries which buy the outputs of supplying industries for use as inputs for further processing. The purchases of this group appear as interindustry purchases in Table 7.1.

2. Final purchasers who buy the goods and services produced by intermediate industries for final consumption. This group is represented by the final demand column in Table 7.1.

The transactions table is transformed into a behavioral model by assuming that the purchases of intermediate products from industry (i) are proportional to the level of gross output in $(j)$. Consequently,

$$
x_{i j}=a_{i j} x_{j}
$$

which implies a fixed proportions production function. Accepting this assumption of fixed proportions production function allows one to proceed to the construction of the table of technical coefficients. This table is obtained by dividing each interindustry sale of the transactions table by the gross output level of the purchasing industry. Therefore,

$$
a_{i j}=x_{i j} / x_{j}
$$

defines the input coefficients. This is denoted as the A matrix. Each of the coefficients $\left(a_{i j}\right)$ in the $A$ matrix, below represents the partial cost of the inputs supplied by industry $i$ to industry $j$ per dollars worth of $j$ 's gross output. 
Matrix of Technological Coefficients

\begin{tabular}{c|cccc} 
& 1 & \multicolumn{1}{c}{2} & $\ldots$ & $n$ \\
\hline 1 & $a_{11}$ & $a_{12}$ & $\ldots$ & $a_{1 n}$ \\
2 & $a_{21}$ & $a_{22}$ & $\ldots$ & $a_{2 n}$ \\
$\vdots$ & $\vdots$ & $\vdots$ & & $\vdots$ \\
$n$ & $a_{n 1}$ & $a_{n 2}$ & $\cdots$ & $a_{n n}$
\end{tabular}

Consistent with these assumptions, the total output of the ith industry in an economy composed of a sectors can be expressed as

$$
x_{i}=\sum_{j=1}^{n} a_{i j} x_{j}+y_{i}
$$

where $x_{i}$ is the gross output of the ith industry, and $y_{i}$ is final demand ior the output of the ith industry.

Equation (7.1) can be rewritten as

$$
\left(1-a_{i j}\right)-\sum_{j=2}^{n} a_{i j} x_{j}=y_{i}
$$

Thus, the interindustry flow of goods and services within the economy can be expressed by the following system of $n$ linear equations

$$
\begin{aligned}
& \left(1-a_{11}\right) x_{1}-a_{12} x_{2}-\ldots-\quad a_{1 n} x_{n}=y_{1} \\
& -a_{21} x_{1}+\left(1-a_{22}\right) x_{2}-\ldots-\quad a_{2 n} x_{n}=y_{2} \\
& -a_{n 1} x_{1}-a_{n 2} x_{2}-\ldots+\left(1-a_{n n}\right) x_{n}=y_{3}
\end{aligned}
$$

The system of equations shown in Equation 7.3 can be expressed much more simply in matrix notation as 


$$
(I-A) X=Y
$$

where $I$ is a diagonal $n \times n$ identity matrix, $A$ is the $n \times n$ matrix of technical coefficients, $X$ is a $n \times 1$ vector of industry gross output and $Y$ is a $n \times 1$ vector of final demands. If $(I-A)$ is nonsingular, then the inverse $(I-A)^{-1}$ can be found, and Equation (7.4) will have the unique solution

$$
X=(I-A)^{-1} Y
$$

Setting $(I-A)^{-1}=C$, Equation (7.5) can written as a system of linear equations

$$
x_{i}=\sum_{j=1}^{n} c_{i j} y_{j}
$$

where each of the $c_{i j}$ coefficients (from the matrix $c$ ) represents the change in the gross output of industry $i$ due to a one dollar change in the demand of industry $j$, or

$$
c_{i j}=\frac{\Delta x_{i}}{\Delta y_{j}} \quad \text { (Industry to Industry Impact) }
$$

The sum of the coefficients in any column $j$ indicates the total change in output for all industries that results from a one dollar change in the final demand for $j$, or

$$
c_{j}=\sum_{i=1}^{n} c_{i j}=\frac{\sum_{i=1}^{n} \Delta x_{i}}{\Delta y_{j}} \quad \text { (Economy Impact) }
$$

Thus, 


$$
\Delta x=\sum_{j=1}^{n} c_{j} \Delta y_{j}
$$

Finally, the sum of the coefficients across any row $i$, weighted by the final demarid distribution vector, indicates the total change in the output of industry $i$ due to a one dollar change in the final demand for all industries, or

$$
c_{i}=\sum_{j=1}^{n} c_{i j}-\frac{y_{j}}{\sum_{j=1}^{n} y_{j}}=\frac{\Delta x_{i}}{\sum_{j=1}^{n} \Delta y_{j}} \quad \text { (Industry Impact) }
$$

Thus,

$$
\Delta x_{i}=c_{i} \sum_{j=1}^{n} y_{j}
$$

The relationships shown in Equation (7.7b) and (7.8b) are frequently utilized in impact analysis to measure changes in gross output attributable to given incremental changes in final demand. In the current research context, it would be possible, for example, to use existing input-output tables for the J.S. economy (Bureau of Economic Analysis (BEA) 1979) to determine the change in total gross output due to simulated changes in the final demand for those agricultural products included in the forecast provided by an agricultural sector model. The problem with doing this is that sales of raw agricultural commodities to final demand represents a small fraction of all sales, the largest share going to other intermediate industries. Since agricultural sector models do not project final demands for processed goods, some unquestionably arbitrary method would have to be devised for simulating these demands, as well. Otherwise, the full impact of $\mathrm{CO}_{2}$ on other sectors of the economy would be lost. 
A second possible way to approach the analysis of indirect impacts using conventional input-output models is to assume that the effects of $\mathrm{CO}_{2}$ on the agricultural sector can be characterized in the form of technological change. That is to say, increases in the ambient concentration of $\mathrm{CO}_{2}$ would have the effect of altering both the level and mix of inputs in certain agricultural industries. Consider, for example, the effect of $\mathrm{CO}_{2}$ on the input mix of a highly aggregated agricultural sector; call it sector $j$. The current production technology of that sector is characterized by the technical coefficients, $a_{i j}$, in the $j$ th column of the transactions matrix, or the vector $a_{j}$. Each element in this vector expresses the value of the impacts required from the ith intermediate industry in order to produce a dollar's worth of gross output. $\mathrm{A} \mathrm{CO}_{2}$-induced change in the technology described by this vector can be expressed as $\Delta a_{j}$. The new technical coefficients matrix consistent with the inclusion of these effects is expressed as

$$
A+\Delta a_{j} u_{j}^{\prime}
$$

where $u_{j}$ is an orthonormal vector with its $j$ th element equal to unity.

The impact of $\mathrm{CO}_{2}$ on the production technology of the jth sector could occur in one of three ways:

1. if the effect of $\mathrm{CO}_{2}$ buildup up is to reduce the productivity of some inputs, then some elements of $\Delta a_{j}$ will be positive, as larger quantities of these inputs will be required to produce one unit of j's output;

2. if the effect of $\mathrm{CO}_{2}$ buildup is to increase the productivity of some inputs, then some elements of $\Delta a_{j}$ will be negative, since a smaller amount of these inputs will be required to produce one unit of $j$ 's output; and

3. if the effect of $\mathrm{CO}_{2}$ buildup is to reduce the productivity of some inputs and increase the productivity of others, then there will be both positive and negative elements in $a_{j}$. 
In each of these cases, $\mathrm{CO}_{2}$ buildup will have a different impact on gross output, holding final demands constant. Illustrating these results requires some fairly complex manipulations in matrix algebra. In all of the three cases, the impact on the gross output of sector $j$ is

$$
\begin{aligned}
x_{j} & =\left[(I-A)-\Delta a_{j} u_{j}^{\prime}\right]-1 y=\left[(I-A)^{-1}+\frac{(I-A)^{-1} \Delta a_{j} u_{j}^{\prime}(I-A)^{-1}}{1-u_{j}^{\prime}(I-A)^{-1} \Delta a_{j}}\right] y \\
& =x+\frac{(I-A)^{-1} \Delta a_{j} x_{j}}{1-u_{j}^{\prime}(I-A)^{-1} \Delta a_{j}}
\end{aligned}
$$

which directly implies

$$
\Delta x=\frac{(I-A)^{-1} \Delta a_{j} x_{j}}{1-u_{j}^{\prime}(I-A)^{-1} \Delta a_{j}}
$$

Thus, the direction of the change in gross output, $\Delta x$, will depend on the characteristics of the vector $\Delta a_{j}$. In case 1 above, $\Delta a_{j}$ is by definition positive semi-definite. In case $2, \Delta a_{j}$ is again by definition negative semi-definite, while in case $3, \Delta a_{j}$ could be either positive or negative semi-definite.

Given this information, the following nontechnical conclusions can be drawn from Equations (7.10) and (7.11):

Case 1. $\mathrm{CO}_{2}$ buildup has the effect of increasing the input/output ratios in the agricultural sector; therefore, a larger quantity of inputs will be required to meet the same level of final demand. Thus, gross output in the entire economy will increase (holding final demands constant).

Case 2. $\mathrm{CO}_{2}$ buildup has the effect of decreasing the input/output ratios in the agricultural sector; as a result, a smaller quantity of inputs 
will be required to meet the same level of final demand. Thus, gross output in the entire economy will decrease (holding final demands constant).

Case 3. The impact of $\mathrm{CO}_{2}$ buildup on the direction of change in gross output is indeterminant. Intuitively, however, whether gross output goes up or down will depend on three factors: (a) the relative intensities associated with the use of different inputs by the agricultural sector prior to the change in atmospheric $\mathrm{CO}_{2}$, (b) the relative magnitudes of the changes in the technological coefficients due to $\mathrm{CO}_{2}$ buildup, and $(c)$ the distribution of final demands across all industries.

There are essentially two major problems with this approach. The first is a practical question, namely: from what source will the necessary information come that will permit adjustment to the technological coefficients within the agricultural sector? Agricultural sector models are not constructed to provide this type of information. If this information comes from other sources, for example from the judgment of experts (the most likely source), what assurance is there that the changes that are made to the technical coefficients will be consistent with the direct impacts that are projected? Short of determining the nature of $\mathrm{CO}_{2}$-induced changes to the magnitude of these coefficients after the fact, any attempt to explicitiy characterize the impact of $\mathrm{CO}_{2}$ buildup on the agricultural sector as a form of technological change is bound to be an arbitrary exercise.

The second problem is far more genera 1 and applies broadly to the use of input-output models for analyzing the indirect economic impacts due to future increases in the ambient concentraton of $\mathrm{CO}_{2}$. In Sections 3.3 and 3.4 , it was shown that $\mathrm{CO}_{2}$-induced changes in the environment will directly affect the equilibrium prices of agricultural commodities, as well as indirectly affecting the prices of other goods and services in the economy. $\mathrm{CO}_{2}$-induced changes in the demand for variable inputs and fixed factors will have an impact on the marginal costs of supplying these goods and services to a great many sectors of the economy due to the backward linkages from agriculture to other industries and factor owners. At the same time, $\mathrm{CO}_{2}$-induced changes in the price of agricultural commodities will affect the 
prices which consumers and other producers must pay for these commodities through forward linkages. Ultimately, then the potential exists for the effects of $\mathrm{CO}_{2}$-induced environmental changes to spill over into all of the sectors of the economy, influencing the supply and demand for all of the final goods and services that are produced. In fact, not all final demands will be affected and the magnitude of the impacts on the equilibrium prices and quantities associated with the consumption of final goods and services will vary widely. In an economy whose structure is characterized by an input-output model, prices play no role in determining the rates at which firms will substitute variable inputs and fixed factors in the production of goods and services, nor the rates at which households will substitute final goods in consumption. The former type of substitutions depend only upon the technological coefficients within the model, which are constant, while final demands are treated as given. Although the assumptions that lie behind these features of input-output models may be acceptable for looking at some $k$ inds of impacts, they are inappropriate for the analysis of the indirect economic impacts due to increases in the ambient concentration of $\mathrm{CO}_{2}$.

\subsubsection{Input-Output Based Econometric Models}

Until fairly recently, there were essentially only two alternatives for modeling the behavior of the national economy, namely: input-output models and macroeconometric models. The former type of model was traditionally used in impact analysis, while large scale macroeconomic models were used largely to measure the performance of the economy in response to changes in selected fiscal and monetary policy instruments. However, within the last decade or so, efforts have been undertaken to merge these two types of models into a framework that explicitly incorporates the interaction between output and factor markets while maintaining a high level of detail with regard to interindustry transactions within the economy. These models can be thought of in very simple terms as an input-output model to which the following additions are made:

- The traditional vector of exogenous final demands is replaced by a system of econometrically estimated final demand equations in which 
the final demand for each industry's output is a function of its own price and the prices of other final demand goods.

- The fixed coefficient demand functions for primary inputs in each column of the input-output table are replaced by nonlinear factor demand equations which characterize the demand for each primary input by the jth (column) industry as a function of industry output and the prices of these primary inputs.

- The investment demand for capital is treated in a stock adjustment framework which makes the input-output model dynamically recursive due to the lag structure inherent in this fomulation.

- Provisions are incorporated to estimate the equilibrium prices for some primary inputs, ordinarily for labor and sometimes for capital, as we 11 .

Two proprietary versions of this type of model were reviewed: the Battelle Memorial Institute (BMI) Forecasting and Simulation Model (FORSYS) and a model constructed by Wharton Econometric Forecasting AsSociates (WEFA). The remainder of this section will describe the major features of the FORSYS model. The selection of FORSYS rather than the WEFA model was made on the grounds that, while both models are proprietary in nature, the FORSYS model is better documented than its WEFA counterpart. A second consideration underlying this choice is that BMI currently has operational versions of the FORSYS model for France and Germany. Other models are planned for a number of major European economies, as well as the economies of Japan and Canada. Current national FORSYS models are linked by the BMI FORSYS-WORLDTRADE Accounting System. Hence, the entire modeling system could be used to examine both the direct and indirect impacts of $\mathrm{CO}_{2}$-induced environmental change on an international basis.

FORSYS is organized into five distinct blocks, as illustrated in Figure 7.1 . 


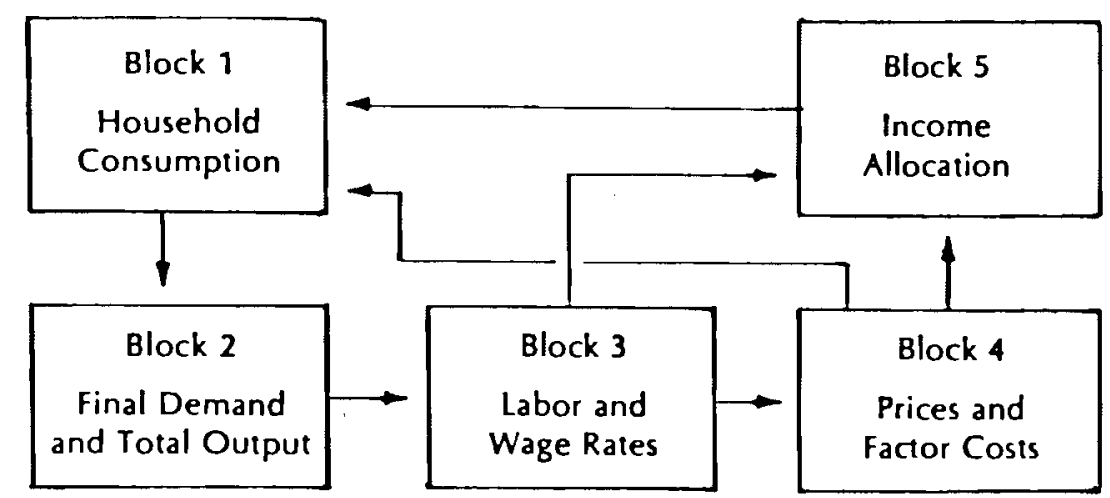

FIGURE 7.1. Schematic Diagram of FORSYS

Briefly, the functions of the blocks are as follows:

Block 1: Determine household consumption by commodity using prices and income.

Block 2: Solve for remaining components of final demand: investment, exports, imports, and government. Compute total output.

Block 3: Determine employment and wage rates.

Block 4: Use unit labor costs from Block 3 to determine prices. Compute gross output deflators (cost pass-through approach) and market supply prices. Compute profits (price-profit market tension or mark up on labor cost options).

Block 5: Aggregate wage and profit incomes. Compute disposable personal income. Send disposable income to Block 1 to close the model.

The solution process of the model requires the input of values of lagged endogenous variables, projected coefficients, and other exogenous variables. Key exogenous variables in the current model include total population, government spending, changes in stocks, import ratios (a function of the domestic market), exports, import prices, average hourly compensation and tax and subsidy rates.

To start the solution procedure the model requires initial estimates for total output and prices for each sector, and total disposable income. Given 
these estimates, the FORSYS program begins to work toward a solution by calculating initial estimates of imports, investment requirements, intermediate output, and private expenditure for each sector. The steps in this solution process are described below in terms of the blocks of the model. The model is solved by iteration within each year and produces annual forecasts of outputs from each block.

The U.S. model presently contains 59 consumer demand categories and 112 production sectors. Although large enough to provide adequate industry and consumption detail, a model of this size is still small enough to be efficient computationally. The solution algorithm for the model achieves this efficiency by relying heavily on "associated" data bases, organized along the same block definitions as the models. This allows independent block simulation of the mode1, making it easier to interpret the results of alternative policy changes or behavioral parameters. All of the industry and aggregate national results are stored on the data bases as they are estimated, and can be displayed in a variety of formats.

\section{Step 1: Consumer Demand}

Given initial estimates of prices and income, the model begins in Block 1 by calculating consumer demand by product. The supply prices by input-output sector are first used to construct implicit deflators of consumer goods categories. The price of these goods is constructed based on the output prices of the FORSYS manufacturing sectors that produce these goods, as we 11 as transportation and trade margins. A simultaneous system of demand functions is then employed to predict expenditures on 59 consumption categories. These functions, based on the indirect transcendental logarithmic utility function, allow for both substitution and complementarity relationships between final demand goods. The equations were estimated over the period 1955 to 1976 using data from the U.S. national accounts.

The consumer demand model is constructed as a two-step decision process, with consumers first allocating their budget among nine broad categories: food, fuel, clothing and footwear, household operations, household durables, private transportation, public transportation, health services, and other 
services and nondurables. After expenditures on each of these aggregate groups is determined, the model then allocates expenditures among the subcategories within each group. In this manner, consumer expenditures on each of 59 commodities is determined.

Step 2: Other Components of Final Demand

In Block 2, the other categories of final demand are estimated--exports, imports, government expenditures, inventory changes, and fixed investment.

Export and import forecasts are based on the BMI WORLDTRADE model. For a particular FORSYS simulation, exports are exogenous. Import forecasts are based on relative domestic market shares. Thus any policy change which increases domestic economic activity will also increase imports. Energy imports, such as crude petroleum and natural gas, are treated differently. Energy imports are determined as the projected residual between domestic demand and projected domestic supply; thus the domestic share changes with the level of economic activity.

Investment demands for producer durable goods, labor and energy are estimated for purchasing industries using input demand equations derived from a set of Generalized Leontief Cost Functions for each industry (Diewert 1971; Belzer and Roop 1981). As such, the demand for each of the these inputs by the jth producing industry is estimated econometrically as a function of industry output, the price of that input and the prices of the remaining inputs. Thus, substitution among inputs within each industry, as well as the distribution of a single input across industries, will be influenced by (a) input prices on the supply side of the economy, and (b) output prices on the demand side of the economy through the effect of changes in the prices of consumption goods upon industry output.

Investment by user for both equipment and structures is then converted to investment by product, using capital goods distribution matrices. These matrices are taken from projections of the Office of Economic Growth (Bureau of Labor Statistics, U.S. Department of Labor) from its long-term model of the U.S. economy. 
Step 3: Total Output

Once the vector of final demands by product is obtained $(F)$, gross outputs are computed by the familiar input-output relationship:

$$
X+A X=F
$$

where $A$ is the matrix of technical coefficients.

The A-matrices used in FORSYS are taken from the Office of Economic Growth, which forecasts the technical coefficients at five year intervals. Linear interpolation provides data for the intervening years. This initial set of technical coefficients is then surveyed by Battelle's research staff. This survey may suggest modifications to the projected coefficient matrices based on technological and engineering considerations derived from industry specialists. Thus, the matrix of technical coefficients used by FORSYS is based on the BLS growth model with modification provided by technical experts.

Step 4: Labor Demand and Wage Rates

Another of the derived factor demands from the Generalized Leontief Function is for the factor labor. This formulation predicts the labor output ratio as a function of relative factor prices. In forecasting, this estimating equation is transformed to predict man-hours by industry. The derived demand for labor function is similarly modified to allow for technological change and less than complete adjustment within any one time period.

FORSYS currently takes the aggregate wage level (including fringes and employer taxes) as exogenous. Relative wages by industry are explained by equations in which the principal explanatory variable is the unemployment rate and its lagged values. A price term captures the short-run effects of strong escalator clauses in the heavily unionized industries. Efforts are now underway to make wage determination endogenous within the model.

Step 5: Prices

The determination of domestic prices in FORSYS follows the price "dual" formulation of input-output theory. This formulation implies that material 
costs are "passed through" on a dollar-for-dollar basis. The components of "value added" per unit of output--labor compensation, profit-type income, and indirect business taxes--are treated separately:

- Unit labor costs are projected by multiplying the values of labor requirements per unit of output by the wage rate.

- Profit-type income is related to labor income as a function of a capacity measure, the wage/rental rate ratio, and a time trend. These equations are estimated at the 2-digit SIC level of aggregation for which wage and profit incomes are compiled by the U.S. Department of Commerce. The results of each regression at this level are then applied to all of the corresponding FORSYS sectors.

- Indirect business tax components are related to the most appropriate tax base that is available from the model. Ad valorem or specific excise taxes are related to either current or constant dollar shipments. Other taxes (primarily property taxes) are related to capital stocks by industry.

For some applications, an alternative method of estimating prices and profits may be advantageous. In the "price-profit minimization" procedure, the user supplies outside estimates of both future relative prices and profit margins for each sector. The sum of the "weighted" deviations of the model's prices and profit margins from those supplied by the user is minimized across all sectors via an optimization algorithm. This procedure might be used in cases in which the user assumes only a small amount of uncertainty regarding some future price or profit rate, but does not wish to impose that prior belief on the model unconditionally.

The final step in the cost computation is the calculation of domestic supply prices. Supply price is a weighted average of the price for domestic production determined through the procedure described above and imported commodities. The import prices are forecast exogenously, but the volume of goods imported is a function of the model. The following equation is used for calculating domestic supply price: 


$$
P_{S}=\phi\left(P_{m}\right)+(I-\phi) P
$$

where

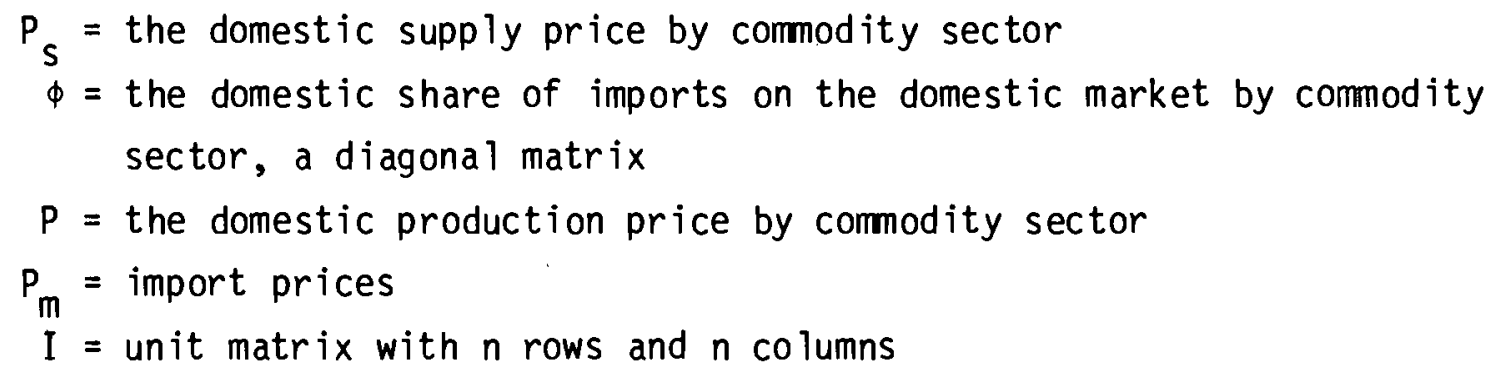

The supply price indexes, $P_{S}$, represent the price for commodity inputs for industrial purchase and the price to private consumers. The only additional requirement for private consumption is a converter matrix to modify commodity prices to spending-function prices.

\section{Step 6: Aggregate Incomes}

Block 5 of the model brings together the various price and quantity elements used to compute aggregate nominal incomes. Corporate profits and proprietor's income are estimated as functions of total property income. Dividends and transfer payments (exogenous in "real" terms) are the main additions to wage and proprietors' income in determining personal income. An average individual personal income tax rate is applied to obtain disposable personal income--(DPI) the major output of Block 5. With DPI in hand, model control returns to Block 1 . Blocks 1 through 5 are rerun until changes in prices, outputs, and disposable income all are small between consecutive iterations.

\section{Base Accounting System}

The FORSYS model uses a traditional national input-output accounting framework to describe the flow of intermediate goods through the economy. Thus, fairly rigid assumptions are maintained about the substitutability between raw materials and between raw materials and capital, labor and energy in production. It can be argued, however, that the additional expense of 
incorporating more flexible assumptions about these technological relationships for a great many industries is outweighed by the features that are added to the final demand and primary input sectors of the conventional input-output model. This is demonstrated by the fact that models which have used more flexible assumptions about the nature of interindustry demands for raw materials have generally contained a much smaller number of intermediate industries than are incorporated within the FORSYS interindustry accounting system (Hudson and Jorgenson 1974; Jorgenson and Fraumeni 1980).

\section{FORSYS Outputs}

The FORSYS model has the ability to produce numerous forecasts and to compare the results of selected forecasts. The following list provides a representative sample of forecasts available from the FORSYS model that are available for each of the commodity sectors, as well as the totals for all sectors.

- Macroeconomic demand components in current prices and constant 1972 prices, i.e., gross national product, private expenditure, government spending, productive investment in dwellings, imports and exports.

- Implicit nominal price indexes for GNP, domestic production, import, domestic market, and average annual growth rates for each of the sectoral price indexes.

- Macroeconomic cost components in current prices (employment income, gross profits, indirect taxes, and subsidies).

- Commercial balance of trade in current prices, i.e., the value of the difference between a country's imports and exports of goods and services.

- Sectoral values for domestic production, final demand components, and intermediate output, in both current and constant dollars.

- Growth and absolute values for labor, productivity, and wages. 
- Changes in investment requirements and capital-output ratios.

- Growth of exports, imports, and share of domestically produced goods at constant prices.

One of the advantages of using a model like FORSYS to project the indirect impacts associated with $\mathrm{CO}_{2}$-induced environmental changes is that a methodologically similar exercise has already been conducted using the Merrill-Lynch agricultural model (which is undocumented) in conjunction with FORSYS to forecast the indirect impacts of selected changes in agricultural policy on the national economy (Kite and Roop 1981). In this study, the effects of policy changes on agricultural commodity prices and output were simulated using the Merri1l-Lynch model. These price and output forecasts were then reaggregated in order to be consistent with the seven consumption and five input-output sectors that are contained in FORSYS. In the case of those seven commodities sold directly to final demand, the simulated price and quantity values obtained from the agricultural model were inserted into the appropriate demand functions as constants. The output levels of the five intermediate agricultural commodities were constrained at the point of sale to other industries in the economy, using the values from the agricultural mode1. The FORSYS model was then run to determine the indirect impact that these fixed prices and/or demands would have upon the national economy.

The same identical procedure could be employed in conjunction with estimates of $\mathrm{CO}_{2}$-induced agricultural commodity and price changes, without revision to the method described above. However, if the agricultural sector model used in the direct impact analysis phase of the research is a multi-regional model, then national values for commodity output and prices will have to be calculated from the regional values. There are many different ways of doing this and this does not represent a serious problem to the overall quality of the analysis.

\subsubsection{Normative General Equilibrium Models}

A number of authors (Manne et a1. 1978; Plessner 1957; Norton and Scandizzo 1981; and Yaron 1967) have investigated the conditions under which a 
linear programming model of an entire economy will provide solutions that are consistent with general equilibrium. While much of the research in this area has been focused upon theoretical considerations, the models that have been developed along these lines also have practical applications. Since there are a number of different ways of satisfying the necessary and sufficient conditions for an economy-wide, competitive general equilibrium solution within a linear programing framework, this section will focus on just one means of doing so. The approach that is presented in this section is from the work of Norton and Scandizzo (1981). It was singled out from all of the other models because it can be designed to incorporate information about the interindustry linkage structure of the economy from existing input-output tables and because of the authors' experience in constructing a small model of the Portugese economy based on the structure of this theoretical model.

The basic model can be written either as a nonlinear or linear programing model. Both versions are presented, not only to show the similarities between the two, but also because the development of new nonlinear programming algorithms (Murtagh and Sanders 1978) may permit the application of the nonlinear version of this model, provided that the number of final demand included in the model is fairly small.

The basic nonlinear model developed by Norton and Scandizzo includes $n$ goods $(i, j=1, \ldots, n)$, in $m$ resources $(k=1, \ldots, m), v$ consumers $(h=1, \ldots, v)$ and $z$ production technologies $(t=1, \ldots, 3)$ per good. However, to simplify the following analysis, it will be assumed that the preferences of all consumers can be characterized by an aggregate preference structure for a single, "representative" consumer (Gorman 1953, Muellbauer 1975, 1976). With this modification, the nonlinear model can be expressed as:

$$
\text { Maximize } Z=\sum_{i=1}^{n} P_{i} Q_{i}^{D}-\sum_{k=1}^{m} b_{k} r_{k}
$$

subject to 


$$
\begin{array}{ll}
Q_{i}^{D}-\sum_{t=1}^{z} Q_{i t}^{S} \leq 0 & \text { for all } i \\
\sum_{i=1}^{n} \sum_{t=1}^{z} d_{i k t} Q_{i t}^{S} \leq b_{k} & \text { for all } k \\
Q_{i}^{D}-\sum_{j=1}^{n} \psi_{i j} P_{j}-c_{i} M=a_{i} & \text { for all } i \\
\sum_{i=1}^{n} P_{i} Q_{i}^{D}-M \leq 0 & \\
P_{i}-\sum_{k=1}^{m} d_{i k t} r_{k} \leq 0 & \text { for all } i \text { and } t \\
Q_{i}^{D}, Q_{i}^{S}, R_{k}, M \geq 0 &
\end{array}
$$

where

$$
\begin{aligned}
P_{i}= & \text { the price of the } i \text { th good } \\
Q_{i}^{D}= & \text { the consumption of the ith good } \\
Q_{i t}^{S}= & \text { the output of the } i \text { th industry (sector) using the production tech- } \\
& \text { nology } t \\
r_{k}= & \text { the marginal value product (price) of the kth resource } \\
M= & \text { consumer income } \\
b_{k}= & \text { endowment of the kth resource } \\
d_{i k t}= & \text { the amount of the kth resource required to produce a unit of } Q_{j}^{S}, \\
& \text { using technology } t \\
\Psi i j= & \text { the change in the consumption of good } i \text { due to a one unit change in } \\
& \text { the price of } j \text { th good } \\
c_{i}= & \text { the change in the consumption of good } i \text { due to a one unit change in } \\
& \text { consumer income }
\end{aligned}
$$


$a_{i}=$ the maximum price that consumers are willing to pay to consume a single unit of the ith good

The objective function of this model is expressed in terms of the difference between personal consumption expenditures on all goods and total factor income. Under long-run competitive market conditions, the sum of these factor payments will exactly equal personal consumption expenditures. Thus, at the optimum, the value of $Z$ will be equal to zero. Equations (7.15a) represent a set of commodity balances, such that the consumption of good $i$ must be equal to, or less than, the total production of this good by means of the various technologies. Equation (7.15b) represents the resource constraints in the model. The shadow prices on these constraints will show the increase in marginal revenue associated with the production of the ith good due to a one unit increase in the availability of the kth resource. As this is the definition of the marginal value product of a resource, at an optimum the shadow price of the $k$ th resource will equal $r_{k}$. Both values are determined endogenous ly. Equation (7.15c) represents a series of linear, consumer demand functions for the $i$ th good. Linearity is assumed, here, only because it simplifies the transformation of this model into an LP model. In fact, any system of nonlinear demand equations may be used, provided that restrictions are imposed during the estimation of these parameters to ensure that preferences satisfy the axiom of convexity and other conditions to be addressed shortiy. Equation (7.15d) requires that personal consumption expenditures do not exceed consumer income. Finally, (7.15e) represents a set of marginal cost pricing conditions, which along with Equation (7.15b), directly implies constant returns to scale in production. This requirement is consistent with long-run competitive conditions.

The model expressed above can be explicitly altered to include interindustry transactions by rewriting the commodity balance $(7.15 \mathrm{a})$ and marginal cost pricing conditions ( $7.15 \mathrm{e})$, respectively, as

$$
Q_{i}^{D}-\sum_{j=1}^{n} \sum_{t=1}^{z} a_{i j t}^{\star} Q_{j t}^{S} \leq 0
$$




$$
a_{i i t}^{\star} p_{i}+\sum_{j=1}^{n} a_{j i t}^{\star} p_{j}-\sum_{k=1}^{m} d_{i k t} r_{k} \leq 0
$$

where each $a_{i j t}^{*}$ represents an element of the (I - A) Leontief matrix which has multiple technologies per good. Ordinarily, multiple technologies are not a feature of standard input-output models. Nevertheless, the inclusion of multiple technologies in the model provides a direct means of aggregating across sectors, thereby reducing the number of final consumption goods in the model $\left(Q_{j}^{D}\right)$ for which individual demand functions must be specified.

The above model contains two nonlinear features: in the objective function and in the constraint on personal consumption expenditures, where the expression $P_{i} Q_{j}^{D}$ appears in both. Short of using a nonlinear programming algorithm, there are two ways of eliminating this problem (although it is not clear that this does pose a problem, since newer nonlinear programming software may reduce the traditionally high costs of solving these problems to a level comparable with linear solution packages). The first approach would involve the use of quadratic programing to take care of nonlinearilities in the objective function, and then employing conventional separable programming methods to eliminate the nonlinearity in the personal consumption expenditure ronstraint. The second approach involves using the method of Duloy and Norton (1975), discussed in Section 6.2.2, to linearize both equations. The latter approach is the one adopted by Norton and Scandizzo (1981).

The modified objective function of such a model can be written as

$$
\text { Maximize } Z=\sum_{i=1}^{n} \sum_{s=0}^{s} \phi_{\text {is }} B_{i s}-\sum_{k=1}^{m} b_{k} r_{k}
$$

where, following the method of Duloy and Norton, $\phi_{\text {is }}$ is a parameter denoting the value of personal consumption expenditures on good $i$ at segment $s$ of the initial demand function, and $B_{\text {is }}$ is the variable weight on the demand function for good $i$ at segment $s$.

The segmented comodity balance constraints consistent with the linearization of (7.15a) are expressed as 


$$
\sum_{s=0}^{S} \theta_{i s} B_{i s}-\sum_{t=1}^{Z} Q_{i t}^{s} \leq 0 \quad \text { for all } i
$$

where $\theta$ is is a parameter denoting the quantity of good $i$ consumed at segment $s$ of the initial demand function.

The linearized demand functions consistent with (7.15c) are

$$
-\vec{Q}_{i 0}^{D} \sum_{s=0}^{s} \theta_{i s} B_{i s}+\sum_{j=1}^{n} n_{i j} \bar{P}_{j 0} P_{j}+E_{i} \bar{M}_{0} M=-1 \quad \text { for all } i
$$

where $\bar{Q}_{i 0}, \bar{P}_{j 0}$ and $\bar{M}_{0}$ represent the reciprocals of the initial values of $Q_{i}, P_{j}$ and $M$ (i.e., $1 / Q_{i 0}^{D}, 1 / P_{j 0}, 1 / M_{0}$ ), $\eta_{i j}$ is the price elasticity of demand for good $i$ with respect to the price of good $j$, and $E_{j}$ is the income elasticity of demand for good $i$.

The modified consumer expenditure constraint (7.15d) is

$$
\sum_{S=0}^{S} \phi_{\text {is }} B_{\text {is }}-M \leq 0
$$

Finally, the model must include a set of convex combination constraints which are

$$
\sum_{s=0}^{S} B_{i s}-E_{i} \bar{M}_{0} M-\sum_{i \neq j}^{n} \sum_{j}^{n} \eta_{i j} \bar{P}_{j 0} P_{j}=1+\eta_{i j} \quad \text { for all } i
$$

Thus, the final linearized version of the model can be expressed in terms of the maximization of a new, linearized objective function (7.16), subject to:

a) a linearized set of comodity balance constraints (7.17a),

b) the original resource balance constraints (7.15b),

c) a new set of linearized demand functions (7.17b), 
d) a linearized version of the household budget constraint (7.17c),

e) the original set of marginal cost pricing constraints (7.15e),

f) a set of linear, convex combination constraints (7.17d), and finally,

g) the usual nonnegativity restrictions on $B_{i s}, Q_{j}^{S}, P_{i}, M$, and $r_{k}$.

Two additional features of this model related to the linearization of the demand functions must be stressed. The first is that the price and income elasticity parameters in the model must be computed prior to running the model so that they satisfy certain specific conditions required by the theory of consumer demand. The theoretical meaning of these conditions are beyond the scope of this report; however, in the interests of precision they are:

(a) Engle aggregation, (b) homogeniety of degree zero in prices and income, and (c) Cournot aggregation. If constant elasticity demand functions are not utilized, then these conditions must be imposed on the basis of the initial values for $Q_{j}^{D}, P_{j}$ and $M$ which appear in the models as $\bar{Q}_{i 0}^{D}, \bar{P}_{j 0}$ and $M_{0}$, respectively. The second point is that these initial values must also be specified prior to running the model. This is not difficult to do and involves simply solving for $Q_{i 0}$, given an arbitrary vector of initial prices and income. However, this also means that if large exogenous stocks are introduced into the model, then one would be well advised to perform several interations, recalculating these initial values between iterations from the true (solution) values for $Q_{j}^{D}, P_{j}$ and $M$ obtained from the previous solution.

Introducing interindustry transactions into the linearized mode 1 requires an adjustment to the commodity balance constraints. This adjustment requires rewriting $(7.17 a)$ as

$$
\sum_{s=0}^{s} \theta_{i s} B_{i s}-\sum_{j=1}^{n} \sum_{t=1}^{z} a_{i j t}^{\star} Q_{j t}^{s} \leq 0
$$

The marginal cost pricing conditions are not affected by linearization of the model and the same adjustment defined for the nonlinear model, Equation (7.15e') can be substituted directly for (7.15e) in the linear version of the model. 
Either version of this model could be used to considerable advantage in assessing the indirect economic impacts associated with $\mathrm{CO}_{2}$-induced environmental changes. To do so would, however, require building such a model from the ground up. This would involve the following series of steps:

1. selection of an appropriate level of aggregation for the producing and final demand sectors of the economy;

2. appropriate modification of either the Department of Commerce or Bureau of Labor Statistics input-output table consistent with the aggregation scheme selected in step (1),

3. selection of fixed resources to include in the model and the development of technological coefficients for each of these resources, by producing sector,

4. estimation of the parameters of the consumer demand functions in the model, which may not be necessary if a choice is made to use a system of demands that have previously been estimated.

Once these steps have been carried out, either version of the model can be put in standard format using existing nonlinear or linear prograrming software. Following that, a period of debugging is ordinarily required to eliminate perverse results due to computational mistakes and/or incorrect parameter estimates.

Simulating the indirect impacts of $\mathrm{CO}_{2}$-induced environmental changes in the agricultural sector could then be accomplished by utilizing information from the direct impact analysis in the following manner. Each $Q_{i t}^{S}$ within the mathematical programming model represents the level of production for industry $i$ using technology $t$. Some set of these output variables will be agricultural cormodities. Values for those commodities that are projected during the primary impact analysis can be inserted as exogenous, fixed values into the normative model. Given these values, the normative model will allocate available resources in a manner that will be consistent with the objective function. The results obtained from such an exercise will provide optimal values for the remaining, constrained activities in the model. This output will consist of values for: 
1. production of unconstrained (nonagricultural) industries;

2. consumption of final goods and services for those commodities whose final demands are not constrained;

3. equilibrium prices for final demand goods;

4. personal consumption expenditures; and

5. prices for primary resources included in the model.

Based on these estimates, additional values can be calculated for:

6. gross national product;

7. gross income originating, by sector;

8. value added, by sector; and

9. various measures of consumer welfare.

Thus, this approach represents a potentially very powerful tool in the analysis of the indirect economic impacts due to increases in ambient $\mathrm{CO}_{2}$. The advantages of this type of model over input-output models are that (a) it is "driven" by a set of consumer demands, rather than assuming that demands are exogenous, (b) consumption levels, output and prices are simultaneously determined, and (c) these advantages are achieved without sacrificing the detailed (if still somewhat simplistic) characterization of production technology that is embedded within conventional input-output models. The advantages of this type of model over one like FORSYS are, first, that it is possible to include a much larger number of primary resources, and secondly that the equilibrium prices for all primary resources are determined endogenously. On the other hand, normative models are not dynamic, whereas FORSYS is. Another disadvantage to this approach is that no known model, similar in structure to the ones described above, currently exists for the U.S. marcoeconomy. Thus, the potential benefits associated with building and using such a model must be weighed against its costs and the alternatives provided by other models. 


\subsubsection{Regional Econometric Models}

All of the models discussed in previous sections of this chapter are macro models of the U.S. economy. However, both of the models recommended for use in the direct impact analysis phase of this research, AGSIM and the CARD Tatonnement (CT) model, are multi-regional in character. Therefore, it might also be desirable to use a linked multi-regional model in the indirect impact analys is phase of this research in order to take advantage of the regional detail in either AGSIM or the CT model. It should be stressed, however, that while the use of multi-regional models in both phases of the analysis will help to reduce the aggregation problem, none of the regional econometric models examined in this phase of the research is a perfect mate to either AGSIM or the CT model in terms of the ir respective multi-regional delineations. As a result, results that are obtained either from AGSIM or the CT model in the first phase of the analysis will have to be re-aggregated in order to be consistent with any regional econometric model used in the second phase.

Several relevant multi-regional economic models were reviewed. These included the National Regional Impact Evaluation System (NRIES) model (Ballard and Gustley 1980), the multi-regional multi-industry (MRMI) model (Harris 1980), the Oak Ridge National Laboratory/Charles River Associates (ORNL/CRA) model (0lsen et al. 1977; 0lsen et al. 1980), the Wharton Econometric Forecasting Associates (WEFA) model (From et al. 1979) and the Pacific Northwest Laboratory (PNL) MASTER model. A detailed discussion of each of these models is beyond the scope of this report. However, since most regional econometric models appear to be related in concept and structure to a common origin (Klein 1969; Glickman 1974; Klein and Glickman 1977) it is possible to describe the major features of most of these models in general terms. A possible exception to this last statement is the PNL MASTER model. This model contains some features that are substantialiy different than those in the other models reviewed. Therefore, following the general discussion, a brief overview of the MASTER model will be provided.

(a) Scott, M. J. et al. 1981 (Draft). The Metropolitan and State Economic Regions (MASTER) Model. Pac ific Northwest Laboratory, Richland, Washington. 
Most multi-regional econometric models are designed around the distinction between basic industries which produce goods and services for export and nonbasic, or domestic industries, which produce goods and services that are consumed within the region. However, a basic industry in one region is not always a basic industry in another region. For that reason, the demand for any regionally produced good should theoretically be divided into basic and nonbasic components. Whether this can be done will depend on the availability of data to make such a distinction. If not, then the only other resort is to identify which industries are basic and which industries are nonbasic and estimate different demand equations for the two sets. Consistent with either of these two formulations, the demand for basic goods and services can be expressed as

$$
Q_{i j}^{B}=f_{i j}^{B}\left(P_{j} / P_{u s}, D_{u s}, T C_{i}\right)
$$

while the demand for nonbasic goods is expressed as

$$
Q_{i j}^{N B}=f_{i j}^{N B}\left(P_{j} / P_{u s}, D_{j}\right)
$$

where $Q_{i j}$ represents the output of industry $i$ in region $j$, the superscripts $B$ and $N B$ denote basic and nonbasic production, $P_{j} / P_{\text {us }}$ is a vector normalized prices (regional price divided by national, U.S. price) for good $i$ as well as other goods that are either substitutes for or complements to the reference good, and $\mathrm{TC}_{i}$ is the transportation cost of good $i$. The variable $D$ is a demand-driving variable which is referenced to national forces, in the case of basic goods, and to local forces in the case of nonbasic goods. At the national level, $D_{\text {us }}$ could be GNP; it could be total population; or it could be national output for good $i$. For nonbasic goods, $D_{j}$ could be gross regional output (GRP), regional population or income, or regional output of industry $i$. 
Regional supply functions for industrial output are usually not distinguished along basic and nonbasic lines. These supply functions for good i can be expressed generally as

$$
Q_{i j}^{S}=g_{i j}\left(P_{j} / P_{u s}, w_{i j}, r_{i j}, K_{i j}\right)
$$

where $w_{i j}$ represents the wage rate for industry $i$ in region $j, r_{i j}$ is a vector of industry input prices for energy, capital and purchased materials, and $k_{i j}$ is the capital stock for industry $i$ in region $j$ during the current period. Only a single input price variable, the wage rate, is included in Equation (7.19); however, depending on the availability of data (which is a function of the level of regional aggregation) and the industry other input costs can be included in the regional supply functions. Notice also that Equation (7.19) does not contain any reference to the period in which explanatory variables are measured. In some cases, regional supply functions include arguments in lagged output prices and output. This is consistent with the application of stock adjustment and adaptive expections models to regional supply relations. However, some critics of regional econometric models have argued that treating industry output in a recursive fashion is done primarily to eliminate the degrees-of-freedom problem associated with estimating Equation (7.18) and (7.19) simultaneously (Richardson 1973). If the recursive framework is adopted, such that a lagged dependent variable is included in Equation (7.19), then the output of the ith industry will be predetermined up to the point of demand as is the case with most econometric models of the agricultural sector.

The structure of the market clearing mechanism will depend on the treatment of demand. If the demand for good $i$ is divided into basic and nonbasic components then the market clearing identity will be

$$
Q_{i j}^{B}+Q_{i j}^{N B}-Q_{i j}^{S}=0
$$


On the other hand, if basic and nonbasic industries are different, then for basic industries the market clearing identity will be

$$
\left.Q_{i j}^{B}-Q_{i j}^{S}=0 \quad \text { (BASIC: } \quad \text { i } E B\right)
$$

and for nonbasic industries, it will be

$$
\left.Q_{i j}^{N B}-Q_{i j}^{S}=0 \quad \text { (NONBASIC: } \quad i E N B\right)
$$

The supply function shown in Equation (7.19) contains an argument in $k_{i j}$, a capital stock term. In the short-run, $k_{i j}$ is fixed. However in the long run, the capital stock in an industry is variable due to new investment. The treatment of investment varies considerably across different models. However, most of the methods that are employed represent more or less sophisticated variations on the principles that underly the standard, stock adjustment model. For example, in the PNL MASTER Model the desired level of capital stock in industry $i$, in region $i$ during period $t$ is expressed as

$$
K_{i j t}^{\star}=n_{i j}\left(U C_{i j t}, w_{i j t}, Q_{i j t}\right)
$$

where UC is the user cost, or price, of capital, $w$ is the wage rate and $Q$ is output (the superscripts have been dropped for convenience). New investment is defined, using a stock adjustment model (see Section 6.2.1 for further explanation), as

$$
k_{i j t}-k_{i j t-1}=(1-\lambda)\left(K_{i j t}^{\star}-k_{i j t-1}\right)
$$

Replacement investment is assumed to be proportional to the capital stock in the previous period, or 


$$
\delta K_{i j t-1}
$$

Substituting Equation (7.22b) into (7.22a) for $k_{i j t}^{\star}$ gives a solution for the actual capital stock as

$$
k_{i j t}=(1-\lambda) h_{i j t}\left(U C_{i j t}, w_{i j t}, Q_{j t}\right)+\lambda K_{i j t-1}
$$

Total investment, which is the sum of net and replacement investment, then becomes

$$
I_{i j t}=(1-\lambda) h_{i j t}\left(U C_{i j t}, w_{i j t}, Q_{j t}\right)+(\delta+\lambda) k_{i j t-1}
$$

With the exception of the ORNL/CRA model, all of the remaining models reviewed contain features that permit them to forecast regional labor force, employment, unemployment and wage rates. Technical difficulties usually arise in specifying the equations in this part of a multi-regional model because (a) the demand for labor is not observable in the sense that employment does not equal the demand for labor (due to unemployment), and (b) unemployment (which is the difference between supply and demand) is not observable on an industry-by-industry basis. As a result, simultaneous estimation of the supply and demand for labor is not possible. Therefore, concessions are usually made to theory, either by assuming that unemployment is voluntary or by recasting the labor demand components into employment demand functions. The PNL MASTER model uses the latter approach. In this model, it is assumed implicitly that the quantity of labor demanded represents the number of people working. Employment demands $\left(L_{i j}\right)$ are

$$
L_{i j}=L_{i j}\left(P_{i j}, w_{i j}, r_{i j}\right)
$$

where $P_{i j}$ is the price of industry i's output in the $j$ th region, $w_{i j}$ is that industry's wage rate and $r_{i j}$ is a vector of industry input prices for energy, capital and purchased materials. 
Labor supply in MASTER is formulated in terms of regional labor force $\left(L F_{j}\right.$ ) equations, which can be expressed as

$$
L F_{j}=L F_{j}\left(\hat{w}_{i j}, P O P_{j}\right)
$$

where POP $j$ is regional population, and $\hat{w}_{i j}$ is the normalized, employment-weighted average wage rate, or

$$
\hat{w}_{i j}=\sum_{i=1}^{n} w_{i j} L_{i j} /\left(C P I_{j} \sum_{i=1}^{n} L_{i j}\right)
$$

Total labor force is the sum of industrial employment demands plus unemployment $\left(U_{j}\right)$, or

$$
L F_{j}=\sum_{j=1}^{n} L_{i j}+U_{j}
$$

However, the wage rate, $w_{i j}$, is assumed to be an equilibrium wage only if the unemployment rate or real wages do not change. Actual, subsequent changes in real wages in each industry will then be caused by the overall loosening or tightening of the unemployment rate and by increases or changes in the mix of employment. This effect is captured by a real wage rate equation

$$
\Delta \frac{W_{i j}}{C P I_{j}}=\phi\left[\Delta\left(U_{j} / L F_{j}\right), \Delta L_{i j}\right]
$$

This procedure allows for differing occupational skills by permitting the mix of industrial growth to affect wage rate changes in a region. Presumably, a given industry's average wage will be affected more profoundly by another industry which grows very quickly and uses the same mix of occupations than it will be by an industry which grows more slowly or which uses a markedly different mix of skills (Richardson 1973). None of the other models surveyed 
make allowances for this type of phenomenon which has historically played a fairly dynamic role in U.S. urban and regional economic development (Pred $1966)$.

With the exception of the ORNL/CRA model, all of the remaining models reviewed predict income, broken down by various categories. A comprehensive disaggregation of income would include the following components:

- wage and salary income

- total labor income

- farm income

- transfer income

- dividends, interest and rental income

- labor and proprietor income

- personal income

The conventions for estimating the endogenous components of income are beyond the scope of this report. However, of the models surveyed, only the MRMI and MASTER models include provisions to calculate all of the above components.

Finally, most of the models reviewed contain a fairly detailed demographic section to predict regional population, births, deaths and migration. The major differences among these models relate to (a) the components of population change which are treated as exogenous to the system, (b) whether migration is calculated on a net or gross basis, and (c) whether population is disaggregated by age-sex groups. Under ideal circumstances, the regional population would be decomposed into birth cohorts, by sex. Births would be determined endogenously as a function of the number of women of child bearing age, the sex ratio for selected age classes, wages, female employment and hourly earnings and employment rates by age group. Separate equations would be used to predict in- and out-migration. Only deaths would be exogenous. In concept, none of the models contain this degree of sophistication. However, both the WEFA and MASTER models approach population from the perspective of disaggregated birth cohorts. In that regard, the WEFA model also disaggregates employment by twelve specific age-sex groups, whereas 
MASTER calculates total labor force. None of the models reviewed estimate both in- and out-migration from a region, largely it appears because of data problems. Finally, none of the models, save MASTER, treats all of the components of population change (except deaths) as endogenous.

In concept, PNL's MASTER model contains a number of desirable features which are not usually built into other multi-regional models. First of all, MASTER is disaggregated on a Standard Metropolitan Statistical Area (SMSA), rest-of-state basis. Only the MRMI county-level model contains a larger number of regions. However, SMSA data bases are generally far richer in industrial and demographic detail than are county-level data. A second feature of the MASTER model that sets it somewhat apart from the rest is the consistency of many of its structural equations with the neoclassical theory of the firm. Either because of tradition or else because of the size of the basic regional unit employed, most multi-regional econometric models have leaned heavily on macroeconomic theory in the specification of structural equations. In MASTER, on the other hand, the regions are small enough to assume that firms behave as price takers in competitive markets. This makes it possible to derive industry supply and input demand functions in each region that are consistent with the profit maximizing behavior of a multi-output, multi-input firm. There are numerous advantages of doing this, not the least of which are (a) it reduces the need for trial and error, ad hoc specifications for many model equations, and (b) it permits the theoretical imposition of certain restrictions on the parameters of the supply and input demand equations which, in other applications, tend to make econometric models more stable, and to prevent perverse results in model forecasts.

The MASTER model is also fairly unique in that is contains an industrial location component that is present only in the MRMI model. The formulation contained in the model is due to Aukrust (1970) who suggested that locational forces which determine the geographic redistribution of economic activity could be modeled in a choice theoretic framework. Building on this work, industries in MASTER are categorized as "exposed" or "sheltered" from economic stimuli that are external to the region. For exposed industries, the effect 
of locational influences is captured by estimating a series of "logit" equations in which the national share of industry i's output in region $j$ is a function of regional wage rates, energy and material prices, the user cost of capital, government expenditures, taxes and an industrial collocation variable. For sheltered industries, the ratio of regional output in each industry to regional population is estimated in a logit framework as a function of regional income, population, wage rates and energy prices, taxes, government expenditures (including transfer payments) and a sheltered industry collocation variable.

A final feature of MASTER that sets is apart from other multi-regional models lies its specification of the construction sector. In many regions, the construction industry plays an important role in the domestic economy. Historically, the construction industry has proven especially sensitive to national business cycles. In those regions where construction activity has figured significantly in terms of its contribution to gross regional product, the cyclical behavior of the construction industry has often had very profound impacts on the regional economy. Unfortunately, efforts to predict the behavior of this industry have not proven very successful. Moreover, most models of the construction industry have tended to focus on the residential construction sector at the expense of the cormercial sector, whereas in MASTER both sectors are highly disaggregated. However, the real strength of the MASTER model's construction sector appears to lie in the unique theoretical approach used to capture the behavior of economic agents in these markets. For example, in both the residential and commercial buildings sectors, both the flow of building services (viewed as a consumption good) and the stock of buildings (viewed as an asset) are modeled jointly. Market clearing processes are identified for both the flow and asset markets, but market clearing behavior in one market is jointly determined by behavior in the other market. This is not only an extremely realistic way of modeling the two buildings sectors; it also appears to provide very accurate forecasts of the turning points in the cyclical behavior that is so common to the construction sector as a whole. The joint market clearing model was recently applied to 
forecasting commercial floor space estimates for 10 different commercial buildings categories in the Pacific Northwest. (a) The performance of the model was excellent.

Using a multi-regional model to predict the disaggregated, indirect impacts of $\mathrm{CO}_{2}$-induced environmental changes in the agricultural sector would not be a methodologically difficult exercise. In order to provide a better understanding of how this might be done with a specific model, the following discussion will focus on the use of the MASTER model in the indirect impact analysis phase of the research.

Figure 7.2 is a schematic illustration of the MASTER model. For each of the 273 SMSAs and 48 rest-of-state regions in MASTER, there are essentially five distinct submodels. They are:

- An Industrial Location Submodel--which determines regional output for exposed and sheltered industries at the 1-digit Standard Industrial Classification (SIC) level. (A large-scale model will be estimated for 2- and 3-digit level SIC industries during FY-82.)

- An Investment Determination Submodel--which calculates net and gross investment during each period and supplies new capital stock estimates to the Industrial Location submodel to be used in projecting the next period's output.

- A Labor Market Submode 1--which determines employment and wage rates by industry, as well as regional labor force and unemployment.

- A Personal Income Determination Submodel--which uses information on employment and wage rates to calculate various components of personal income.

- A Population Determination Submodel--which uses information on employment, wage rates, personal income and deaths to calculate births, net migration and total population.

(a) Staloff, S. J. and R. C. Adams. 1982 (Draft). The Development of a BPA Region Commercial Floor Space Model. Pacific Northwest Laboratory, Richland, Washington. 
MASTER MODEL

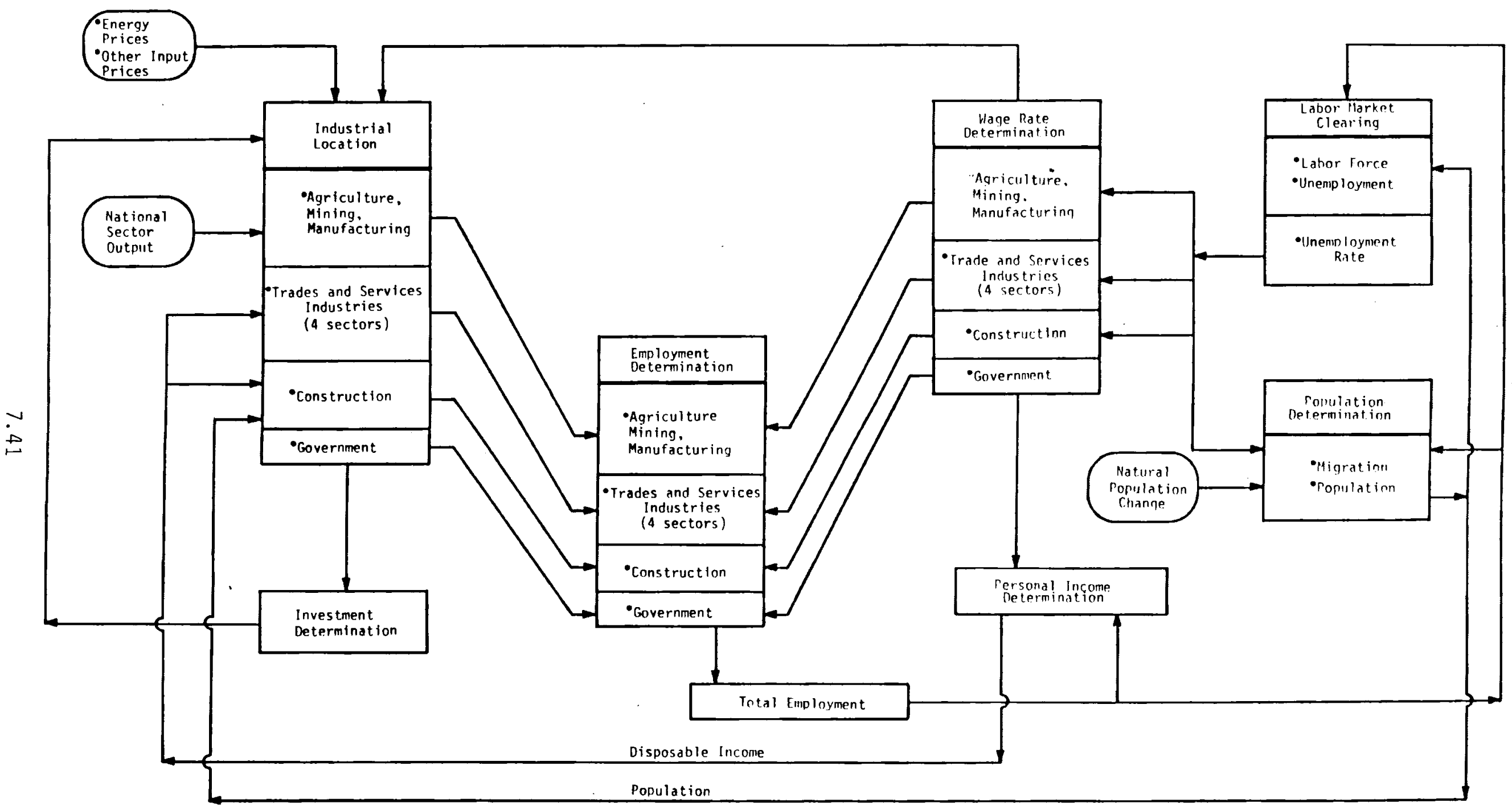

FIGURE 7.2. Schematic Overview of MASTER Model 
Exogenous inputs to MASTER in standard simulation runs include:

- U.S. output by industry

- U.S. wages by industry

- prices for nonlabor factors of production

- CPI

- U.S. interest rate/regional interest rates

- existing capital stock (in the initial period)

- death rates by age-sex groups

Annual outputs from the standard simulation run include:

- output supplied by industry

- wages by industry

- employment by industry

- unemployment

- labor force

- labor income

- nonfarm proprietors' income

- farm income

- transfers

- dividends, interest, rents

- disposable income

- net migration

- natural population change

- population

- manufacturing nonconstruction investment

The indirect impacts of $\mathrm{CO}_{2}$-induced environmental changes on these model outputs could be accomplished in a relatively simple manner. Estimates of agricultural output from the direct impact analysis phase of the research would first have to be aggregated up to either the 1-digit or 2-digit SIC leve1. Once this was accomplished, one of two procedures could be followed. The first alternative would involve using a macro-model to supply the necessary values of national output for all industries, including the agricultural sector and inserting these fixed values into the Industrial Location submodel as a part of the National Sector Output vector shown in the 
upper left-hand corner of Figure 7.2. The other alternative would consist of disaggregating agricultural output to a level that is consistent with the SMSA, rest-of-state regional delineation contained within the model. Regional agricultural output would then be held constant in the Industrial Location submodel. Using this latter approach, regional agricultural output would be forced to be consistent with the estimates obtained from the direct impact analysis phase of the research. In the former approach, national agricultural output would be held constant and agricultural output would be redistributed on a regional basis.

The distinction between these two alternatives is highly significant. It means that, if one does choose to select a national agricultural sector model to conduct the direct impact analysis, then MASTER could be used to redistribute both the direct and indirect impacts of changes in agricultural output at the national level among the regions contained in this model for all industries, including agriculture. However, this would not eliminate the need to use a macro-model of the national economy in order to obtain national values for other exogenous variables required to drive MASTER.

\subsection{INDIRECT IMPACTS ON THE ALLOCATION OF PRIMARY RESOURCES}

In the previous section, most of the emphasis was placed on examining different types of models which can provide information about the indirect economic impacts of $\mathrm{CO}_{2}$ on the production and consumption of intermediate and final goods. However, if $\mathrm{CO}_{2}$-induced environmental changes are substantial enough, then fairly profound impacts may occur in terms of the redistribution of primary inputs, or fixed resources, between different sectors of the economy. Without indulging in too much idle speculation, it is still possible to suggest that if such reallocations due occur, the ones that will be of greatest interest to policy makers and firms in the private sector involve land and water resources.

As a general rule, econometric agricultural sector models take a short-run minded approach to land. The acreage planted in any given year is treated, at least in part, as a function of last year's planted acreage. Thus, the possibility that some of last year's cropland has been turned into 
condominiums cannot be entertained within the structure of these models. Water generally receives less treatment. In none of the econometric models surveyed for this report was there a single model in which either the price of irrigation water or the availability of that resource was included as a dependent variable in the acreage response or yield equations. This does not mean that there are no econometric models which focus on water resource use by the agricultural sector. There are, but these models (Gardner and Fullerton 1968; Frank and Beattie 1979) are highly specialized and do not qualify as agricultural sector models.

Normative models, like the Iowa State CARD linear programing model discussed in Section 6.2.2, focus directly on those two resources. However, the interaction between agricultural and nonagricultural land and water uses can only be viewed in a partial equilibrium framework, by changing the prices of these two resources exogenously. In point of fact, of course, the value of both land and water resources at the margin will be determined jointly through the competition between agricultural and nonagricultural uses for these resources. The remainder of this section will examine different approaches that can be used to evaluate $\mathrm{CO}_{2}$-induced changes in the interindustry distribution of 1 and and water resources.

\subsubsection{Resource-Based Input-0utput Models}

A number of researchers have used input-output techniques to determine the required levels or impacts on factor inputs such as employment (Isard and Kuenne 1953; Kutscher and Bowman 1974; Moore and Petersen 1955) and energy flowing from changes in final demands (Bullard and Herendeen 1975a; 1975b; Herendeen 1973; Wright 1975). In addition, input-output methods have been adapted for projecting the water resource requirements associated with given levels of final demand (Kelso, et al. 1973; Lewis et al. 1973; Lofting and Davis 1968; Lofting and McGauhey 1963; Miernyk 1969; Tijoriwala et al. 1968). This section examines how such techniques can be applied to the issue of water and land resource requirements in general, and to water resource-related secondary impacts due specifically to $\mathrm{CO}_{2}$-induced environmental changes. 
To construct a resource content matrix, it is first necessary to develop resource requirements coefficients for each resource and sector. A resource requirements coefficient is calculated by dividing the total amount of the resource used by a sector by the gross output of the sector. Focusing on a single resource, and denoting the quantity used by sector $i$ as $R_{i}$, allows one to write the industry requirements coefficient as

$$
b_{i}=\frac{R_{i}}{x_{i}} \quad i=1, \ldots, n
$$

where, as before, $x_{i}$ is the gross output of industry $i$. The resource contents matrix, $w$, can be calculated by premultiplying a diagonal matrix of the $b_{j}$ by the transpose of the Leontief inverse matrix $(I-A)^{-1}=Q$, or

$$
R=Q^{\prime}\left\langle b_{i}\right\rangle=\left[r_{i j}\right\rfloor \quad i, j=1, \ldots, n
$$

where $Q^{\prime}$ denotes the transpose of $Q$ and $<$ represents a diagonal matrix. Each entry, $r_{i j}$, represents the resource content (measured in physical units) of the output of industry $j$ required by industry $i$ for the latter industry to deliver a dollar's worth of output to final demand. Row sums in $R$, or

$$
r_{i}=\sum_{j=1}^{n} r_{i j}
$$

form a vector of direct and indirect resource requirements for each dollar's worth of output by industry $i$.

With this information, one can then use input-output techniques to analyze the feasibility of given final demands with respect to resource requirements. For example, by determining the necessary level of gross output, $\hat{x}$, to meet a specified vector final demands, $\hat{y}$, resources requirements for each industry are given by 


$$
\hat{R}=\left\langle r_{i}\right\rangle \hat{x}
$$

where $r_{i}$ is the direct and indirect requirements vector from Equation (7.26). If the supply of the resource is less than those projected to meet $\hat{x}$, i.e. $R<\hat{R}$, final demands must be adjusted downward.

Given the structure of the standard input-output model it would, appear that while sensitivity analysis of resource-related secondary impacts due to $\mathrm{CO}_{2}$-induced environmental changes could be undertaken, potential decreases in the supply of the resource or the efficiency of resource use that might be caused by these changes cannot be examined directly. All that can be done in this framework is to examine the feasibility of meeting different levels of final demand with different resource supplies. As such, this approach is clearly inadequate because, a priori, final demands will be unknown.

One way of introducing implicit price competition between sectors in the input-output framework is to embed the Leontief matrix within an optimization mode1. For example, Lofting and McGauhey (1963) have employed the following linear programming model to analyze different water supply scenarios on the interindustry distribution of water resources in the state of California.

$$
\operatorname{Maximize} z=\sum_{j=1}^{n} v_{j} x_{j}
$$

subject to

$$
\begin{aligned}
& \sum_{j=1}^{n} b_{j} x_{j} \leq w \\
& \sum_{j=1}^{n} a_{i j}^{\star} x_{j} \geq Y_{i} \quad \text { for all } i=1, \ldots, n \\
& x_{i}>0
\end{aligned}
$$


where

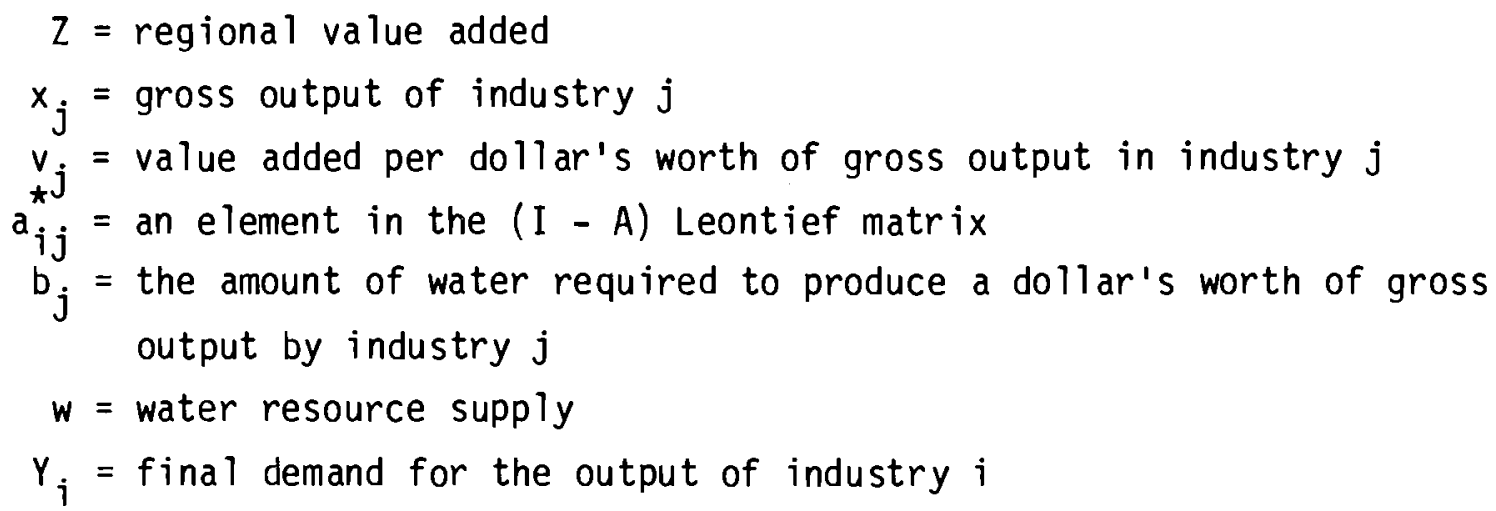

The objective function of this model is stated in terms of maximizing value added. This objective is consistent with competitive conditions in factor markets. Equation (7.29a) is a water resource commodity balance, and Equation (7.29c) can be written in more familiar terms as ( I - A) X $\geq Y$. Within this framework, one could analyze the secondary impacts of $\mathrm{CO}_{2}$-induced environmental changes on water resources (or land, by simply recasting the commodity balances to reflect land use and availability) by: (1) varying the $b_{j}$ in the agricultural sector to reflect changes in resource use efficiencies, and (2) by varying the supply of the resource, $w$, and comparing the pre- and post-change values of $Z$. The secondary impacts would then be, simply,

$$
z_{1}-z_{0}-\left(v_{a 1} x_{a 1}-v_{a o} x_{a 0}\right)
$$

where $v_{a}$ and $x_{a}$ are the value added and output from the agricultural sector and the subscripts 0,1 denote pre- and post-change values, respectively. While prices plays an active role in this model in determining how water is allocated, the major drawback of this approach is that final demands are still exogenous. Hence, this type of model cannot reflect simultaneous adjustments that consumers might make in their purchases due to changes in the cost of the goods they purchase as a result of higher or lower resource prices.

The results of the Lofting-McGauhey study are nevertheless illustrative of the potential sensitivity of the existing interindustry distribution of 
water in California to possible $\mathrm{CO}_{2}$-induced changes in the availability of that resource. All estimates of the shadow price of water (the change in $Z$ due to a one unit increase in the supply of water) are in 1959 dollars per acre foot. For a feasible solution, approximately 43 million acre-feet are required; at this level of availability, the shadow price of water is $\$ 11,260$. As the constraint is lessened, price falls rapidly (e.g., within a range of 43 to $48 \mathrm{million}$ acre-feet the price is $\$ 5710$; within 49 to 51.5 , $\$ 1160$; within 51.5 to $51.8, \$ 790$; within 51.5 to $66.7, \$ 450$; and, within 66.7 to $78.9, \$ 150)$. It is quite clear that, at low levels of availability, the value (i.e., shadow price) of water is quite high and only those activities in which the marginal value of water is also quite high would be able to "compete" for water.

While the application of traditional demand-oriented input-output analysis appears to have limited usefulness in analyzing the secondary water-resource impacts of $\mathrm{CO}_{2}$-induced environmental changes, a variant of the traditional analysis may be more promising. An input-output table is a neutual image of an economy, emphasizing neither demand nor supply applications. While the vast majority of input-output applications have emphasized the backward linkages from final demand to gross output to primary inputs |e.g., see Equation (7.33a)|, applications could also be based on forward linkages (i.e., from primary inputs to gross output to final demand). Ghosh (1958) has formulated an input-output application based on an allocative structure. This allocative structure has also been discussed by Augustinovics (1970) and applied by Giarratani (1976) to the issue of energy shortages.

This approach is specified below.

The basic identities underlying input-output are

$$
\begin{aligned}
& x_{i}=x(1)+y \\
& x_{i}=x^{\prime}(1)+v
\end{aligned}
$$

Usually, the input-output table is transformed into an analytical model by substituting the linear-demand relationship, 


$$
x_{i j}=a_{i j} x_{j} \quad i, j=1, \ldots, n
$$

into identity (7.30a) and solving gross output as a function of final use. Following Ghosh (1958), however, one may also assume

$$
x_{i j}=\bar{a}_{i j} x_{i}
$$

That is, interindustry flows from industry $i$ to $j, x_{i j}$, are a proportional function of $i$ 's output $\left(\bar{a}_{i j}\right)$ and not a proportional function of $j$ 's output $\left(a_{i j}\right)$ as in Equation (7.31).

Taking the transpose of (7.30b) and converting (7.32) to matrix notation, one obtains

$$
x^{\prime}=(1)^{\prime} x+v^{\prime}
$$

and

$$
\text { (1) } x=x \cdot \bar{A}
$$

where $\bar{A}=\left[\bar{a}_{i j}\right]$ are the output coefficients which indicate direct sales from $i$ to $j$ per unit of output of sector $i$. Given (7.33b), the supply identity may be solved for gross output in terms of primary inputs and structural parameters

$$
x^{\prime}=V^{\prime}(I-\bar{A})^{-1}
$$

or

$$
x^{\prime}=v^{\prime} \bar{Q}
$$


where $Q=(I-\bar{A})^{-1}$. Each element in $\bar{Q}, \bar{q}_{i j}$, is a supply multiplier, relating unit changes in primary inputs to changes in gross output

$$
\bar{q}_{i j}=\partial x_{j} / \partial v_{i}
$$

Equation (7.35) implies that changes in the level of primary inputs to industry $i$ permit changes in its level of output, as well as in the levels of output of sectors requiring its output for their own use. Both the direct and indirect effects of this activity are reflected in $\bar{q}_{i j}$. Given exogenous inputs, the formulation of the allocative structure implies that demand is always forthcoming to meet increased output, the reverse of the usual formulation.

Row sums of the matrix $\bar{Q}$, or

$$
q_{i}=\sum_{j=1}^{n} q_{i j}
$$

are multipliers describing the total output in the economy per unit increase in primary inputs of sector $i$ (i.e., the total direct and indirect economy-wide impacts); therefore, $q_{j}-1$ gives the total economy-wide indirect effects. In fact, Giarratani (1981) has computed both $\bar{q}_{j}$ and $\bar{q}_{j}$ for all sectors based on the United States interindustry table for 1967 (BEA 1967). Results for selected industries are shown in Table 7.2.

As can be seen, the indirect effect of a one unit change in primary supply to agricultural products is 2.61 . Of course, one could also calculate the final use multiplier (i.e., the change in final use of industry $j$ induced by a one unit change in primary supply to $i)$.

And, as Giarratani has shown, the model can be applied to the problem of allocating scarce resources to both intermediate and final use. Given $x^{\prime}=$ $v^{\prime} \bar{Q}$, one can examine the impact of alternative allocations of primary inputs 
$\mathrm{CO}_{2}$-induced environmental changes on the distribution of water and land resources, but one that is still not appropriate for the current research problem.

\subsubsection{Normative Mode 1s}

Given the limitations of input-output techniques, this section will focus briefly on the use of normative models to evaluate the indirect impacts of $\mathrm{CO}_{2}$-induced environmental changes on the distribution of water and land resources among competing industries.

First of all, it would be possible to use the CARD Tatonnement (CT) model to conduct a partial equilibrium analys is of these impacts. In the current version of the model, urban and other nonagricultural land use demands are dealt with exogenously. Based on current estimates of the future demand for land from nonagricultural industries in the year 2030, approximately 48 million acres of land have been removed from the agricultural land base. However, it would not be difficult to restructure the model such that future transfers of land from agricultural to nonagricultural uses would take place within the model based on a partial, competitive equilibrium in the market for this resource. This could be accomplished by adding a land transfer activity to each production area which would allocate land from the current agricultural land base to either the agricultural sector or to another exogenous, nonagricultural sector. Each of these transfer activities would comprise an additional row in the constraint matrix of the model in the form

$$
A_{1 r}+A_{2 r} \leq A_{0}
$$

where $A$ is acreage, the subscripts 1 and 2 denote agricultural and nonagricultural uses of land, respectively, the subscript $r$ denotes the producing area and $A_{0}$ is the current agricultural land base. Corresponding to each $A_{2 r}$ (nonagricultural land use in region $r$ ), there would also appear in the objective function of the model a cost term, $C_{2 r} A_{2 r}$, where $C_{2 r}$ represents the marginal value product (price) of 1 and in the nonagricultural sector in region $r$. 
This approach permits competition for land between the two sectors, given the price of land in its best alternative use. If the shadow price of land used to grow a particular crop is always below $C_{2 r}$, then this crop will not be produced, and that land will have to be put in crops that can compete with nonagricultural uses at the prevailing price. Thus, price competition for $l$ and can be simulated by varying the price $C_{2 r}$ over some predetermined range in order to analyze the impact of changing land prices on the agricultural sector. Essentially the same approach can be used to analyze changes in the interindustry and interregional distributions of water. In the former case, the approach would be identical, while in the latter, the capital costs of transferring a unit of water between regions would also have to be included in the objective function.

There are essentially two, very much related problems with this method. The first is that, in fact, the demand for land in the nonagricultural sector will itself be indirectly affected by $\mathrm{CO}_{2}$-induced environmental changes in agricultural through the influence of nonmodeled, forward and backward linkages. Whether the indirect effect on the nonagricultural demand for land will be small or large cannot be determined on an a priori basis. If it is negligible, then the treatment of $C_{2 r}$ as exogenous is acceptable. But, even if this assumption is empirically justifiable, a second problem involves selecting the proper price for $C_{2 r^{--}}$one that will reflect the state of regional economic development in all of the producing areas, between 50 and 100 years from today. Since this cannot be done with any degree of precision, a sensitivity analysis of the effect of alternative nonagricultural land prices on the agricultural sector represents the only possible alternative.

The second approach to analyzing the indirect impacts of $\mathrm{CO}_{2}$-induced environmental changes on the distribution of water and land resources involves the use of normative, general equilibrium models of the entire economy. One may recall from Section 7.1.3 that the model developed by Norton and Scandizzo (1981) contains a set of resource constraints

$$
\sum_{i=1}^{n} \sum_{t=1}^{z} d_{i k t} Q_{i t}^{S} \leq b_{k} \quad \text { for a } 11 k
$$


where $d_{i k t}$ represents the amount of input $k$ required to produce a unit of the ith output using technology $t, Q_{i t}^{S}$ is the output of industry $i$ using technology $t$, and $b_{k}$ represents the fixed supply of the $k$ th resource, including water and land. Since the model solves for the optimal values of output, the total amount of the $k$ th resource allocated to industry $i$, or $b_{i k}$, can be quickly calculated from the solution value for $\mathrm{Q}_{\mathrm{it}}^{\mathrm{S}}$ as

$$
b_{i k}=\sum_{t=1}^{z} d_{i k t} Q_{i t}^{S}
$$

These allocations can then be compared to those in the base period to determine the indirect impacts of $\mathrm{CO}_{2}$ on the interindustry distribution of land, water and other primary resources.

The only potentially serious drawback to this approach lies in the fixity of the input-output ratios, $d_{i k t}$. In fact, the resource intensities reflected by these coefficients may themselves be subject to change as a result of $\mathrm{CO}_{2}$ buildup. For example, if increases in $\mathrm{CO}_{2}$ measurably boost yields and increase the water use efficiencies of many crops, other things being equal, one might expect an increase in the average productivities of land and water, although this is by no means certain. (In fact, if the demand for a crop is price elastic, then marginal lands would be brought into production and the average productivity of land used to grow that crop might fall a bit.) It turns out, however, that obtaining new, post $\mathrm{CO}_{2}$ estimates for these coefficients is not as serious a problem as first appears to be the case, provided that one is willing to live with the assumption that only the intensity of agricultural land and water use will be affected by $\mathrm{CO}_{2}$. In that case, the intensity of land (but not water) use per unit of output can be determined in the direct impact phase of the analysis from econometric models. Normative agricultural sector models present something of a problem because they also use fixed resource intensities. However, because of the large number of regions included in the model, the value of the national intensities of water and land resource use will change due to spatial 
adjustments in production within the model. As a result, new average (e.g., national) intensities for both land and water can be obtained from this type of agricultural sector model, and these values can be used to change the

$d_{i k t}$ in the normative, general equilibrium model of the entire economy.

To reiterate, if a normative model of the agricultural sector is used in conjunction with a normative equilibrium model of the macroeconomy, impacts on the interindustry distribution of water and land resources can be estimated as follows:

1. By first solving for the values of agricultural commodity outputs and aggregate resource intensities using a normative model of the agricultural sector;

2. Aggregating these values to a level that is consistent with the $Q_{i t}^{S}$ and the $d_{i k t}$ in the normative macro level; and

3. Solving the macro model, using the values obtained from (1) and (2) as exogenous inputs to the normative, macro model.

This represents the preferred approach to evaluating a broad range of $\mathrm{CO}_{2}$-induced indirect impacts on a comprehensive, rather than piecemeal basis. The best alternative to this approach would involve using a normative agricultural sector model to examine the sensitivity of the distribution of land and water resources to changes in their respective prices and availabilities.

\subsection{EVALUATING CHANGES IN CONSUMER WELFARE}

In Section 3.4 .2 an effort was made to show how $\mathrm{CO}_{2}$-induced environmental changes might have the effect of altering the consumption patterns of consumers through changes in the prices of food and other goods and services which are produced by industries linked either directly or indirectly to the agricultural sector. This section of the report will examine ways of evaluating the impact of these price changes on the welfare of consumers and then show how these changes in consumer welfare can be measured in an applied analytical framework. 


\subsubsection{Measures of Consumer. Welfare}

Under competitive market conditions, changes in producer we lfare can be measured by observable changes in profits (Hotelling 1932). However, in the case of consumers, changes in utility cannot be observed directly, and. therefore surrogate measures must used to capture the impact which changes in prices will have on aggregate consumer welfare. While there are many different measures of this type (Currie et al. 1971), the three that are used most of ten are known as: (1) compensating variation (CV), (2) equivalent variation (EV), and (3) consumer surplus (CS). These three measures of consumer welfare can be defined as follows:

- Compensating Variation--In the event of a welfare loss, CV is the minimum amount of money by which consumers would have to be compensated in order to be as well off after the change as before it. For a welf are gain, CV is the maximum amount of money which can be taken away from consumers, while leaving them just as well off after the change as before it.

- Equivalent Variation--For a welfare loss, EV is the amount of money which consumers would be willing to pay to prevent the change from occurring. In the event of a welfare gain, EV represents the amount of money by which consumers would have to be compensated to forego the change in prices.

- Consumer Surplus--This is measured as the difference between the maximum amount of money consumers would be willing to pay for a good and the amount actually paid.

Both the compensating and equivalent variation can be derived from the standard theory of consumer behavior by asking: what is the minimum cost to consumers of achieving a fixed level of utility, $\bar{U}$, consistent with prices, $P=P_{1}, \ldots, P_{m}$ ? This question can be answered by first considering the following consumer decision problem

$$
\text { Minimize } C=\sum_{i} P_{i} Q_{i}
$$


subject to

$$
U\left(Q_{1}, \ldots, Q_{m}\right)=U
$$

where $Q_{i}$ is the price of the ith good, $P_{i}$ is its price, and $U($ ) represents the utility function of the consumer. The solution to this problem is represented by a system of income compensated demand functions.

$$
Q_{j}=g_{j}(\bar{U}, P) \quad \text { for all } i
$$

Substituting these demand functions back into Equation (7.40) results in a representation of the dual cost function of the consumer

$$
C=C^{\star}(\bar{U}, P)
$$

which indicates the minimum cost to consumers at which a given level of utility can be achieved at fixed prices.

Now consider a change in the prices of final goods and services from $P^{0}$, in the based period, to $P^{1}$ in the subsequent period. In that context, $C V$ represents the difference between the minimum cost of achieving $U^{0}$, at prices, $P^{0}$, and the minimum cost of achieving that same level of utility when prices have changed to $\mathrm{P}^{1}$. Using Equation (7.43), CV can then be represented as

$$
C V=c\left(U^{0}, P^{1}\right)-c\left(U^{0}, P^{0}\right)
$$

By contrast, EV is the difference between the minimum amount of money required to achieve $U^{0}$ at prices $P_{0}$ and the amount of money needed to just achieve $U$ ! when prices are at their original level, $P^{0}$. Thus EV can be represented by 


$$
E V=C\left(U^{1}, P^{1}\right)-C\left(U^{1}, P^{0}\right)
$$

Thus, the only real difference between the two concepts is the reference level . of utility. For CV base period utility, $U^{0}$ is used as a reference, whereas in the case of EV current period utility $U^{1}$ is used as a reference.

$\mathrm{CV}$ and EV are exact measures of changes in consumer welfare, while consumer surplus, with one exception, is an inexact measure. To see why this is true recall that the definition of consumer surplus is the difference between the total willingness of consumers to pay for a given amount of a good and the amount of money they actually pay for it. As such the consumer surplus associated with the consumption of a single good can be expressed mathematically as

$$
\int_{Q_{j}^{0}}^{Q_{j}^{1}} P_{i}\left(Q, M^{0}\right) d Q_{i}-P_{i} Q_{i}
$$

where the first expression in Equation (7.46) represents the total willingness of consumers to pay for $Q_{j}$ at the price, $P_{i}$, when income is constant at $M^{0}$, and the second expression represents the cost of purchasing this good. $\mathrm{CV}$, which is conceptually closer to CS in meaning than EV, can be expressed as

$$
\int_{p_{i}^{0}}^{p_{i}^{1}} g_{j}\left(u^{0}, P\right) d p_{i t}
$$

Note that CS is represented by the area under the indirect demand function for $Q_{j}$ or $P_{j}\left(Q, M_{0}\right)$, while $C V$ is measured by the area under the income compensated demand curve for $Q_{j}$, or $g_{j}\left(U^{0}, P\right)$. The reason why CS is not an exact measure of the amount of compensation, paid or received, that will enable consumers to maintain a constant level of welfare is based on the differences between Equation (7.46) and (7.47). When the price of $Q_{j}$ 
changes, consumers will move along the indirect demand curve until their willingness to pay for an additional unit of that good is equal to the new price, $P_{i}^{1}$. However, as this occurs, the level of utility will also change since it is not specifically constrained in Equation (7.46). In general, then, CS will never equal CV. Nevertheless, there is one condition for which $C S$ is a valid measure of consumer welfare (even though $U$ is not constant). This condition is that changes in utility be independent of changes in income (Chipman and Moore 1976). However, this condition is very restrictive and is not supported by the empirical evidence regarding the structure of consumer preferences (Jorgenson and Lau 1975).

\subsubsection{Estimating Changes in Consumer Welfare}

If consumer surplus is generally not a valid measure of changes in consumer welfare, how can one obtain measures of EV or CV? Once this question has been addressed, it will then be possible to discuss the manner in which various methods for measuring changes in consumer welfare can be implemented in the current research problem.

\section{Exact Measures}

Exact measures of either CV or EV require the use of explicit functional representations of consumer preferences in order to obtain estimates of the parameters of the dual cost function, on which the measurement of $C V$ and EV is based. This can be done using a variety of flexible functional forms (MCFadden 1978) to represent consumer preferences. The two functional representations that are best suited to making statements about changes in consumer welfare are the indirect transcendental logarithmic function and the AIDS dual cost function.

The Transcendental Logarithmic Utility Function (TL). The traditional difficulty associated with obtaining estimates of the parameters of the dual cost function is that both this function, and the income compensated demand curves that are derived from it, contain arguments in utility, which is not directly observable. In order to avoid this problem, economists have until recently been forced to derive estimates for the parameters of the dual cost function using its inverse, the indirect utility function, or 


$$
U=V(P, \bar{M})
$$

The functional form traditionally used to approximate this representation of consumer preferences is known as the indirect transcendental logarithmic utility function (Christensen et al. 1973). The indirect TL utility function is conveniently written as

$$
\ln U=\sum_{i=1}^{m} \alpha_{i} \ln P_{i}^{\star}+\frac{1}{2} \sum_{i=1}^{m} \sum_{j=1}^{m} \alpha_{i j} \ln P_{i}^{\star} \ln P_{j}^{\star}
$$

where $P_{i}^{\star}=P_{j} / M$ are the normalized prices, and $M$ represents total expenditures. Using Roy's (1943) identity

$$
\frac{P_{i} Q_{i}}{M}=-\frac{\partial \ln U}{\partial \ln P_{i}^{*}} / \frac{\partial \ln U}{\partial \ln M} \quad(i=1, \ldots, m)
$$

one obtains a set of budget share demand equations consistent with the indirect utility function

$$
w_{i}=\frac{\alpha_{i}+\sum_{j=1}^{m} \alpha_{i j} P_{j}^{*}}{\sum_{j=1}^{m} \alpha_{j}+\sum_{i=1}^{m} \sum_{j=1}^{m} \alpha_{i j} P_{j}^{\star}} \quad(j=1, \ldots, m)
$$

where $w_{j}$ represents the share of share of consumer expenditure devoted to good $i$, or $w_{i}=P_{i} Q_{j} / M$.

The parameters of the share equations shown in Equation (7.51) can be estimated via econometric methods using readily available time series data on personal consumption expenditures and prices for broad categories of goods. Given specific estimates for the parameters $\alpha_{i}$ and $\alpha_{i j}$, as well as known (or simulated) values for expenditures and prices, the corresponding values of 
$U$ in each period can be calculated directly from the indirect demand function expressed in Equation (7.49). The indirect demand function can then be transformed into an implicit function, the general form of which is

$$
H(U, M, P)=0
$$

in order to obtain the values for $C=M$ that are consistent with the expressions used to calculate $\mathrm{CV}$ and $\mathrm{EV}$.

The AIDS Dual Cost Function. Recently, Deaton and Muellbauer (1980) have identified a class of preferences for which the dual cost function can be manipulated directly in such a way as to yield the traditional Marshallian or uncompensated demand functions. A cost function consistent with this class of preferences can be written

$$
\ln C=\sum_{i=1}^{m} \alpha_{i} \ln P_{i}+\frac{1}{2} \sum_{i=1}^{m} \sum_{j=1}^{m} \alpha_{i j} \ln P_{i} \ln P_{j}+U \prod_{i=1}^{m}\left(\frac{P_{i}}{\beta_{i}}\right)^{\beta_{i}}
$$

Using Shephard's $(1953,1970)$ lemma

$$
w_{i}=\frac{\delta C}{\delta P_{i}}
$$

one can obtain a system of budget share functions

$$
w_{i}=\alpha_{i}+\sum_{j=1}^{m} \alpha_{i j} \ln p_{j}+\beta_{i} \cup \prod_{i=1}^{m}\left(\frac{p_{i}}{\beta_{i}}\right) \beta_{i}
$$

By inverting Equation (7.53), solving for $U$, and then substituting the resulting indirect utility function into Equation (7.54a), the uncompensated AIDS budget share demand equations can be written as 


$$
\begin{aligned}
w_{i}= & \alpha_{i}+\sum_{j=1}^{m} \alpha_{i j} \ln P_{j}+\beta_{i}\left(\ln M-\sum_{i=1}^{m} \alpha_{i} \ln P_{i}\right. \\
& \left.-\frac{1}{2} \sum_{i=1}^{m} \sum_{j=1}^{m} \alpha_{i j} \ln P_{i} \ln P_{j}\right)
\end{aligned}
$$

The parameters of these share equations are estimated from the same data used in the estimation of $\mathrm{TL}$ demands. These parameter estimates can then be substituted back into the AIDS dual cost function, and solutions for $U$ in each time period can be obtained using observed (or simulated) values for actual expenditures and prices. Once Equation (7.53) is properly parameterized in this fashion, it can be evaluated to obtain the numerical values for CV and EV consistent with the calculated values of $U$ and forecast values for prices.

\section{Approximate Measures}

There are basically two different ways of approximating changes in consumer welfare when it is impossible to use explicit functional representations for consumer preferences. The first of these involves the use of formulas which describe the maximum error associated with approximating CV and $E V$ by the area under the indirect demand function (Seade 1978; Willig 1976). If only one price change is being considered, or if the change in several prices can be expressed in terms of the change in a single, consistent price index, Vartia (1976) and Hausman (1981) have shown that no such approximation is necessary. Under these conditions, CV and EV Can be calculated using information obtained from the uncompensated demand functions.

The second approach relies, not on calculations of CV or EV to measure changes in consumer welfare, but on the construction of approximate cost-of-living index numbers. The use of approximate cost-of-living indexes is to be contrasted with the use of exact index numbers, which like CV and EV can be derived from the theory of consumer demand (Diewert 1976). Conceptually, the cost-of-living index is the ratio of the minimum expenditures necessary to achieve a reference level of utility at two sets of prices. Mathematically, this can be expressed as 


$$
p^{r}=\frac{C\left(U^{r}, p^{1}\right)}{C\left(U^{r}, p^{0}\right)}
$$

where $r$ represents the reference period for which utility is held constant. It can readily be seen from Equation (7.55) that if the reference period is taken as occurring before the price change $(r=0)$, then $p^{r}$ is simply the ratio of the two components used to calculate CV. If $r=1$, then Equation (7.55) is related in the same way to EV. Thus, if one can calculate $\mathrm{CV}$ and EV by direct methods, the true cost of living indexes can also be computed.

However, if this is not possible, then these cost-of-living indexes can be approximated by a variety of different formulae (Diewert 1981). The cost-of-living indexes most widely used are the Laspeyres $\left(P_{L}\right)$ and Paasche $\left(P_{p}\right)$ indexes. These two indexes can be defined, mathematically, as

$$
\begin{aligned}
& P_{L}=\frac{\sum_{i=1}^{m} P_{i}^{1} Q_{i}^{0}}{\sum_{i=1}^{m} P_{i}^{0} Q_{i}^{0}} \\
& P_{p}=\frac{\sum_{i=1}^{m} P_{i}^{1} Q_{i}^{1}}{\sum_{i=1}^{m} P_{i}^{0} Q_{i}^{1}}
\end{aligned}
$$

These two indexes differ only insofar as the Laspeyres index is an approximation of the true cost-of-living index using the base period as a reference $(r=0)$, while the Paasche index is consistent with using the current period as a reference from which to measure welfare changes. Thus, $P_{L}$ is conceptually close to $C V$, whereas $P_{P}$ is closely allied in meaning to EV. 
Both $P_{L}$ and $P_{p}$ can be constructed from available, or simulated, price and consumption data. The calculated values for these two index numbers can then be used to make statements about the effect of price changes on consumer welfare based on revealed preference theory (Samuelson 1938). This can be done by comparing the values of these two cost-of-living indexes in each period with a money income index

$$
I_{M}=\frac{\sum_{i=1}^{m} P_{i}^{1} Q_{i}^{1}}{\sum_{i=1}^{m} P_{i}^{0} Q_{i}^{0}}
$$

If $I_{M}>P_{L}$, it can be shown by revealed preference theory that consumers must be better off in the current year than in the base year. On the other hand, if $I_{M}<P_{p}$, then consumers must surely be worse off in the current year than in the base year. Thus, Laspeyres and Paasche cost-of-living indexes can only be used to make relative statements about the direction of consumer welfare changes. Furthermore, those statements that can be made are expressed in terms of a set of sufficient conditions for making welfare comparisons between two periods, as opposed to a set of necessary and sufficient conditions.

\subsubsection{Applied Analysis of a Consumer Welf. Changes}

The actual measurement of changes in consumer welfare associated with the indirect impacts due to increases in the ambient concentration of $\mathrm{CO}_{2} \mathrm{will}_{1}$ depend, first, on whether the models used to estimate these impacts contain explicit demand functions for the final goods and services that are included in the model and, secondly, on the type of demand functions used in the model. If a traditional input-output model is used, then it will not be possible to calculate any measures of the change in the consumer welfare due to $\mathrm{CO}_{2}$ because these models do not provide sufficient information to even calculate Laspeyres and Paasche cost-of-living indexes. Generally speaking, however, normative models, input-output based econometric models and regional econometric models either contain explicit representations for consumer 
demands or else these demand equations are implicit in the reduced form of the model equations. If the representation of final demands is based on a complete system of demand equations, derived from an explicit characterization of consumer preferences, as in the TL or AIDS functional forms, then CV and EV can be calculated exactly, consistent with simulated changes in prices. Such a feature lies within the current capability of normative general equilibrium, input-output based econometric models, and some multi-regional econometric models.

If the demand functions in the model are not derived systematically from an explicit representation of consumer preferences, then changes in consumer we If are can be measured in one of three ways:

1. directly (but erroneously), by the calculation of consumer surplus;

2. indirectly by using an appropriate error formulation to convert consumer surplus into either a compensating or equivalent variation; or

3. by using the Laspeyres and Paasche cost-of-living index numbers to examine relative changes in welfare.

Finally, if the model does not contain either explicit or implicit final demand schedules within its structure, but does produce estimates of consumption and prices, then cost-of-living indexes can be employed to make welf are comparisons between two periods, using consumption and price information provided by the model. This can be done within the framework of multi-regional econometric models.

As important as the theoretical and technical issues associated with developing estimates of the impact of increases in the ambient concentration of $\mathrm{CO}_{2}$ on consumer welfare may be, any quantitive estimates that are obtained must be viewed with a certain amount of skepticism. For whether consumer welfare changes are measured correctly in theory or not, it still remains that the parameters of the demand and/or dual cost function on which these measures depend are estimated on the basis of historical data. As in the case of all econometrically estimated models, these parameters reflect a mixture of current and past preferences, not those which will prevail 50 or 
100 years from today. During that time, substantial changes in consumer preferences can be expected to occur which, in turn, will unquestionably lead to marked changes in consumer purchasing behavior and expenditure patterns. Predicting the future structure of consumer preferences, while it may be of great importance, is not a task that the discipline of economics can perform.

\subsection{MODEL SUMMARY}

The ideal approach for assessing the indirect impacts of $\mathrm{CO}_{2}$-induced environmental changes in the agricultural sector on other sectors of the economy would involve embedding a highly disaggregated model of the U.S. agricultural sector within the structure of a price-endogenous, general equilibrium model of the U.S. economy. In such a model, not only the demands for primary resources but also the demands for intermediate goods would be functionally related to industry output and input prices. The final demand for each industry's output would be treated as a function of consumer income and prices through a system of flexible demand functions. Thus, the equilibrium prices and output levels in primary resource markets and commodity markets would be determined simultaneously.

The requirements described briefly above constitute the major elements of a disaggregated, general equilibrium model of the economy. Models that are theoretically fairly faithful to the principles of general equilibrium in many markets, simultaneously, do exist (Hudson and Jorgenson 1974; Jorgenson and Frammeni 1980). However, they are very small models, with no more than six, highly aggregated producing sectors in the entire economy. But, while small in terms of the number of sectors included, the number of equations required to duplicate a general equilibrium in these models is quite large. A model containing 10 to 15 agricultural commodities and a hundred or more producing sectors would be very, very large indeed by current economic model standards, if primary resource, intermediate and final demands were all treated in the manner suggested above.

This report did not explore building such a model, although a small model of this type has been proposed for use in analyzing with economic impacts of $\mathrm{CO}_{2}$ buildup (Smith 1980). Instead, previous sections of this chapter have 
focused on using information that is obtained from a disaggregated multi-regional agricultural sector model and then plugging this information into different types of macro-models of the U.S. economy. This represents a "linked model" approach, as opposed to an "embedded model" approach. The decision to proceed in this direction was based on two general considerations. The first is that multi-regional agricultural sector models are much better structured to simulate the economic impact of $\mathrm{CO}_{2}$-induced environmental changes on agricultural production than an embedded national model of the agricultural economy. Since the former kind of model is regionally disaggregated, not only can it accept region-specific climate or yield scenarios, but it can also show how $\mathrm{CO}_{2}$ buildup is likely to affect the current geographic distribution of production for many different commodities. On the other hand, an embedded national model of the agricultural sector could only accept national yield or climate inputs. Consequently, any potential impacts associated with the spatial redistribution of agricultural activity would be masked in national aggregates, and even these results would undoubtedly be misleading due to the aggregation problem.

The second reason for not exploring the embedded model concept in further detail is due to the large amount of uncertainty that clouds our current understanding of just how $\mathrm{CO}_{2}$ buildup will affect both the environment and the economy. It seems fair to say that increases in the atmospheric concentration of $\mathrm{CO}_{2}$ probably will not become a potential problem for at least another 25 or 30 years, and probably longer. Consequently, either the potential costs or benefits foregone due to waiting to build new and relatively expensive models will not be as great as in a situation where fairly catastrophic impacts were expected to occur with a high degree of confidence in a decade or so. As a result, it can be argued that a more efficient allocation of resources to economic impact analysis in the case of $\mathrm{CO}_{2}$ would involve first exploring the potential effects of $\mathrm{CO}_{2}$ buildup using existing models. If this type of analysis shows that the economy will be particularly sensitive to small changes in $\mathrm{CO}_{2}$ concentrations, more sophisticated approaches can be explored, developed and implemented at that time. 
Given the decision to explore the potential for linking multi-regional agricultural sector models with macro-models of the U.S. economy, four different types of models were examined. They included:

- input-output models

- input-output models based econometric models

- normative general equilibrium models

- multi-regional econometric models

A brief discussion of the primary advantages and disadvantages of using each of these types of models to analyze the indirect economic impacts due to $\mathrm{CO}_{2}$-induced environmental changes in the agricultural sector follows. This information is sumarized in terms of the structural features of each type of mode 1 in Table 7.3 .

\subsubsection{Input-Output Models}

In all, four different forms of input-output techniques were examined. These consisted of:

1. The traditional Leontief-type model which has been used largely to explore the impact of changes in final demands on industry and economy-wide gross output.

2. The resource-requirements approach to input-output modeling which has been used to examine the impact of changes in final demands on the availability of natural resources.

3. Input-output, optimization models which have been used to analyze the impact of resource availability on value added and the shadow prices of these resources for alternative levels of final demand.

4. Supply-constrained input-output models that have been used, largely in Eastern European countries, to examine the impacts of resource shortages and industrial bottlenecks on gross output.

The primary advantage of all of these methods lies in the great detail they contain about interindustry transactions, and the systematic way in which various types of multipliers can be used to examine impacts associated with changes in final demands or resource supplies. Unfortunately, the general 


\section{TABLE 7.3. Summary of Structural Features of Models for Determining the Indirect Impacts of $\mathrm{CO}_{2}$-Induced Environmental Changes}

\begin{tabular}{|c|c|}
\hline Structural Feature & Input-Output \\
\hline $\begin{array}{l}\text { Determination of Final } \\
\text { Demands }\end{array}$ & Exogenous to model \\
\hline $\begin{array}{l}\text { Determination of Inter- } \\
\text { industry Product Flows }\end{array}$ & $\begin{array}{l}\text { By inverting the matrix } \\
\text { of interindustry technical } \\
\text { coefficients }\end{array}$ \\
\hline
\end{tabular}

Determination of Primary Resource Use by Industry

Best accomplished by using supply constrainted inputoutput models
Method for Interfacing

with Agricultural
Sector Models

Determination of Changes in Consumer Welf are

Determination of Ind irect Impacts on the Interindustry Allocation of Land and Water
By changing final demands for agricultural

No valid measures for changes in consumer we if are can be derived from input-output mode's

Methods include: (a) addition of water and land content matrices to the basic mode l, (b) supply constrained models, (c) and models
Econometric Input-Output Determined simultaneousiy using a system of consumer demand equations

Intermediate product demands are based on fixed input-

output coefficients contained in the interindustry transaction matrix

Primary input demands for capital, labor, and energy are determined simultaneously using a system of nput demand functions

By changing agricultural product prices and constraining re levant gricultural agricultural products

Changes in consumer surplus and compensating variation can be evaluated from the system inal demand equations

Primary resource coverage of existing models does not include $l$ and and water
Normative General Equilibrium Similar to econometric-inputoutput structure

Similar to econometric input-output structur

Multi-Regional Regional output by industry determined endogenous $1 y$

Inter industry product flows are not modeled within regions

Primary inputs are allocated according to their value in use, consistent with fixed input-output coefficients

Similar to methods used in conjunction with econometric

imilar to methods used in conjunction with econometric input-autput mode1s

$L$ and and water balance equations can be included within the model
By constraining gross output, by industry, at the

Cost-of-living index numbers and in some cases changes in culated from model outputs

Primary resource coverage water
Employment, investment and capital stocks by industry are determined endogenously does not include land and 
equilibrium that is duplicated by the structure of input-output models is extremely unrealistic for the analysis of long-run economic problems. In particular, prices play no role in determining industry output and final demands are treated as exogenous. As such, these types of methods are not at all appropriate for examining the indirect impacts of $\mathrm{CO}_{2}$-induced environmental changes in the agricultural sector.

\subsubsection{Input-Output Based Econometric Models}

These types of models retain the interindustry detail present in the traditional input-output models, with no modification to the structure of interindustry demands for intermediate goods. On the other hand, final demands in these models are price and income dependent while the demands for primary resources are functionally related to industry output and resource prices. These models also have an advantage over traditional input-output models in that they are dynamic, in time, due to the use of stock adjustment mechanisms to determine capital investment. Thus, these types of models more closely approach the ideal outlined at the beginning of this section than do the input-output models from which they are derived.

Perhaps the most attractive feature of these models is that the methods for employing them in conjunction with linked agricuitural sector models have al ready been developed. As such, projecting the economy-wide impacts due to $\mathrm{CO}_{2}$-induced price and output changes in agricultural commodity production would not be a technically difficult task. An important limitation of input-output based econometric models is that primary resources do not generally include water and land, both of which may be impacted substantially by $\mathrm{CO}_{2}$ buildup. This can be remedied, either by embedding resource content matrices for land and water within the model, as shown in Section 7.2.1, or by estimating new input demand functions for these inputs similar to those for other primary resources.

\subsubsection{Normative General Equilibrium Mode ls}

These models are appealing, not only because they provide competitive general equilibrium solutions that are consistent with an optimal response to $\mathrm{CO}_{2}$-induced price and output changes within the agricultural sector, but 
also because they contain features which make it relatively easy to examine the impact of these changes on the interindustry distribution, and the prices of water and land resources. In addition, these models can be designed to incorporate a very large interindustry transaction matrix within the constraint set, allowing them to provide the same, or even a greater level of sectoral detail than is present in input-output based econometric models. Furthermore, simulating the impacts of $\mathrm{CO}_{2}$-induced economic changes in the agricultural sector on other sectors of the economy can be accomplished by essentially the same methods used to link an agricultural model to an input-output based econometric model.

The major drawback of this approach is that no such large model of the U.S. economy currently exists. This probably reflects the fact that most business and policy-related questions which are addressed in the framework of other forms of macroeconomic models do not require normative answers. In the case of $\mathrm{CO}_{2}$, however, a normative framework would be invaluable since all of the adjustments that will be made to $\mathrm{CO}_{2}$ buildup can best be viewed in a long-run setting, free of institutional and technical rigidities.

\subsubsection{Multi-Regional Econometric Models}

The advantage of using these models is that they make it possible to articulate the indirect economic impacts associated with $\mathrm{CO}_{2}$ on a regional basis. However, these models are usually driven by national values for industry output, or by some other measure of economic activity which reflects the importance of national demands for regionally produced goods and services. Thus, the regional impacts of $\mathrm{CO}_{2}$ can only be determined after the indirect impacts of $\mathrm{CO}_{2}$ buildup on the national economy have been determined by means of an input-output based econometric model or a normative general equilibrium model of the U.S. Consequently, multi-regional econometric models should not be viewed as a substitute for economy-wide models. Rather, the ir advantage lies in a complementary role.

Adapting these models for use in the current research context would not require any additional model development, since these models are indifferent to the nature of the forces which change the values of the national variables 
that drive them. The only potentially serious problem associated with their use is that because these models also determine regional agricultural output, it would be surprising if there were not discrepancies between the levels of regional agricultural activity projected by one of these models and the levels projected by the agricultural sector mode1. Large differences would create a particularly serious problem of interpretation in regions where national demands for agricultural commodities constitutes a dominant force in the local economy. In order to avoid these types of problem, it would also be possible to short circuit the effect of national agricultural demands by holding regional agricultural output constant at levels that are consistent with agricultural sector model projections and simulating the resulting impacts on other sectors of the regional economy.

Finally, in the event that a national, rather than a multi-regional agricultural sector model, is used to conduct the direct impact analysis phase of the research, it would be possible to use a multi-regional econometric model in conjunction with a macro-model of the national economy to examine both the direct and indirect impacts of $\mathrm{CO}_{2}$ buildup at the regional level.

\subsubsection{Recommendations}

Table 7.4 summarizes the selected structural features of the models that appear to be best suited for evaluating the indirect impacts of $\mathrm{CO}_{2}$-induced environmental impacts which originate in the agricultural sector.

At this time, it seems clear that input-output based econometric models represent the best variable alternative for examining the indirect impacts associated with $\mathrm{CO}_{2}$-induced environmental changes in the agricultural sector. The Battelle Memorial Institute's FORSYS model has already been used in a methodologically similar exercise to analyze the impact of selected agricultural policies on general price inflation in the economy and could easily be adapted for use in the current research context. Since the final demands in FORSYS are derived from the indirect transcendental logarithmic utility function, calculation of exact measures of consumer welfare changes due to $\mathrm{CO}_{2}$-induced price changes would also be possible. However, to use this, or any other similar model to examine possible changes in the 
TABLE 7.4. Summary of Selected Structural Features of FORSYS, MASTER and the Proposed Normative General Equilibrium Model (NGE), Including Recommended Changes to FORSYS

\begin{tabular}{|c|c|c|}
\hline \multirow[b]{2}{*}{ Model Characteristic } & \multicolumn{2}{|l|}{ FORSYS } \\
\hline & Structural Features & Changes \\
\hline Fina 1 Demands & $\begin{array}{l}\text { Two-Stage Demand System: } 9 \\
\text { aggregate and } 54 \text { disaggreg- } \\
\text { ated consumption sectors }\end{array}$ & None \\
\hline Production Sectors & $\begin{array}{l}107 \text { sectors, } 5 \text { of which } \\
\text { represent agricultura } 1 \\
\text { production }\end{array}$ & None \\
\hline Primary Resource Sectors & $\begin{array}{ll}\text { Capital } & -100 \text { investment demand } \\
& \text { and } 22 \text { capital supplying } \\
& \text { sectors } \\
\text { Labor } & -107 \text { labor demand sectors } \\
\text { Energy } & \text { residential plus } 107 \\
& \text { industry demand sectors } \\
& \text { and } 3 \text { supply sectors }\end{array}$ & $\begin{array}{l}\text { Expand primary } \\
\text { resource detail } \\
\text { to include both } \\
\text { supply and demand } \\
\text { sectors for land } \\
\text { and water }\end{array}$ \\
\hline Model Outputs & $\begin{array}{l}\text { Consumption and prices for final } \\
\text { goods } \\
\text { GNP and aggregate price level } \\
\text { Sectoral output and price indexes } \\
\text { Sectoral investment and capital } \\
\text { stock } \\
\text { Sectoral employment, productivity } \\
\text { and wages } \\
\text { Unemployment } \\
\text { Personal and disposable income } \\
\text { Sectoral ener ay consumption }\end{array}$ & $\begin{array}{l}\text { Interindustry } \\
\text { allocation of land } \\
\text { and water }\end{array}$ \\
\hline
\end{tabular}

\begin{tabular}{l} 
NGE \\
\hline Structural Features \\
Two-Stage Demand System: \\
level of disaggregation not \\
yet determined \\
Determination of sectors \\
would be based on BEA or \\
BLS input-output tables \\
Primary resources can include \\
land, labor, cap ital, and \\
water; total resource \\
supplies can be fixed or \\
determined endogenously
\end{tabular}

Consumption and prices for final goods

GNP

Sectoral output and shadow prices

Interindustry allocation of

primary resour
shadow prices
MASTER

Structural Features

Final demands are.not modeled explicitly; total demand for sectoral output can be approx mated by sectoral shipments.

Current model has 11 sectors; disaggregation to 2-digit SIC level planned for FY1983

Capital - investment and capital stock in residentia and commercial con-
struction and manufacstruction and manufac-
turing.

Labor - employment and wages including import for

Energy - Consumption by residential and 11 production sectors

Sectoral output

Sectoral investment

Sectoral employment and wages Unemp loyment

Personal and disposable income

Sectoral energy consumption

Net migration

Natural population change

Population 
interindustry distribution of water and land resources would require some additional research and modification to basic model structure.

The potential of normative general equilibrium models in the analysis of indirect impacts appears to be great. In concept, these models can fulfill all of the demands placed on input-output based econometric models, while at the same time permitting a more detailed analys is of the impact of $\mathrm{CO}_{2}$-induced environmental changes in the agricultural sector on the equilibrium distribution and prices of water and land resources. More importantly, the normative solutions provided by such a model would make it possible to evaluate the full capacity of the economy to respond to $\mathrm{CO}_{2}$ buildup, whereas the results obtained from an input-output based econometric model would inevitably reflect existing institutional and technological rigidities within the economy. In that general context, further investigation of the feasibility and costs of constructing a fairly detailed normative general equilibrium model of the U.S. economy is also recommended.

Finally, multi-regional models can be employed to explore the indirect impacts associated with $\mathrm{CO}_{2}$ buildup at a fairly minute level of geographic detail. No additional development methods would be required to conduct this type of analysis. Of the models examined, the PNL MASTER model and the MRMI model appear to be the two best candidates for investigations that will focus on the regional economic impacts associated with $\mathrm{CO}_{2}$-induced environmental changes in the agricultural sector. This type of analysis would be particularly important in examining the impact of extreme climatic changes on rural regions whose local economies might be highly vulnerable to even fairly small declines in agricultural output or fluctuations in agricultural prices. 


\section{REFERENCES}

Augustinovics, M. 1970. "Methods of International and Intertemporal Comparisons of Structure." In Contributions to Input-0utput Analys is, Vol. 1, eds. A. P. Carter and A. A. Brody, pp. 249-269. North Holland, Amsterdam, The Nether lands.

Aukrust, 0. 1970. "RIMP-1: A Model of Price and Income Distribution Mechanism of an Open Economy." Review of Income and Wealth 16(1):148-162.

Ballard, K. P. and R. 0. Gustley. 1980. "NRIES: A Bottom-up Multiregion Model of the U.S. Economy." Paper presented at a Conference on an Assessment of the State-of-the-Art in Regional Modeling at MIT-Harvard Joint Center for Urban Studies, April 23-25, 1980, Cambridge, Massachusetts.

BEA. 1974. The Input-0utput Structure of the U.S. Economy 1967. Vol. 3, pp. 24-56. U.S. Department of Commerce, Government Printing Office, Washington, D.C.

Belzer, D. B. and J. M. Roop. 1981. "Technological Change, Prices and the Demand for Energy in U.S. Manufacturing." Paper presented for the Symposium on Technological Change and Productivity, December 2, Washington, D.C.

Bullard, C. W. and R. A. Herendeen. 1975a. "The Energy Cost of Goods and Services." Energy Policy 3(4):268-275.

Bullard, C. W. and R. A. Herendeen. 1975b. "Energy Impact of Consumption Dec is ions." Proceedings of the IEEE, March 1975, pp. 484-493.

Bureau of Economic Analys is (BEA). 1979. The Detailed Input-Output Structure of the U.S. Economy: 1972. U.S. Department of Commerce, U.S. Government Printing office, Washington, D.C.

Chipman, J. S. and J. C. Moore. 1976. "The Scope of Consumer's Surplus Arguments." In Evolution, Welfare and Time in Economics: Essays in Honor of Nicholas Georgescu-Rogen, eds. A. M. Tang et al., pp. 69-123. Lexington Books, D. C. Heath and Co., Lexington, Massachusetts.

Christensen, L. R. et al. 1973. "Transcendental Logarithmic Production Frontiers." Review of Economics and Statistics 55(1):28-45.

Currie, J. M. et al. 1971. "The Concept of Economic Surplus and its Use in Economic Analysis. The Economic Journal 81(324):741-799. Deaton, A. and J. Muellbauer. 1980. "An Almost Ideal Demand [AIDS] System."
American Economic Review 70(3):312-326. 
Diewert, W. E. 1971. "An Application of the Shephard Duality Theorem: A General-

ized Leontief Production Function." Journal of Political Economy

$79(3): 481-505$.

Diewert, W. E. 1976. "Exact and Superlative Index Numbers." Journal of Econometrics 4(1):115-145.

Diewert, W. E. 1981. "The Economic Theory of Index Numbers: A Survey." In Essays in the Theory and Measurement of Consumer Behavior, in Honor of Sir Richard Stone, ed. A. Deaton, pp. 163-208. Cambridge University Press, London, England.

Frank, M. D. and B. R. Beattie. 1979. The Economic Value of Irrigation Water in the Western United States: An Application of Ridge Regression.

Technical Report No. 99, Texas Water Resources Institute, Texas A\&M University, College Station, Texas.

From, D. et al. 1980. "The Wharton EFA Multiregional, Econometric Model: A Bottom-Up, Top-Down Approach to Constructing a Regionalized Model of the U.S. Economy." In Modeling the Multiregional Economic System, eds. F. G. Adams and N. J. Glickman, pp. 89-106. Lexington Books, D. C. Heath and Co., Lexington, Massachusetts.

Fuss, M. et al. 1978. "A Survey of Functional Forms in the Economic Analysis of Production." In Production Economics: A Dual Approach to Theory and Applications, eds. M. Fuss and D. McFadden, pp. 219-268. North Holland, Amsterdam, The Netherlands.

Gardner, B. D. and H. H. Fullerton. 1968. "Transfer Restrictions and the Misallocation of Irrigation Water." American Journal of Agricultural Economics 50(4):556-571.

Ghosh, A. 1958. "Input-Output Approach to an Allocative System." Economica $25(1): 58-64$.

Giarratani, F. 1976. "Application of an Interindustry Supply Model to Energy Issues." Environment and Planning 6(4):447-454.

Giarratani, F. 1981. "A Supply-Constrained Interindustry Model: Forecasting Performance and Evaluation." In Regional Development Under Stagnation, eds. W. Buhr and P. Friedrick, pp. 281-291. Nomos Verlags-Gesellschaft, Baden-Baden, West Germany.

Glickman, J. J. 1974. "Son of 'The Specification of Regional Econometric Models." Papers of the Regional Science Association 32(1):155-177.

Gorman, W. M. 1953. "Community Preference Fields." Econometrica 21(1):63-80. 
Harris, C. C. 1980. "The Development and Use of the Multiregional, MultiIndustry Forecasting. Mode 1." Paper presented at a Conference on an Assessment of the State-of-the-Art in Regional Modeling, MIT-Harvard Joint Center for Urban Studies, April 23-25, 1980, Cambridge, Massachusetts.

Hausman, J. A. 1981. "Exact Consumer's Surplus and Deadweight Loss." American Economic Review 71(4):662-676.

Herendeen, R. A. 1973. The Energy Cost of Goods and Services. ORNL/NSF/ EP-58, Oak Ridge National Laboratory, Oak Ridge, Tennessee.

Hotelling, H. 1932. "Edgeworth's Taxation Paradox and the Nature of Demand and Supply Functions." Journal Political Economy 40(5):577-616.

Hudson, E. A. and D. W. Jorgenson. 1974. "U.S. Energy Policy and Economic Growth." Bell Journal of Economics and Management Service 5(2):461-514.

Isard, W. and R. E. Kuenne. 1953. "The Impact of Steel Upon the Greater New York-Philadephia Industrial Region." Review of Economics and Statistics $35(4): 289-301$.

Jorgenson, D. W. and B. Fraumeni. 1980. Substitution and Technical Change in Production. Harvard Institute of Economic Research Discussion Paper No. 752, Harvard University, Cambridge, Massachusetts.

Jorgenson, D. W. and L. J. Lau. 1975. "The Structure of Consumer Preferences." Annals of Economic and Social Measurement 4(1):49-101.

Kelso, M. M. et al. 1973. Water Supplies and Economic Growth in an Arid Environment: An Arizona Case Study. University of Arizona Press, Tucson, Arizona.

Kite, R. C. and J. M. Roop. 1981. "Changing Agricultural Prices and The ir Impact on Food Prices Under Inflation." American Journal of Agricultural Economics 63(5)956-961.

Klein, L. R. 1969. "The Specification of Regional Econometric Models." Papers of the Regional Science Association 23(1):105-115.

Klein, L. R. and N. J. Glickman. 1977. "Econometric Model-Building at the Regional Level." Regional Science and Urban Economics 7(1):3-23.

Kutscher, R. and C. Bowman. 1974. "Industrial Use of Petroleum: Effect on Employment." Monthly Labor Review 97(1):3-8.

Lewis, C. W. et al. 1973. Regional Growth and Water Resource Investment. Lexington Books, D. C. Heath and Co., Lexington, Massachusetts.

Leont ief, W. W. 1951. The Structure of the American Economy. 0xford University Press, Fair Lawn, New Jersey. 
Lofting, E. M. and H. C. Davis. 1968. "The Inter industry Water Content Matrix: Applications on a Multiregional Basis." Water Resources Research $4(4): 689-695$.

Lofting, E. M. and P. H. McGauhey. 1963. Economic Evaluation of Water, Part III. Contribution No. 67, Water Resources Center, University of California, Berkeley, California.

Lofting, E. M. and P. H. McGauhey. 1968. Economic Evaluation of Water, Part IV. Contribution No. 116, Water Resources Center, University of California, Berkeley, California.

Manne, A. S. et a1. 1978. "Computation of Competitive Equilibrium by a Sequence of Linear Programs." Department of Operations Research, Stanford University, Palo Alto, California.

Miernyk, W. H. 1969. "An Interindustry Forecasting Model with Water Quantity and Quality Constraints." In Systems Analys is for Great Lakes Water Resources, Proceedings of the Fourth Symposium on Water Research of the Ohio State University, pp. 49-58. Water Resources Center, Ohio State University, Columbus, Ohio.

Moore, F. T. and J.W. Petersen. 1955. "Regional Analysis: An Inter-Industry Model of Utah." Review of Economic and Statistics 37(3):376-377.

Muellbauer, J. 1975. "Aggregation, Income Distribution and Consumer Demand." Review of Economic Studies 62(2):525-543.

Mue 11bauer, J. 1976. "Commun ity Preferences and the Representative Consumer." Econometrica 44(4):979-999.

Murtagh, B. A. and M. A. Sanders. 1978. "Large-Scale Linearly Constrained Optimization." Mathematical Programming 14(1):41-72.

Norton, R. D. and Scandizzo. 1981. "Market Equilibrium Computations in Activity Analys is Models." Operations Research 29(2):243-262.

01sen, R. J. et al. 1977. MULTIREGION: A Simulation-Forecasting Model of BEA Economic Area Population and Employment. ORNL/RUS-25, 0ak Ridge National Laboratory, Oak Ridge, Tennessee.

01sen, R. J. et al. 1980. "MULTIREGION: An Experiment in Regional and Interregional Analysis." Paper presented at a Conference on an Assessment of the State-of-the-Art in Regional Modeling at MIT-Harvard Joint Center for Urban Studies, Apri1 23-25, 1980, Cambridge, Massachusetts.

Plessner, Y. 1957. "Activity Analysis, Quadratic Programming and General Equilibrium." International Economic Review 8(1):168-179. 
Pred, A. 1966. The Spatial Dynamics of U.S. Industrial Growth 1800-1914. MIT Press, Cambridge, Massachusetts.

Richardson, H. W. 1973. Regional Growth Theory. MacMillan, New York, New York.

Roy, R. 1943. De L'Utilitie: Contribution a la Theorie des Choix. Hermann et Cie, Pur is, France.

Sarmue lson, P. A. 1938. "A Note on the Pure Theory of Consumer Behavior." Economica 5(1):61-71.

Seade, J. 1978. "Consumer's Surplus and Linearity of Engle Curves." Economic Journal 88(4):411-523.

Shephard, R. W. 1953. Cost and Production Functions. Princeton University Press, Princeton, New Jersey.

Shephard, R. W. 1970. Theory of Cost and Production Functions. Princeton University Press, Princeton, New Jersey.

Smith, V. L. 1980. Research Proposal Submitted to the Department of Energy for Consideration by the Carbon Dioxide Effects Research and Assessment Program of the Office of Health and Environmental Research. Department of Economics, University of North Carolina, Chapel Hill, North Carolina.

Tijoriwala, A. G. et al. 1968. The Structure of the Arizona Economy: Output Interrelationships and The ir Effects on Water and Labor Requirements: Part 1. Arizona Agricultural Experiment Station Technical Bulletin No. 180, University of Arizona, Tuscon, Arizona.

Vartia, Y. 0. 1976. Relative Changes and Index Numbers. Research Institute of the Finn ish Economy, Helsinki, Finland.

Willig, R. 1976. "Consumer's Surplus Without Apology." American Economic Review 66(4):589-597.

Wright, D. J. 1975. "The Natural Resource Requirements of Commodities." Applied Economics 5(1):31-39.

Yaron, D. 1967. "Incorporation of Income Effects into Mathematical Programming Models." Metroeconometrica 19(1):141-160. 
• 


\section{DISTRIBUTION}

No. of

Copies

OFFSITE

50 M. Riches

Carbon Dioxide Research Division

U.S. Department of Energy

A1-3101 Century 21 B 1dg.

Germantown, MD 20545

27 DOE Technical Information Center

D. Baker

Crop Simulation Unit

Agricultural Research Service

U.S. Department of Agriculture

Mississippi State, MS 39762

G. Collins

Department of Agricultural Economics

Texas A\&M University

College Station, TX 77843

J. Edwards

Department of Agricultural and Re source Economics

Oregon State University

Corvallis, OR 97330

B. English

Center for Agricultural and Rural Development

Iowa State University

Ames, IA 50010

A. Hecht

Director, National Climate Program

National Atmospheric and

Oceanographic Administration

Room 508, Bldg. 5

6010 Executive Blvd.

Rockville, MD 20852
No. of

Copies

OFFSITE

J. Hoffman

Energy Policy Division

Environmental Protection Agency

Room 431 West Tower, Waterside Mall

401 M St. S.W.

Washington D.C. 20460

C. Howe

Department of Economics

University of Colorado

Bou lder, CO 80309

N. Strommen

World Agricultural Outlook Board

U.S. Department of Agriculture

South Agriculture Bldg.

Independence Ave, SW 20250

R. Taylor

Department of Agricultural Economics

Montana State University

Bozeman, MT 59715

T. Waltz

National Climate Program

National Atmospheric and

Oceanographic Administration

Room 508, B 1dg. 5

6010 Executive Blvd.

Rockville, MD 20852

15 M. White

No. 1 Cyclotron Rd.

Lawrence Berke ley Laboratory

Berkeley, CA 94720 
No. of

Copies

ONSITE

DOE Richland Operations Office

H. E. Ransom

61 Pacific Northwest Laboratory

J. M. Callaway

F. J. Cronin

J. W. Currie (50)

D. E. Deonigi

J. Tawil

Technical Information (5)

Publishing Coordination (2) 